Supporting Information for

\title{
Fluorescent Molecular Cages with Sucrose and Cyclotriveratrylene Units for the Selective Recognition of Choline and Acetylcholine
}

Łukasz Szyszka, * Marcin Górecki, Piotr Cmoch, and Sławomir Jarosz* Institute of Organic Chemistry, Polish Academy of Sciences, Kasprzaka 44/52, 01-224 Warsaw, Poland 


\section{Table of Contents}

Table S1. Comparison of ${ }^{1} \mathrm{H}$ and ${ }^{13} \mathrm{C}$ NMR signals of $\boldsymbol{P}-\mathbf{5} \mathbf{a}, \boldsymbol{P}-\mathbf{5 b} \mathbf{b}, \boldsymbol{M}-\mathbf{5} \mathbf{a}$, and $\boldsymbol{M}-\mathbf{5 b}$. .......................... 3

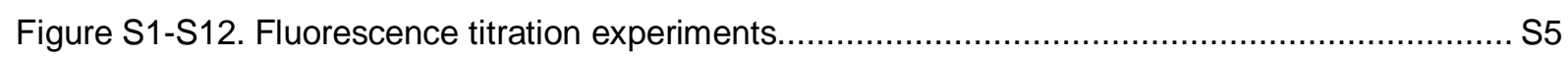

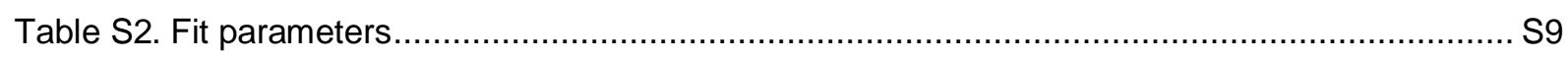

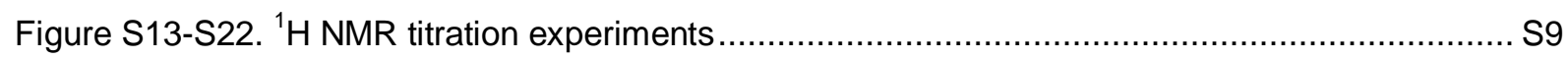

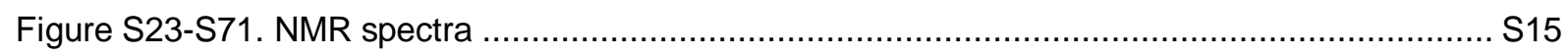

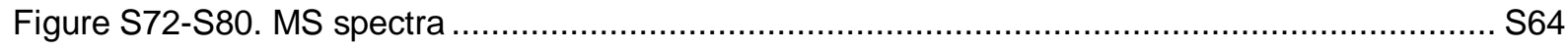

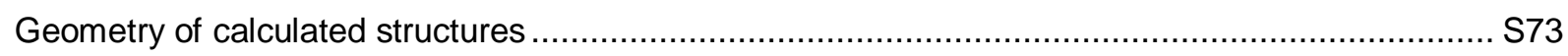


Table S1. Comparison of ${ }^{1} \mathrm{H}$ and ${ }^{13} \mathrm{C}$ NMR signals of $\boldsymbol{P}-5 \mathrm{a}, \boldsymbol{P}-\mathbf{5} \mathbf{b}, \boldsymbol{M}-\mathbf{5}$, and $\boldsymbol{M}-\mathbf{5} \mathbf{b}$.

\begin{tabular}{|c|c|c|c|c|}
\hline & & $d^{13}$ C NMR & ical shifts $\delta(p p I$ & \\
\hline Glucose part & $P-5 a$ & $P-5 b$ & $M-5 a$ & $M-5 b$ \\
\hline $\mathrm{H}-1 / \mathrm{C}-1$ & $5.19 / 90.7$ & $5.55 / 88.7$ & $5.60 / 91.4$ & $5.54 / 88.9$ \\
\hline $\mathrm{H}-2 / \mathrm{C}-2$ & $3.30 / 79.9$ & $3.61 / 79.3$ & 3.38/79.7 & $3.64 / 79.5$ \\
\hline $\mathrm{H}-3 / \mathrm{C}-3$ & $3.79 / 81.6$ & $3.92 / 82.3$ & $3.72 / 81.4$ & $3.93 / 82.4$ \\
\hline $\mathrm{H}-4 / \mathrm{C}-4$ & $3.56 / 77.5$ & $3.57 / 78.0$ & $3.41 / 77.7$ & $3.70 / 77.9$ \\
\hline $\mathrm{H}-5 / \mathrm{C}-5$ & $3.81 / 70.6$ & $4.13 / 69.9$ & $3.90 / 70.4$ & $4.19 / 69.4$ \\
\hline $\mathrm{H}-6 \mathrm{a}+\mathrm{H}-6 \mathrm{~b} / \mathrm{C}-6$ & $3.17+3.53 / 68.1$ & $3.26+3.31 / 67.6$ & $3.06+3.39 / 67.9$ & $3.20+3.46 / 65.9$ \\
\hline Fructose part & & & & \\
\hline $\mathrm{H}-1^{\prime} \mathrm{a}+\mathrm{H}-1^{\prime} \mathrm{b} / \mathrm{C}-1^{\prime}$ & $3.45+3.65 / 69.9$ & $3.58+3.71 / 74.8$ & $3.66+3.72 / 69.1$ & $3.43+3.80 / 74.8$ \\
\hline C-2' & 104.1 & 104.0 & 104.6 & 103.0 \\
\hline H-3'/C-3' & $4.38 / 84.1$ & $4.24 / 82.5$ & $4.35 / 84.9$ & $4.29 / 83.4$ \\
\hline H-4'/C-4' & $3.83 / 82.4$ & $3.53 / 81.3$ & $4.03 / 83.1$ & $3.06 / 81.8$ \\
\hline $\mathrm{H}-5^{\prime} / \mathrm{C}-5^{\prime}$ & $4.07 / 80.9$ & $3.32 / 79.2$ & $3.92 / 80.8$ & $3.76 / 77.6$ \\
\hline $\mathrm{H}-6^{\prime} \mathrm{a}+\mathrm{H}-6^{\prime} \mathrm{b} / \mathrm{C}-6^{\prime}$ & $3.36+3.54 / 73.0$ & $2.84+3.37 / 70.9$ & $3.46+3.49 / 73.1$ & $2.25+2.95 / 72.7$ \\
\hline Naphthalene linkers & & & & \\
\hline $\mathrm{H}-7 \mathrm{a}+\mathrm{H}-7 \mathrm{~b} / \mathrm{C}-7$ & $4.15+4.45 / 73.1$ & $4.23+4.78 / 73.0$ & $4.04+4.64 / 73.4$ & $4.23+4.94 / 72.8$ \\
\hline $\mathrm{H}-7^{\prime} \mathrm{a}+\mathrm{H}-7^{\prime} \mathrm{b} / \mathrm{C}-7^{\prime}$ & $4.30+4.46 / 72.5$ & $3.95+4.21 / 72.6$ & $4.34 / 72.3$ & $3.73+3.96 / 71.6$ \\
\hline $\mathrm{H}-7 " \mathrm{a}+\mathrm{H}-7$ "b/C-7" & $4.27+4.76 / 73.0$ & $4.58+4.66 / 74.4$ & $4.50+5.07 / 73.9$ & $4.59+4.67 / 74.2$ \\
\hline C-8 & 135.9 & 135.3 & 135.4 & 135.4 \\
\hline C-8' & 136.9 & 136.1 & 136.3 & 136.5 \\
\hline C-8" & 135.8 & 136.5 & 135.9 & 136.6 \\
\hline $\mathrm{H}-9 / \mathrm{C}-9$ & $7.47 / 126.1$ & $7.62 / 127.0$ & $7.36 / 126.7$ & $7.78 / 128.5$ \\
\hline H-9'/C-9' & $7.51 / 124.5$ & $7.47 / 126.0$ & $7.40 / 124.4$ & $7.16 / 123.1$ \\
\hline $\mathrm{H}-9 " / \mathrm{C}-9 "$ & $7.64 / 126.2$ & $7.49 / 125.5$ & $7.83 / 127.4$ & $7.62 / 125.1$ \\
\hline C-10 & $132.78 / 132.0$ & 132.6 & nd & 132.8 \\
\hline C-10' & 132.6 & 132.4 & nd & 132.2 \\
\hline C-10" & 132.6 & $132.82 / 132.3$ & nd & 132.8 \\
\hline $\mathrm{H}-11 / \mathrm{C}-11$ & $7.51 / 127.8$ & $7.65 / 127.8$ & $7.30 / 127.8$ & $7.67 / 128.0$ \\
\hline $\mathrm{H}-11^{\prime} / \mathrm{C}-11^{\prime}$ & $7.46 / 126.1$ & $7.55 / 127.6$ & 7.44/127.2 & $7.18 / 126.4$ \\
\hline $\mathrm{H}-11 " / \mathrm{C}-11 "$ & $7.65 / 128.1$ & $7.23 / 125.3$ & $7.76 / 128.4$ & $7.49 / 128.8$ \\
\hline $\mathrm{H}-12 / \mathrm{C}-12$ & $7.16 / 125.4$ & $7.27 / 125.3$ & $7.21 / 125.9$ & $7.14 / 127.2$ \\
\hline $\mathrm{H}-12^{\prime} / \mathrm{C}-12$ & $7.11 / 125.6$ & $7.32 / 125.8$ & $7.17 / 126.6$ & $7.12 / 128.0$ \\
\hline $\mathrm{H}-12 " / \mathrm{C}-12 "$ & $7.35 / 124.8$ & $6.87 / 125.4$ & $7.38 / 125.5$ & 7.32/123.7 \\
\hline C-13 & 134.2 & 135.1 & 134.3 & 134.2 \\
\hline C-13' & 133.3 & 133.5 & 133.6 & 131.1 \\
\hline C-13" & 135.3 & 134.7 & 134.9 & 135.2 \\
\hline $\mathrm{H}-14 / \mathrm{C}-14$ & $7.46 / 126.3$ & $7.57 / 125.9$ & $7.45 / 126.6$ & $7.58 / 128.1$ \\
\hline H-14'/C-14' & $6.94 / 126.8$ & $7.06 / 126.3$ & $6.84 / 128.2$ & $6.55 / 129.9$ \\
\hline H-14"/C-14" & $7.77 / 125.4$ & $7.56 / 126.3$ & $7.73 / 126.3$ & 7.70/124.1 \\
\hline C-15 & $132.78 / 132.0$ & 132.8 & nd & 132.8 \\
\hline C-15' & 132.5 & 132.2 & nd & 131.1 \\
\hline C-15" & 132.6 & $132.82 / 132.3$ & nd & 132.5 \\
\hline $\mathrm{H}-16 / \mathrm{C}-16$ & 7.39/127.7 & $7.55 / 128.2$ & $7.23 / 128.0$ & 7.69/128.2 \\
\hline $\mathrm{H}-16^{\prime} / \mathrm{C}-16^{\prime}$ & $6.68 / 127.7$ & $6.79 / 127.6$ & $6.98 / 127.9$ & $6.14 / 126.9$ \\
\hline H-16"/C-16" & $7.58 / 127.9$ & 7.52/127.7 & 7.79/128.0 & 7.49/127.6 \\
\hline $\mathrm{H}-17 / \mathrm{C}-17$ & $7.02 / 126.2$ & $7.38 / 126.5$ & $7.02 / 126.3$ & $7.52 / 128.2$ \\
\hline $\mathrm{H}-17^{\prime} / \mathrm{C}-17^{\prime}$ & $6.88 / 125.6$ & $6.91 / 126.3$ & $7.01 / 125.0$ & $6.49 / 124.9$ \\
\hline H-17"/C-17" & $7.36 / 126.3$ & $7.04 / 125.4$ & 7.48/127.2 & $7.21 / 125.3$ \\
\hline $\mathrm{H}-18 \mathrm{a}+\mathrm{H}-18 \mathrm{~b} / \mathrm{C}-18$ & $4.81+5.24 / 71.4$ & $4.87+5.09 / 71.3$ & $5.31+5.40 / 70.2$ & $3.94+4.87 / 70.6$ \\
\hline $\mathrm{H}-18^{\prime} \mathrm{a}+\mathrm{H}-18^{\prime} \mathrm{b} / \mathrm{C}-18^{\prime}$ & $5.15+5.26 / 72.6$ & $5.35+5.46 / 72.3$ & $4.40+5.20 / 76.2$ & $5.00+5.35 / 77.0$ \\
\hline $\mathrm{H}-18$ "a+H-18"b/C-18" & $5.15+5.36 / 72.7$ & $4.94+5.27 / 73.9$ & $4.97+5.21 / 71.3$ & $5.45+5.64 / 69.4$ \\
\hline CTV-ring A & & & & \\
\hline C-19 & & 147.3 & 145.8 & 147.1 \\
\hline C-20 & 148.5 & 148.4 & 148.6 & 147.6 \\
\hline H-21/C-21 & $6.64 / 113.0$ & $6.70 / 113.5$ & $6.99 / 113.8$ & $6.54 / 111.8$ \\
\hline C-22 & 132.7 & 132.8 & 133.2 & 130.9 \\
\hline
\end{tabular}




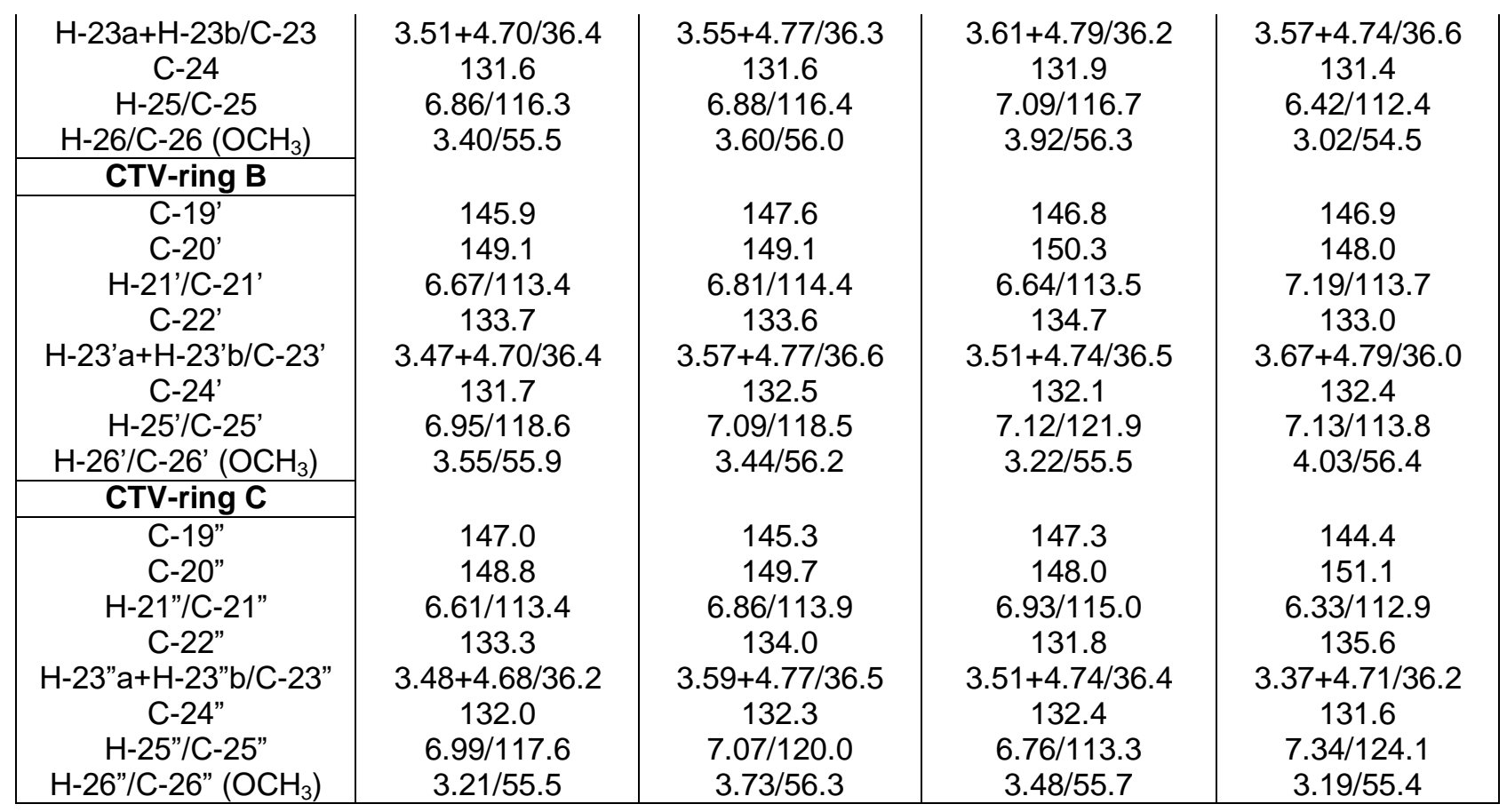




\section{Fluorescence titration experiments}

Fluorescence titration curves for acetylcholine iodide
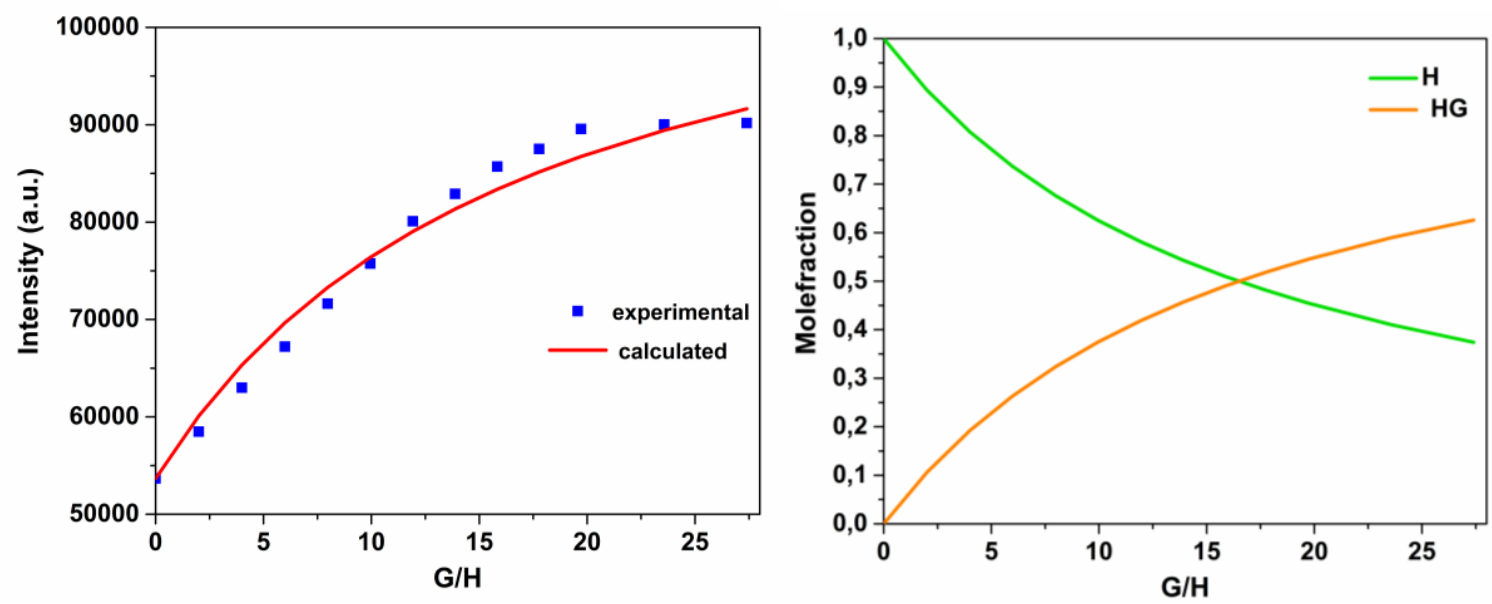

FigureS1. Fluorescence intensity and molefraction as a functions of guest/host ratio for cage P-5a.
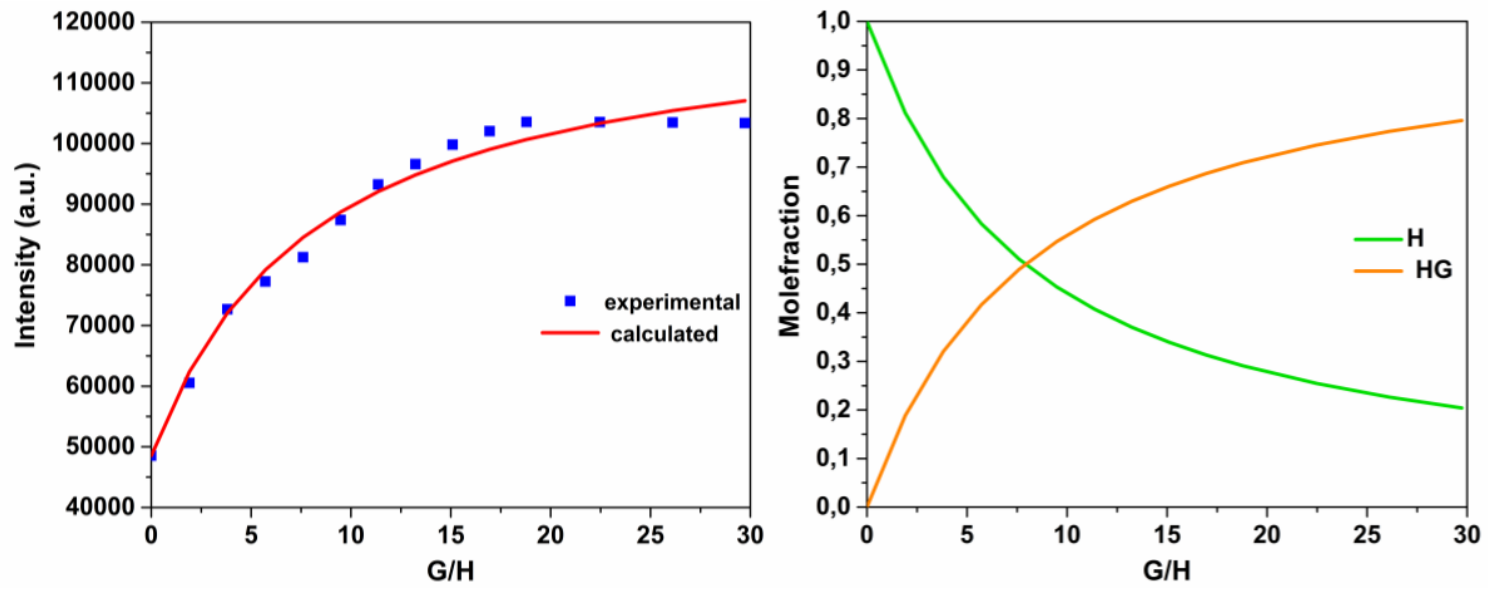

FigureS2. Fluorescence intensity and molefraction as a functions of guest/host ratio for cage M-5a.

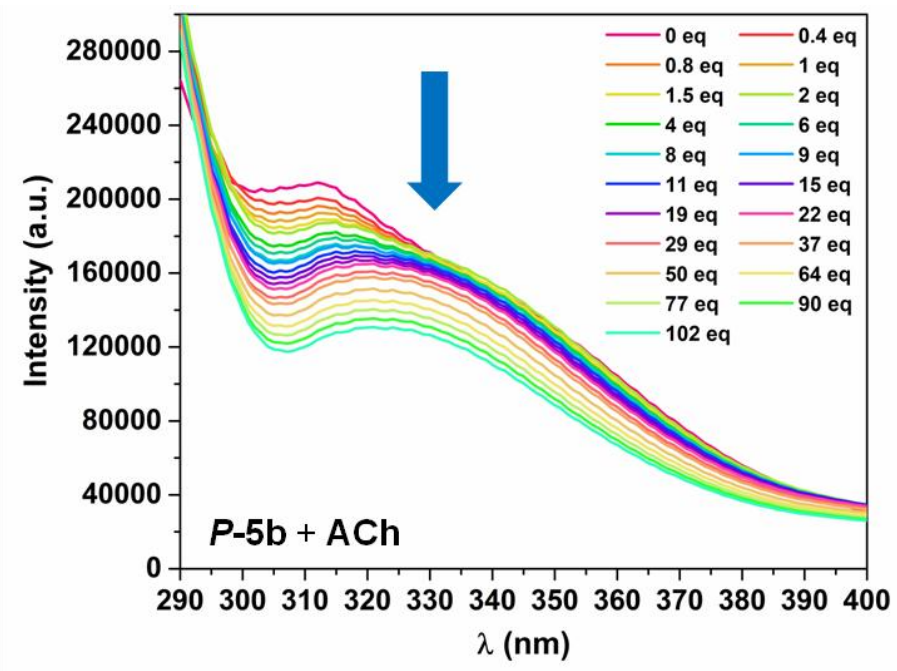

FigureS3. Fluorescence titration of host $\boldsymbol{P}$-5b with $\mathrm{ACh}$ in $\mathrm{CH}_{3} \mathrm{CN}$ excited at $280 \mathrm{~nm}$. 

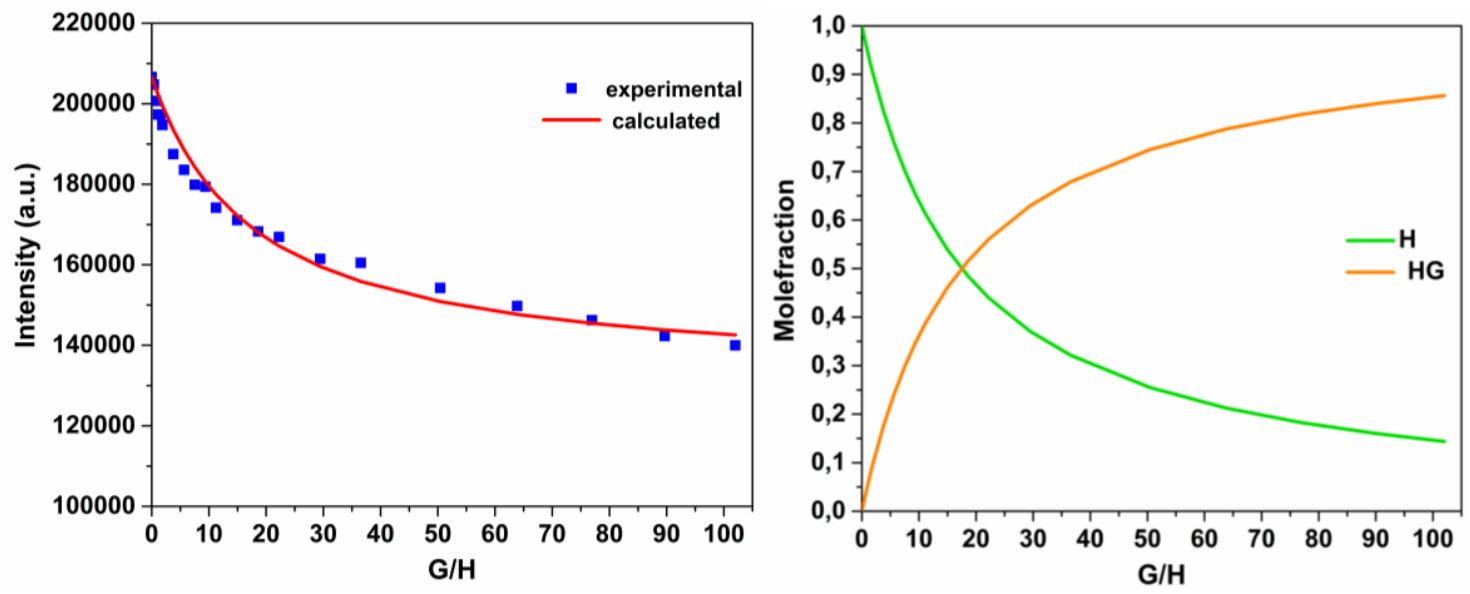

FigureS4. Fluorescence intensity and molefraction as a functions of guest/host ratio for cage P-5b.

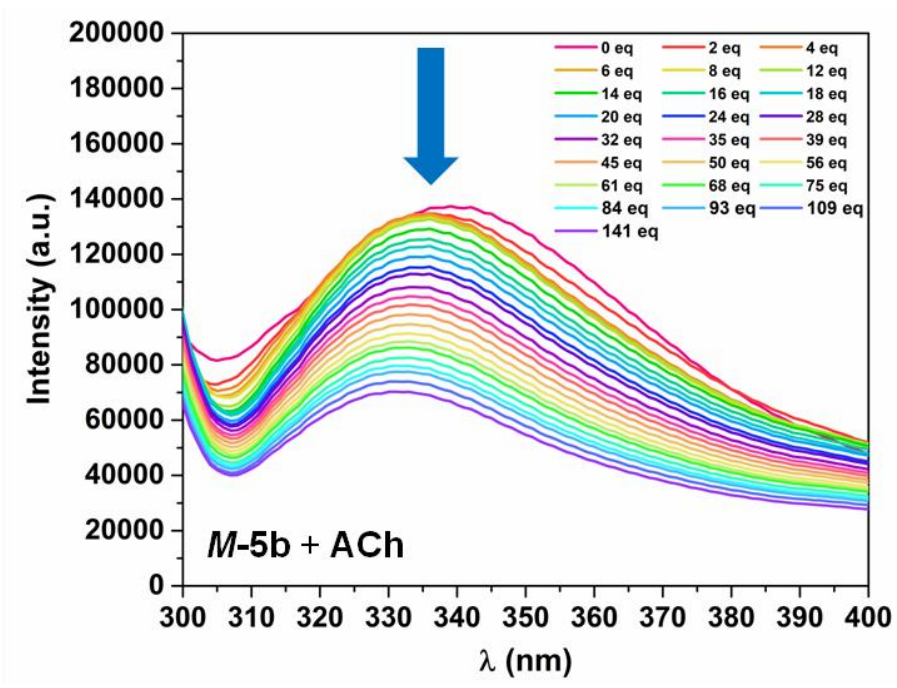

Figure S5. Fluorescence titration of host $\boldsymbol{M}-\mathbf{5 b}$ with $\mathrm{ACh}$ in $\mathrm{CH}_{3} \mathrm{CN}$ excited at $280 \mathrm{~nm}$.
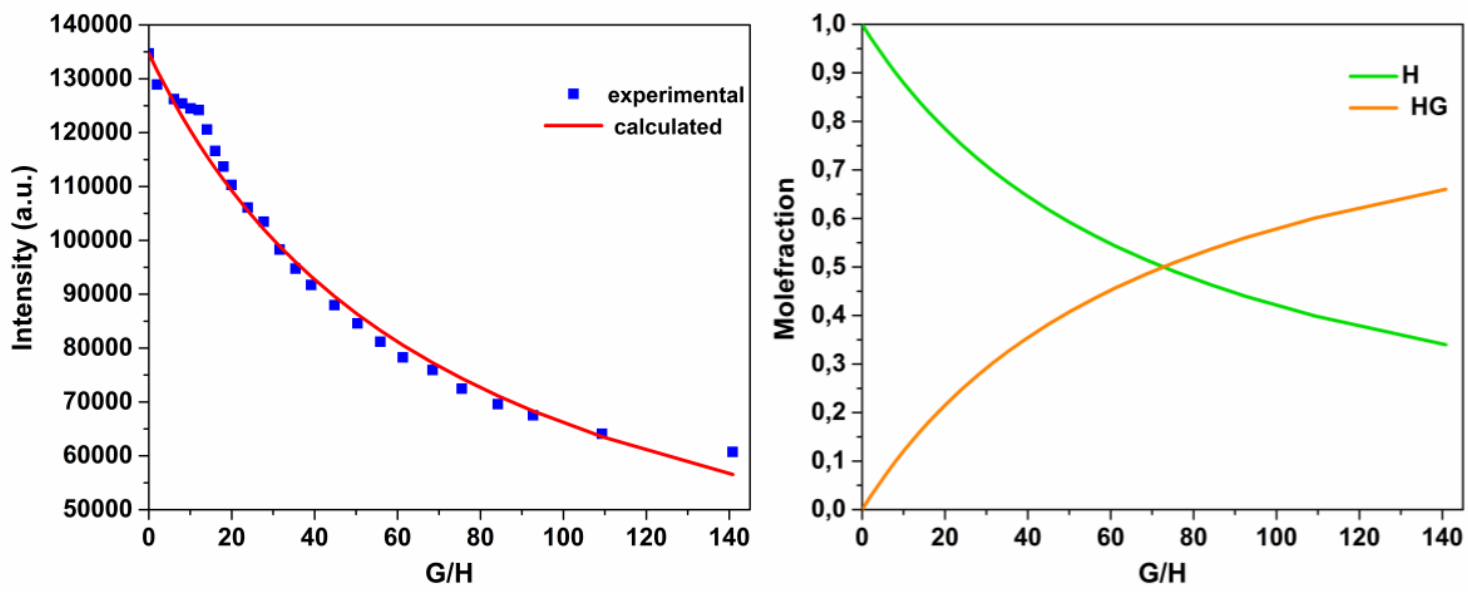

Figure S6. Fluorescence intensity and molefraction as a functions of guest/host ratio for cage M-5b. 


\section{Fluorescence titration curves for choline iodide}
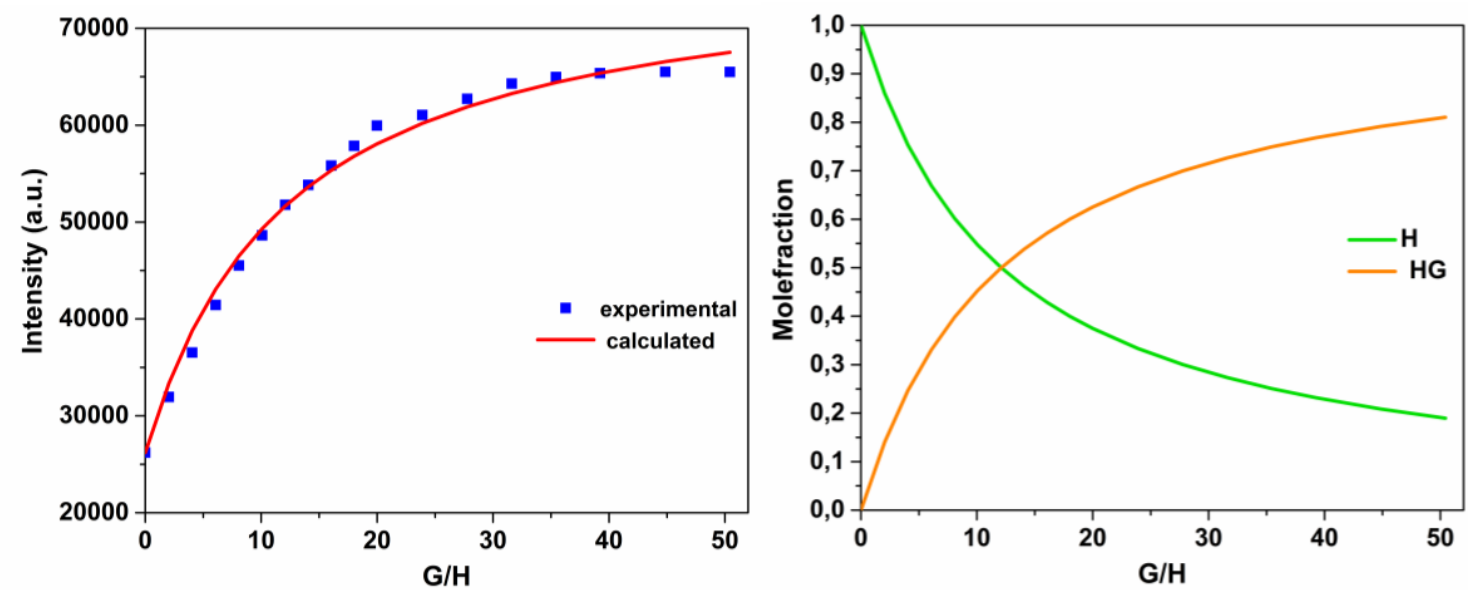

Figure S7. Fluorescence intensity and molefraction as a functions of guest/host ratio for cage

$P-5$.
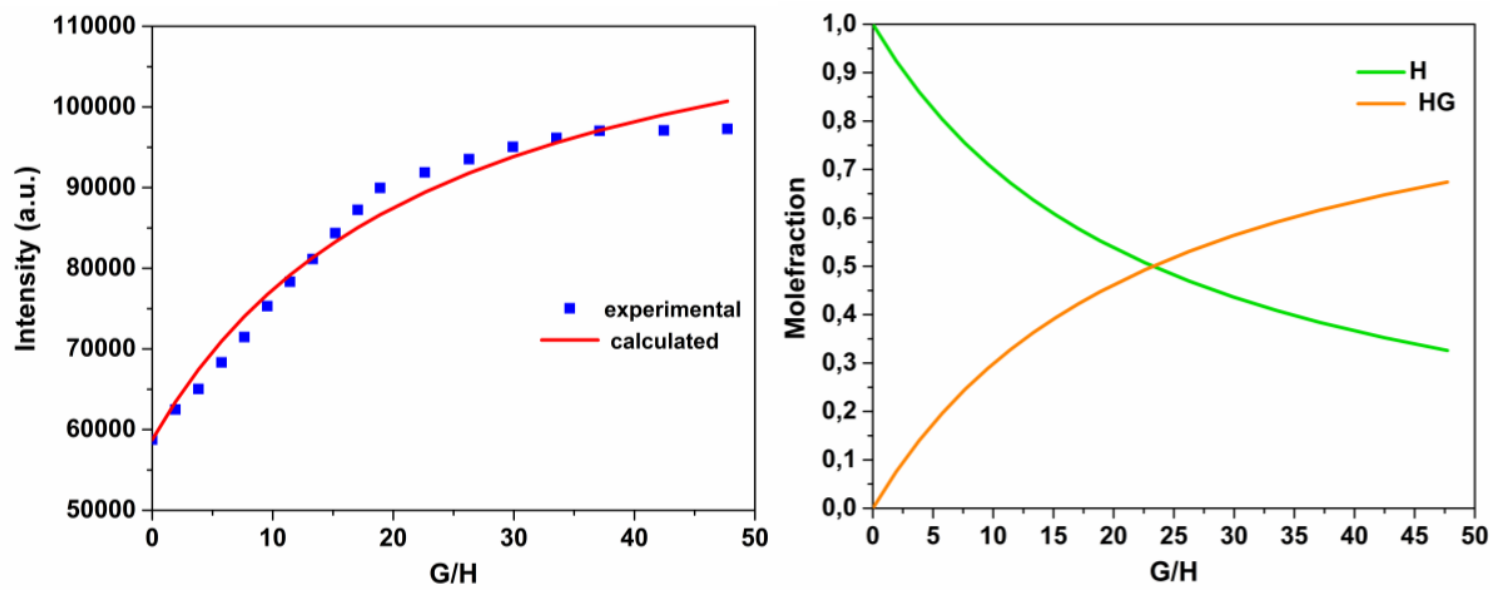

Figure S8. Fluorescence intensity and molefraction as a functions of guest/host ratio for cage M-5a.

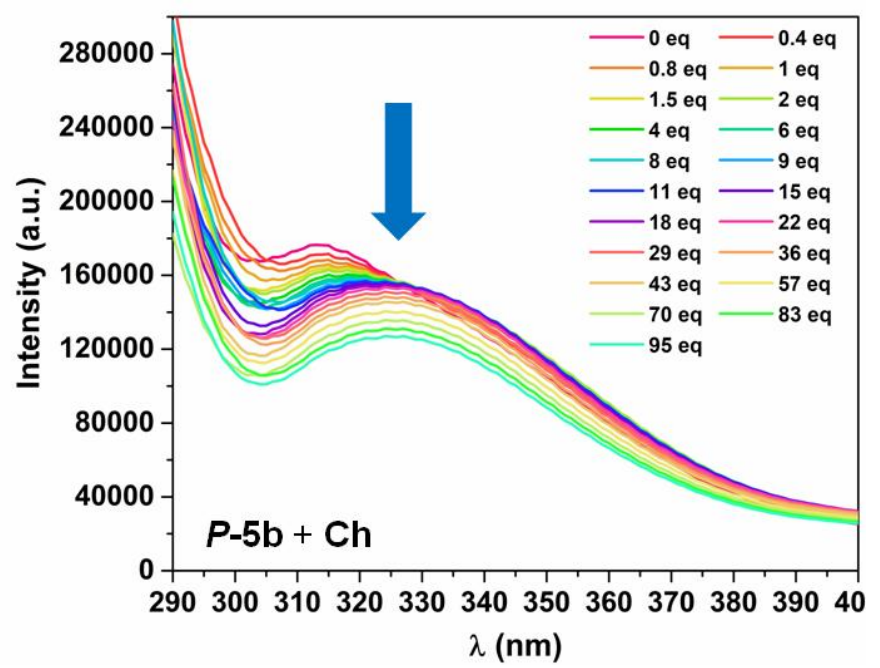

Figure S9. Fluorescence titration of host $\boldsymbol{P}-5 \mathbf{b}$ with $\mathrm{Ch}$ in $\mathrm{CH}_{3} \mathrm{CN}$ excited at $280 \mathrm{~nm}$. 

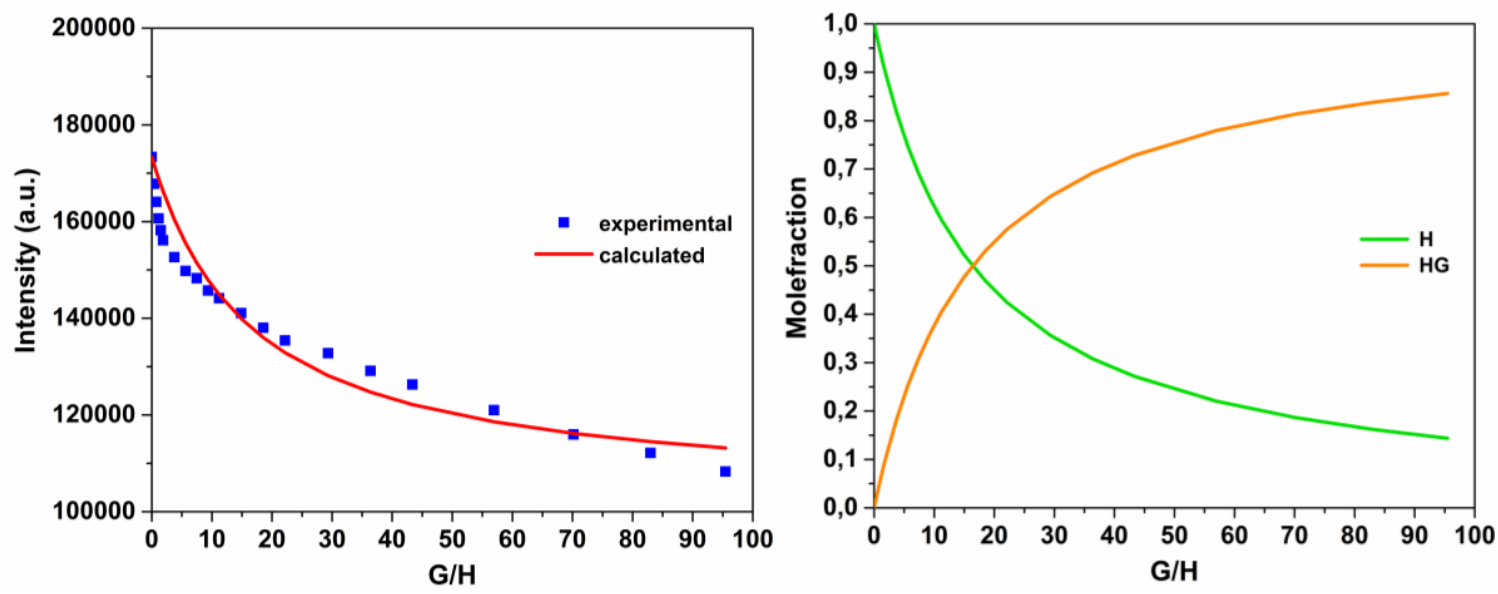

Figure S10. Fluorescence intensity and molefraction as a functions of guest/host ratio for cage P-5b.

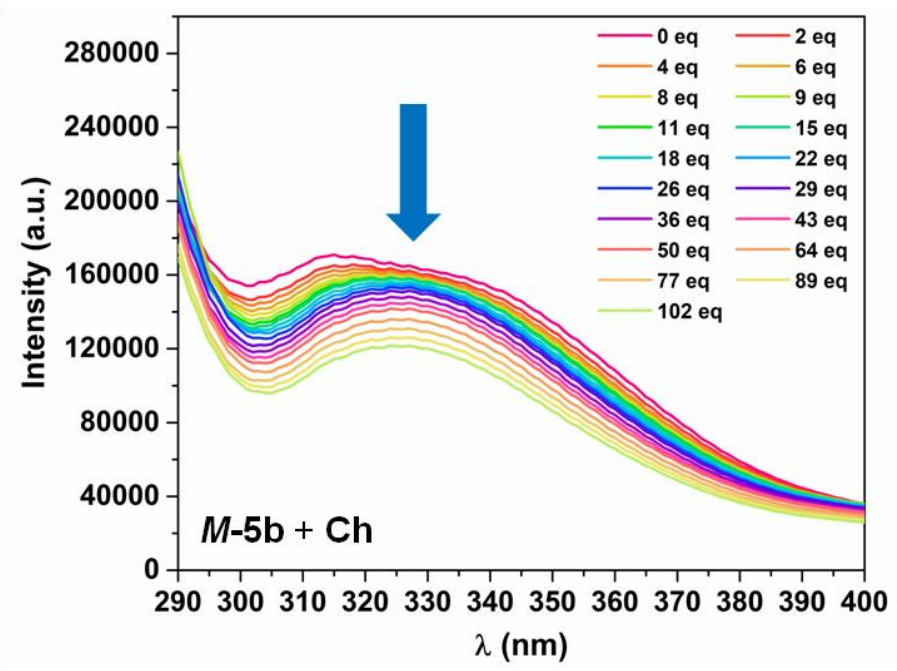

Figure S11. Fluorescence titration of host $\boldsymbol{M}-5 \mathbf{b}$ with $\mathrm{Ch}$ in $\mathrm{CH}_{3} \mathrm{CN}$ excited at $280 \mathrm{~nm}$.
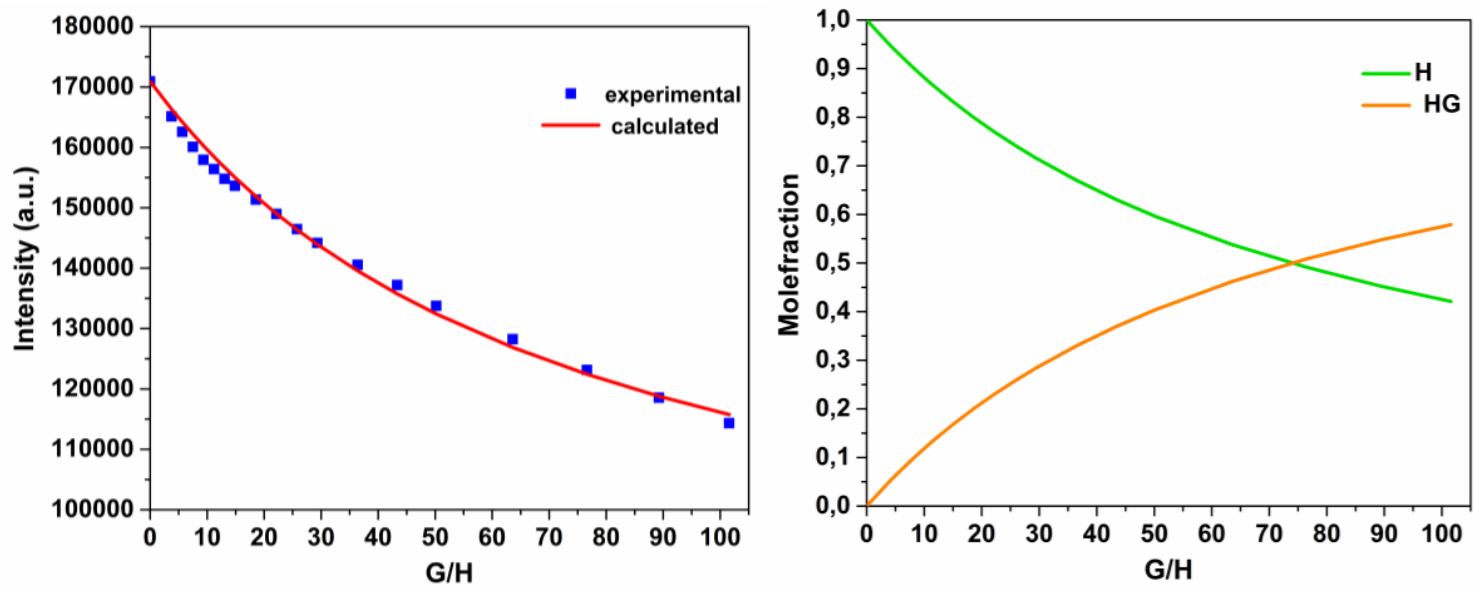

Figure S12. Fluorescence intensity and molefraction as a functions of guest/host ratio for cage M-5b. 


\section{Fit parameters}

Table S2. Fit parameters obtained from the fluorescence titrations.

\begin{tabular}{|c|c|c|c|c|}
\hline Host & Guest & $\boldsymbol{K}_{\boldsymbol{a}}\left(\mathrm{M}^{-1}\right)$ & RMS (Intensity a.u.) & Covariance \\
\hline \multirow{2}{*}{$\boldsymbol{P}-5 \mathrm{a}$} & ACh & $2.2 \times 10^{3} \pm 1.6 \%$ & $1.0 \times 10^{3}$ & $2.1 \times 10^{-2}$ \\
\cline { 2 - 5 } & Ch & $3.8 \times 10^{3} \pm 0.9 \%$ & $1.6 \times 10^{3}$ & $8.5 \times 10^{-3}$ \\
\hline \multirow{2}{*}{ M-5a } & ACh & $5.6 \times 10^{3} \pm 1.7 \%$ & $2.2 \times 10^{3}$ & $1.8 \times 10^{-2}$ \\
\cline { 2 - 5 } & Ch & $1.8 \times 10^{3} \pm 1.4 \%$ & $1.8 \times 10^{3}$ & $2.2 \times 10^{-2}$ \\
\hline \multirow{2}{*}{$\boldsymbol{P}$-5b } & ACh & $2.4 \times 10^{3} \pm 3.5 \%$ & $6.1 \times 10^{3}$ & $7.2 \times 10^{-2}$ \\
\cline { 2 - 5 } & Ch & $2.6 \times 10^{3} \pm 3.8 \%$ & $2.6 \times 10^{3}$ & $2.8 \times 10^{-2}$ \\
\hline \multirow{2}{*}{ M-5b } & ACh & $0.6 \times 10^{3} \pm 0.9 \%$ & $2.8 \times 10^{3}$ & $1.7 \times 10^{-2}$ \\
\cline { 2 - 5 } & Ch & $0.5 \times 10^{3} \pm 1.1 \%$ & $2.6 \times 10^{3}$ & $2.8 \times 10^{-2}$ \\
\hline
\end{tabular}

\section{${ }^{1} \mathrm{H}$ NMR titration experiments}

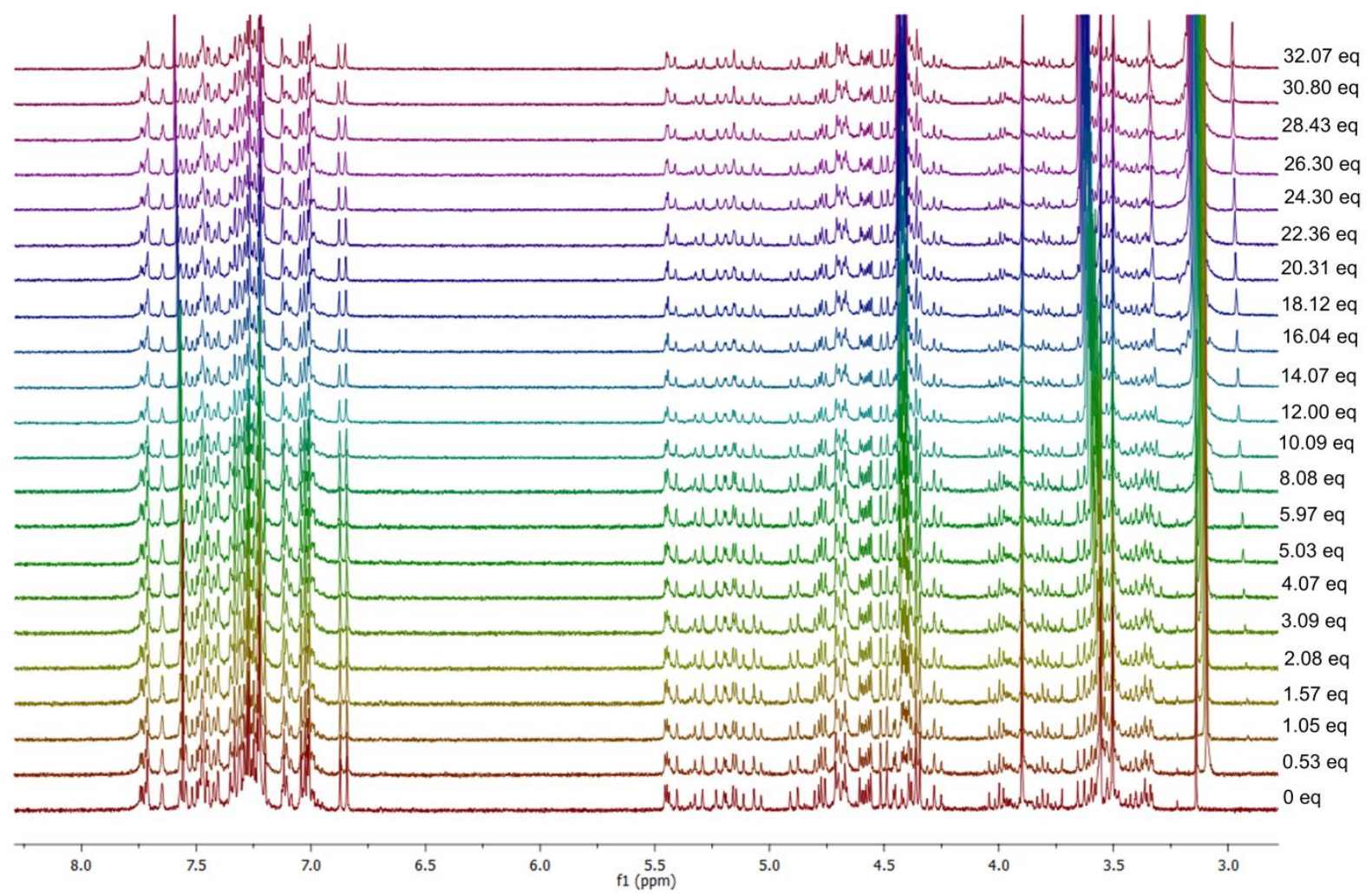

Figure S13. ${ }^{~} \mathrm{H}$ NMR $\left(400 \mathrm{MHz}, \mathrm{CD}_{3} \mathrm{CN} / \mathrm{CDCl}_{3}=80: 20\right)$ spectra of $\boldsymbol{M}$-5a after gradual addition of acetylcholine iodide in the same solvent. 


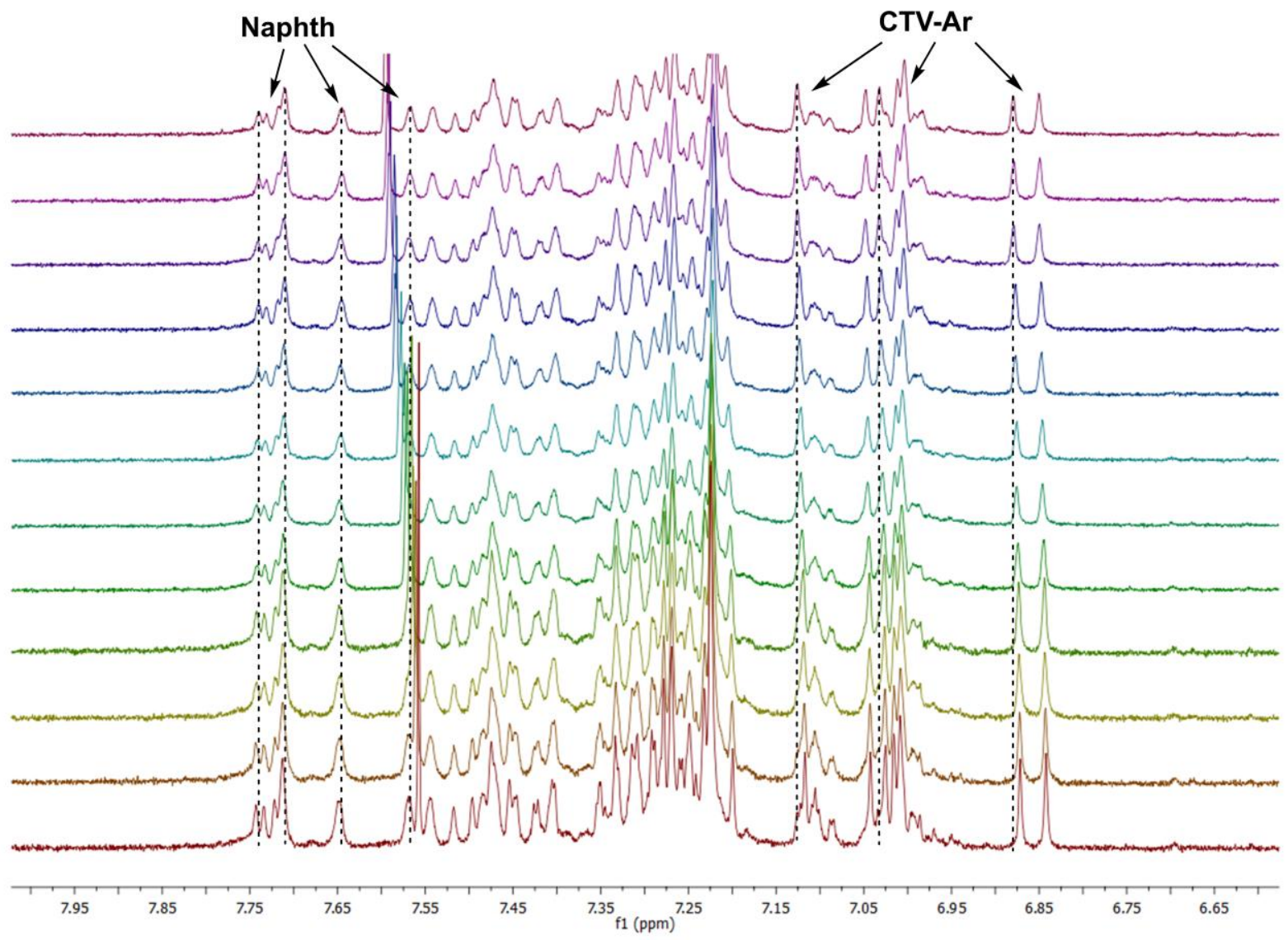

Figure S14. ${ }^{1} \mathrm{H}$ NMR $\left(400 \mathrm{MHz}, \mathrm{CD}_{3} \mathrm{CN} / \mathrm{CDCl}_{3}=80: 20\right)$ spectra of aromatic part of $\boldsymbol{M}$-5a after gradual addition of acetylcholine iodide in the same solvent.

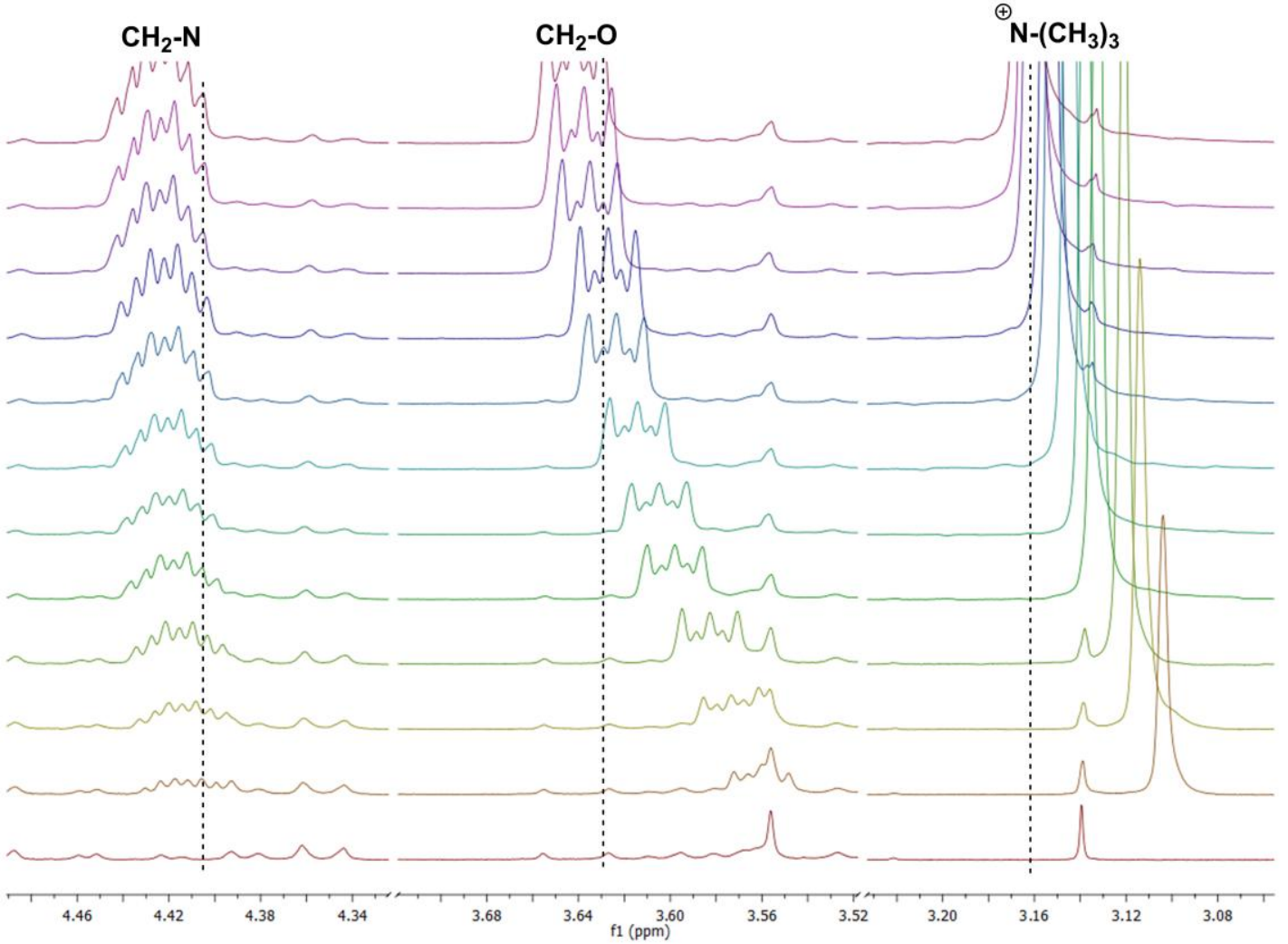

Figure S15. ${ }^{1} \mathrm{H}$ NMR (400 MHz, $\left.\mathrm{CD}_{3} \mathrm{CN} / \mathrm{CDCl}_{3}=80: 20\right)$ spectra of $\mathrm{ACh}$ chemical shifts during titration studies of $\boldsymbol{M}-5 \mathrm{a}$ with acetylcholine iodide. 


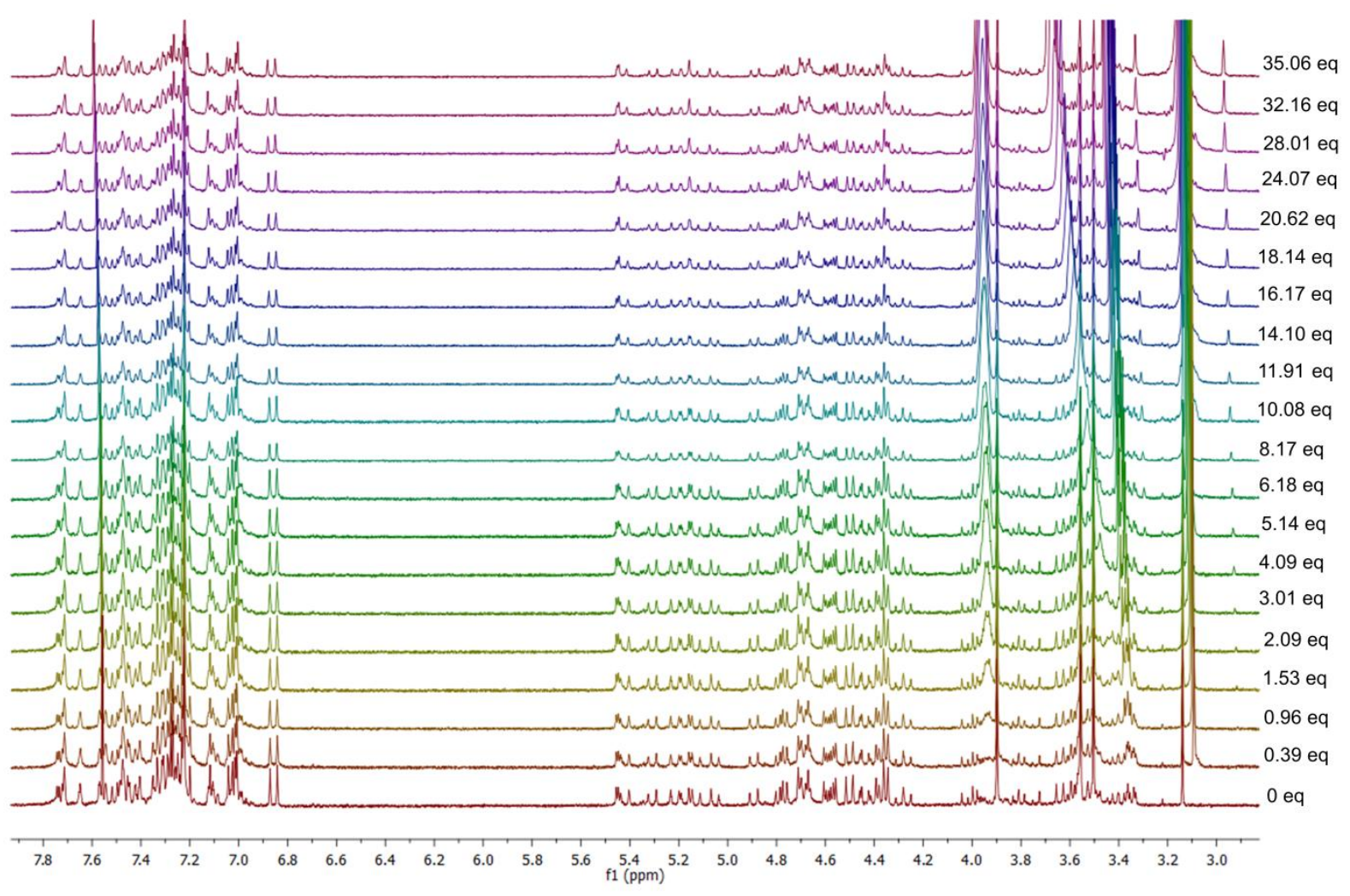

Figure S16. ${ }^{1} \mathrm{H}$ NMR $\left(400 \mathrm{MHz}, \mathrm{CD}_{3} \mathrm{CN} / \mathrm{CDCl}_{3}=80: 20\right)$ spectra of $\boldsymbol{M}$-5a after gradual addition of choline iodide in the same solvent.

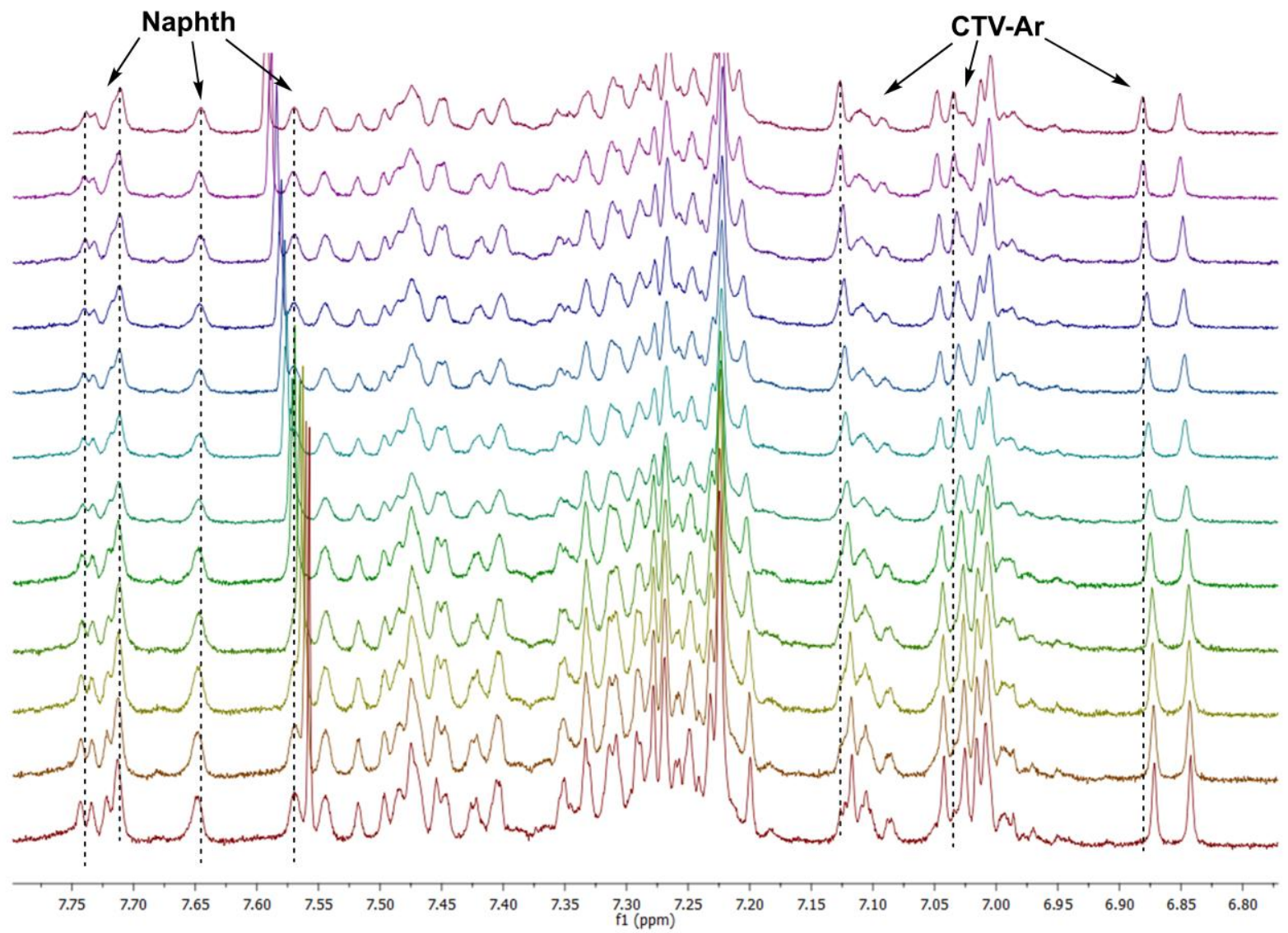

Figure S17. ${ }^{1} \mathrm{H}$ NMR $\left(400 \mathrm{MHz}, \mathrm{CD}_{3} \mathrm{CN} / \mathrm{CDCl}_{3}=80: 20\right)$ spectra of aromatic part of $\boldsymbol{M}-\mathbf{5 a}$ after gradual addition of choline iodide in the same solvent. 


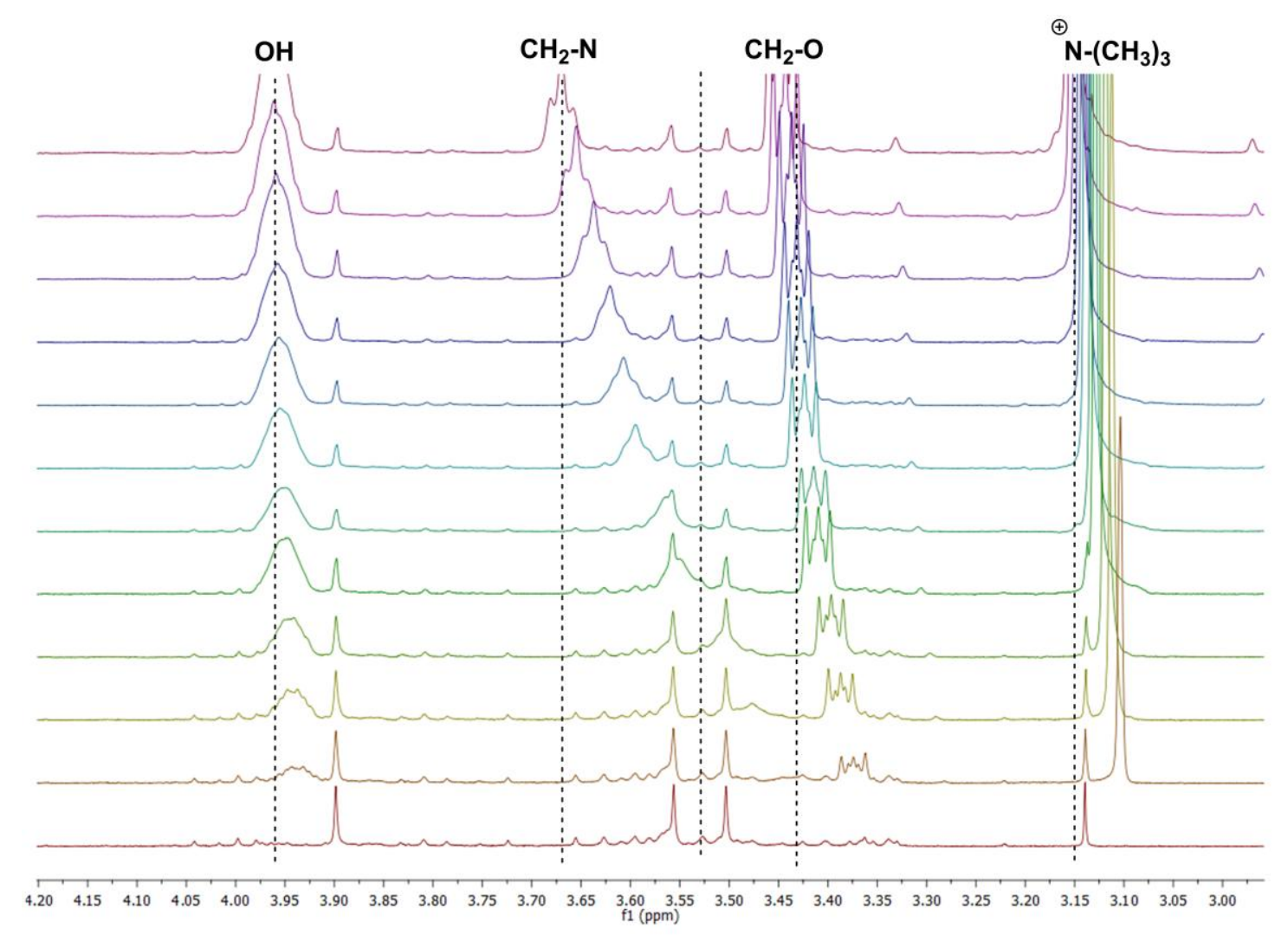

Figure S18. ${ }^{1} \mathrm{H} \mathrm{NMR}\left(400 \mathrm{MHz}, \mathrm{CD}_{3} \mathrm{CN} / \mathrm{CDCl}_{3}=80: 20\right)$ spectra of $\mathrm{Ch}$ chemical shifts during titration studies of $\boldsymbol{M}-\mathbf{5 a}$ with choline iodide.

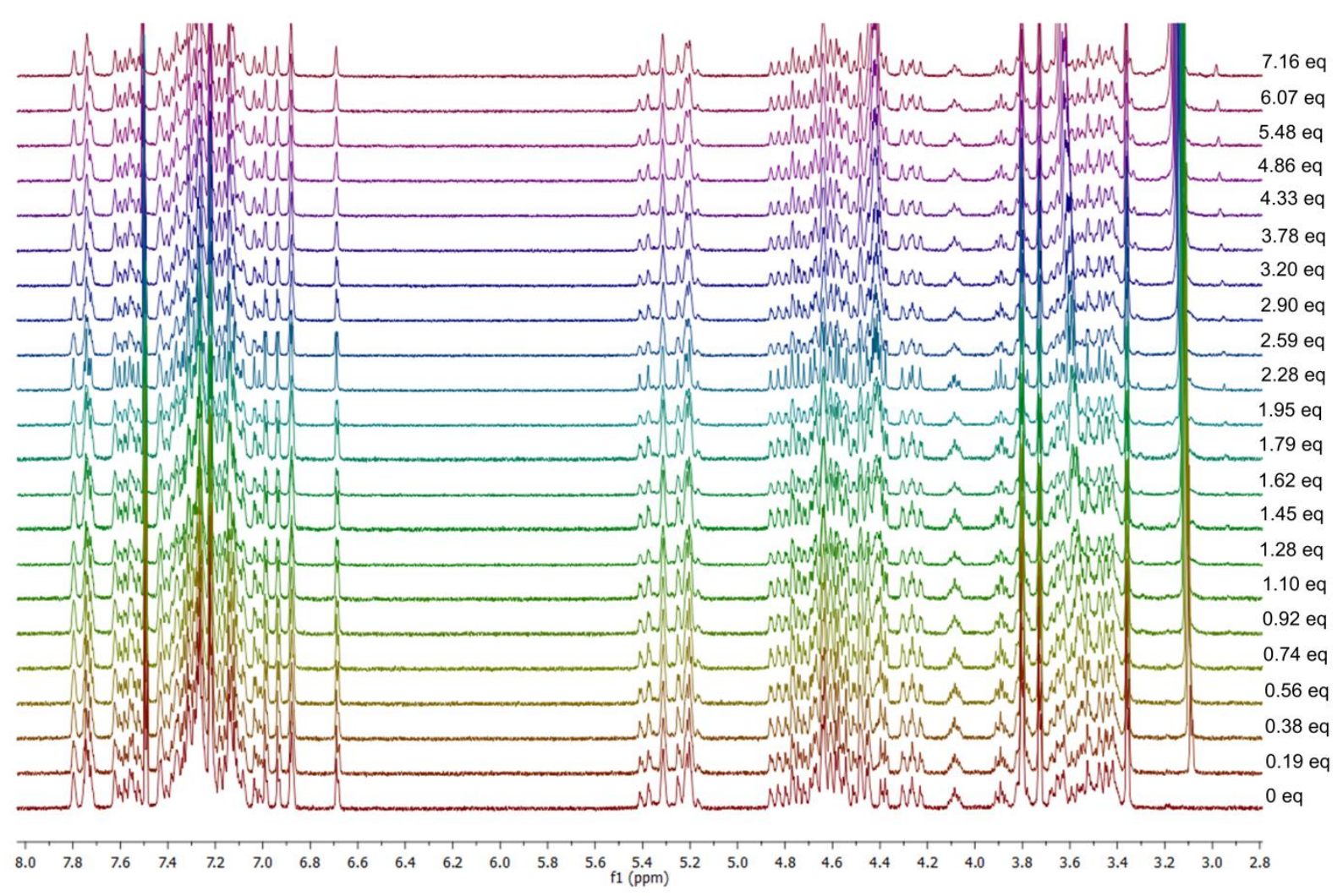

Figure S19. ${ }^{1} \mathrm{H}$ NMR (400 MHz, $\mathrm{CD}_{3} \mathrm{CN} / \mathrm{CDCl}_{3}=80: 20$ ) spectra of $\boldsymbol{P}$-5a after gradual addition of acetylcholine iodide in the same solvent. 


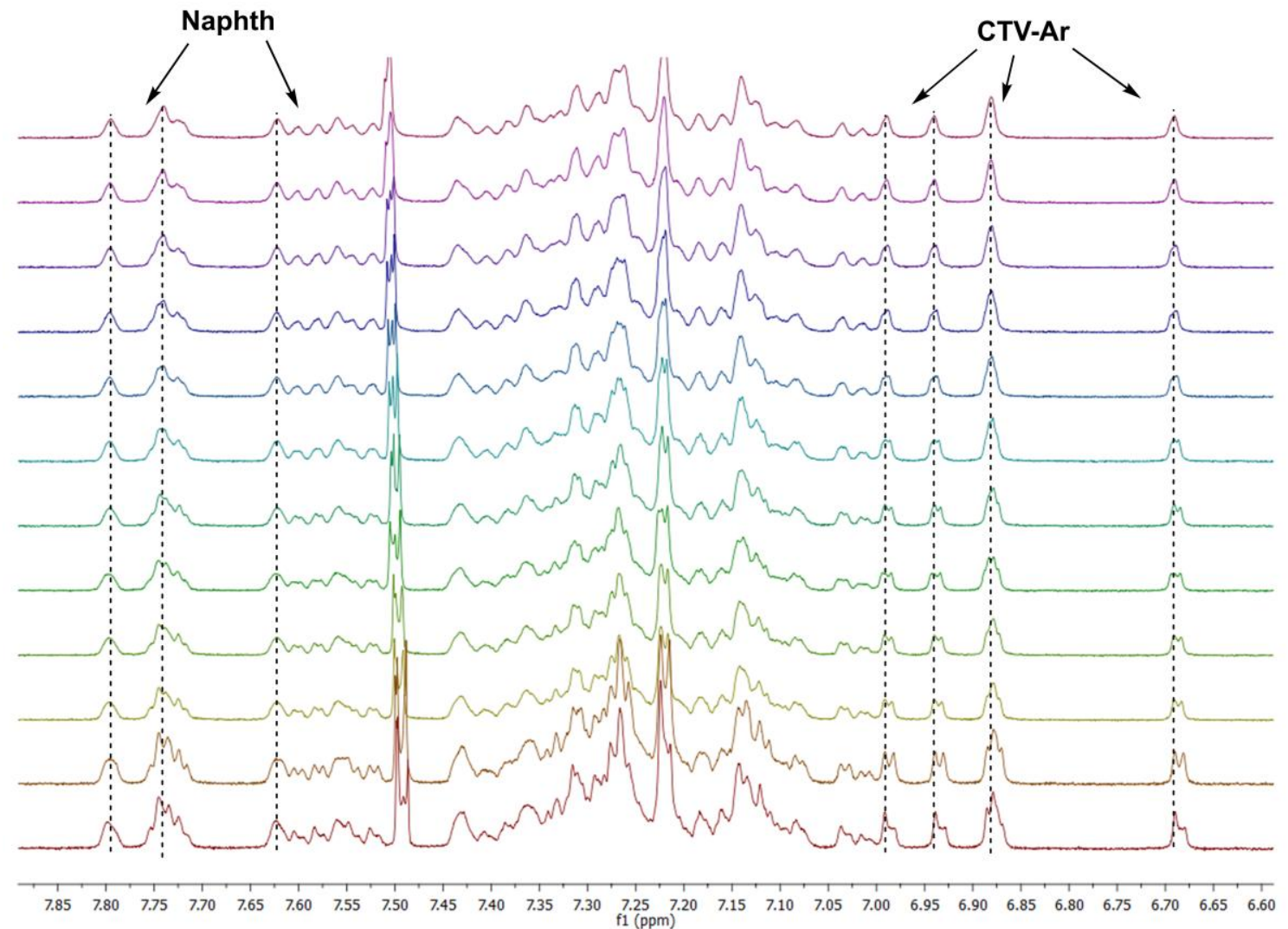

Figure S20. ${ }^{1} \mathrm{H}$ NMR (400 MHz, $\left.\mathrm{CD}_{3} \mathrm{CN} / \mathrm{CDCl}_{3}=80: 20\right)$ spectra of aromatic part of $\boldsymbol{P}$-5a after gradual addition of acetylcholine iodide in the same solvent.

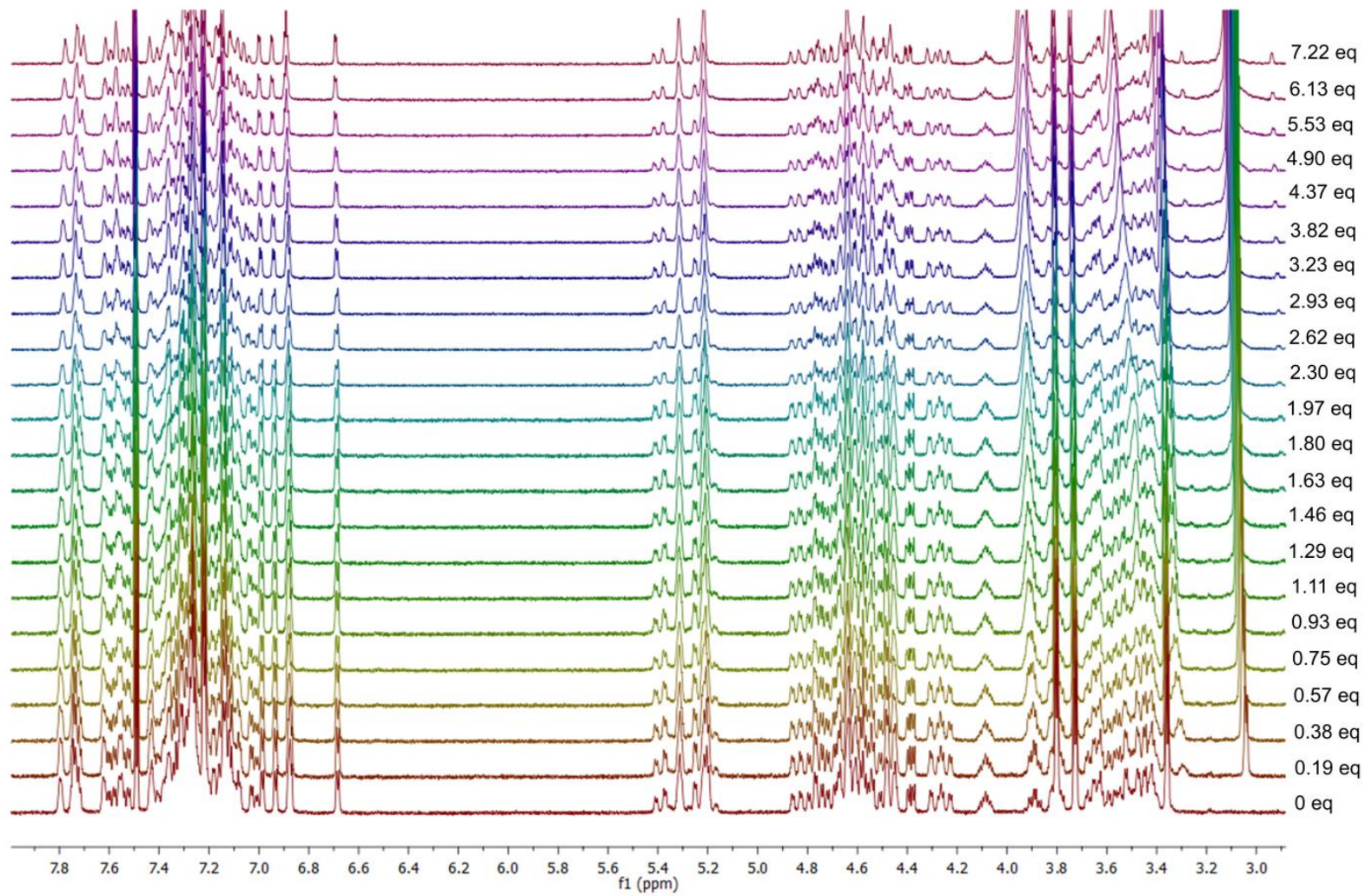

Figure S21. ${ }^{1} \mathrm{H}$ NMR $\left(400 \mathrm{MHz}, \mathrm{CD}_{3} \mathrm{CN} / \mathrm{CDCl}_{3}=80: 20\right)$ spectra of $\boldsymbol{P}-5$ a after gradual addition of choline iodide in the same solvent. 


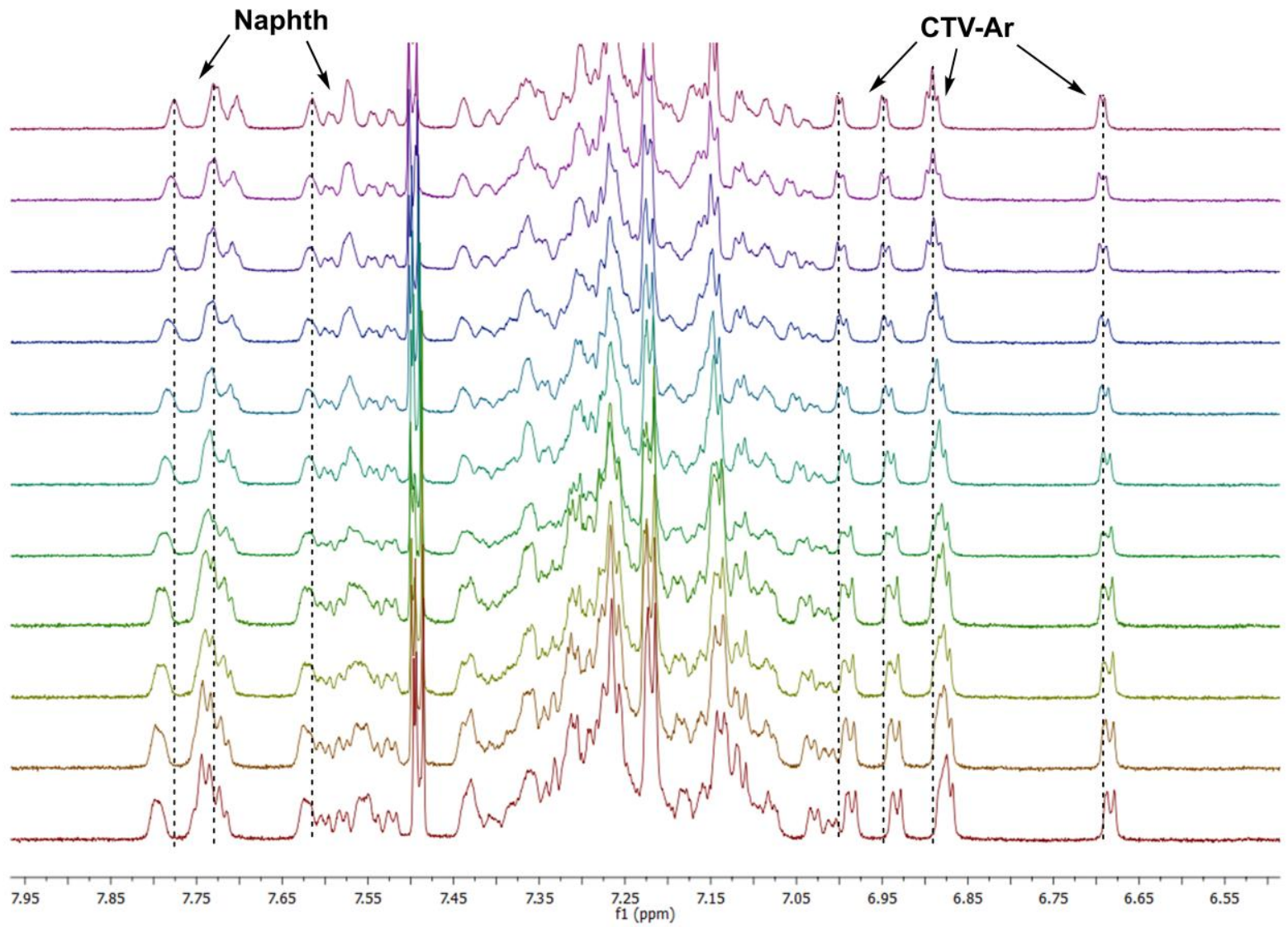

Figure S22. ${ }^{1} \mathrm{H}$ NMR $\left(400 \mathrm{MHz}, \mathrm{CD}_{3} \mathrm{CN} / \mathrm{CDCl}_{3}=80: 20\right)$ spectra of aromatic part of $\boldsymbol{P}$-5a after gradual addition of choline iodide in the same solvent. 


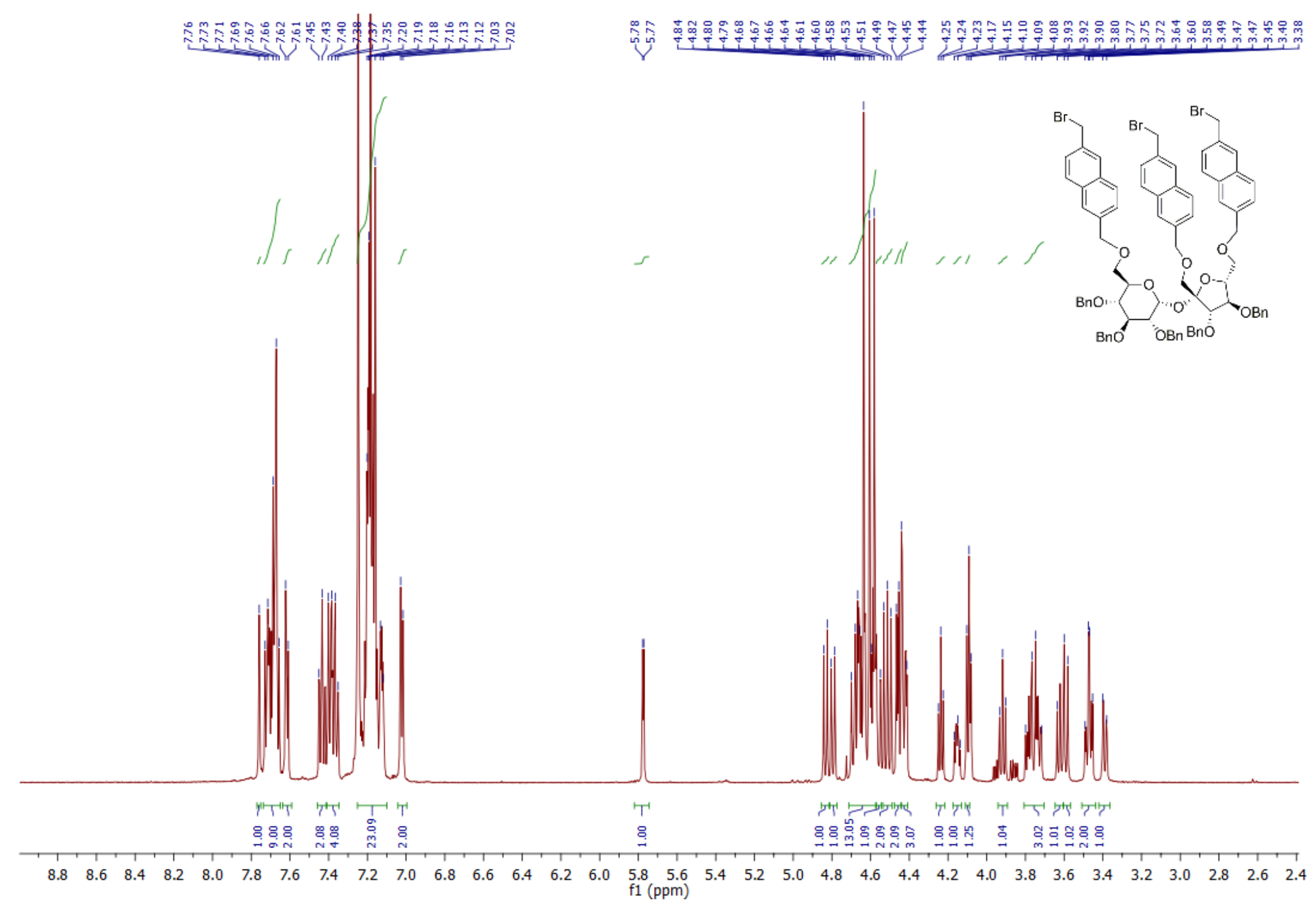

Figure S23. ${ }^{1} \mathrm{H}$ NMR $\left(600 \mathrm{MHz}, \mathrm{CDCl}_{3}\right)$ spectrum of compound 3. 


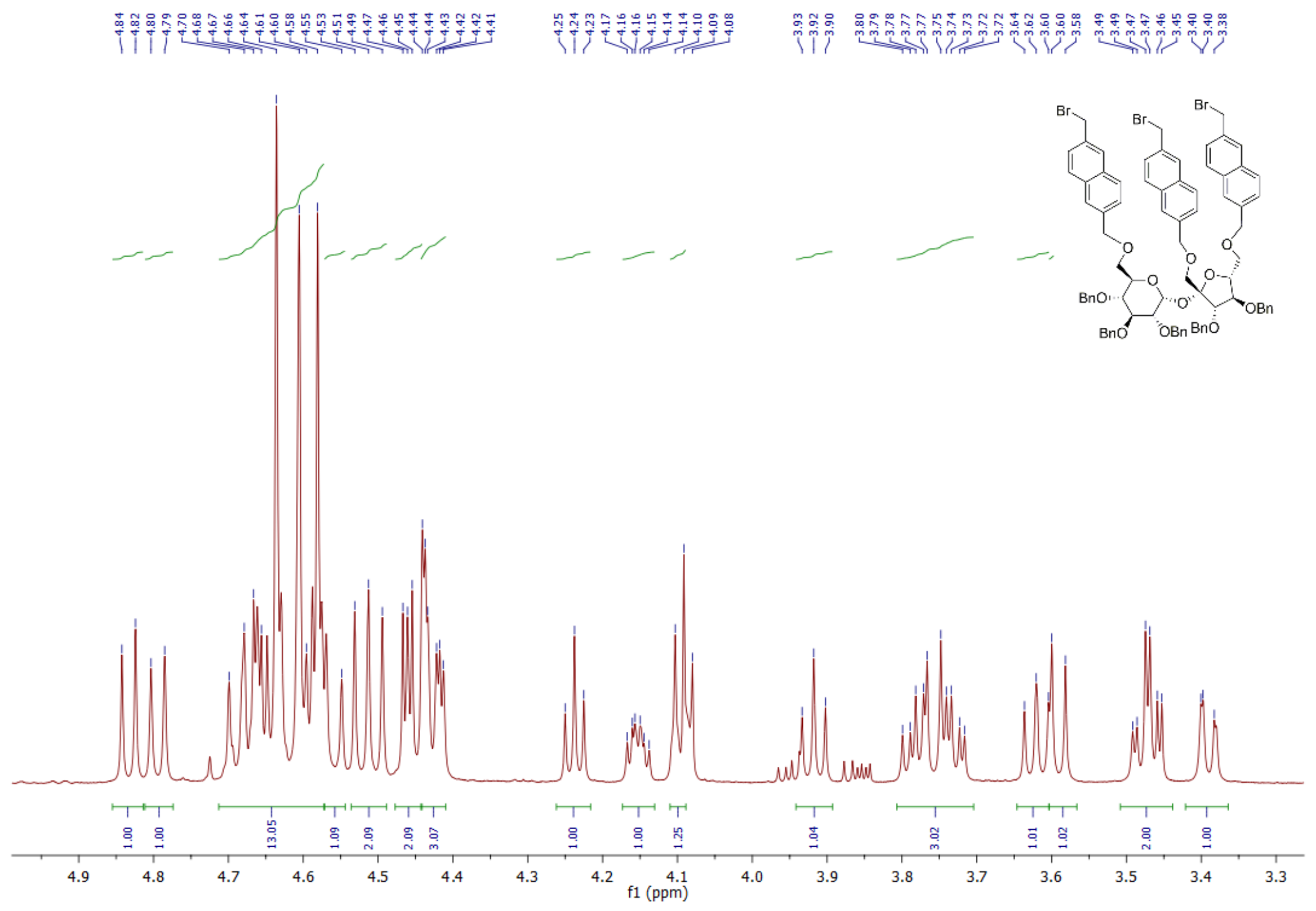

Figure S24. ${ }^{1} \mathrm{H} \mathrm{NMR}\left(600 \mathrm{MHz}, \mathrm{CDCl}_{3}\right)$ spectrum of compound 3 (aliphatic part). 


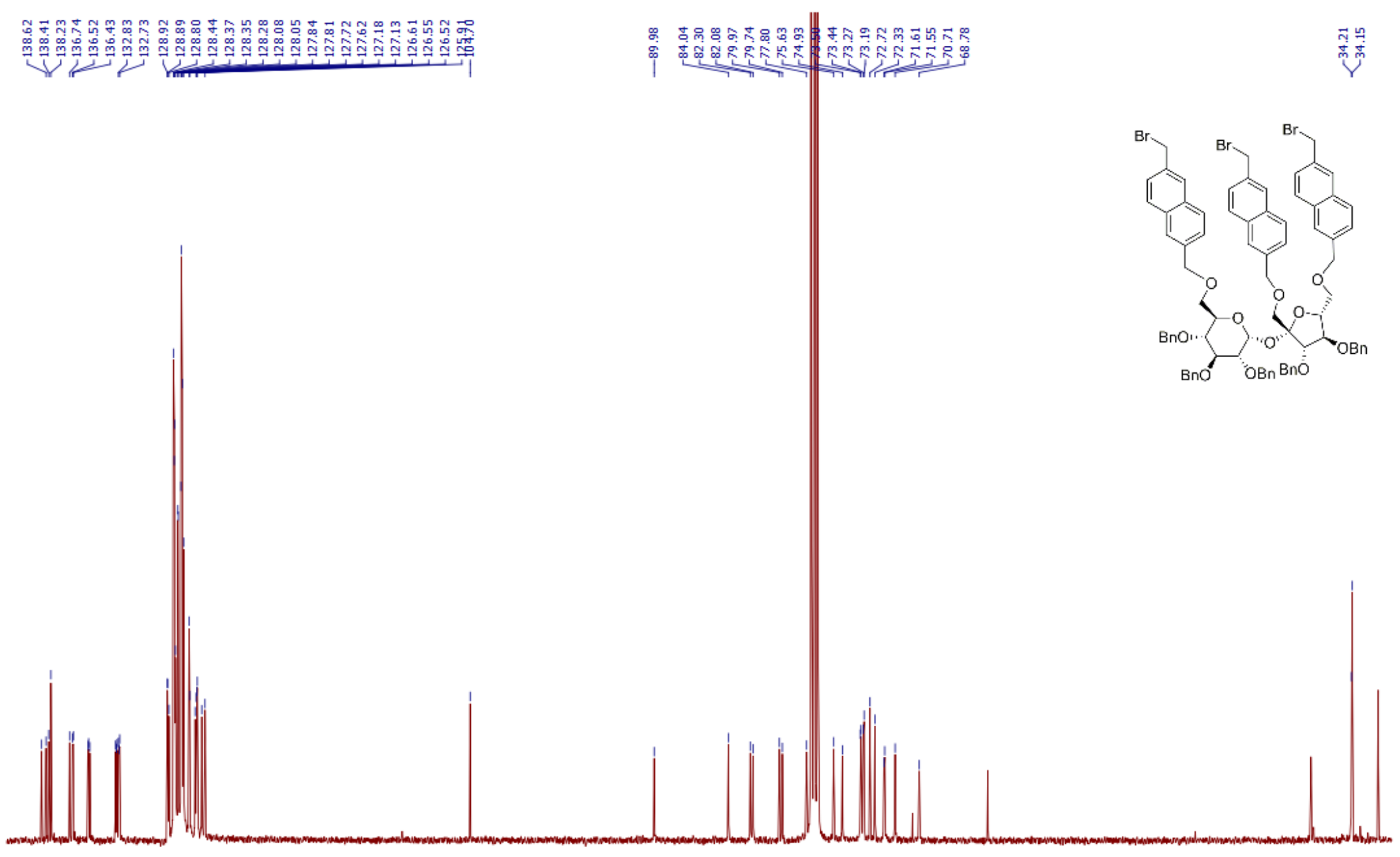

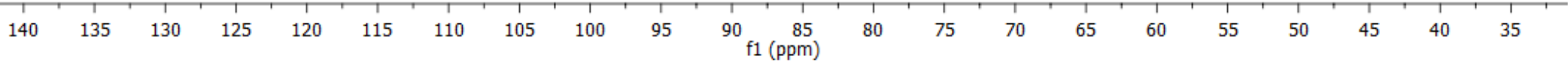

Figure S25. ${ }^{13} \mathrm{C}\left\{{ }^{1} \mathrm{H}\right\} \mathrm{NMR}\left(150 \mathrm{MHz}, \mathrm{CDCl}_{3}\right)$ spectrum of compound $\mathbf{3}$. 


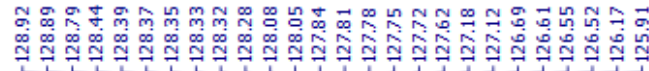

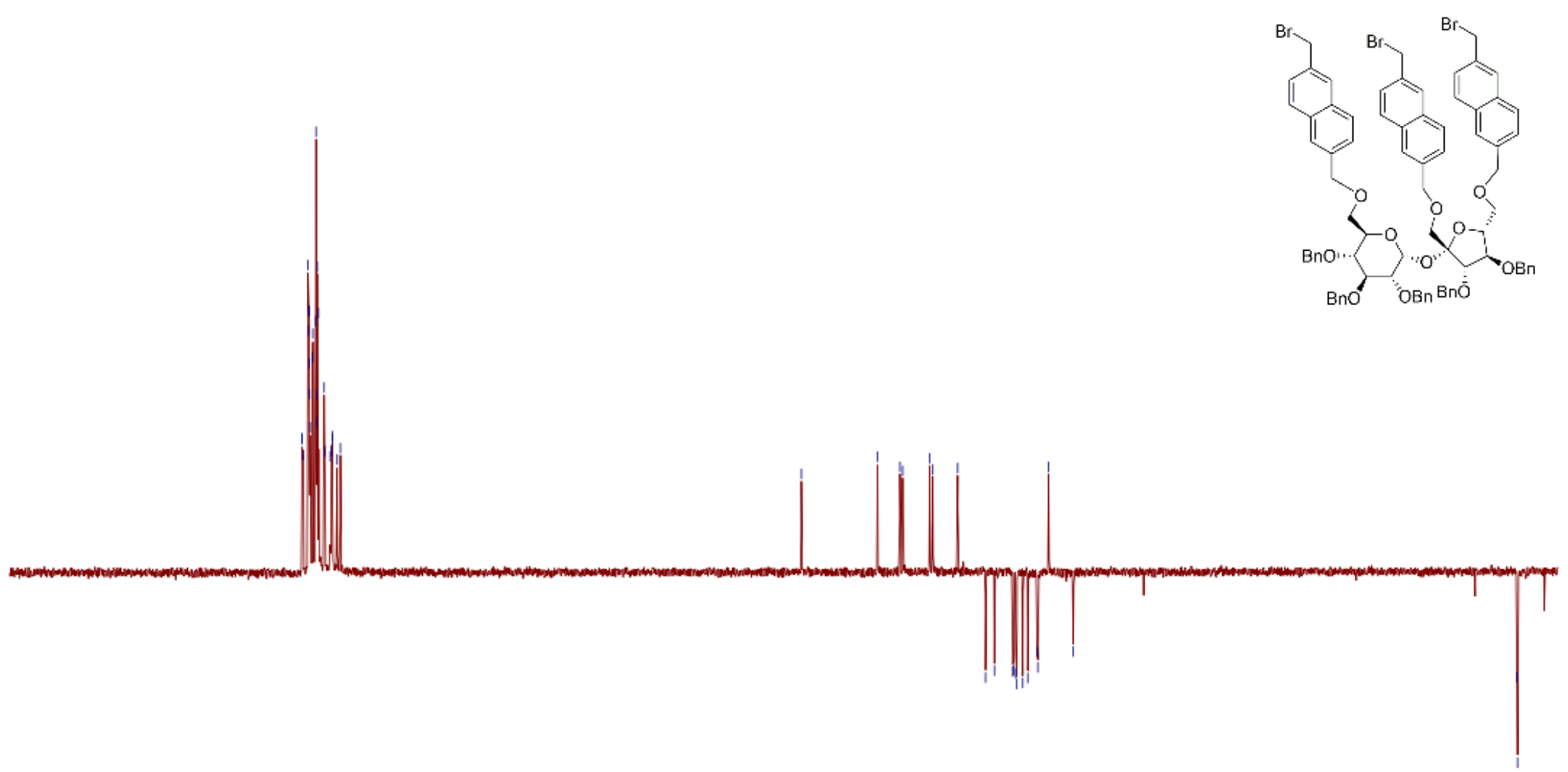

$\begin{array}{llll}150 & 145 & 140 & 135\end{array}$

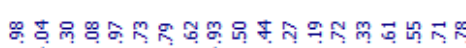

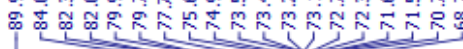

. 


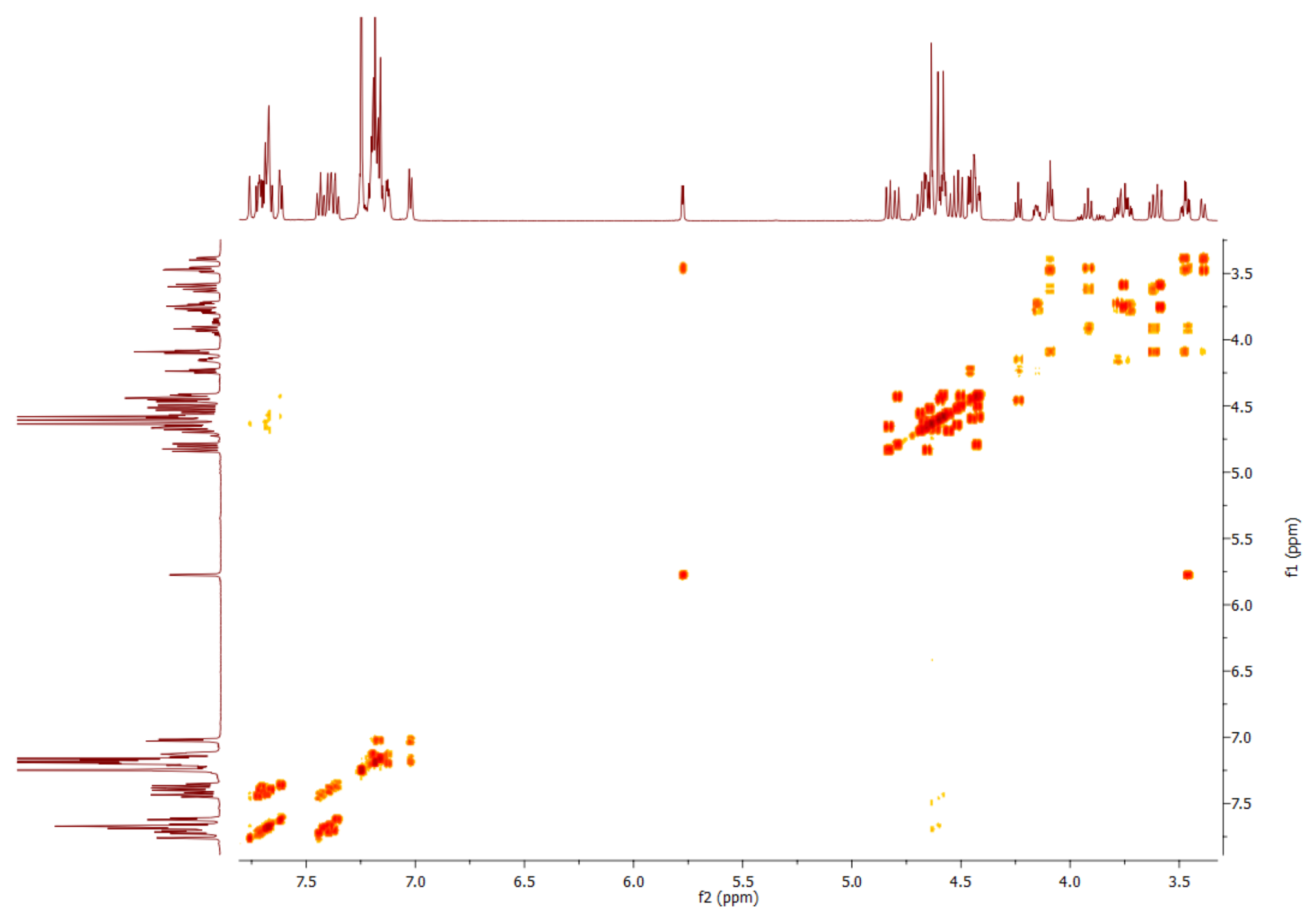

Figure S27. ${ }^{1} \mathrm{H}-{ }^{1} \mathrm{H} \operatorname{COSY}\left(600 \mathrm{MHz}, \mathrm{CDCl}_{3}\right)$ spectrum of compound 3. 


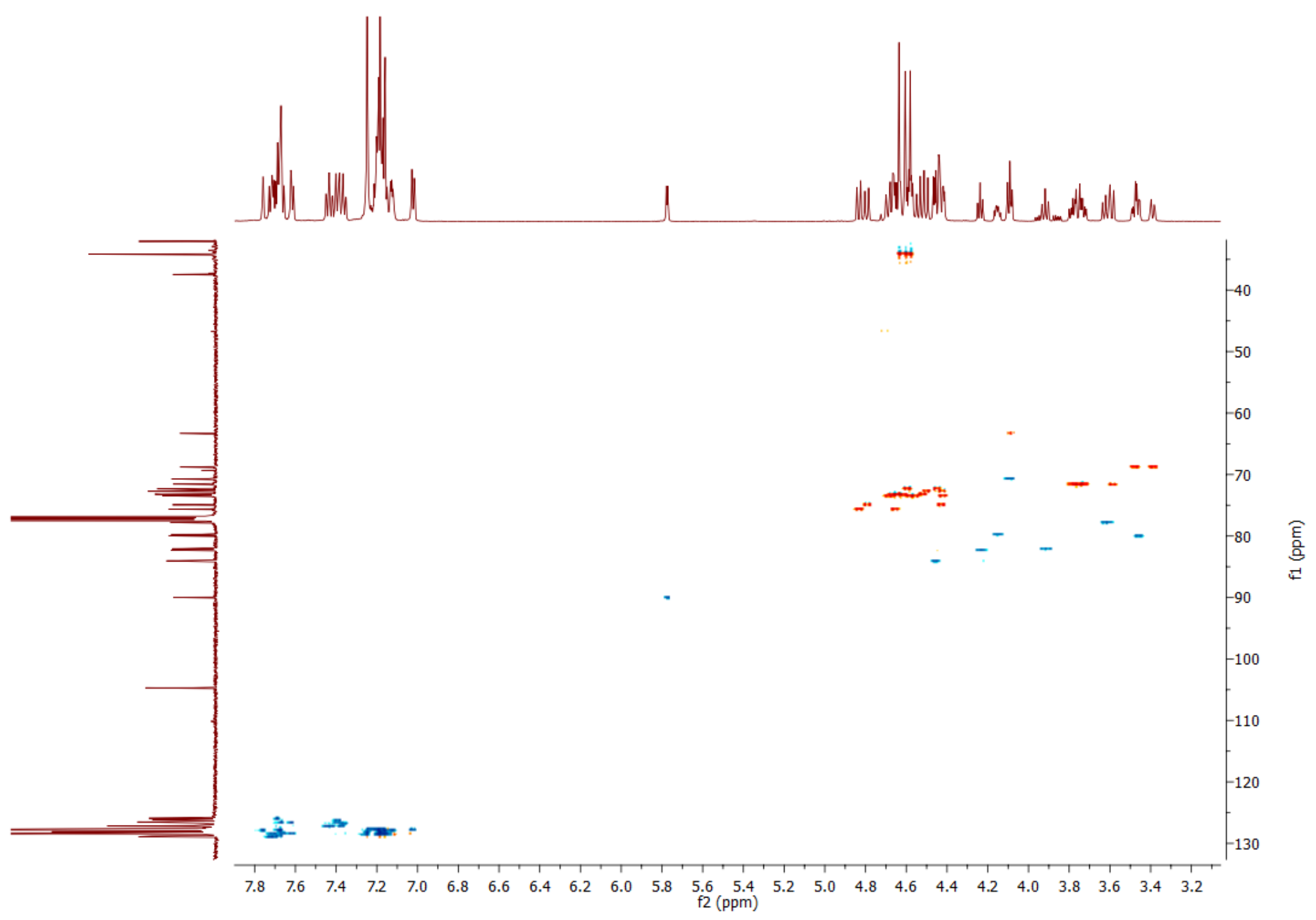

Figure S28. ${ }^{1} \mathrm{H}-{ }^{13} \mathrm{C}$ HSQC $\left(600 / 150 \mathrm{MHz}, \mathrm{CDCl}_{3}\right)$ spectrum of compound 3. 


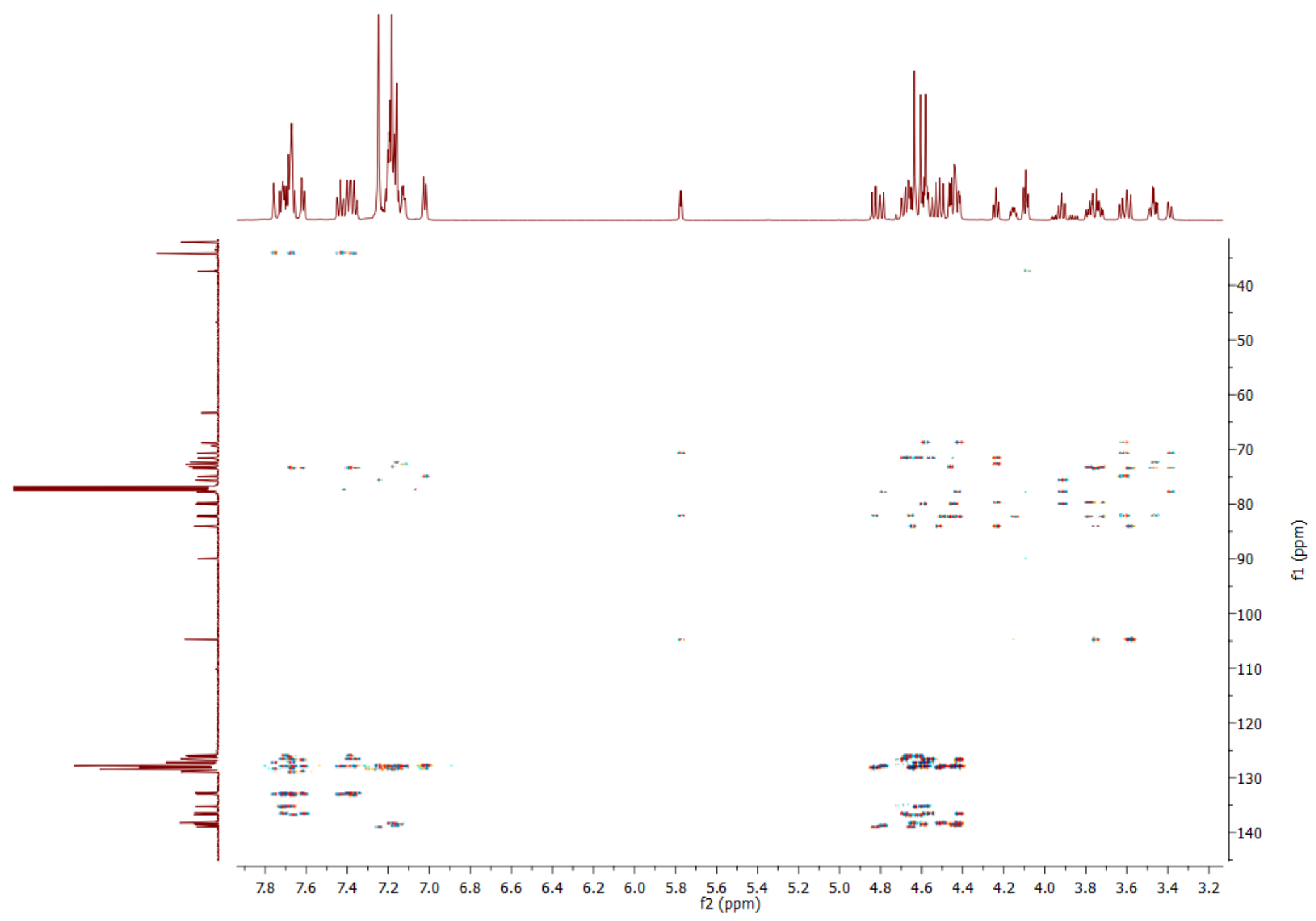

Figure S29. ${ }^{1} \mathrm{H}-{ }^{13} \mathrm{C} \mathrm{HMBC}\left(600 / 150 \mathrm{MHz}, \mathrm{CDCl}_{3}\right)$ spectrum of compound 3. 


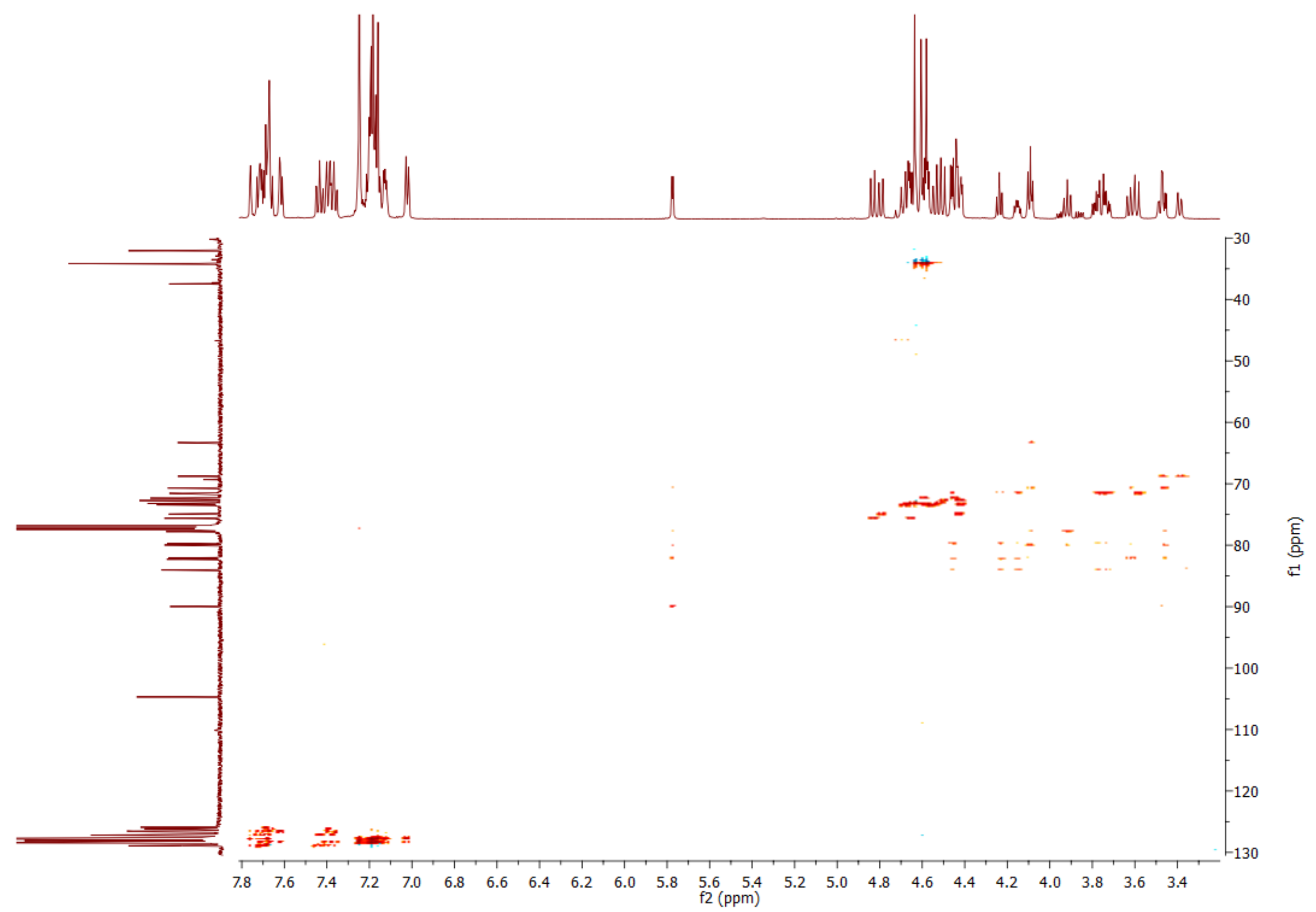

Figure S30. ${ }^{1} \mathrm{H}-{ }^{13} \mathrm{C}$ HSQC-TOCSY $\left(600 / 150 \mathrm{MHz}, \mathrm{CDCl}_{3}\right)$ spectrum of compound 3. 


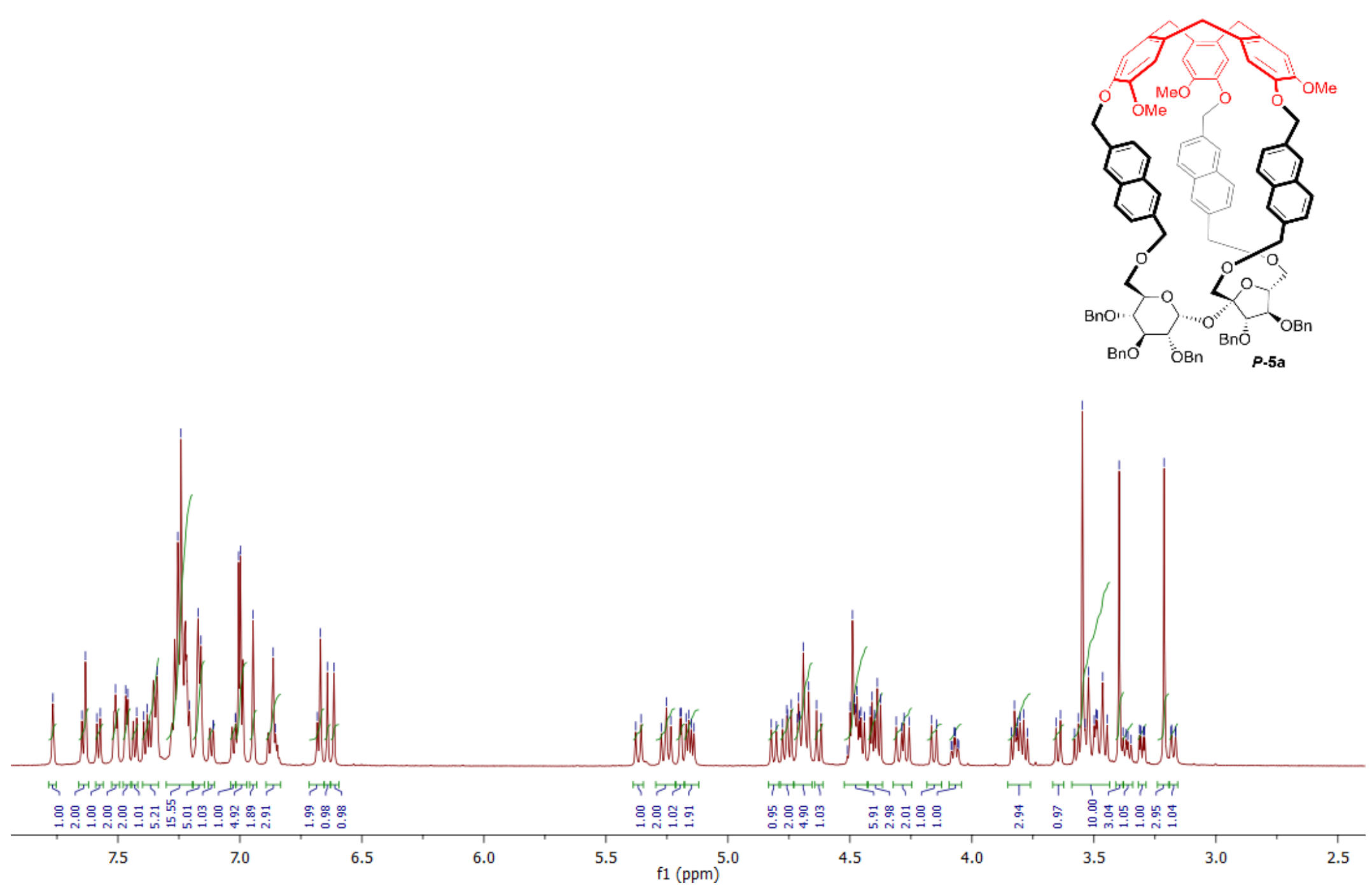

Figure S31. ${ }^{1} \mathrm{H} \mathrm{NMR}\left(600 \mathrm{MHz}, \mathrm{CDCl}_{3}\right)$ spectrum of compound $\boldsymbol{P}$-5a. 


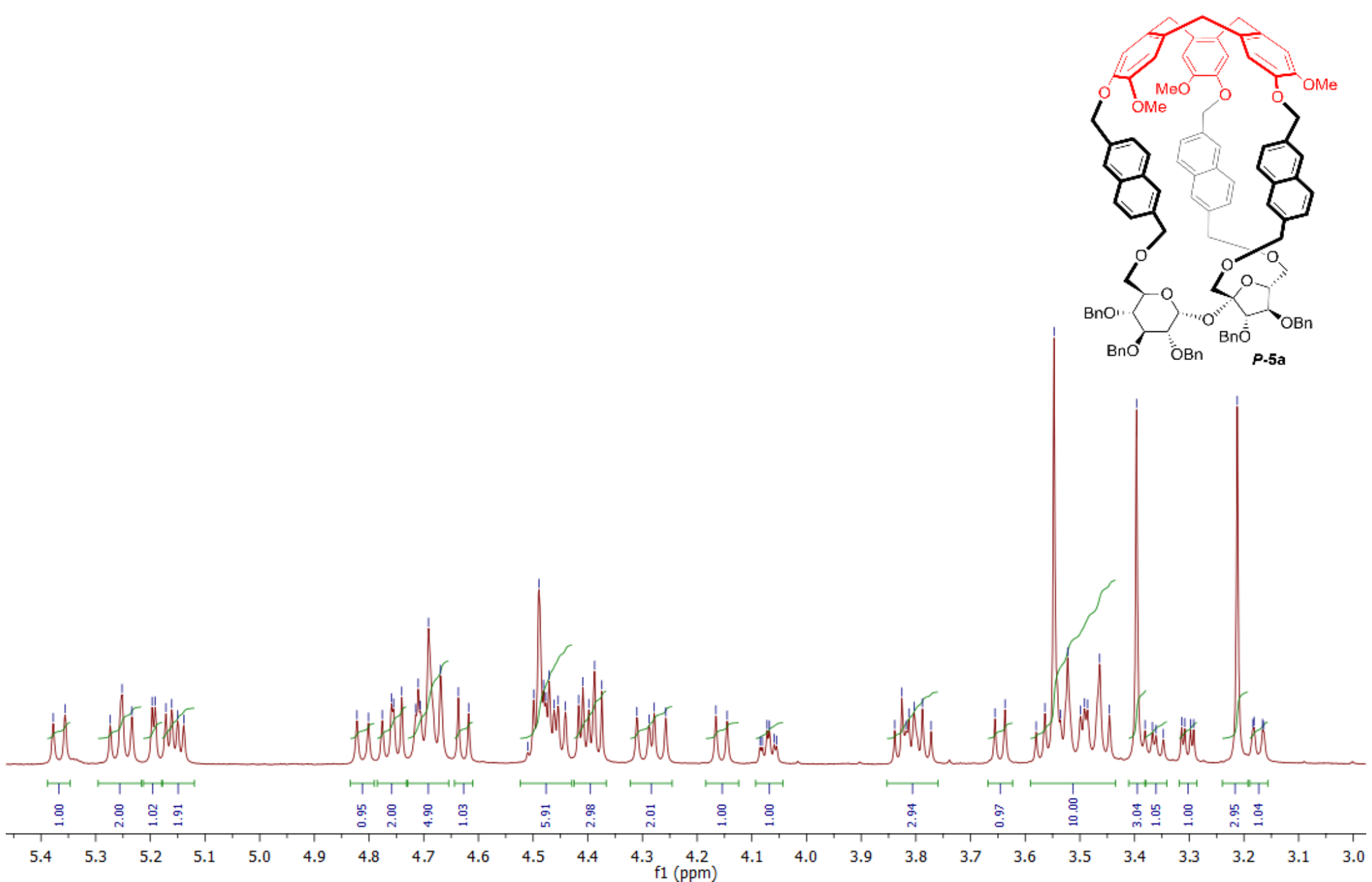

Figure S32. ${ }^{1} \mathrm{H}$ NMR $\left(600 \mathrm{MHz}, \mathrm{CDCl}_{3}\right)$ spectrum of compound $\boldsymbol{P}$-5a (aliphatic part). 

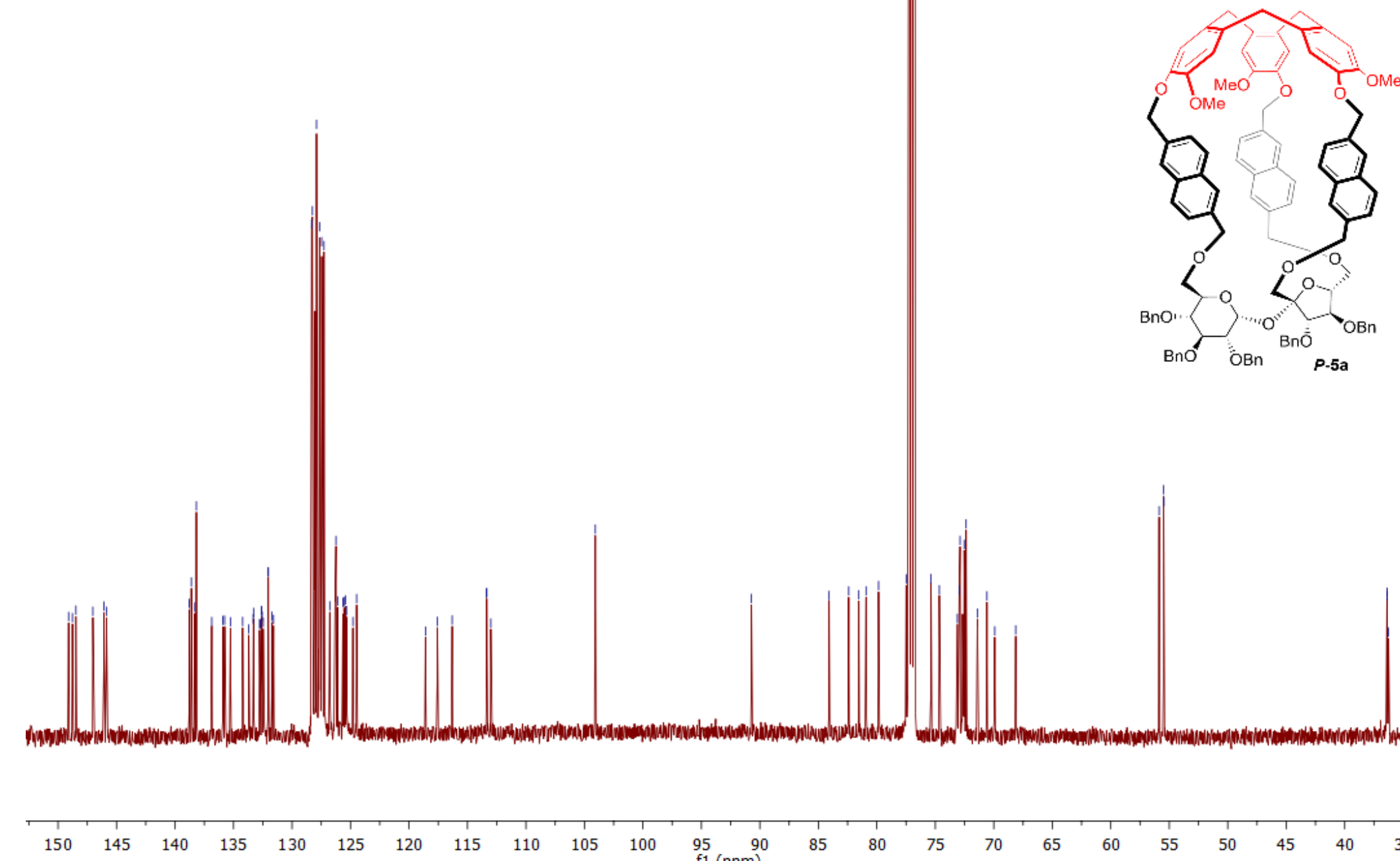
9590

Figure S33. ${ }^{13} \mathrm{C}\left\{{ }^{1} \mathrm{H}\right\}$ NMR $\left(150 \mathrm{MHz}, \mathrm{CDCl}_{3}\right)$ spectrum of compound $\boldsymbol{P}$-5a. 


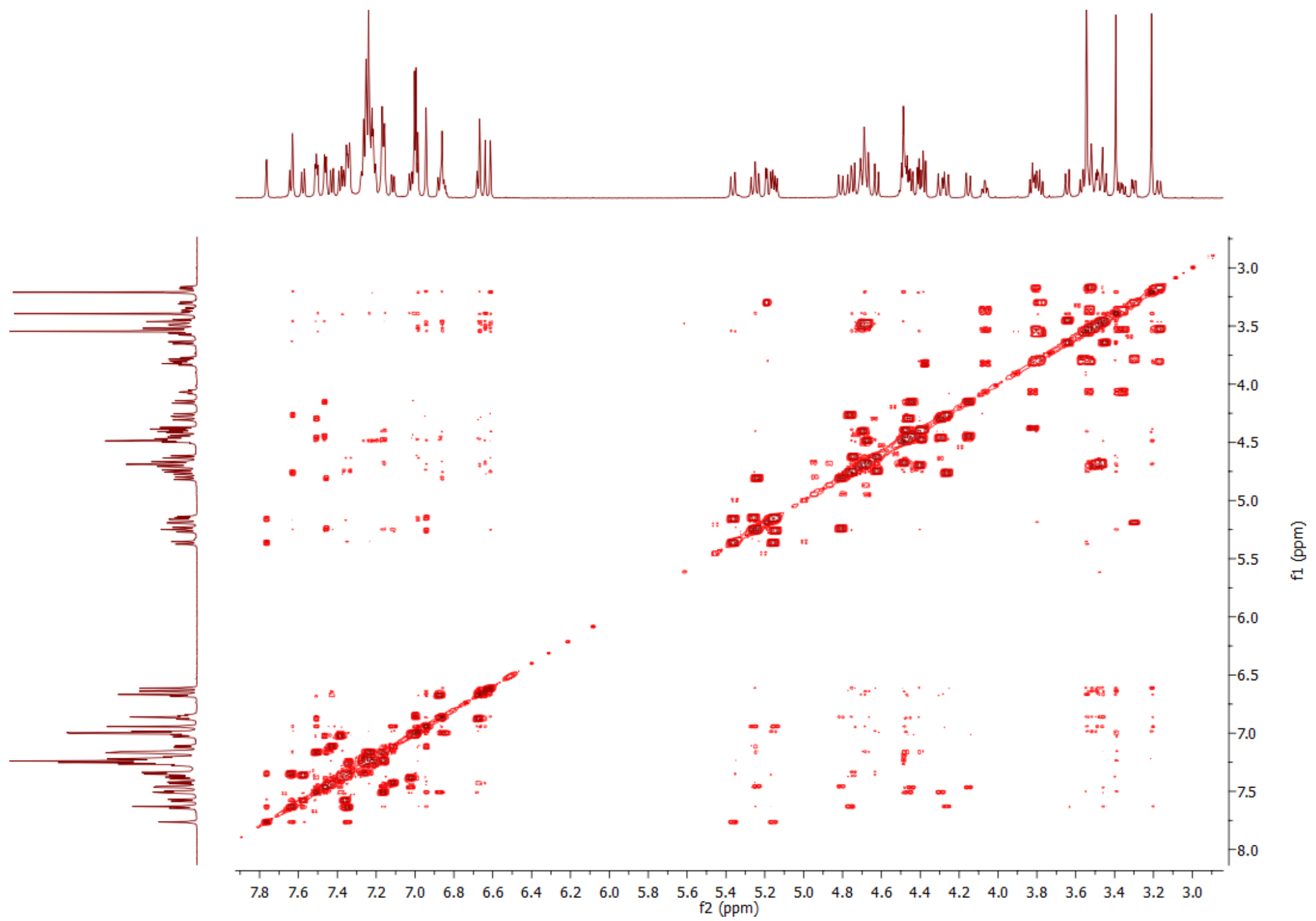

Figure S34. ${ }^{1} \mathrm{H}-{ }^{1} \mathrm{H}$ COSY $\left(600 \mathrm{MHz}, \mathrm{CDCl}_{3}\right)$ spectrum of compound $\mathbf{P}-\mathbf{5 a}$. 


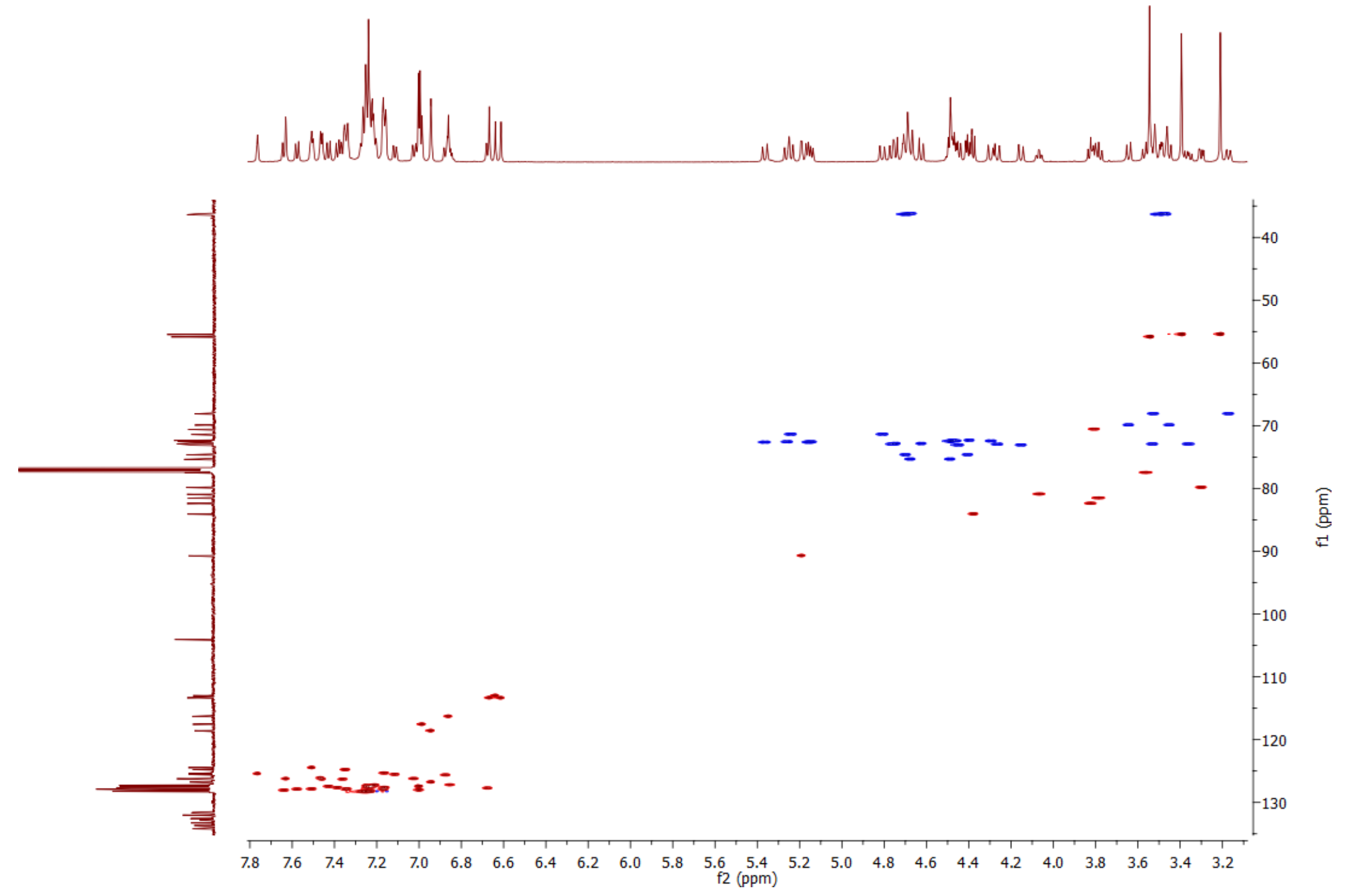

Figure S35. ${ }^{1} \mathrm{H}-{ }^{13} \mathrm{C} \mathrm{HSQC}\left(600 / 150 \mathrm{MHz}, \mathrm{CDCl}_{3}\right)$ spectrum of compound $\boldsymbol{P}-\mathbf{5 a}$. 


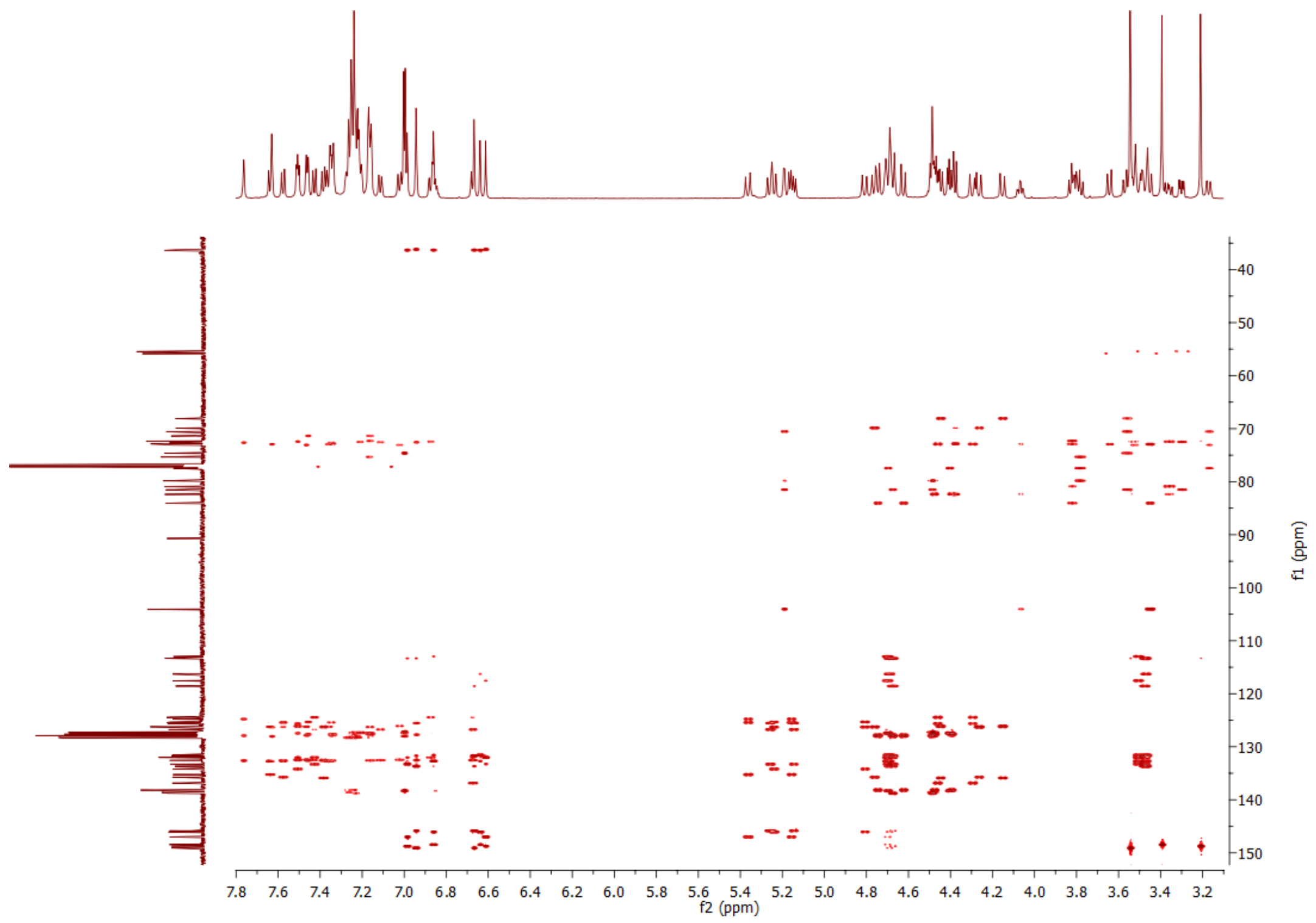

Figure S36. ${ }^{1} \mathrm{H}-{ }^{13} \mathrm{C} \mathrm{HMBC}\left(600 / 150 \mathrm{MHz}, \mathrm{CDCl}_{3}\right)$ spectrum of compound $\boldsymbol{P}-5$ a. 


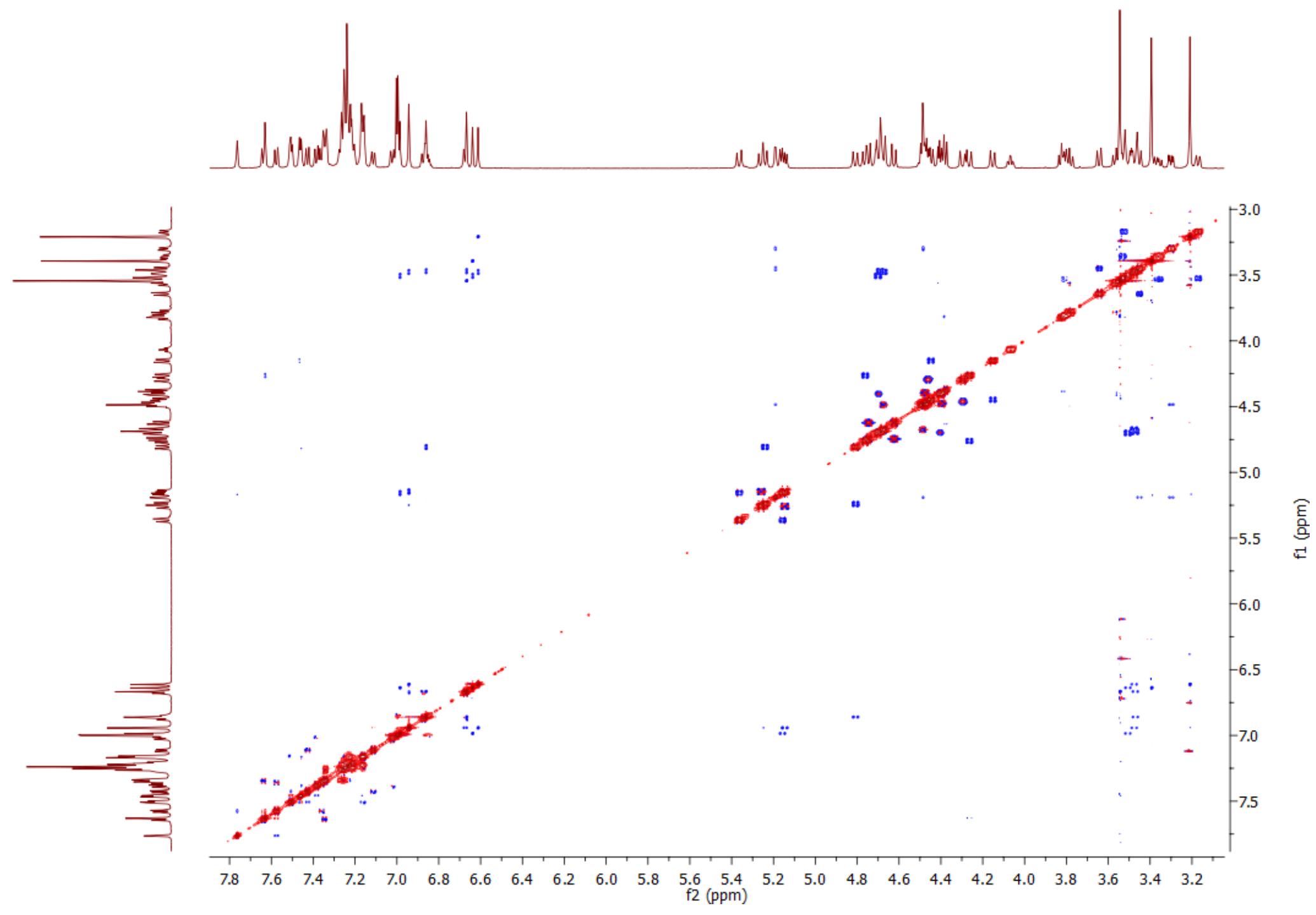

Figure S37. ${ }^{1} \mathrm{H}-{ }^{1} \mathrm{H}$ ROESY $\left(600 \mathrm{MHz}, \mathrm{CDCl}_{3}\right)$ spectrum of compound $\boldsymbol{P}-\mathbf{5 a}$. 


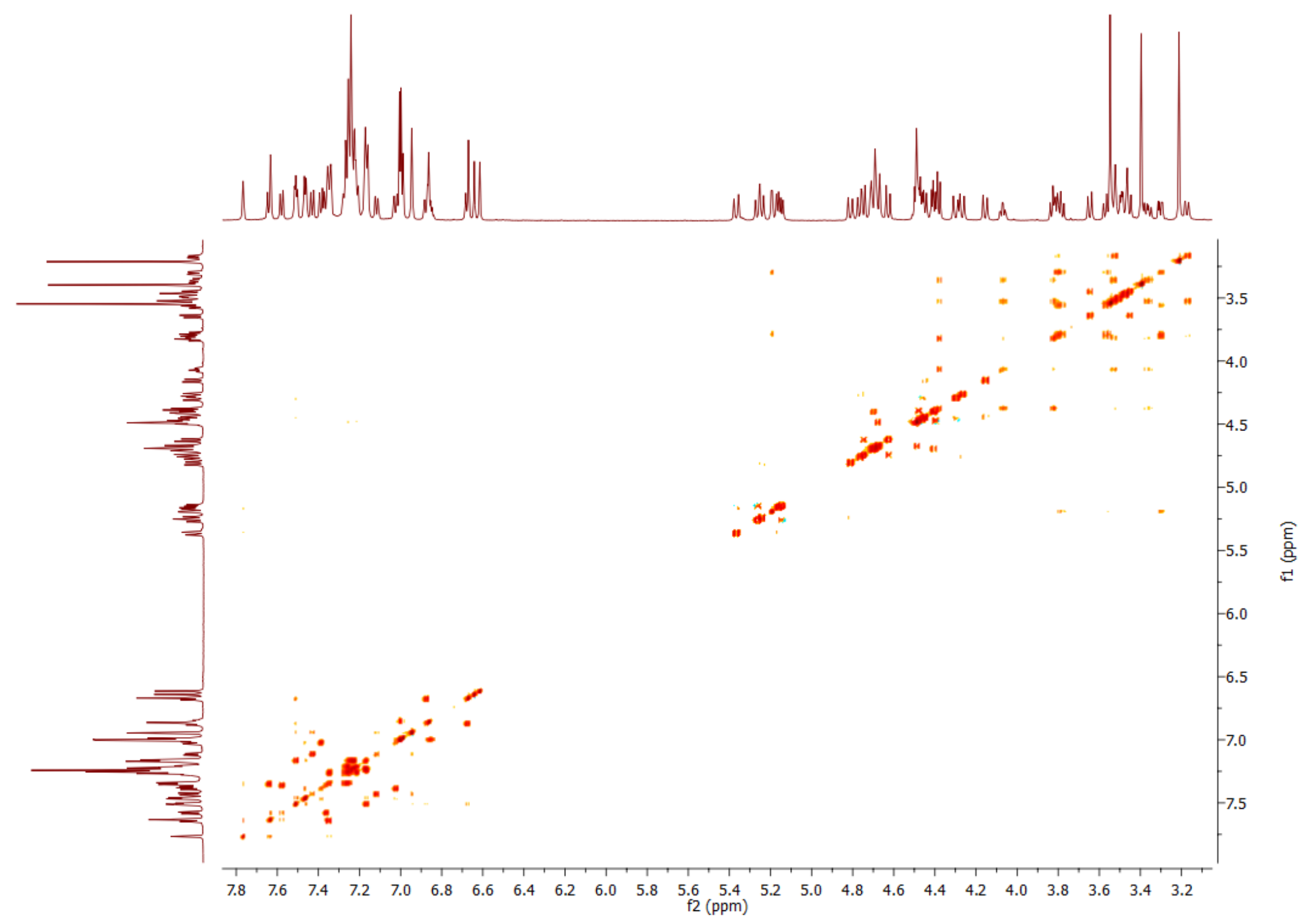

Figure S38. ${ }^{1} \mathrm{H}-{ }^{1} \mathrm{H}$ TOCSY $\left(600 \mathrm{MHz}, \mathrm{CDCl}_{3}\right)$ spectrum of compound $\boldsymbol{P}-\mathbf{5 a}$. 


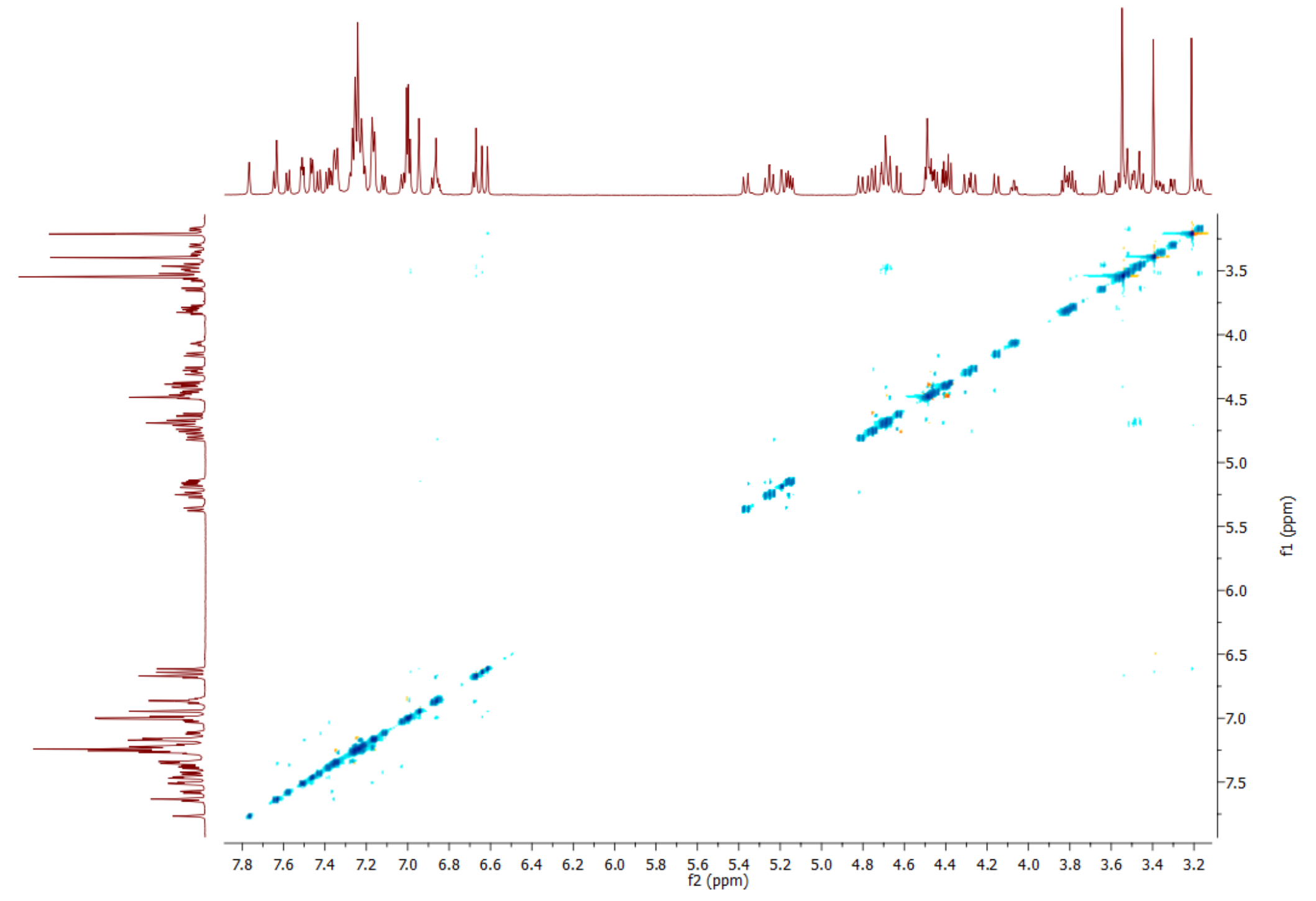

Figure S39. ${ }^{1} \mathrm{H}-{ }^{1} \mathrm{H}$ NOESY $\left(600 \mathrm{MHz}, \mathrm{CDCl}_{3}\right.$ ) spectrum of compound $\boldsymbol{P}-5 \mathbf{a}$. 


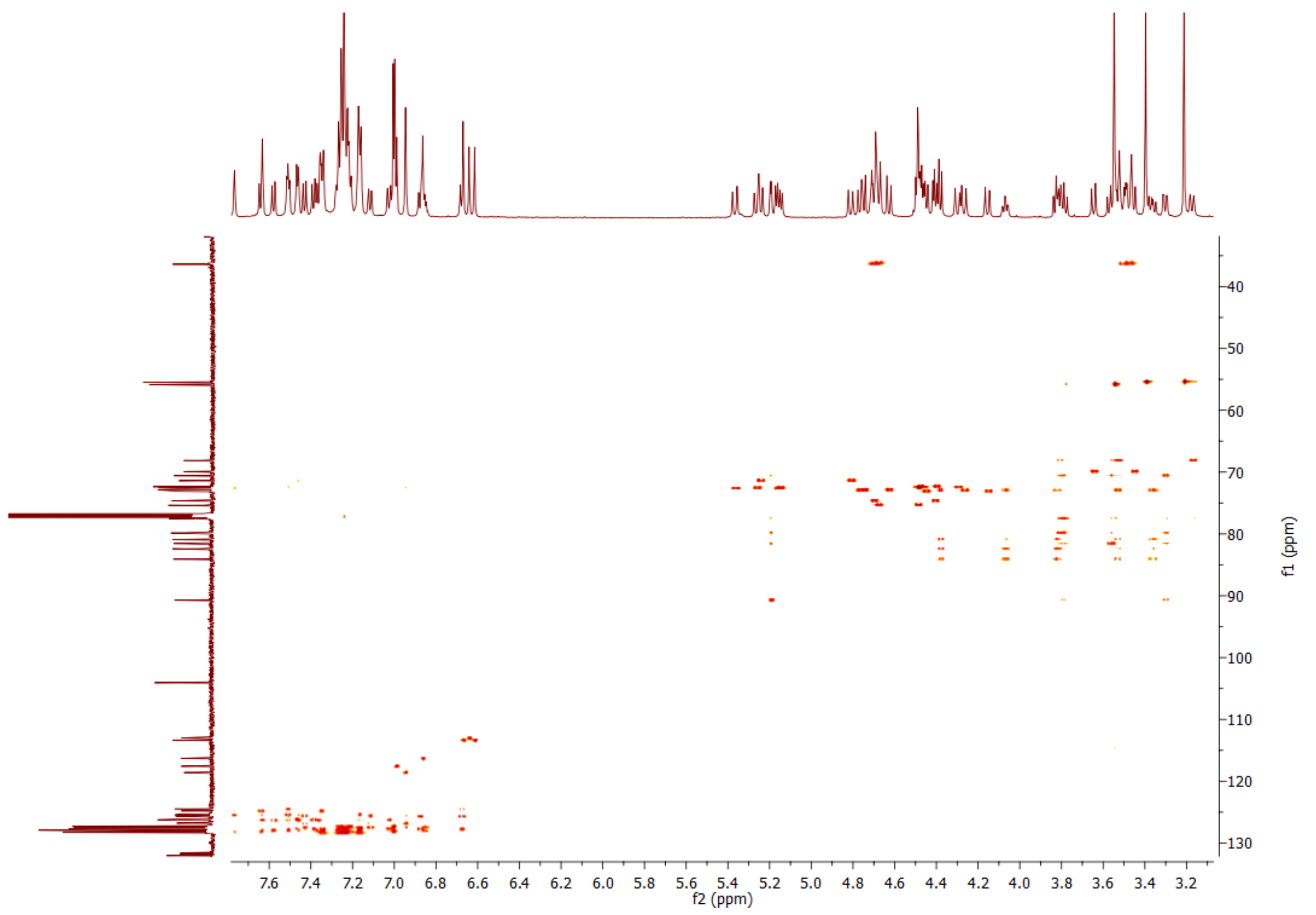

Figure S40. ${ }^{1} \mathrm{H}-{ }^{13} \mathrm{C}$ HSQC-TOCSY $\left(600 / 150 \mathrm{MHz}, \mathrm{CDCl}_{3}\right)$ spectrum of compound $\boldsymbol{P}-5 \mathbf{a}$. 


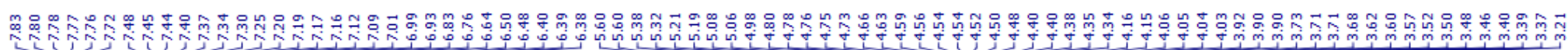

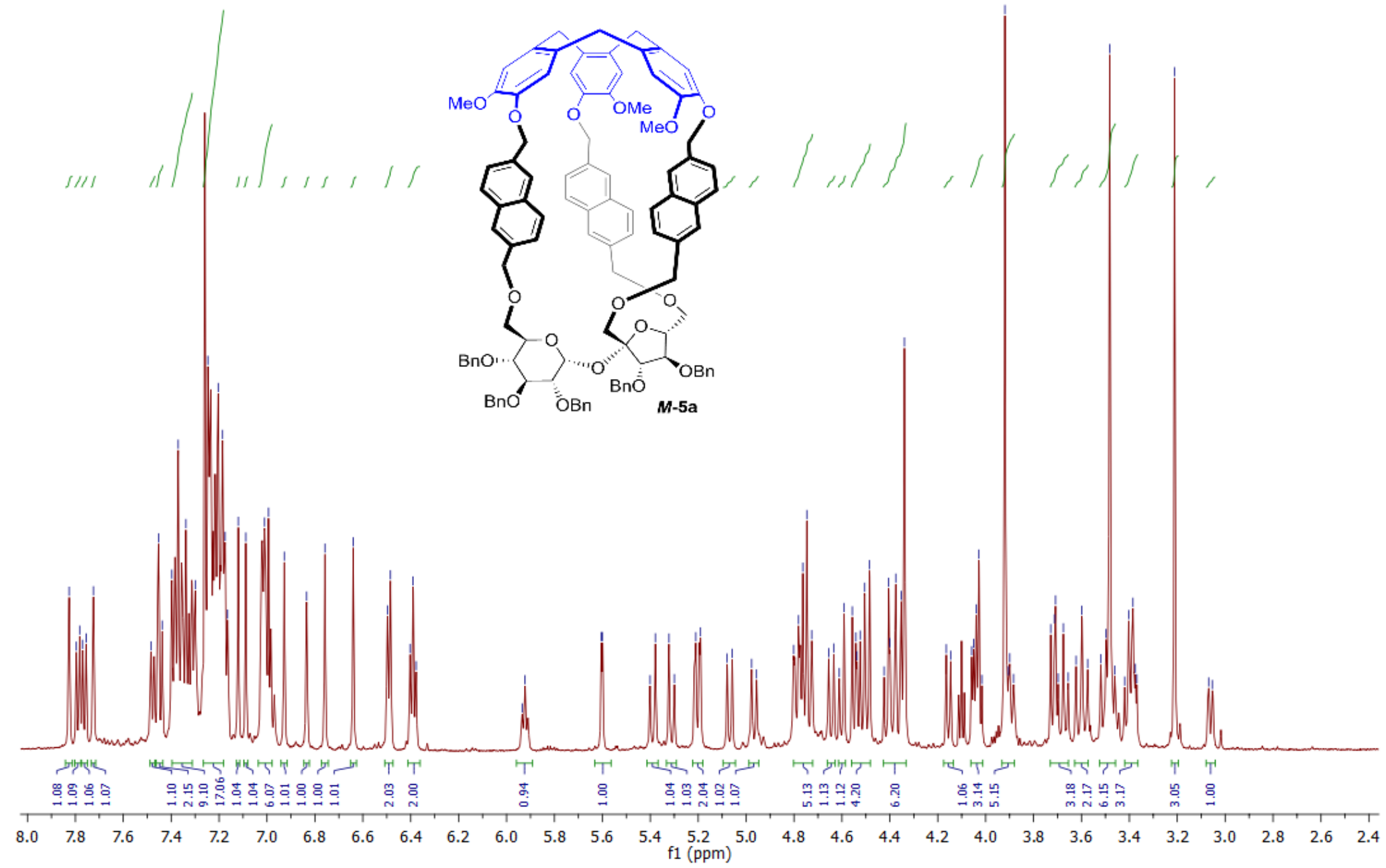

Figure S41. ${ }^{1} \mathrm{H}$ NMR $\left(600 \mathrm{MHz}, \mathrm{CDCl}_{3}\right)$ spectrum of compound $\boldsymbol{M}$-5a. 


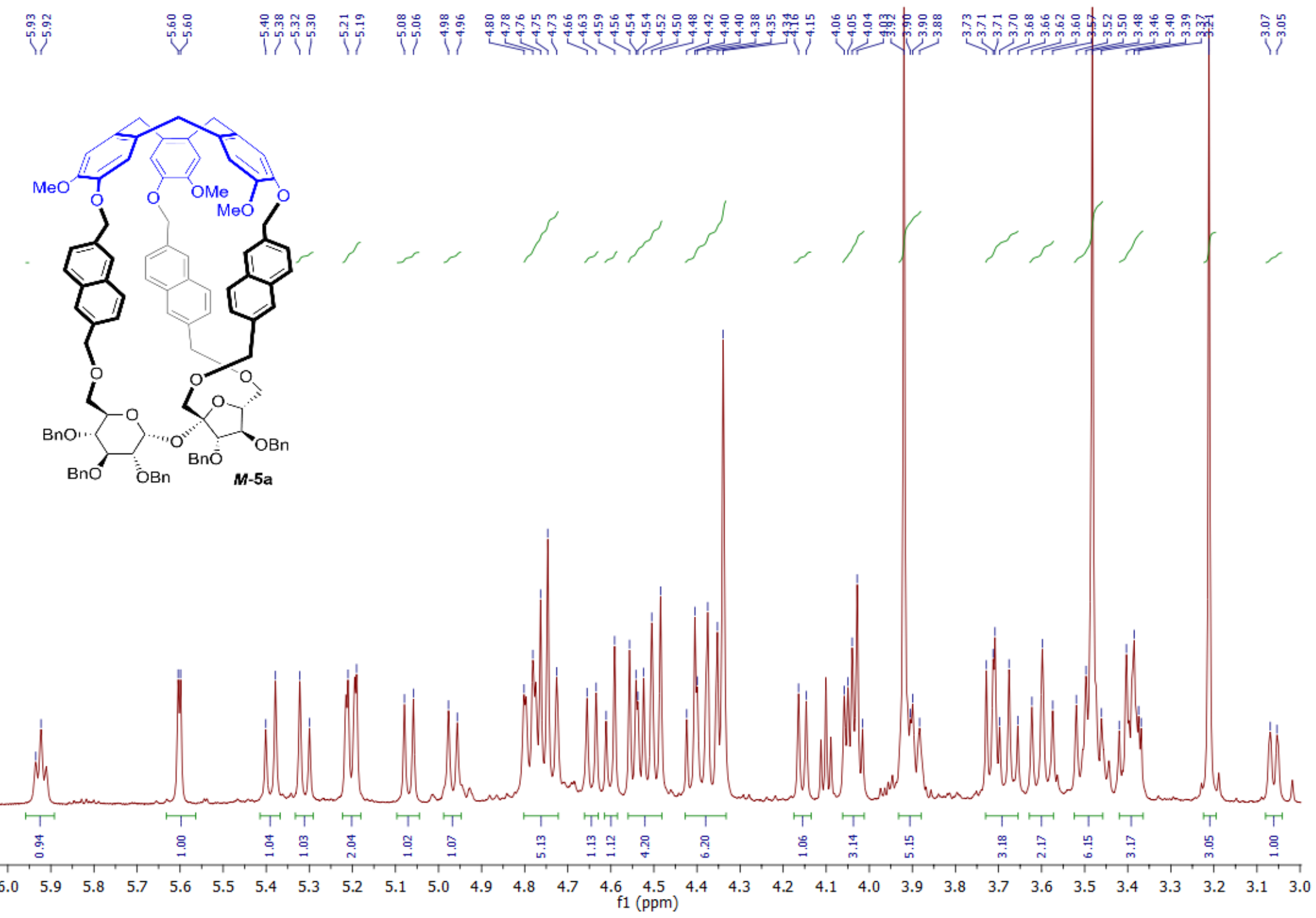

Figure S42. ${ }^{1} \mathrm{H}$ NMR $\left(600 \mathrm{MHz}, \mathrm{CDCl}_{3}\right)$ spectrum of compound $\boldsymbol{M}$-5a (aliphatic part). 

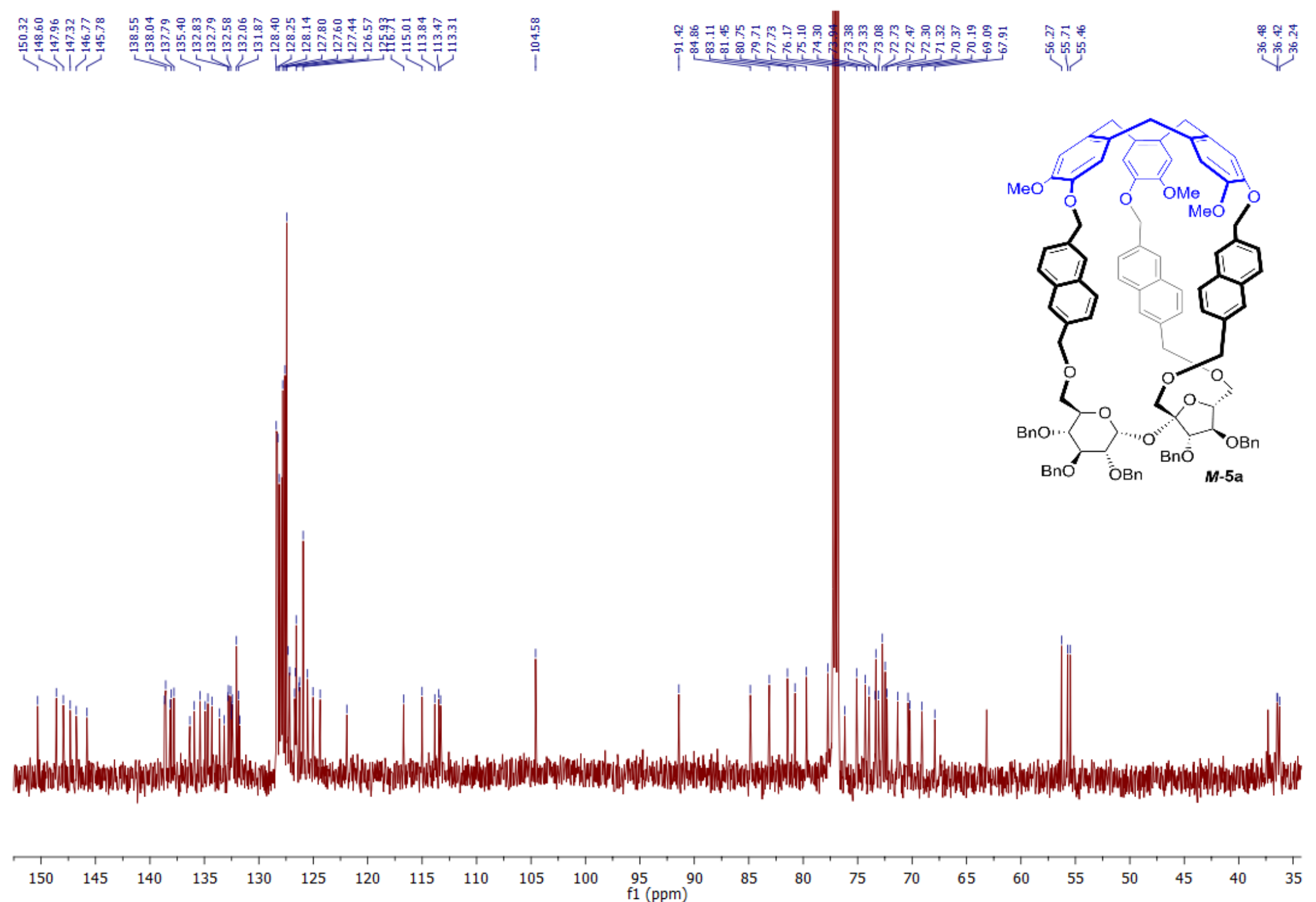

Figure S43. ${ }^{13} \mathrm{C}\left\{{ }^{1} \mathrm{H}\right\} \mathrm{NMR}\left(150 \mathrm{MHz}, \mathrm{CDCl}_{3}\right)$ spectrum of compound $\boldsymbol{M}-\mathbf{5 a}$. 


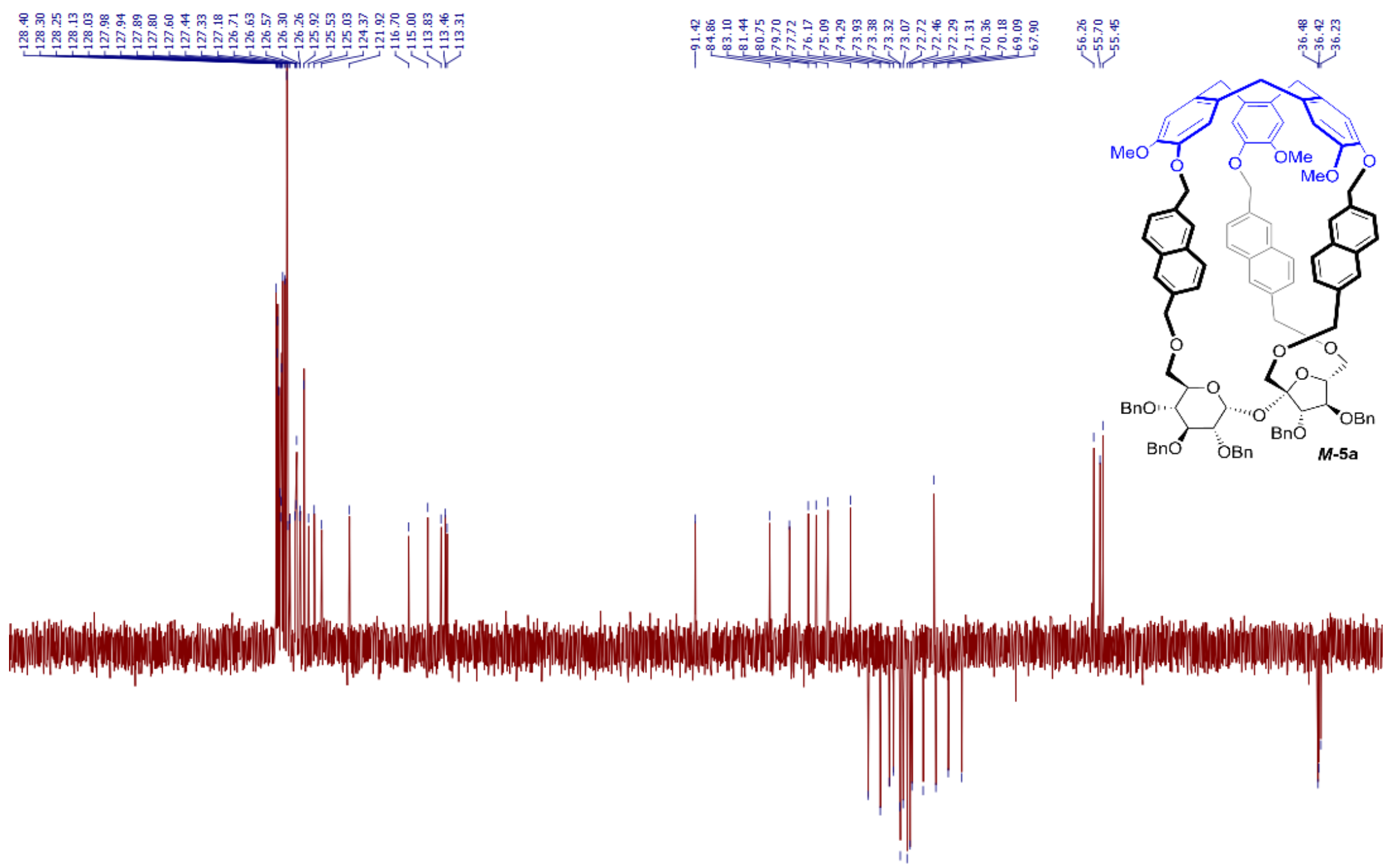

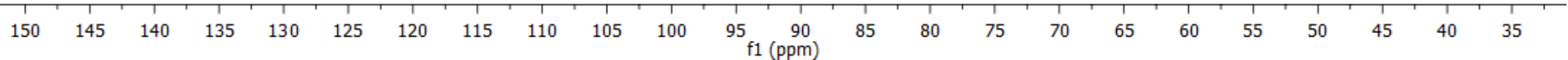

Figure S44. ${ }^{13} \mathrm{C}\left\{{ }^{1} \mathrm{H}\right\}$ DEPT (150 $\left.\mathrm{MHz}, \mathrm{CDCl}_{3}\right)$ spectrum of compound $\boldsymbol{M}-\mathbf{5 a}$. 


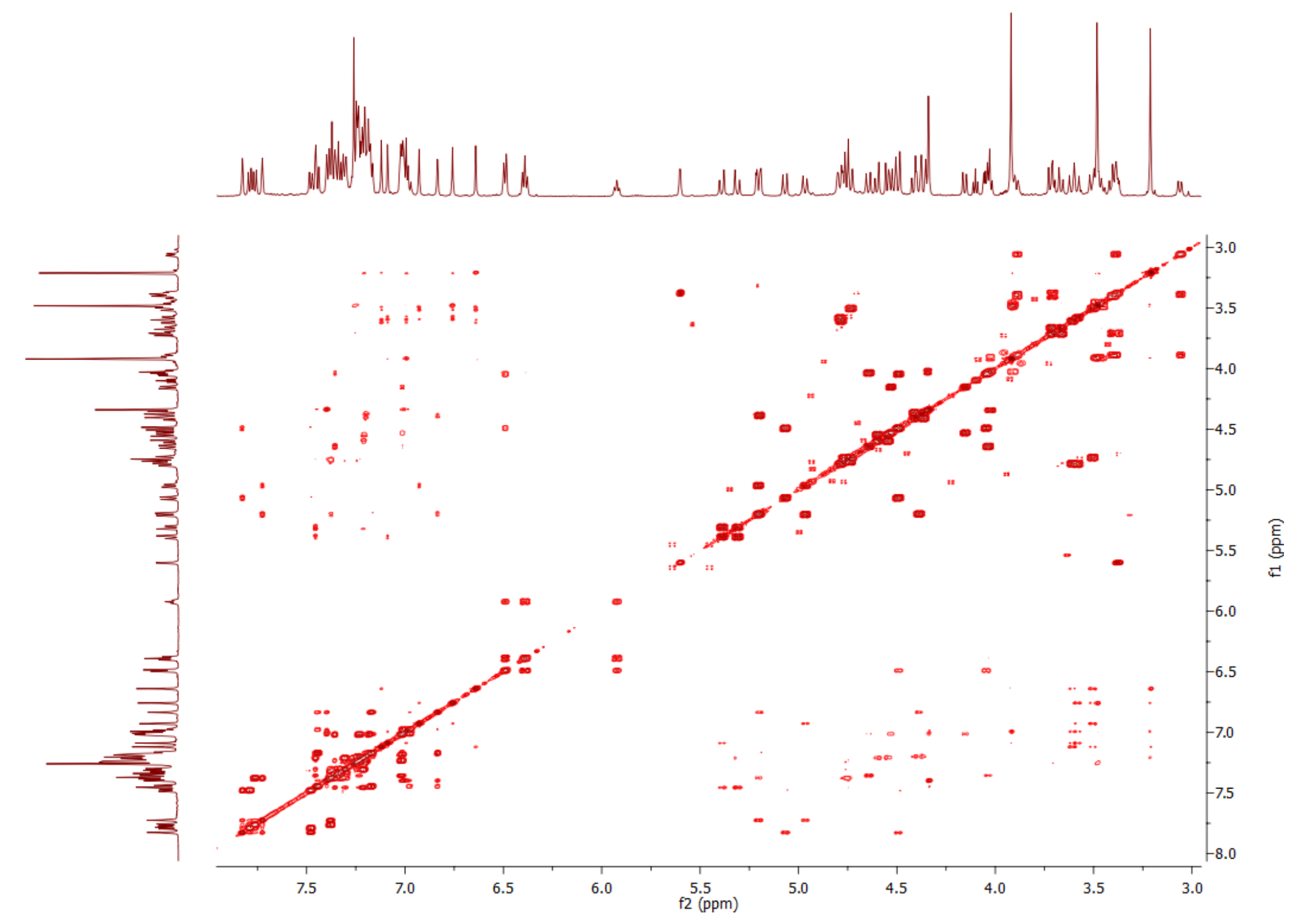

Figure S45. ${ }^{1} \mathrm{H}-{ }^{1} \mathrm{H}$ COSY $\left(600 \mathrm{MHz}, \mathrm{CDCl}_{3}\right)$ spectrum of compound $\boldsymbol{M}$-5a. 


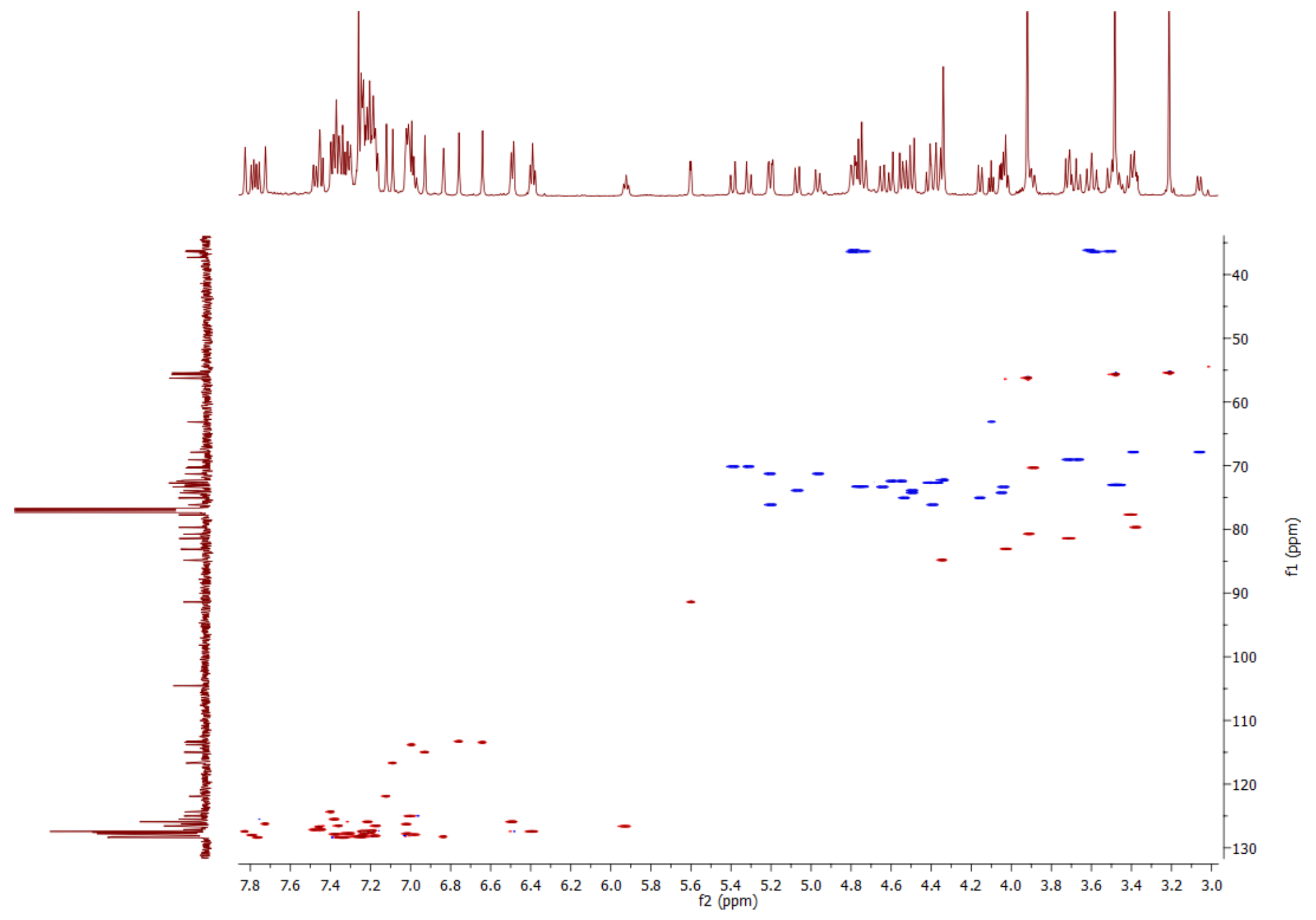

Figure S46. ${ }^{1} \mathrm{H}^{13}{ }^{13} \mathrm{HSQC}\left(600 / 150 \mathrm{MHz}, \mathrm{CDCl}_{3}\right)$ spectrum of compound $\boldsymbol{M}-5 \mathbf{a}$. 


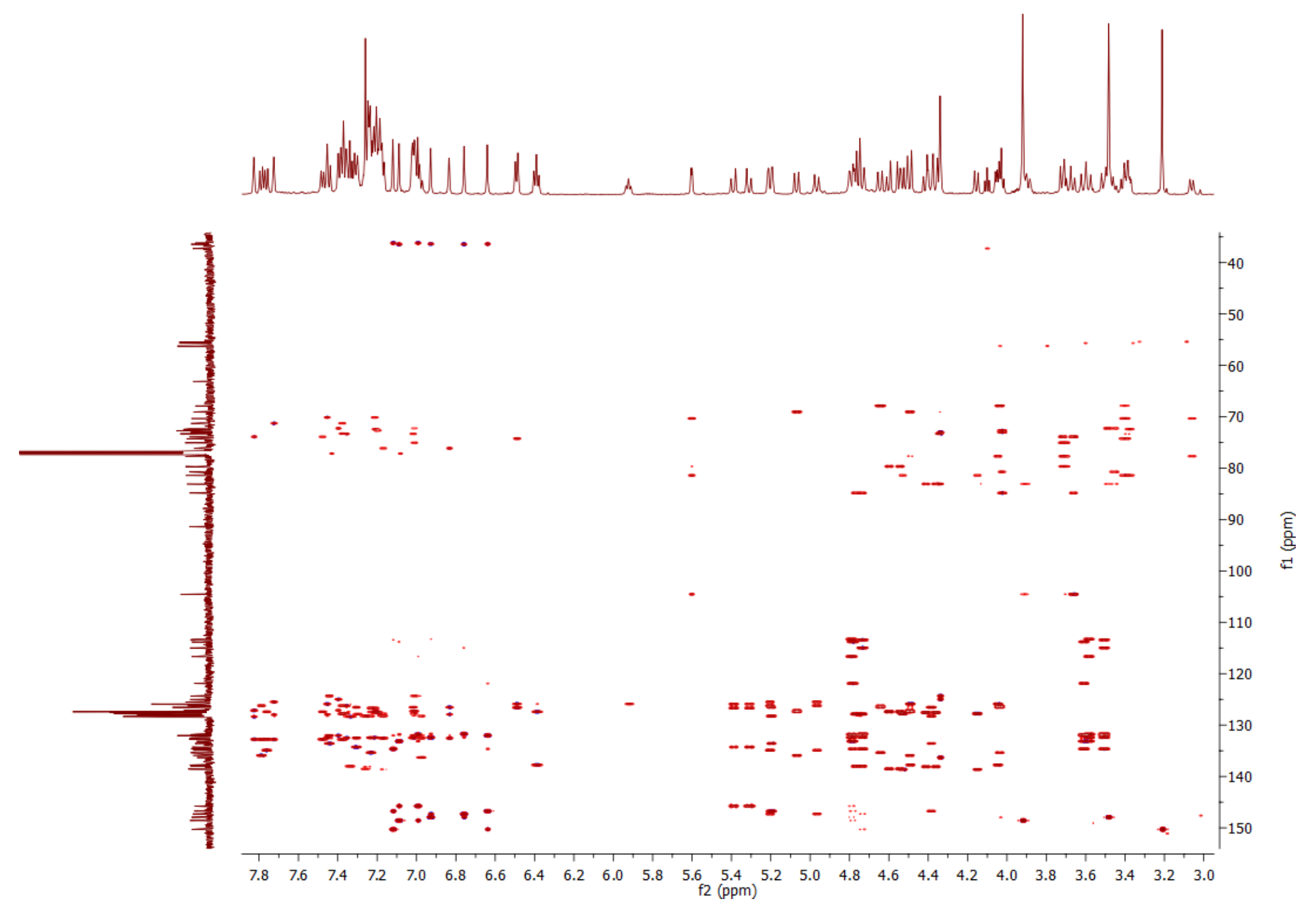

Figure S47. ${ }^{1} \mathrm{H}-{ }^{13} \mathrm{C} \mathrm{HMBC}\left(600 / 150 \mathrm{MHz}, \mathrm{CDCl}_{3}\right)$ spectrum of compound $\boldsymbol{M}-5 \mathbf{a}$. 


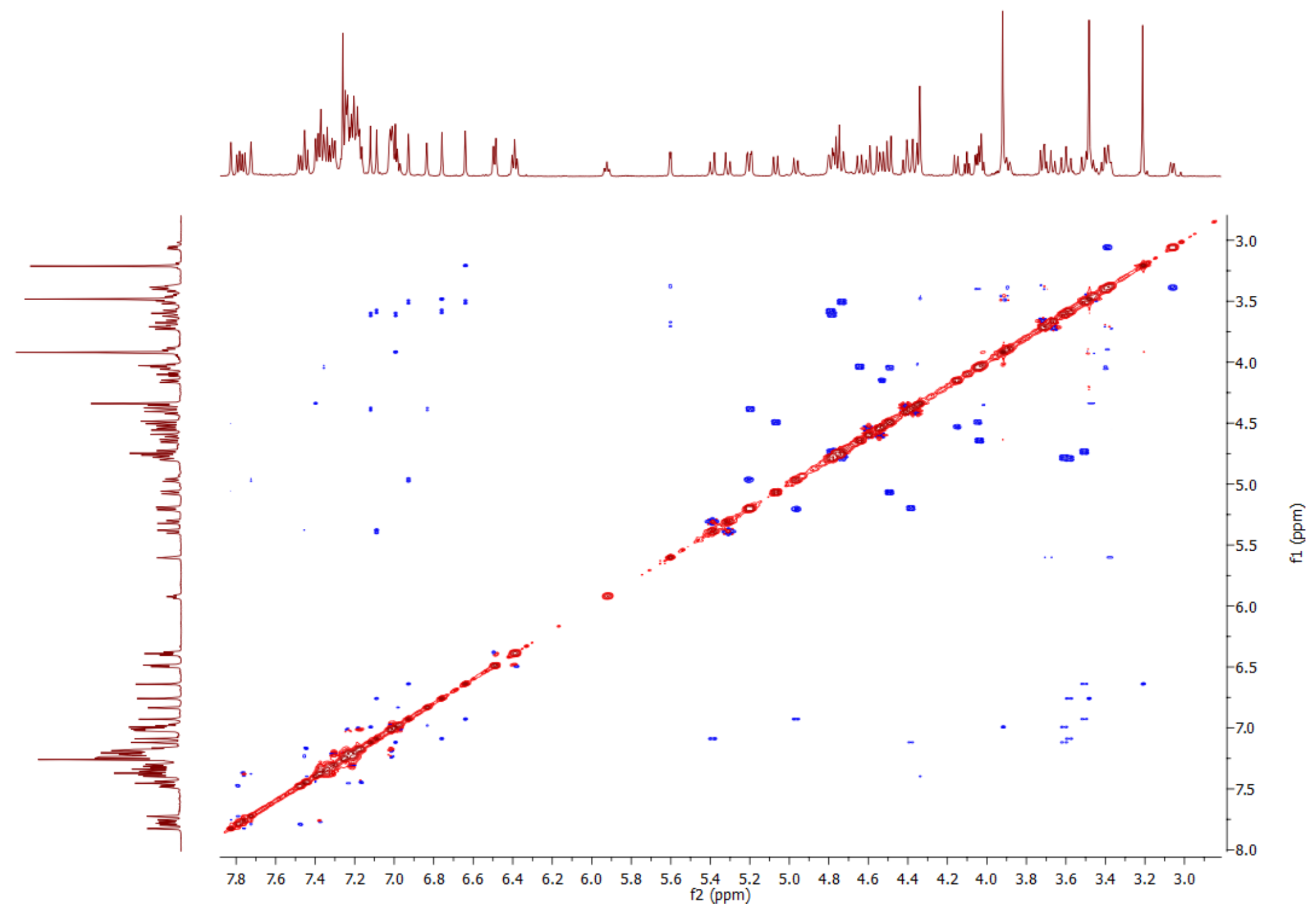

Figure S48. ${ }^{1} \mathrm{H}-{ }^{1} \mathrm{H}$ ROESY (600 MHz, $\mathrm{CDCl}_{3}$ ) spectrum of compound $\boldsymbol{M}$-5a. 


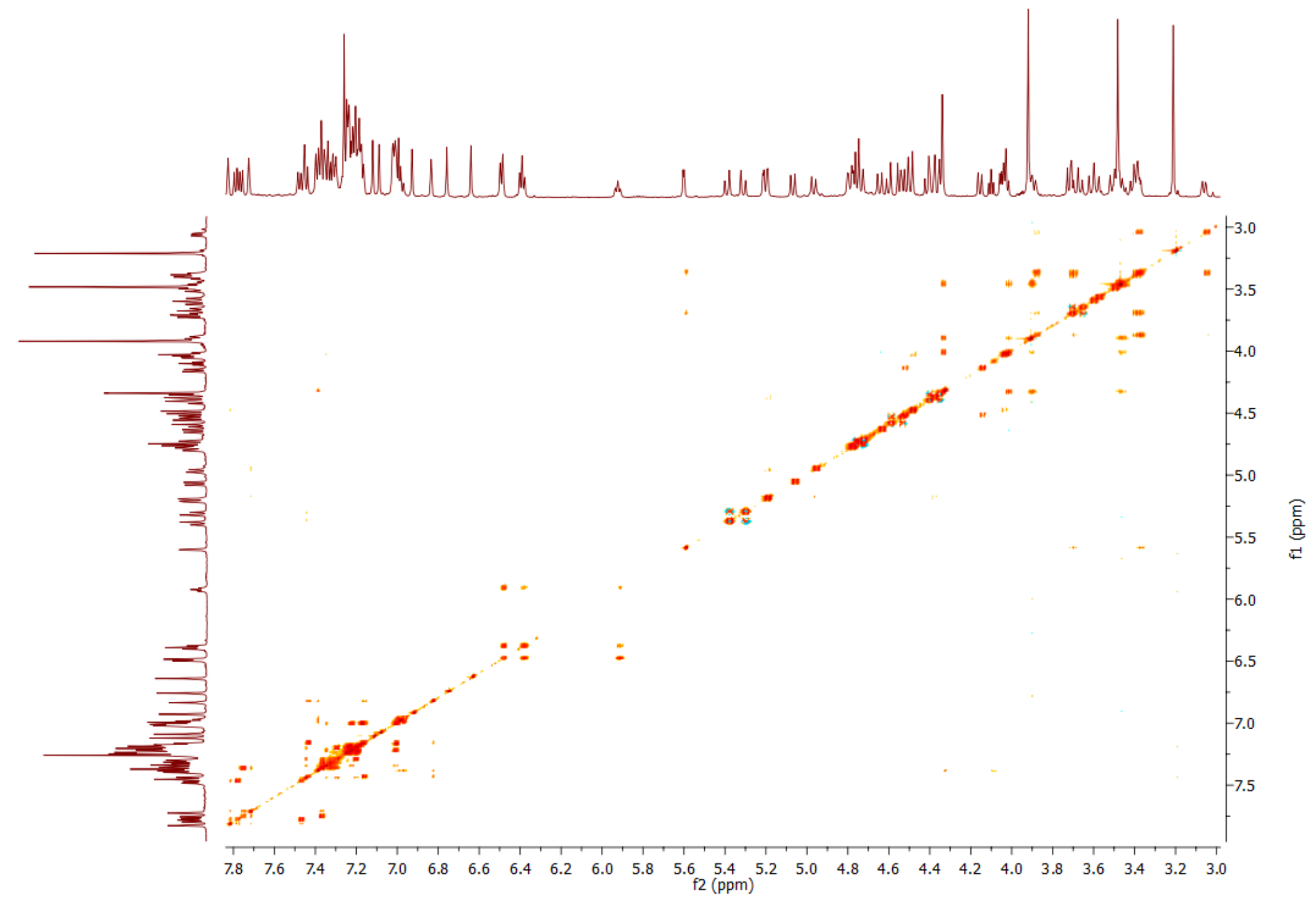

Figure S49. ${ }^{1} \mathrm{H}-{ }^{1} \mathrm{H}$ TOCSY $\left(600 \mathrm{MHz}, \mathrm{CDCl}_{3}\right.$ ) spectrum of compound $\boldsymbol{M}-\mathbf{5 a}$. 


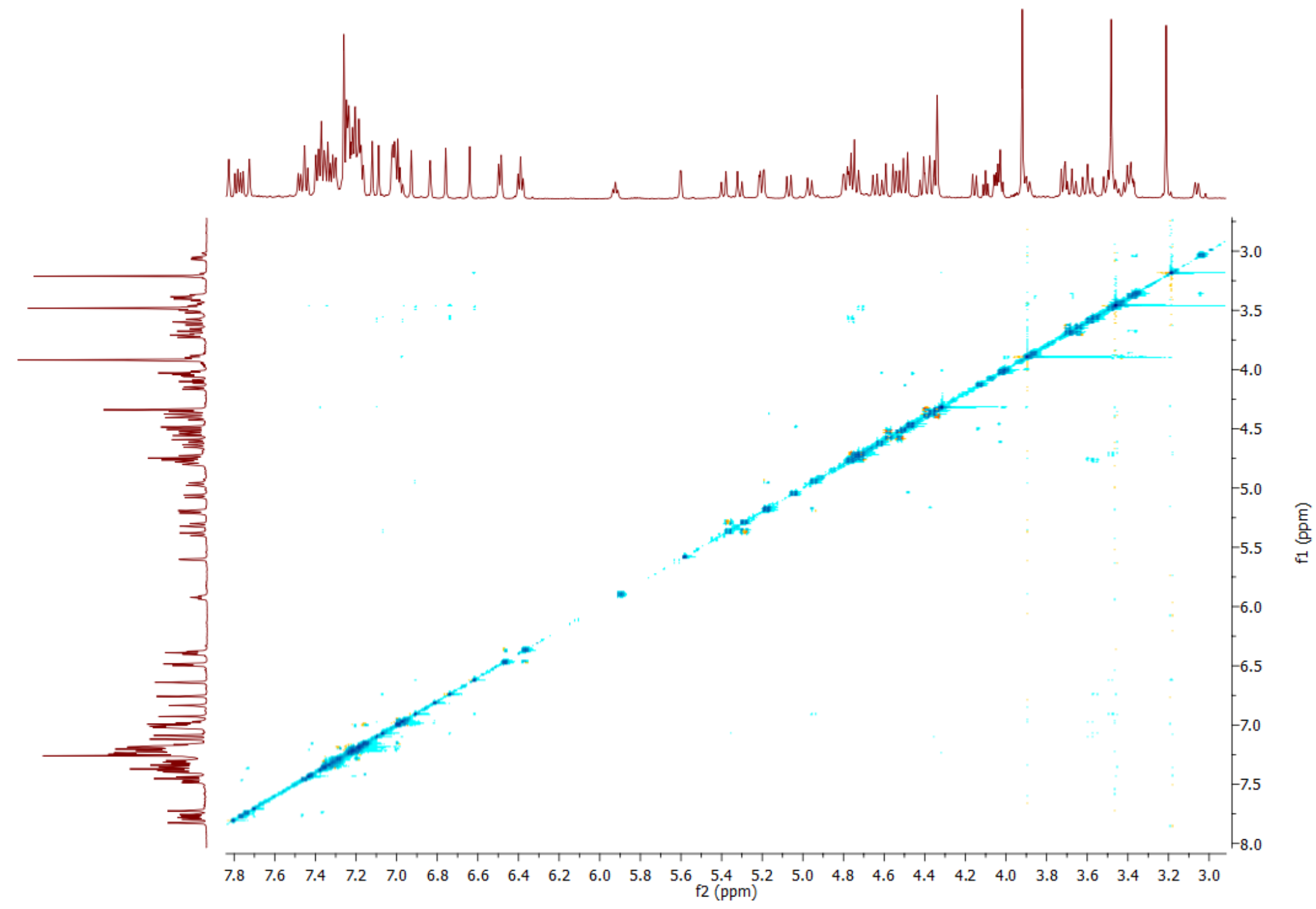

Figure S50. ${ }^{1} \mathrm{H}-{ }^{1} \mathrm{H}$ NOESY (600 MHz, $\mathrm{CDCl}_{3}$ ) spectrum of compound $\boldsymbol{M}$-5a. 


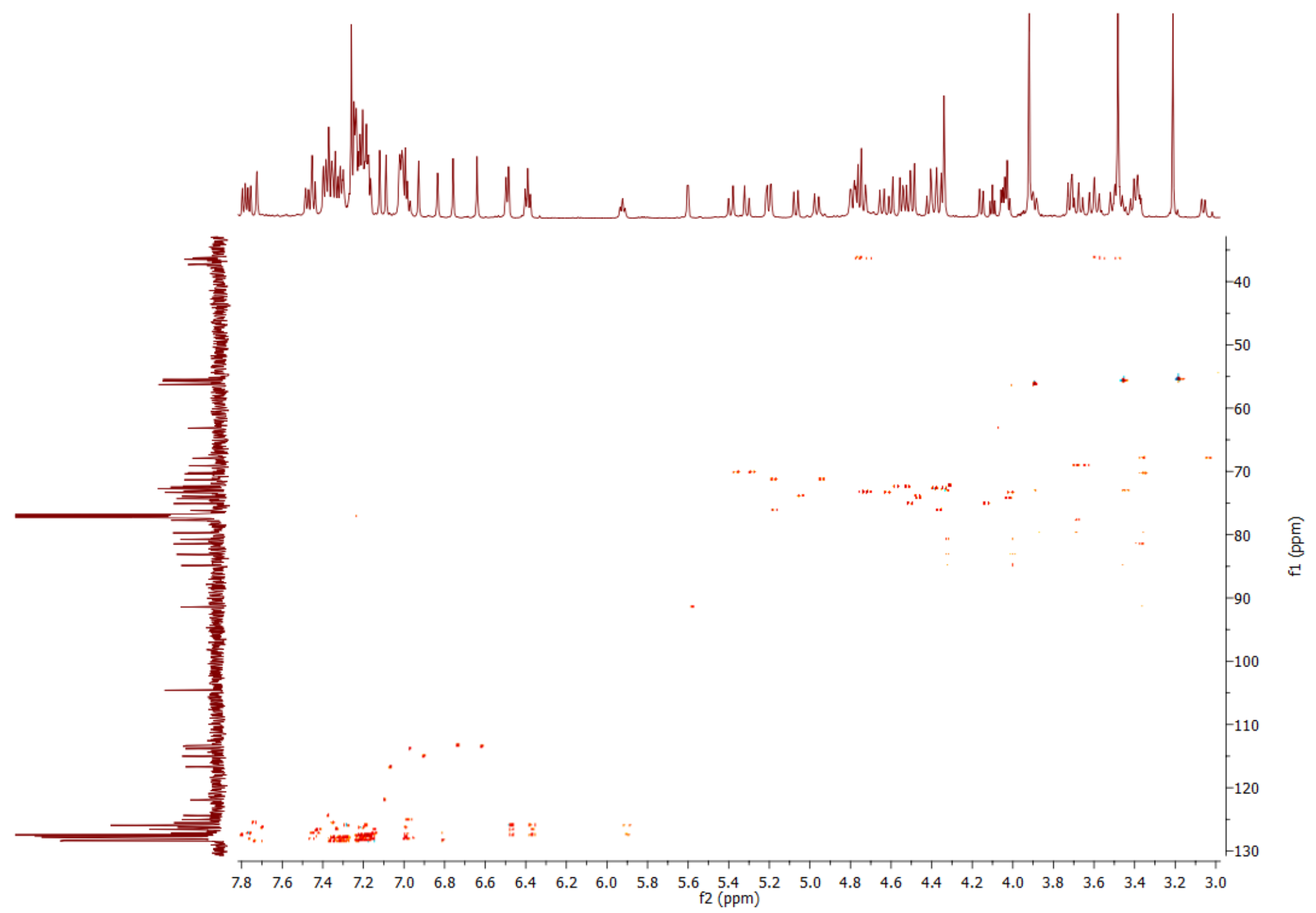

Figure S51. ${ }^{1} \mathrm{H}-{ }^{13} \mathrm{C}$ HSQC-TOCSY $\left(600 / 150 \mathrm{MHz}, \mathrm{CDCl}_{3}\right)$ spectrum of compound $\boldsymbol{M}-5 \mathbf{a}$. 


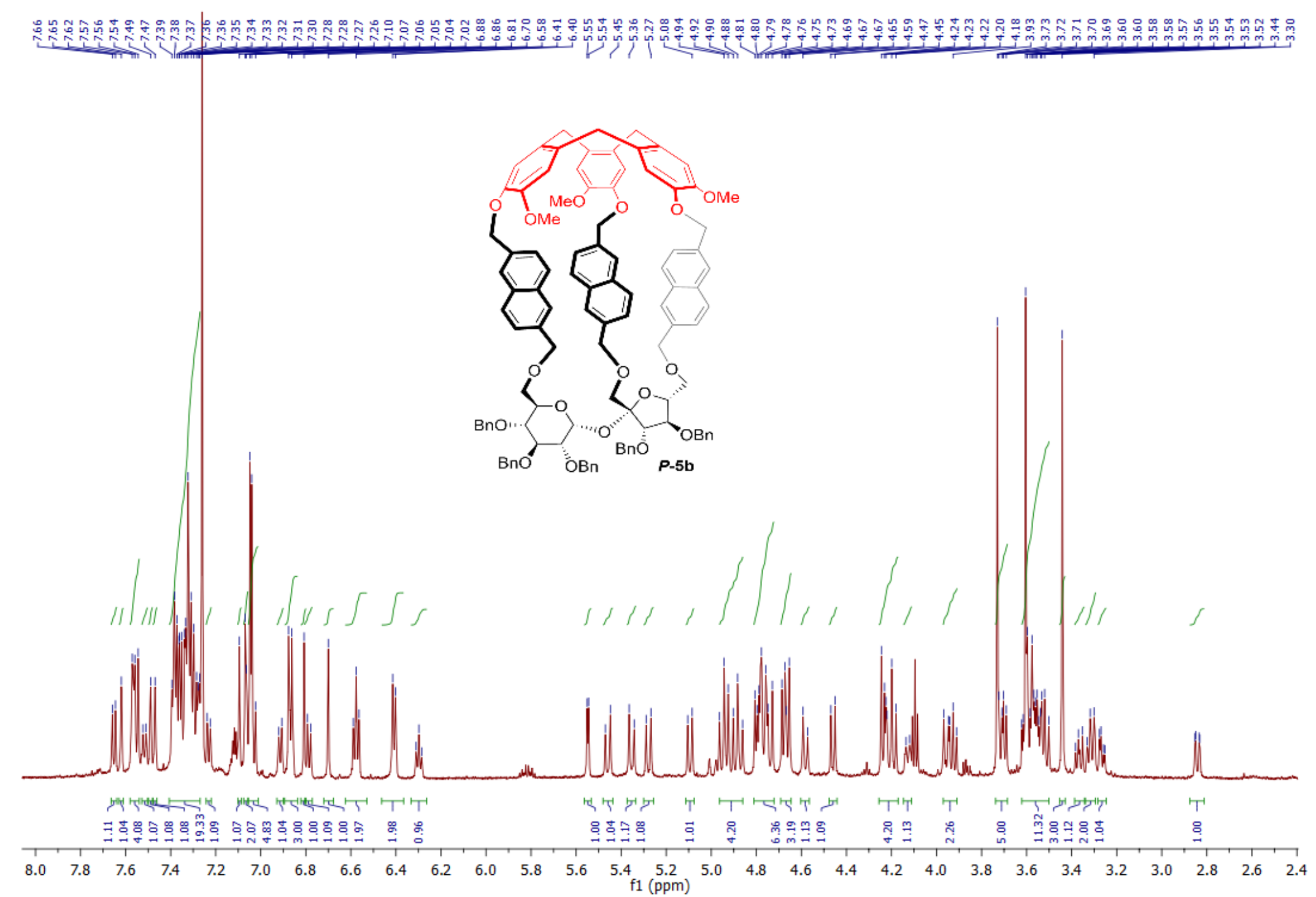

Figure S52. ${ }^{1} \mathrm{H}$ NMR $\left(600 \mathrm{MHz}, \mathrm{CDCl}_{3}\right)$ spectrum of compound $\boldsymbol{P}$-5b. 


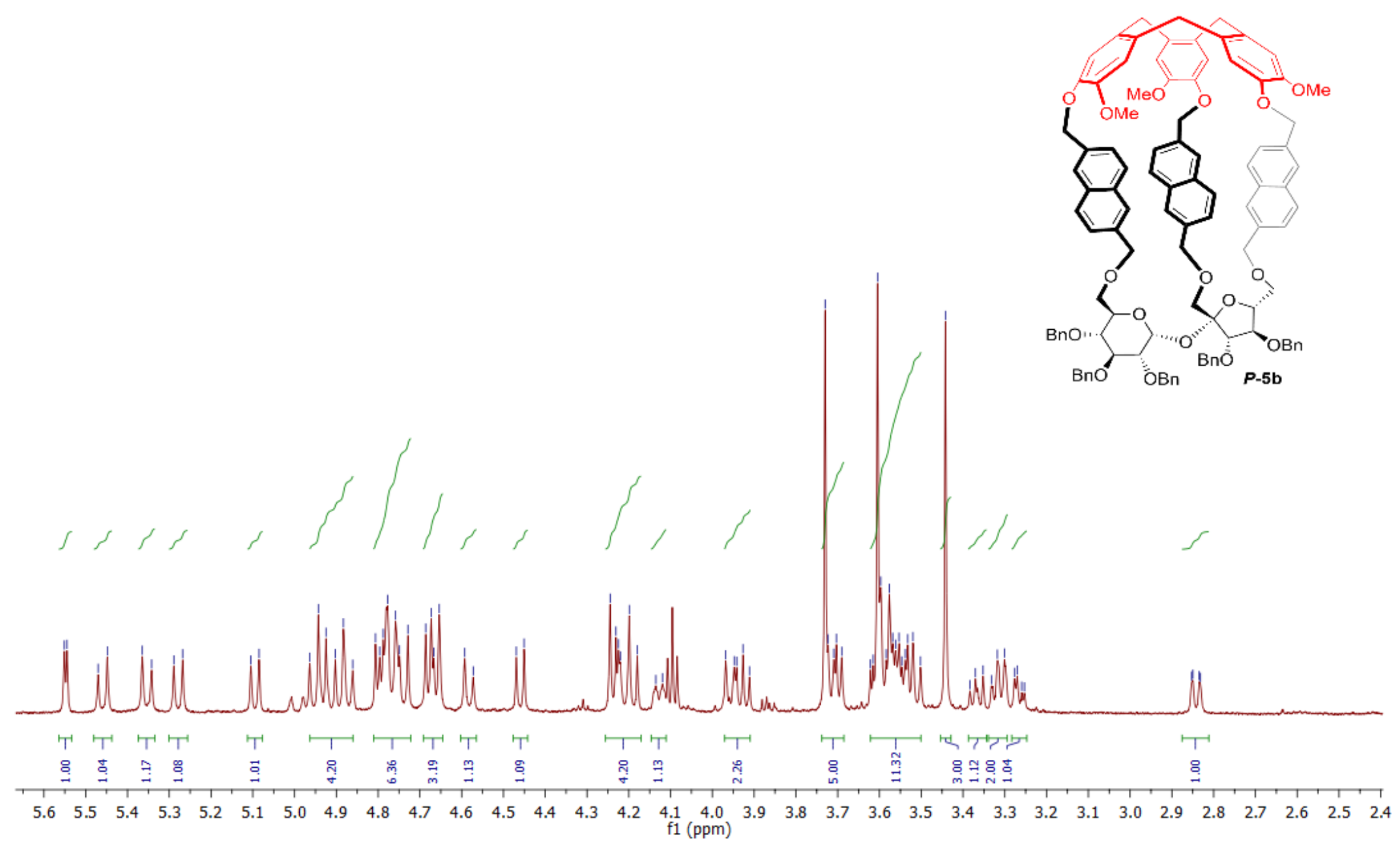

Figure S53. ${ }^{1} \mathrm{H}$ NMR $\left(600 \mathrm{MHz}, \mathrm{CDCl}_{3}\right.$ ) spectrum of compound $\boldsymbol{P}$-5b (aliphatic part). 


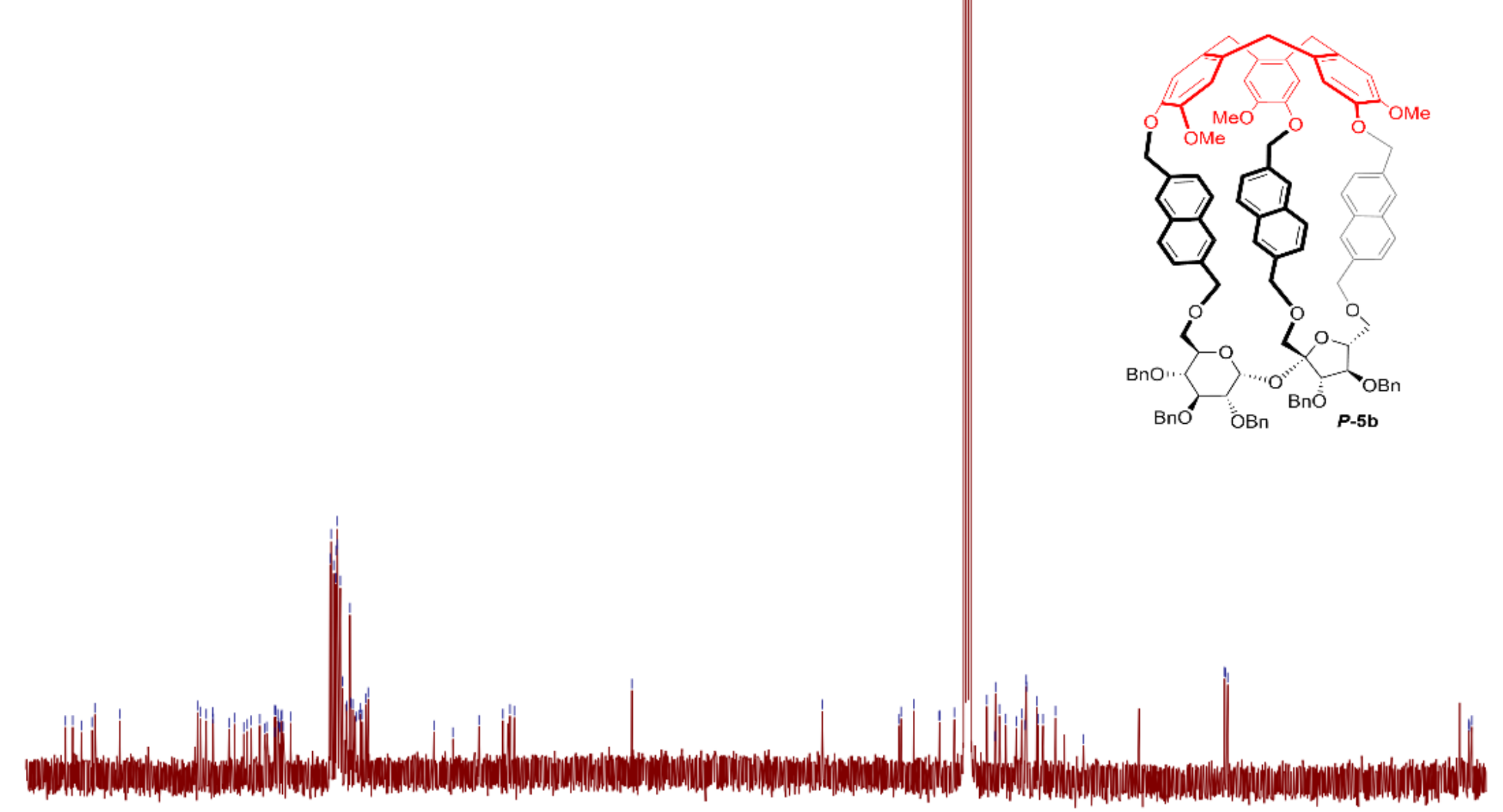

Figure S54. ${ }^{13} \mathrm{C}\left\{{ }^{1} \mathrm{H}\right\} \mathrm{NMR}\left(150 \mathrm{MHz}, \mathrm{CDCl}_{3}\right)$ spectrum of compound $\boldsymbol{P}-\mathbf{5 b}$. 

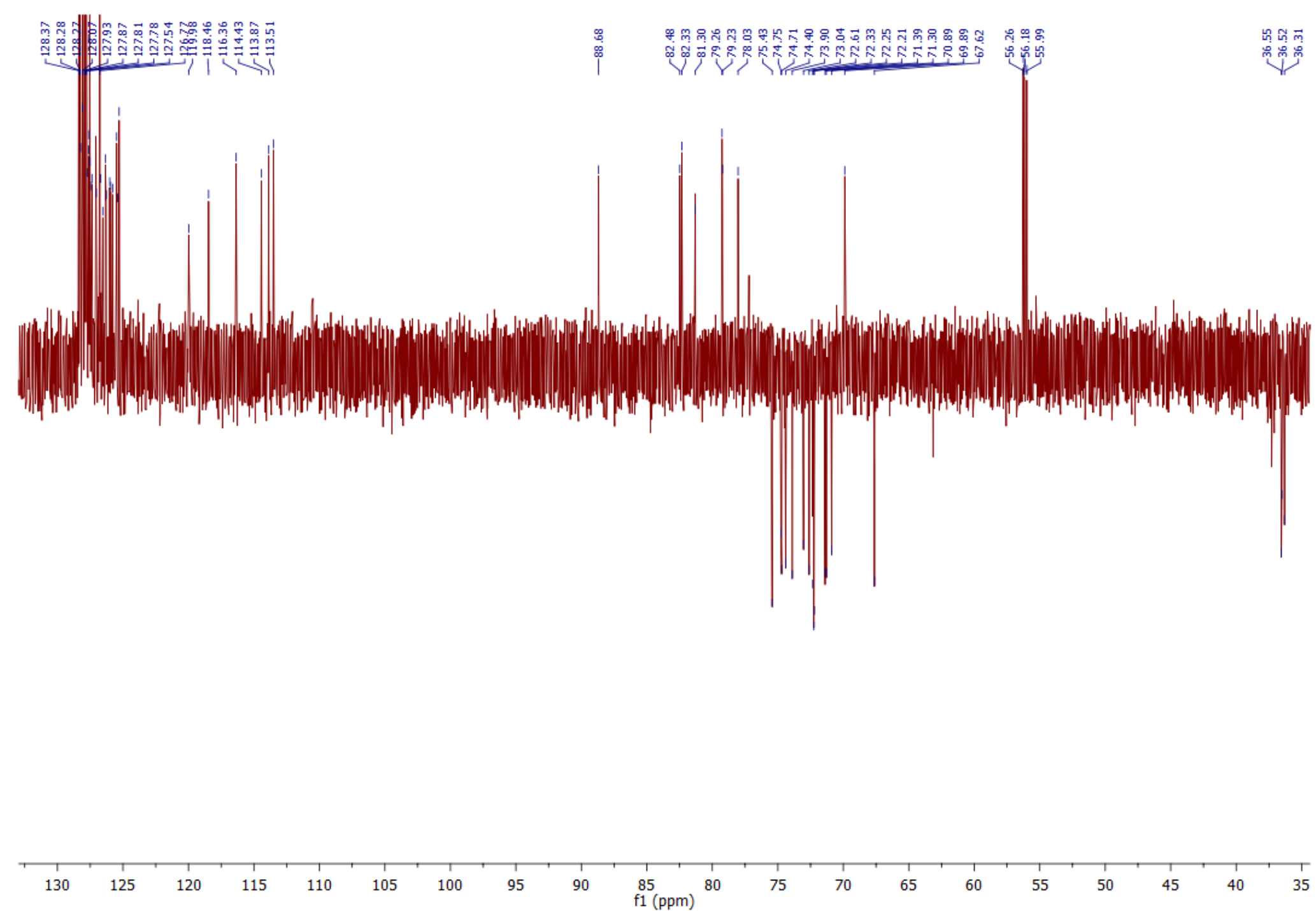

Figure S55. ${ }^{13} \mathrm{C}\left\{{ }^{1} \mathrm{H}\right\}$ DEPT (150 MHz, $\mathrm{CDCl}_{3}$ ) spectrum of compound $\boldsymbol{P}-\mathbf{5 b}$. 


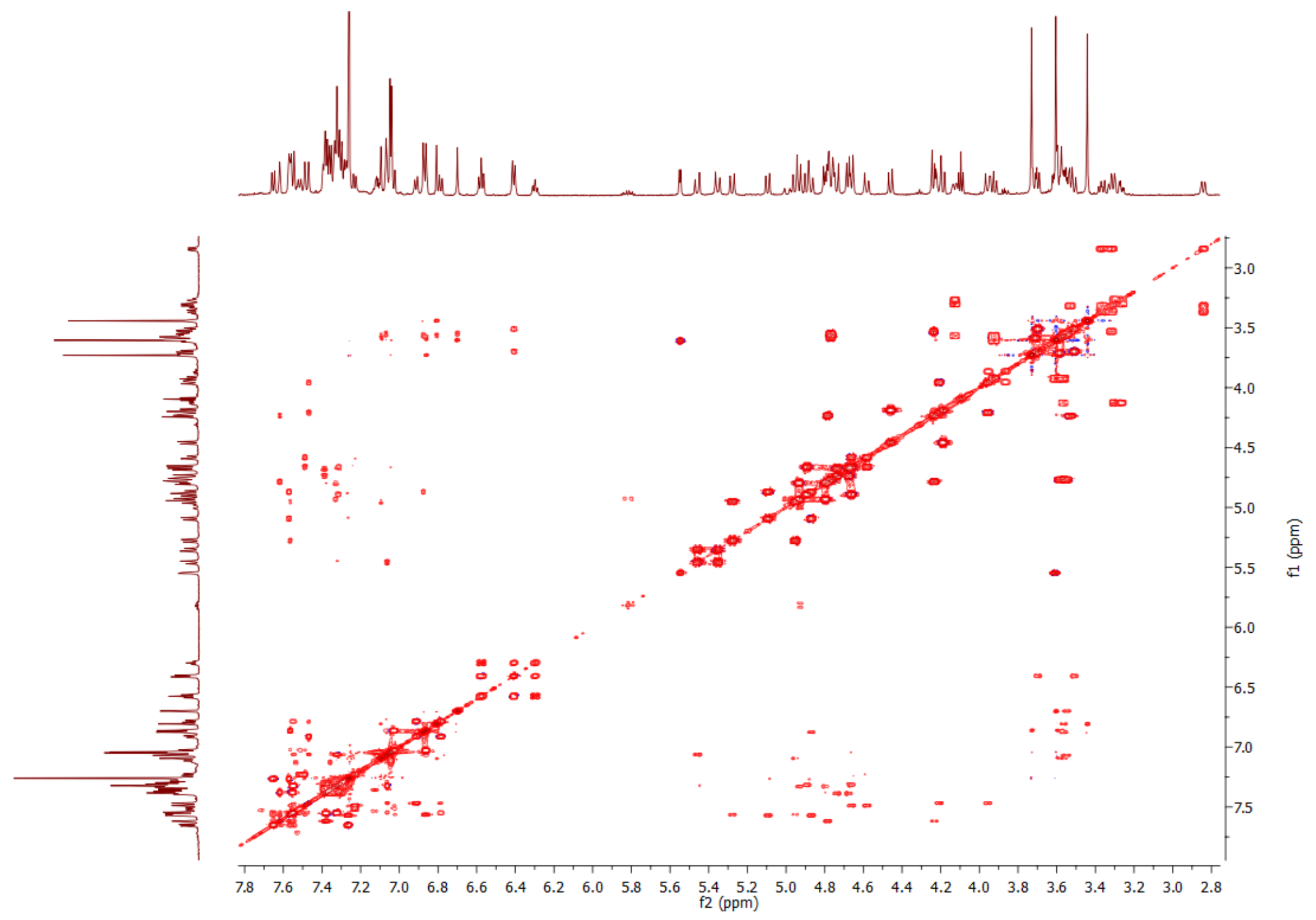

Figure S56. ${ }^{1} \mathrm{H}-{ }^{1} \mathrm{H} \operatorname{COSY}\left(600 \mathrm{MHz}, \mathrm{CDCl}_{3}\right)$ spectrum of compound $\boldsymbol{P}-\mathbf{5 b}$. 


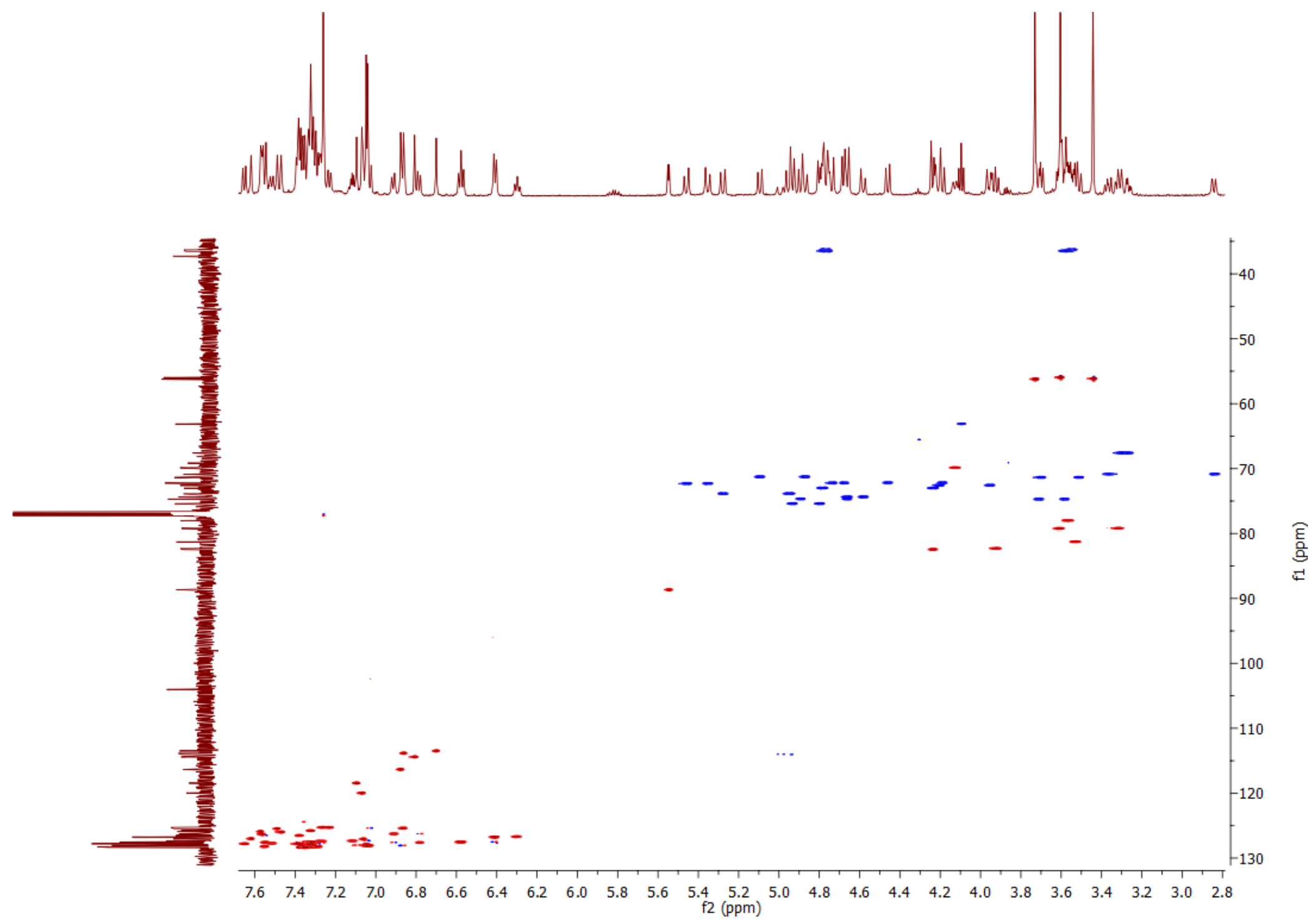

Figure S57. ${ }^{1} \mathrm{H}-{ }^{13} \mathrm{C}$ HSQC $\left(600 / 150 \mathrm{MHz}, \mathrm{CDCl}_{3}\right)$ spectrum of compound $\boldsymbol{P}-\mathbf{5 b}$. 


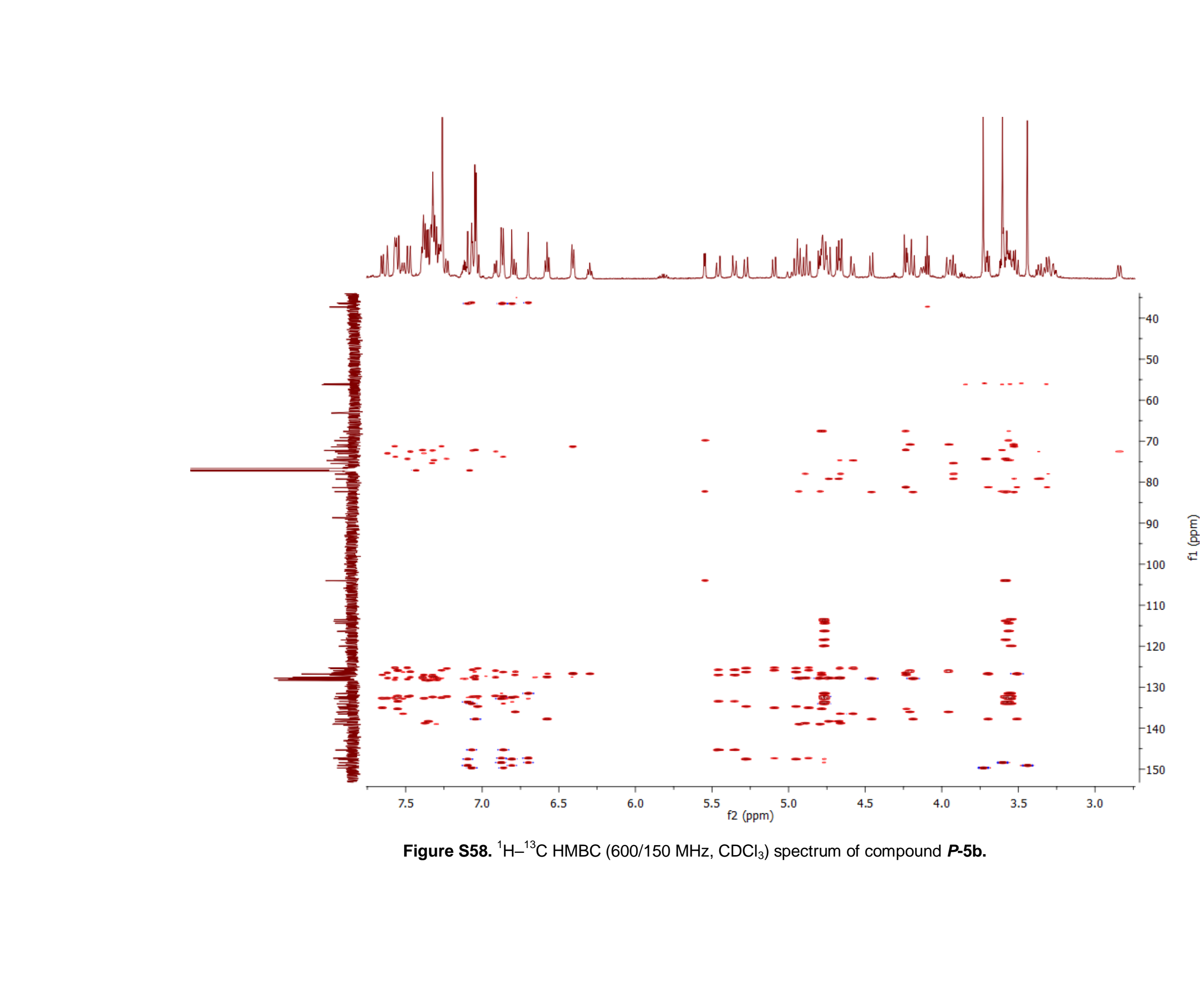




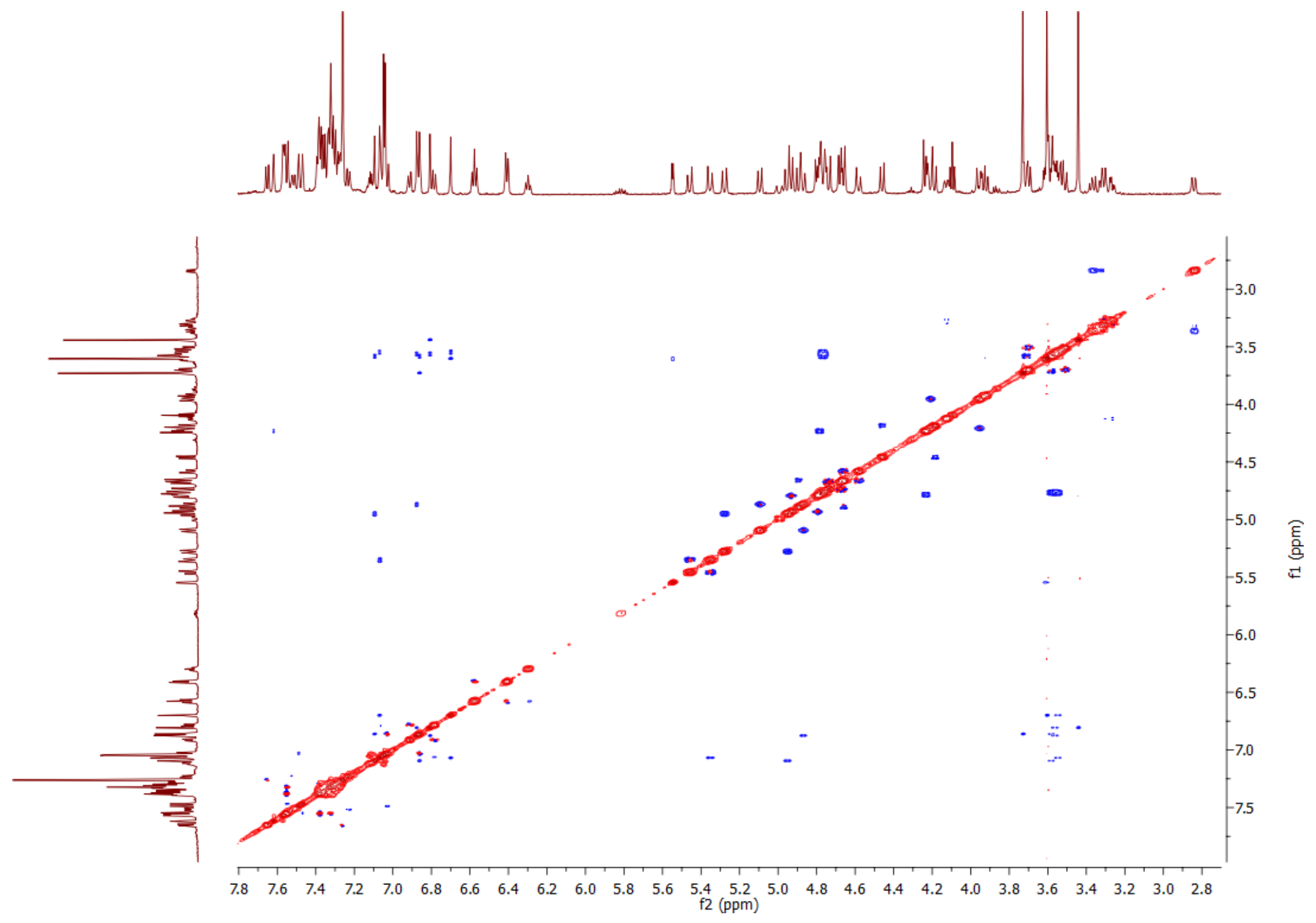

Figure S59. ${ }^{1} \mathrm{H}-{ }^{1} \mathrm{H}$ ROESY (600 MHz, $\mathrm{CDCl}_{3}$ ) spectrum of compound $\boldsymbol{P}-\mathbf{5 b}$. 


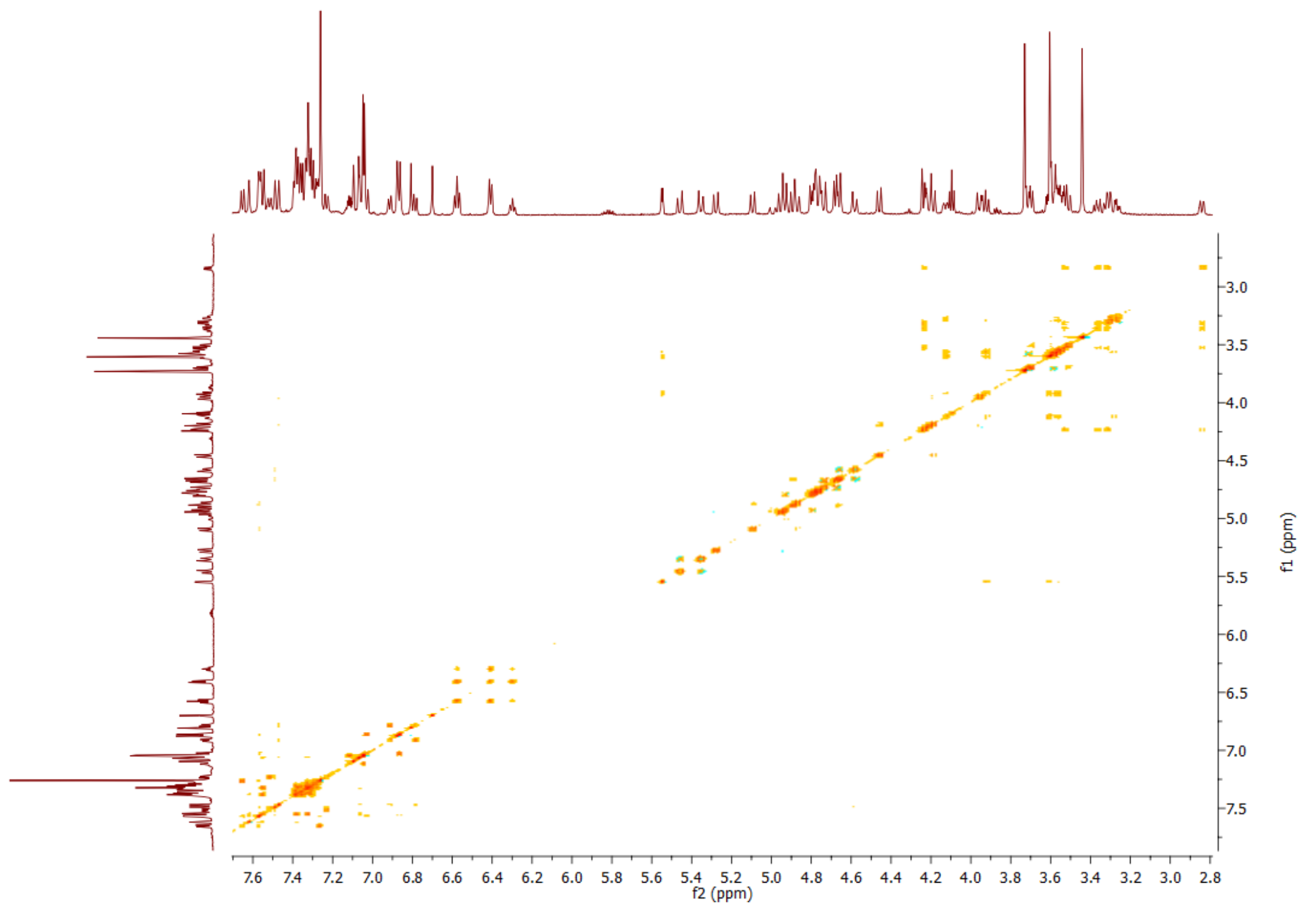

Figure S60. ${ }^{1} \mathrm{H}-{ }^{1} \mathrm{H}$ TOCSY (600 MHz, $\mathrm{CDCl}_{3}$ ) spectrum of compound $\mathbf{P}-\mathbf{5 b}$. 


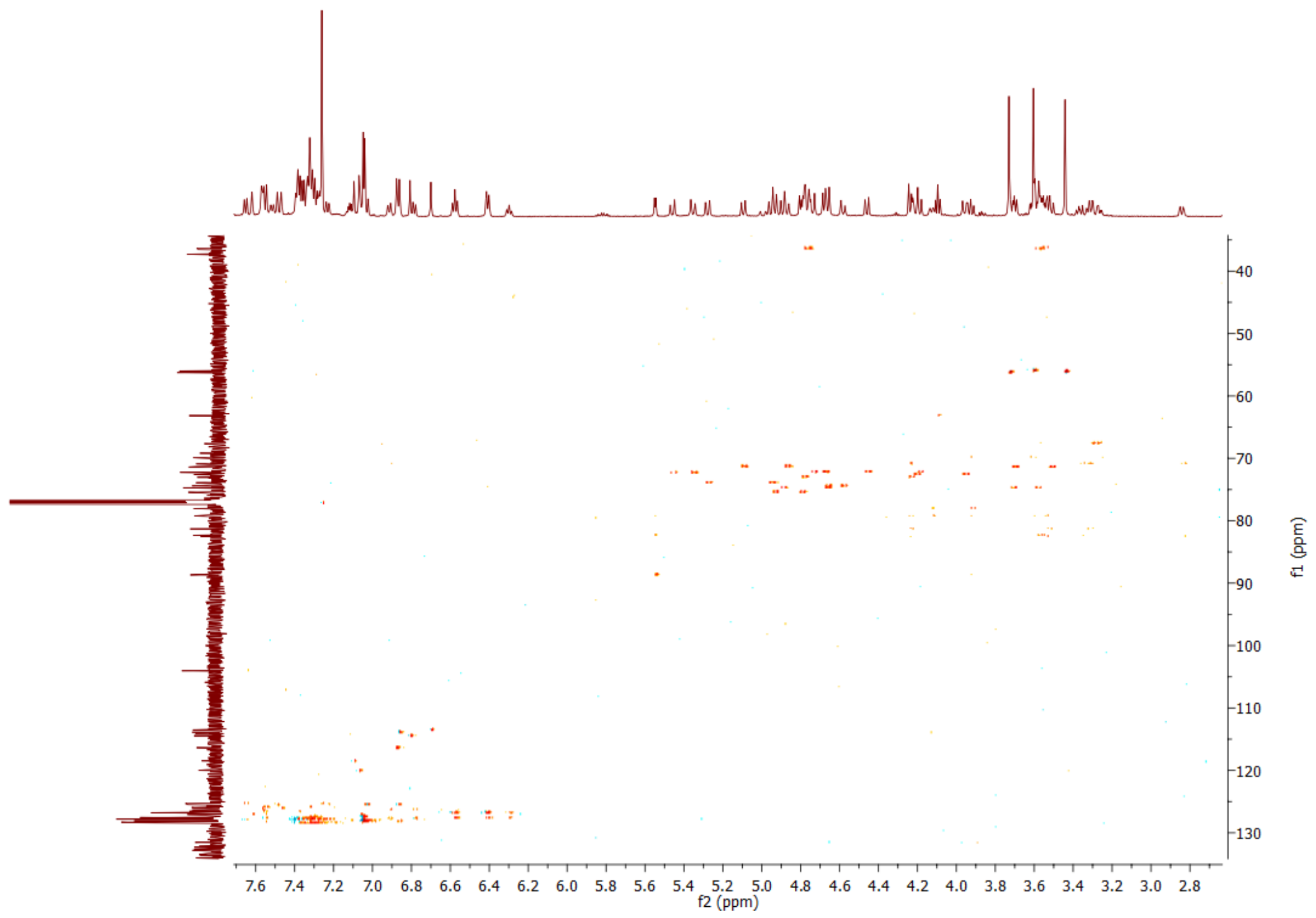

Figure S61. ${ }^{1} \mathrm{H}-{ }^{13} \mathrm{C}$ HSQC-TOCSY $\left(600 / 150 \mathrm{MHz}, \mathrm{CDCl}_{3}\right)$ spectrum of compound $\boldsymbol{P}-\mathbf{5 b}$.

S53 


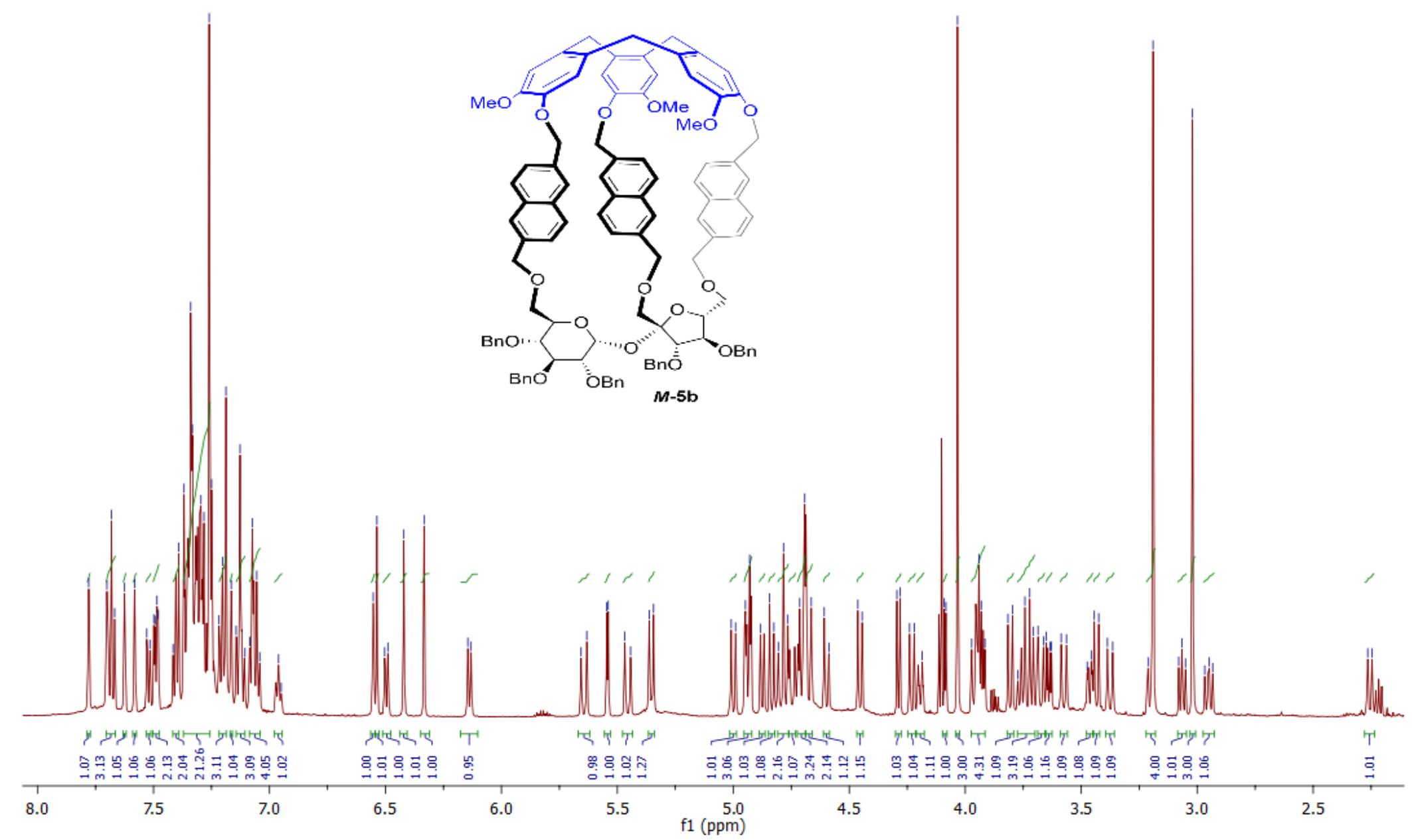

Figure S62. ${ }^{1} \mathrm{H}$ NMR $\left(600 \mathrm{MHz}, \mathrm{CDCl}_{3}\right)$ spectrum of compound $\boldsymbol{M}-5 \mathbf{b}$. 


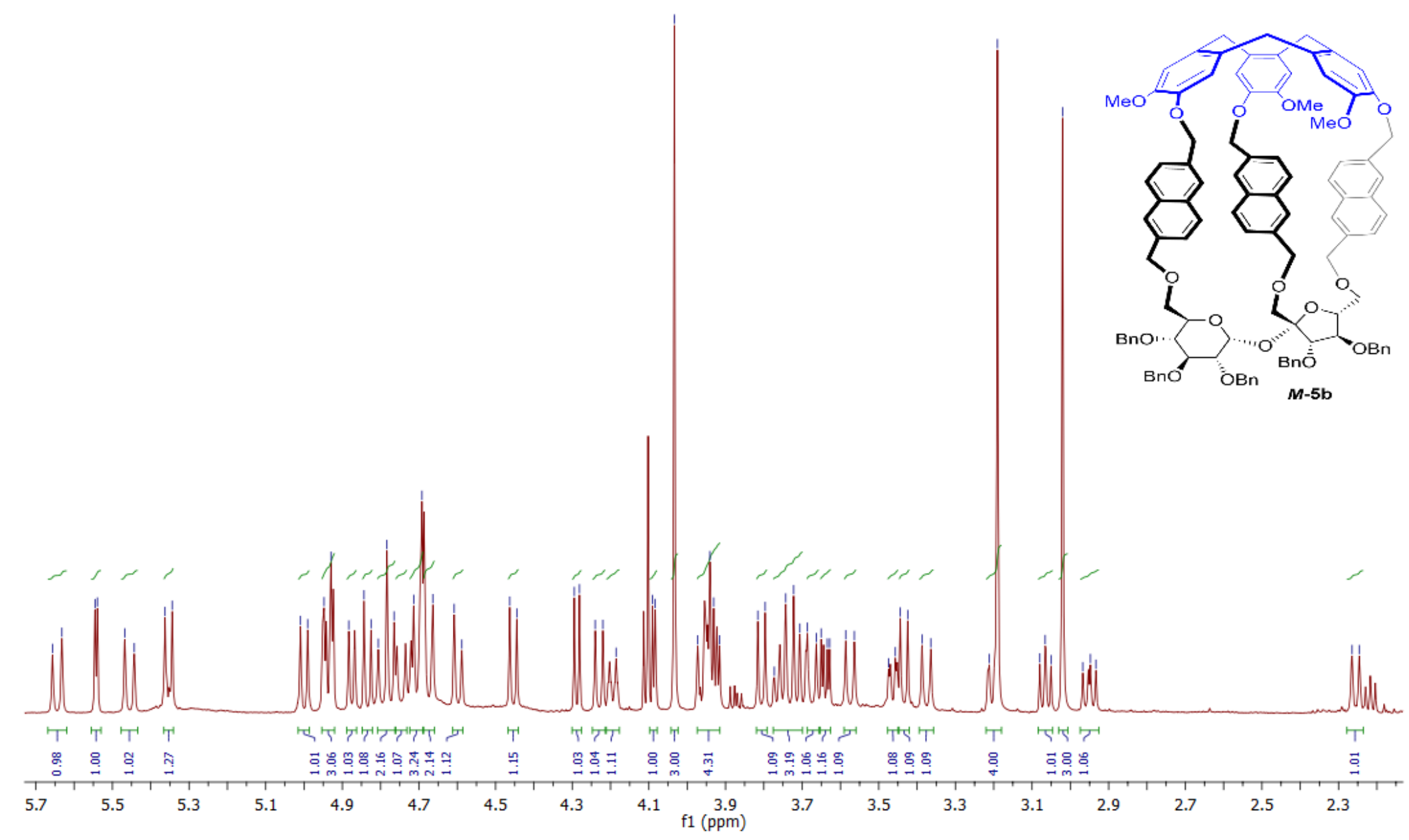

Figure S63. ${ }^{1} \mathrm{H}$ NMR $\left(600 \mathrm{MHz}, \mathrm{CDCl}_{3}\right)$ spectrum of compound $\boldsymbol{M}$-5b (aliphatic part). 

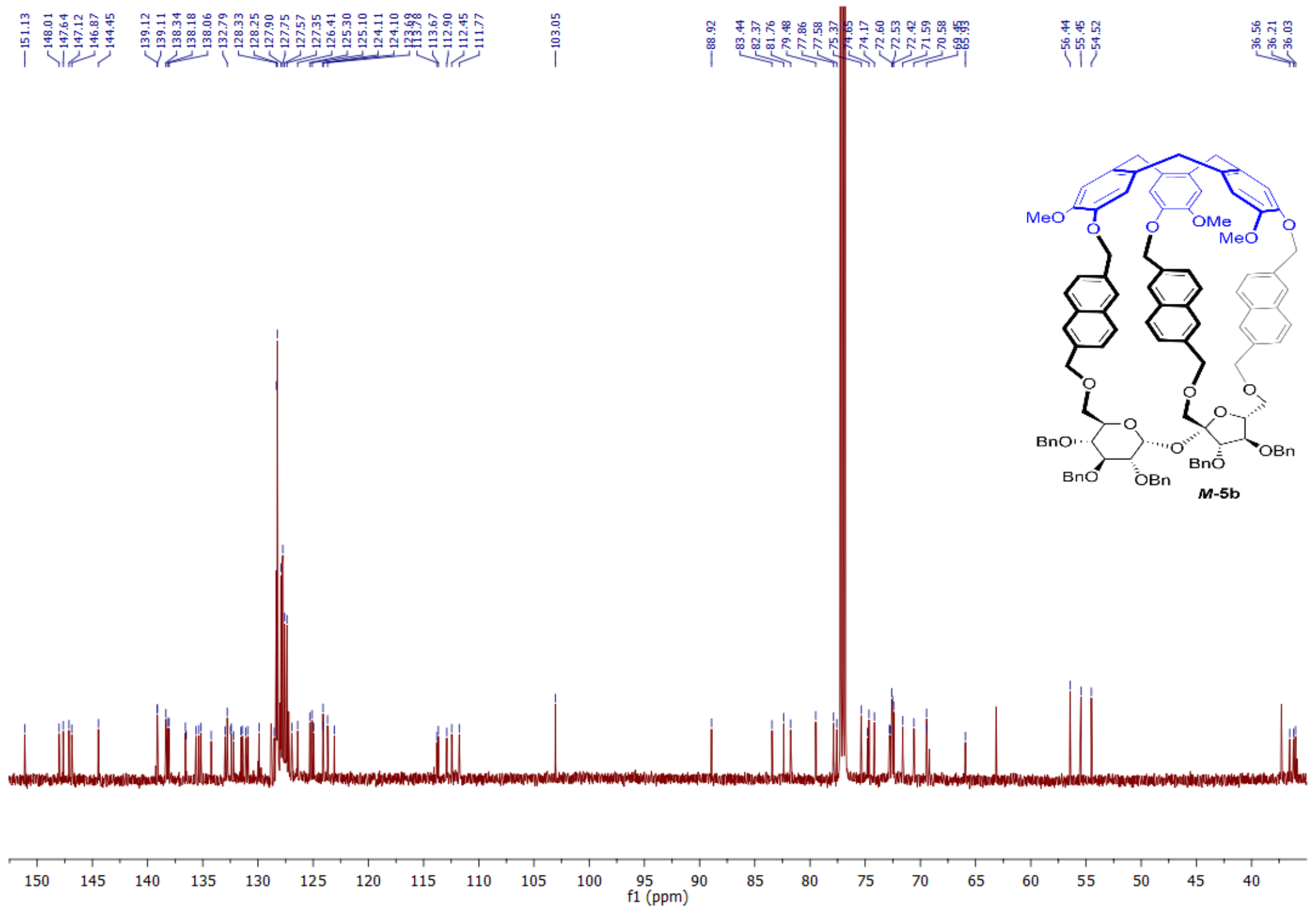

Figure S64. ${ }^{13} \mathrm{C}\left\{{ }^{1} \mathrm{H}\right\} \mathrm{NMR}\left(150 \mathrm{MHz}, \mathrm{CDCl}_{3}\right)$ spectrum of compound $\boldsymbol{M}-\mathbf{5 b}$. 


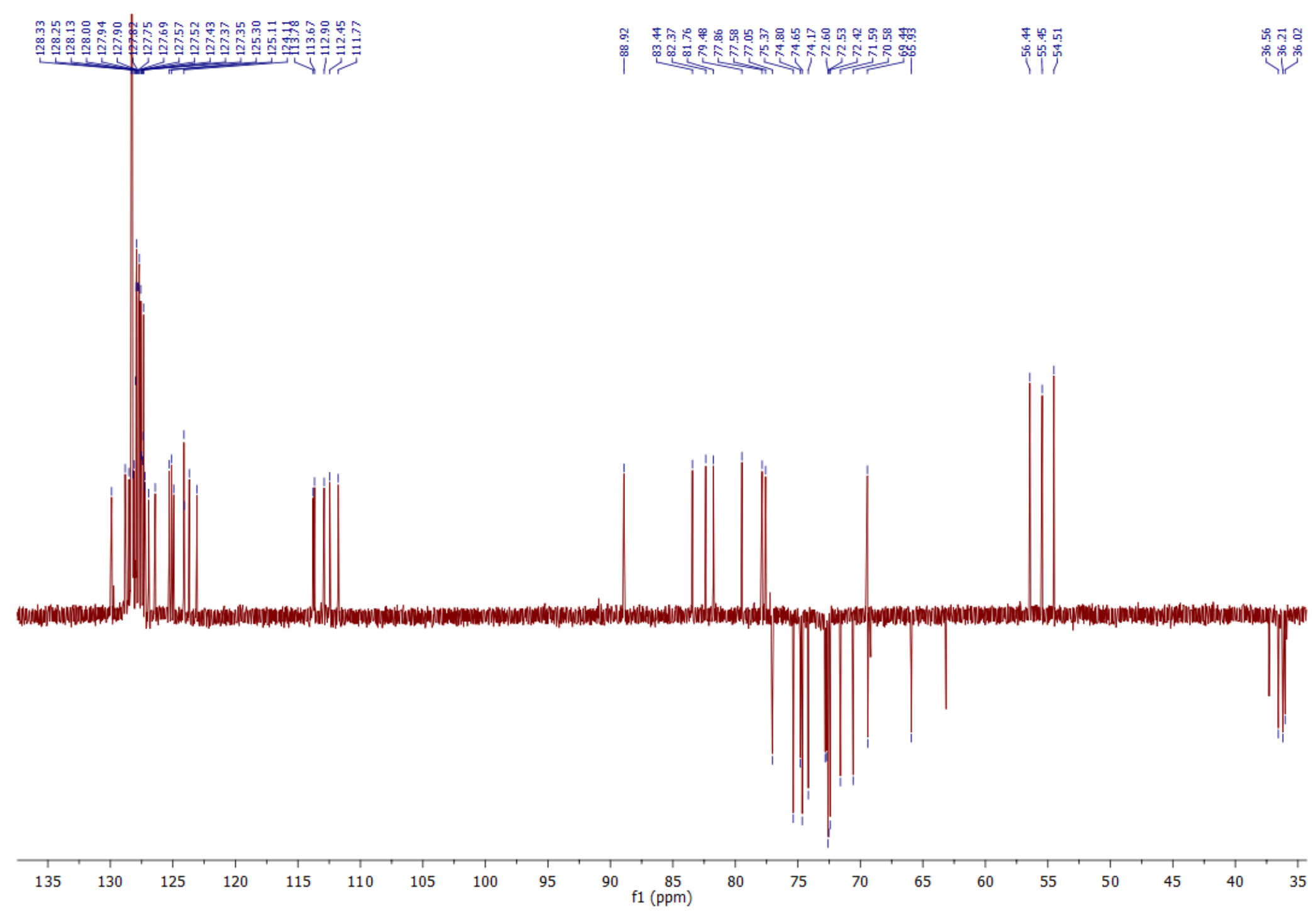

Figure S65. ${ }^{13} \mathrm{C}\left\{{ }^{1} \mathrm{H}\right\}$ DEPT (150 MHz, $\mathrm{CDCl}_{3}$ ) spectrum of compound $\boldsymbol{M}-\mathbf{5 b}$. 


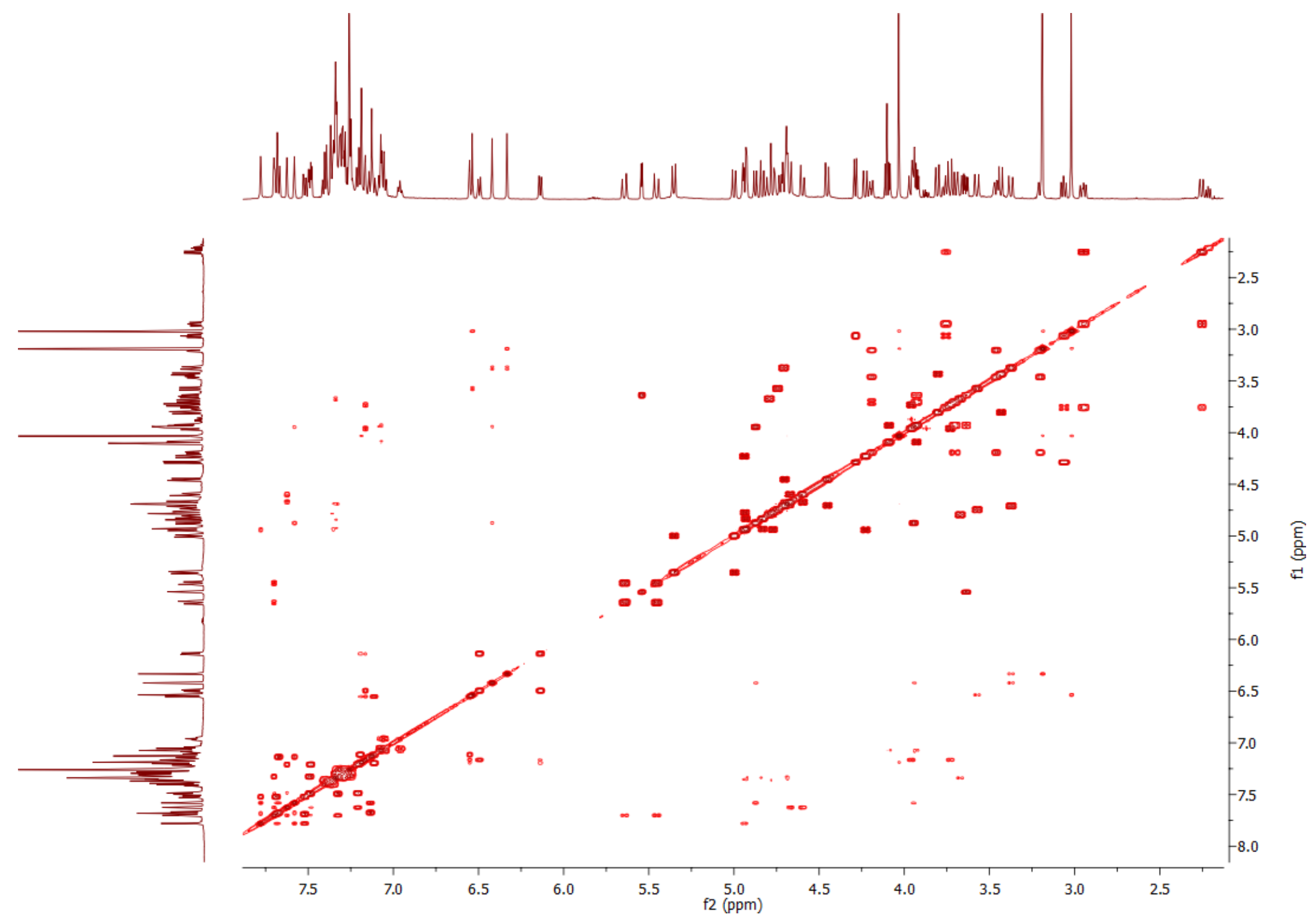

Figure S66. ${ }^{1} \mathrm{H}-{ }^{1} \mathrm{H}$ COSY $\left(600 \mathrm{MHz}, \mathrm{CDCl}_{3}\right)$ spectrum of compound $\boldsymbol{M}-\mathbf{5} \mathbf{b}$. 


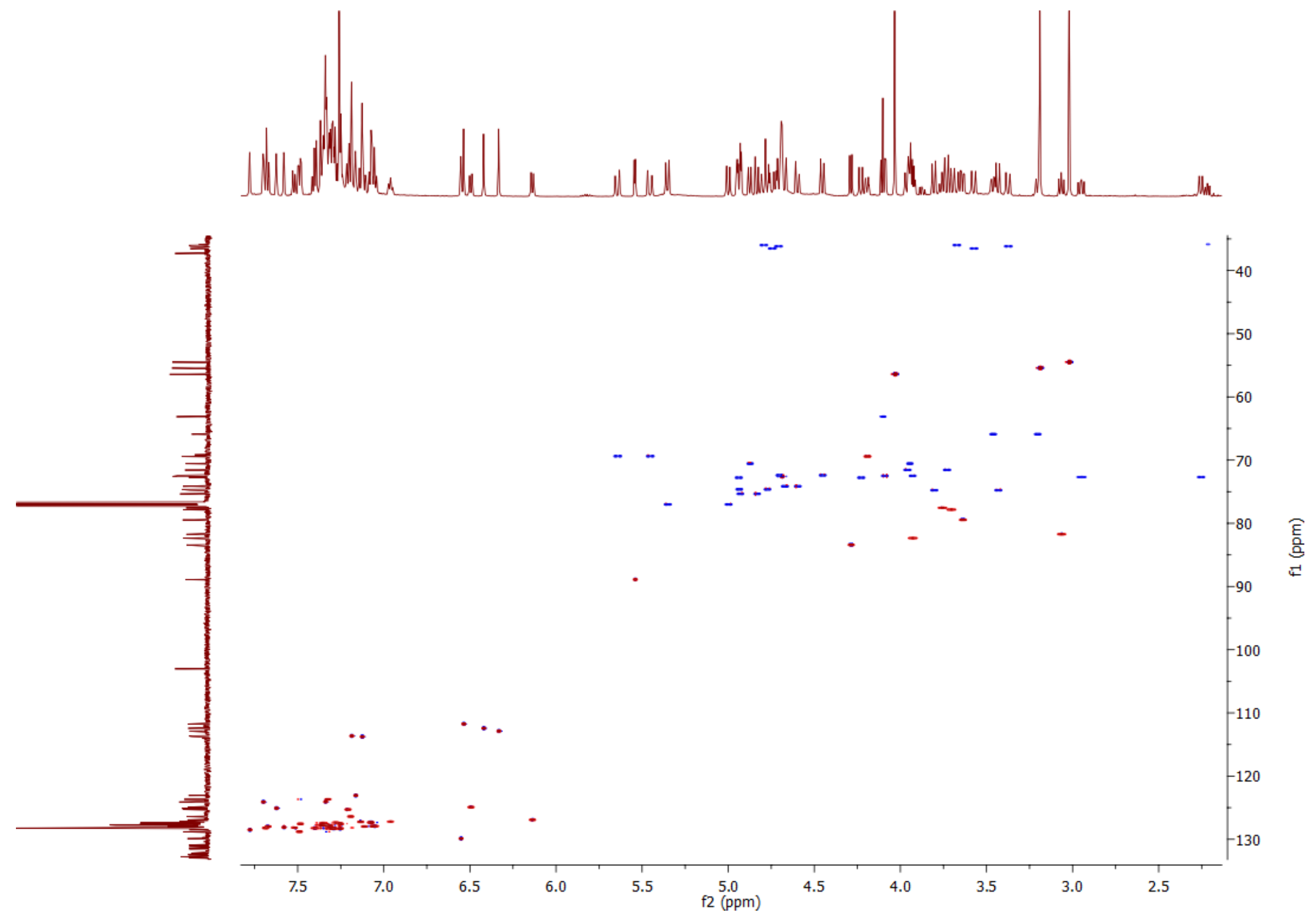

Figure S67. ${ }^{1} \mathrm{H}-{ }^{13} \mathrm{C}$ HSQC $\left(600 / 150 \mathrm{MHz}, \mathrm{CDCl}_{3}\right)$ spectrum of compound $\boldsymbol{M}-5 \mathbf{b}$. 


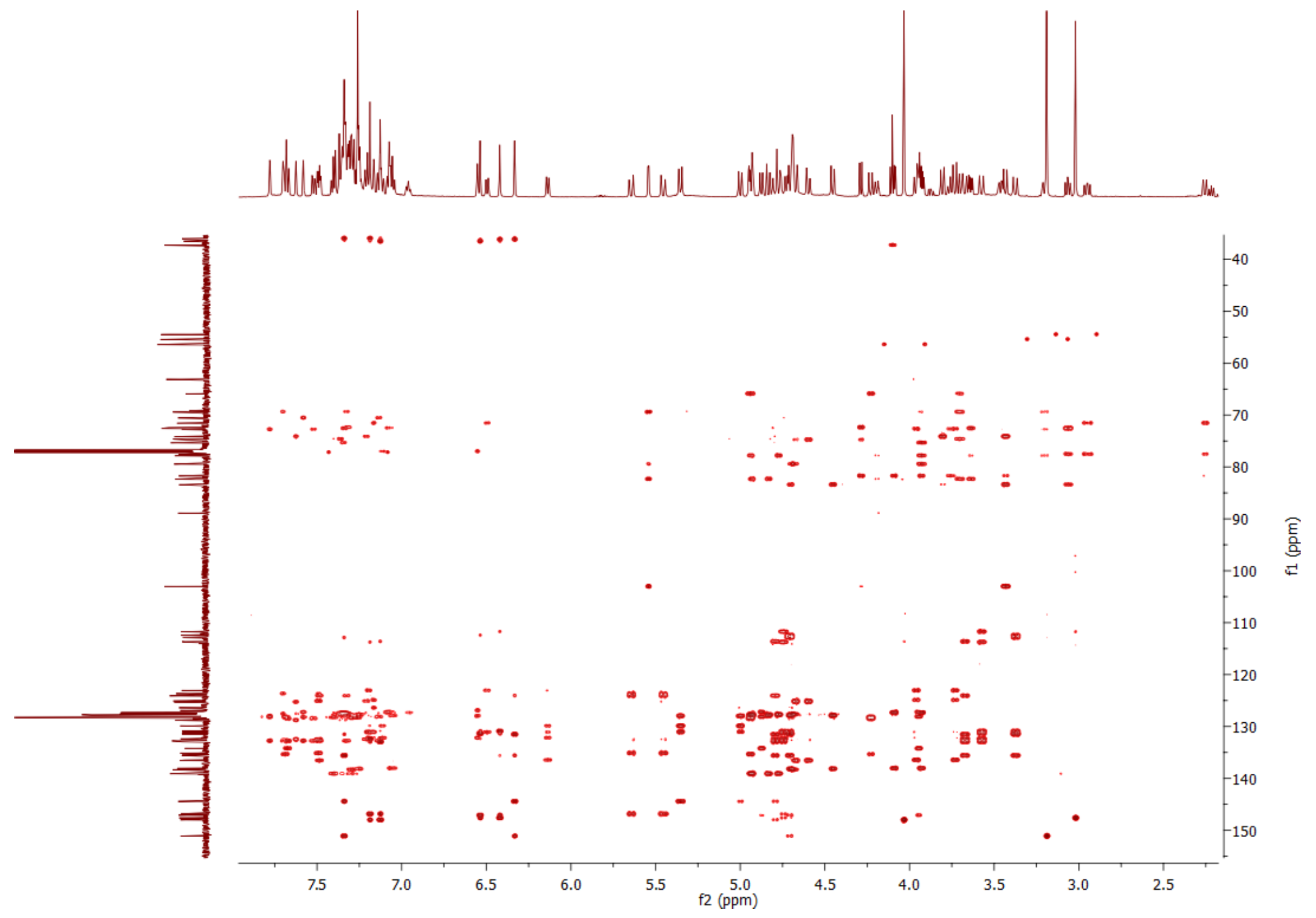

Figure S68. ${ }^{1} \mathrm{H}^{13}{ }^{13} \mathrm{HMBC}\left(600 / 150 \mathrm{MHz}, \mathrm{CDCl}_{3}\right)$ spectrum of compound $\boldsymbol{M}-5 \mathbf{b}$. 


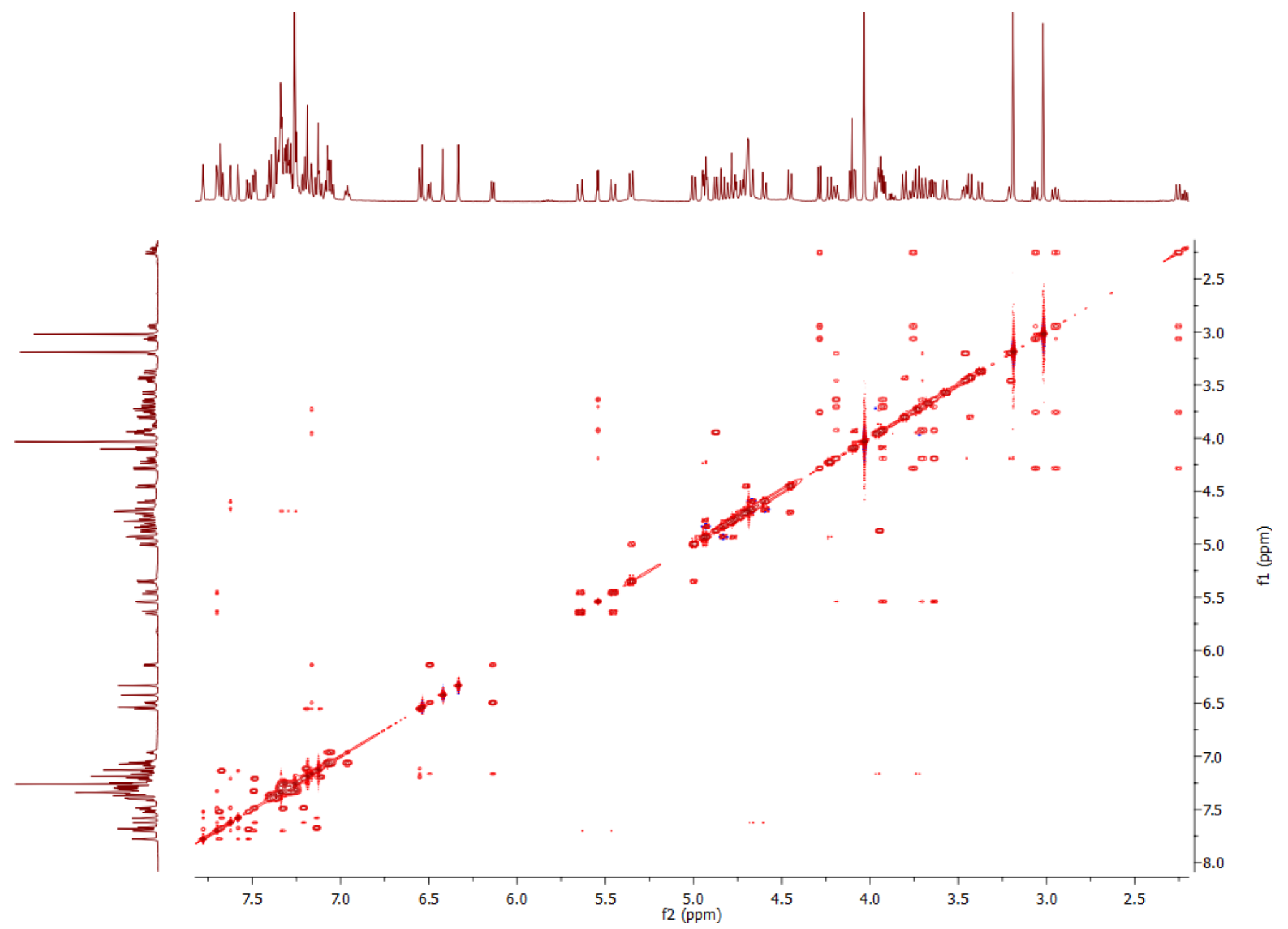

Figure S69. ${ }^{1} \mathrm{H}-{ }^{1} \mathrm{H}$ TOCSY $\left(600 \mathrm{MHz}, \mathrm{CDCl}_{3}\right)$ spectrum of compound $\boldsymbol{M}-\mathbf{5 b}$. 


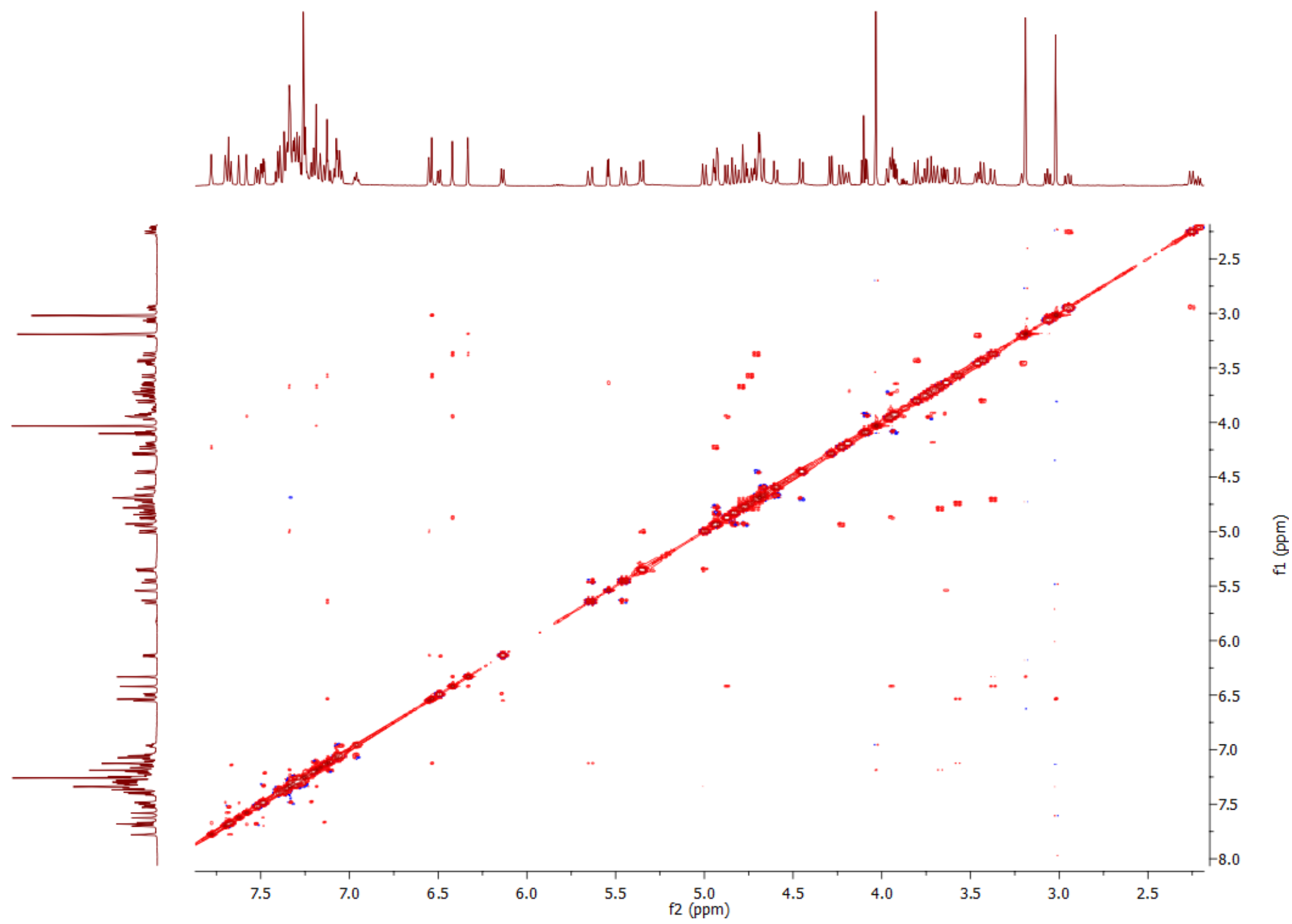

Figure $\mathbf{S} 70 .{ }^{1} \mathrm{H}-{ }^{1} \mathrm{H}$ NOESY $\left(600 \mathrm{MHz}, \mathrm{CDCl}_{3}\right.$ ) spectrum of compound $\boldsymbol{M}-\mathbf{5 b}$. 


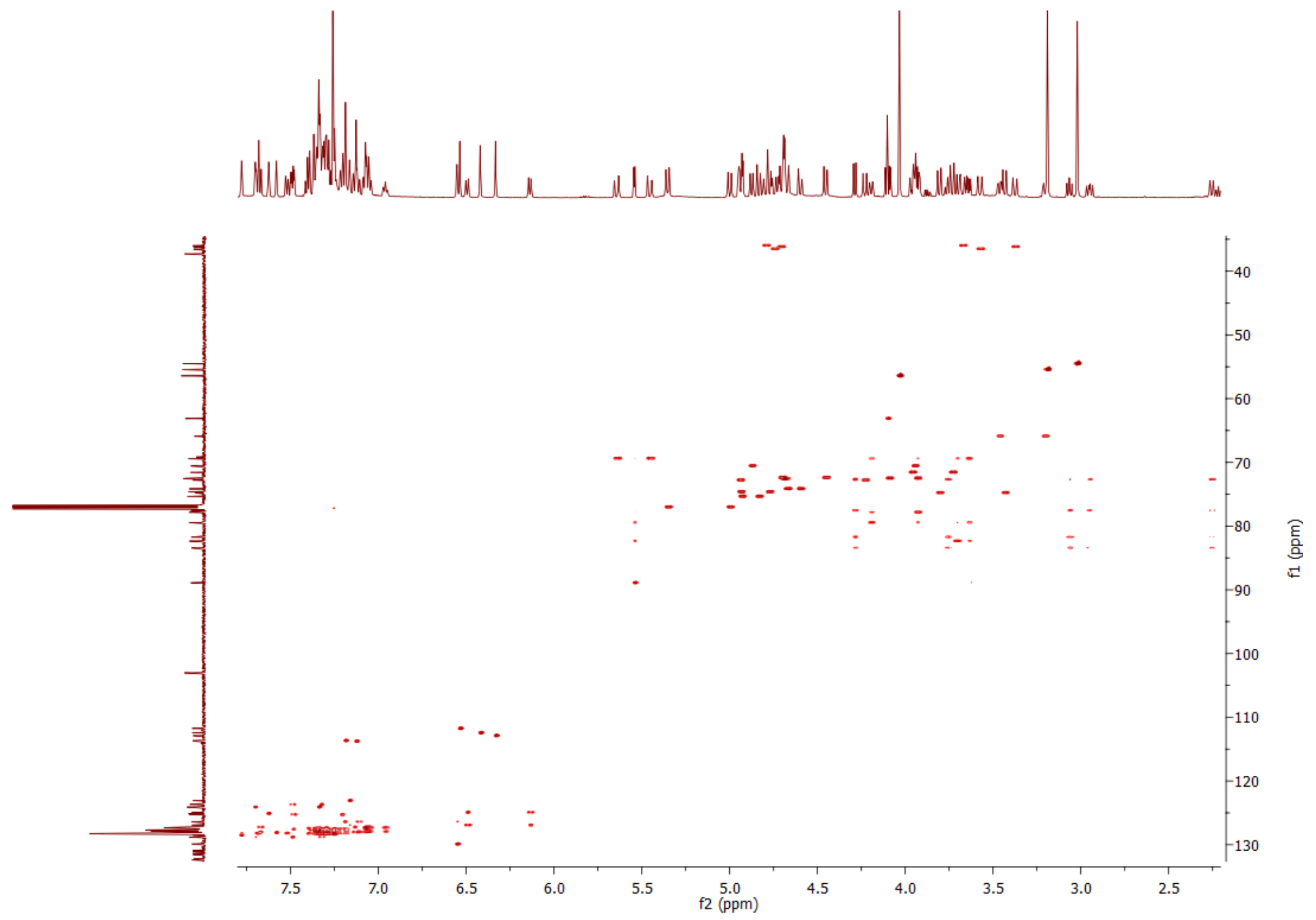

Figure S71. ${ }^{1} \mathrm{H}-{ }^{13} \mathrm{C}$ HSQC-TOCSY $\left(600 / 150 \mathrm{MHz}, \mathrm{CDCl}_{3}\right)$ spectrum of compound $\boldsymbol{M}-\mathbf{5 b}$. 


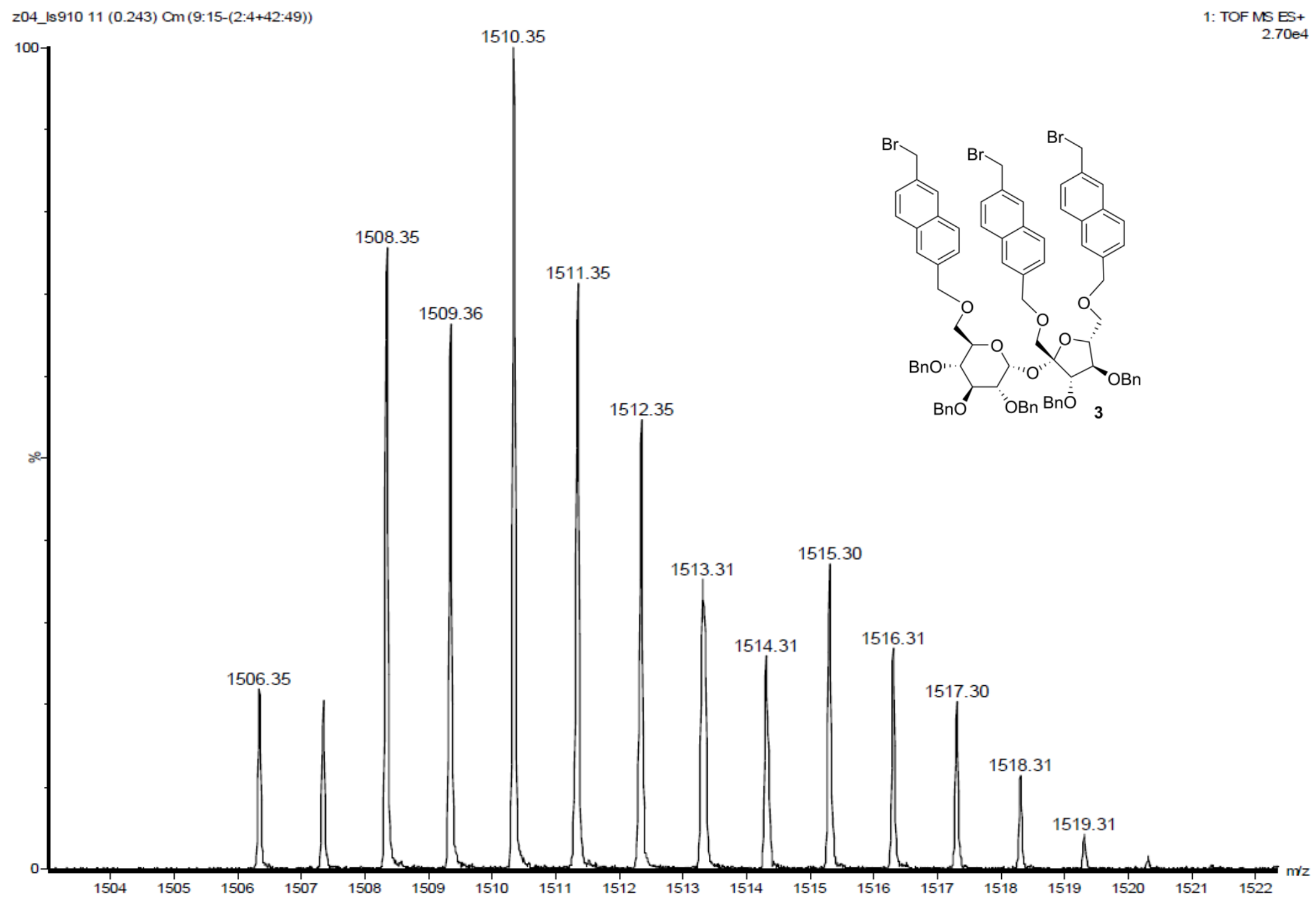

Figure S72. ESI-MS spectrum of compound 3.

S64 
Elements Used:

\begin{tabular}{l|l|l|l|l|l|c|c|c|c|c}
\hline Mass & Calc. Mass & $\mathrm{mDa}$ & PPM & DBE & Formula & $\mathrm{C}$ & $\mathrm{H}$ & $\mathrm{O}$ & $\mathrm{Na}$ & \\
\hline 1679.6844 & 1679.6858 & -1.4 & -0.8 & 57.5 & C107 H100 017 Na & 107 & 100 & 17 & 1 & \\
& 1679.6799 & 4.5 & 2.7 & 66.5 & C114 H96 012 Na & 114 & 96 & 12 & 1 & \\
& 1679.6893 & -4.9 & -2.9 & 79.5 & C125 H92 O4 Na & 125 & 92 & 4 & 1
\end{tabular}

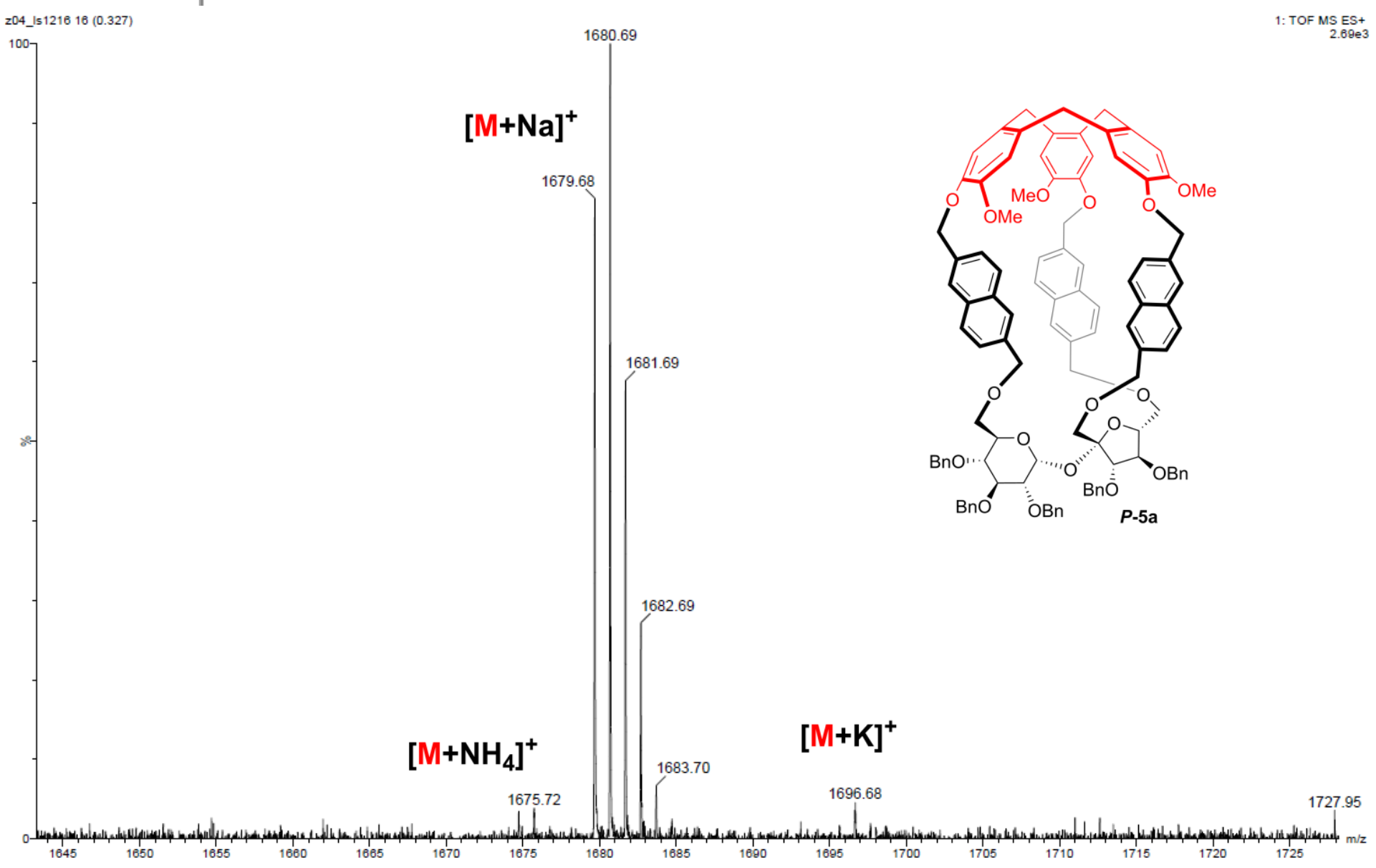

Figure S73. HRMS spectrum of compound $\boldsymbol{P}$-5a. 


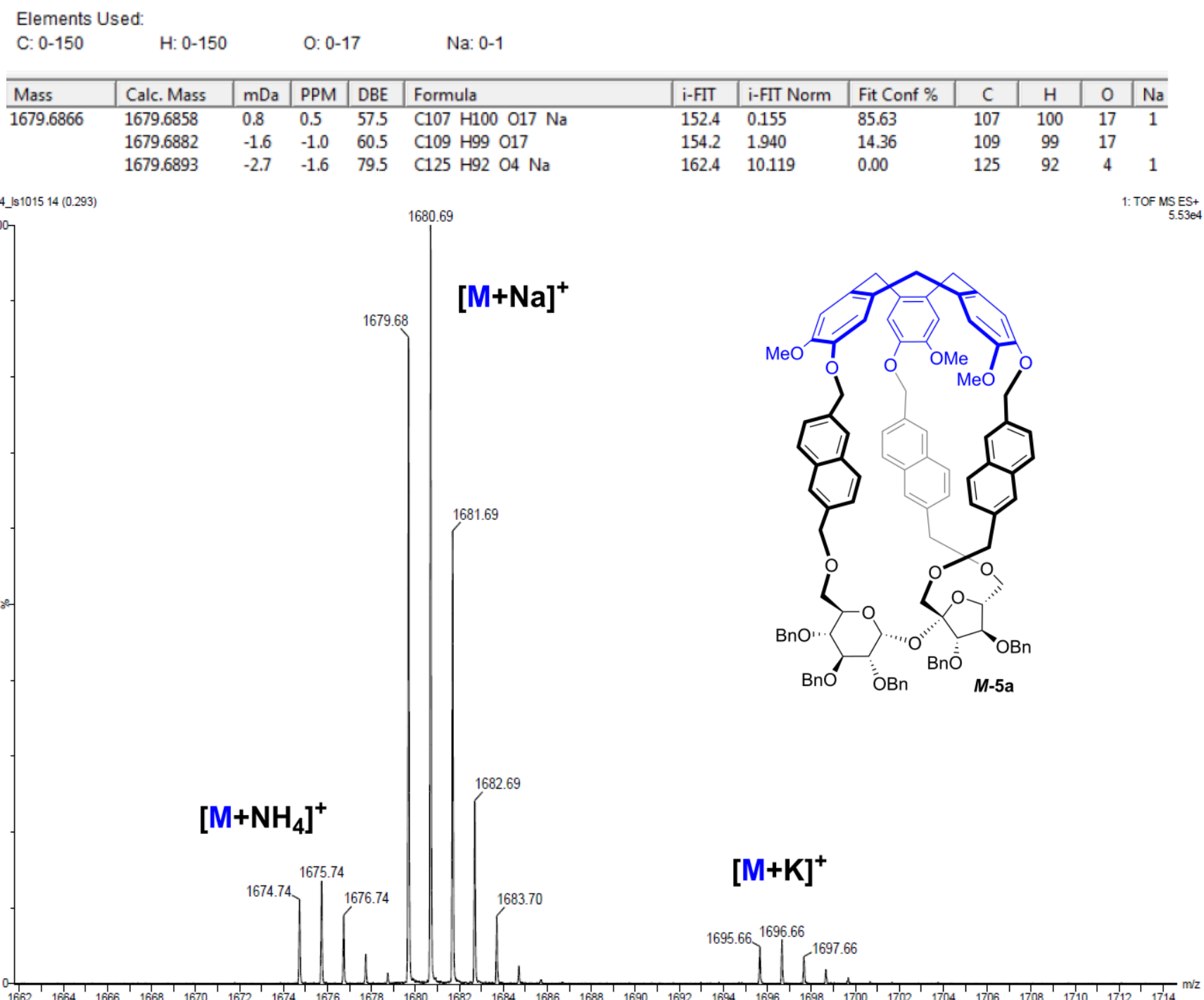

Figure S74. HRMS spectrum of compound $\boldsymbol{M}-\mathbf{5}$ a. 
Elements Used:
C: $0-150$
H: $0-150$
0: $0-20$
Na: 1-1

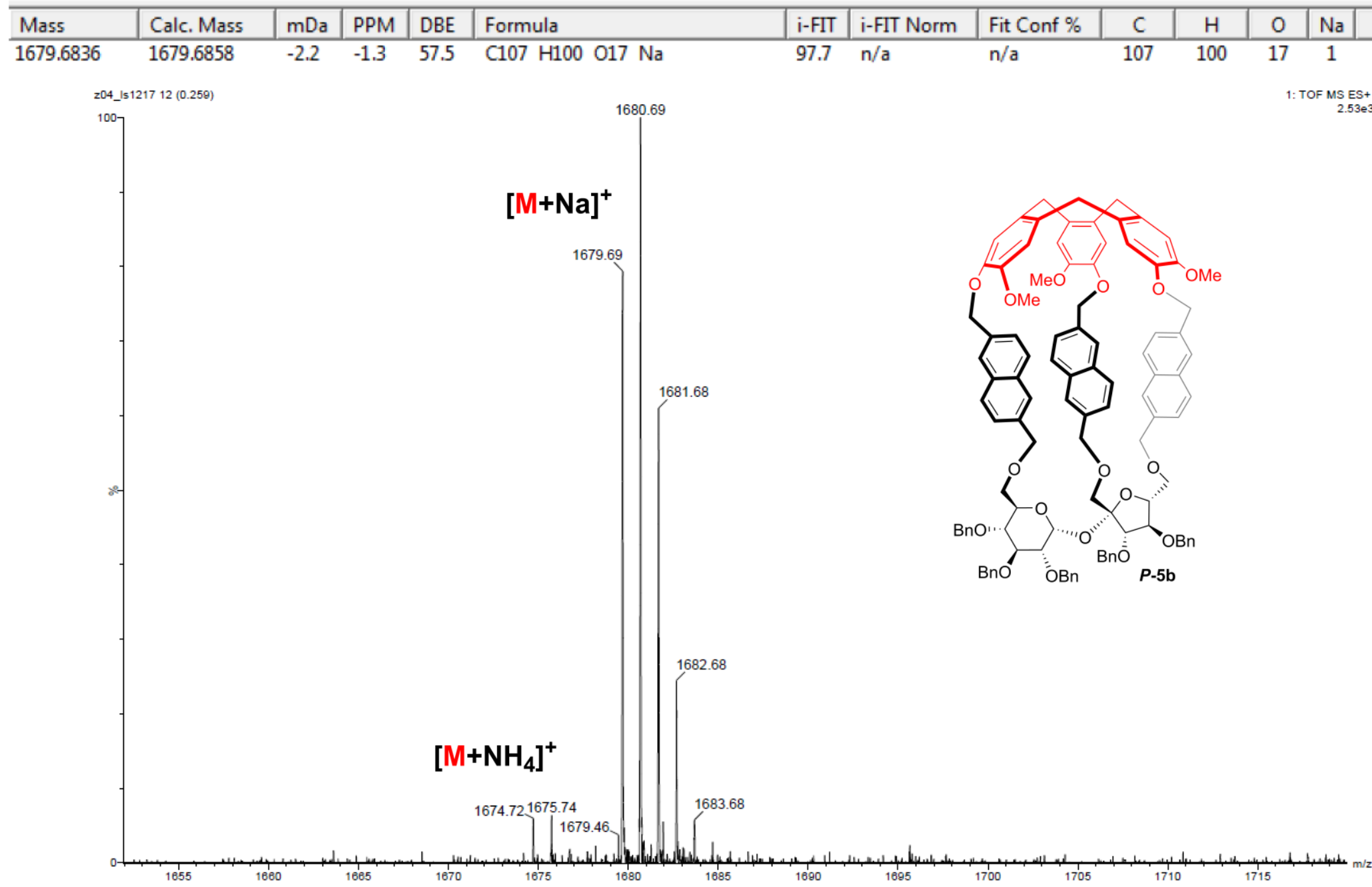

Figure S75. HRMS spectrum of compound $\boldsymbol{P}-\mathbf{5 b}$. 
Elements Used:
C. $0-150$
H: 0-150
0: $0-17$
Na: 0-1

Mass

\begin{tabular}{|l|l|l|l|l|} 
Calc. Mass & $\mathrm{mDa}$ & PPM & DBE & Formula \\
\hline
\end{tabular}

$\begin{array}{lllllllll}1679.6882 & -2.2 & -1.3 & 60.5 & \mathrm{C} 109 \mathrm{H} 99 & 017\end{array}$

1679.6858

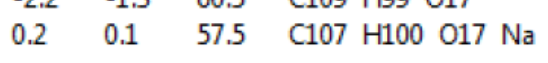

\begin{tabular}{|l|l|l}
\hline i-FIT & i-FIT Norm & Fit Conf \% \\
\hline
\end{tabular}

$154.8 \quad 1.985$

$152.9 \quad 0.148$

13.73

13.73
86.27

\begin{tabular}{c|c|c|c}
$\mathrm{C}$ & $\mathrm{H}$ & $\mathrm{O}$ & $\mathrm{Na}$ \\
109 & 99 & 17 & \\
107 & 100 & 17 & 1 \\
& & \multicolumn{1}{|c}{$1:$ TOF MS ES+ }
\end{tabular}

204_Is $101711(0.24)$

${ }^{204}{ }^{100}$ 1680.69

$280 \mathrm{e}$
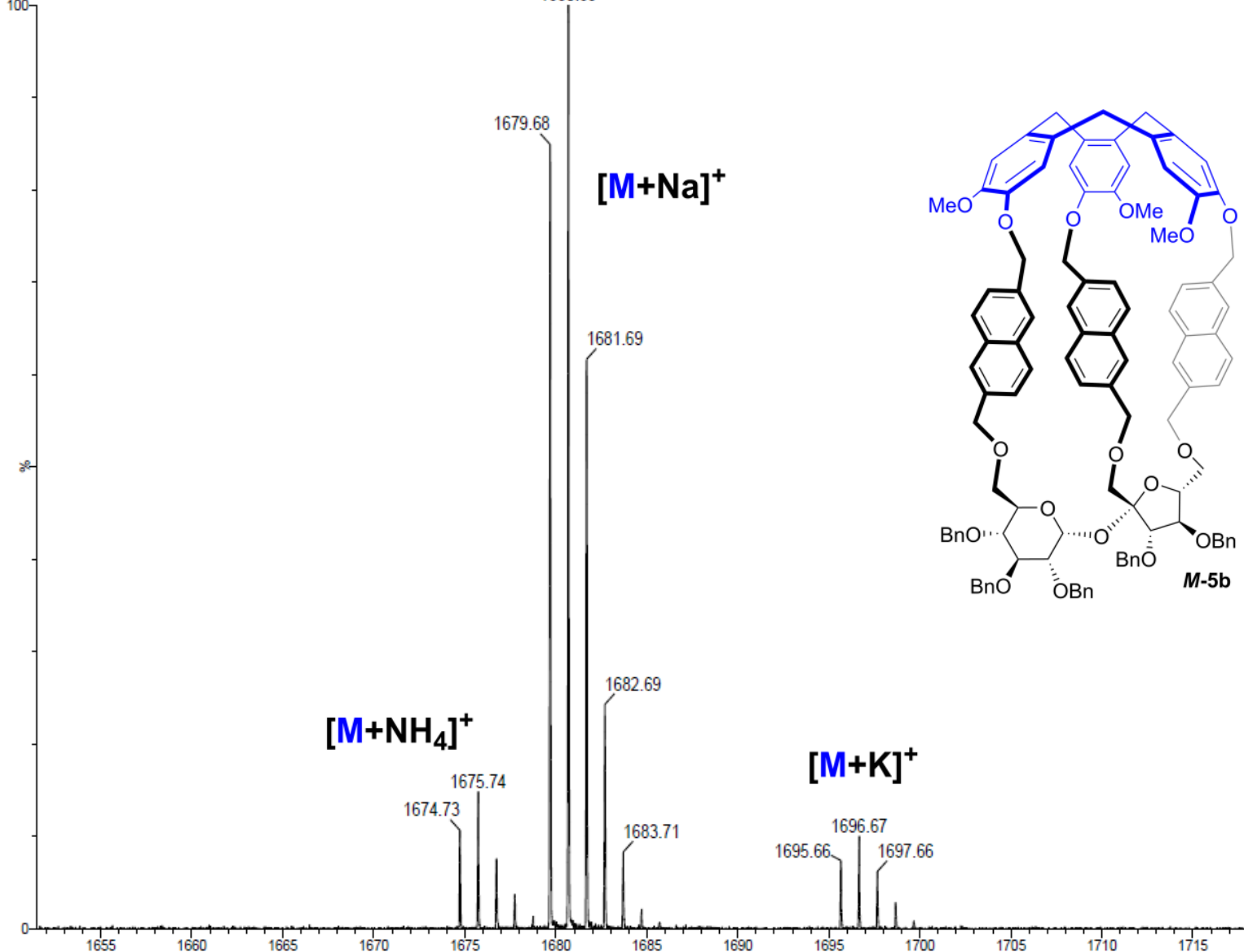

1665

1670

Figure S76. HRMS spectrum of compound $\boldsymbol{M}-\mathbf{5 b}$. 
KN1_Ch

z04_/151786 $20(0.415) \mathrm{Cm}(17: 28-(4: 14+34: 40)$

${ }^{100}$

1761.81

1: TOF MS ES+

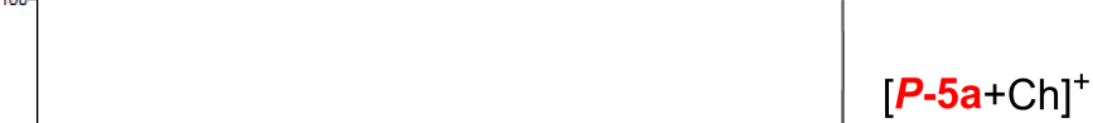

$[P-5 a+C h]^{+}$

1760.81

1762.82
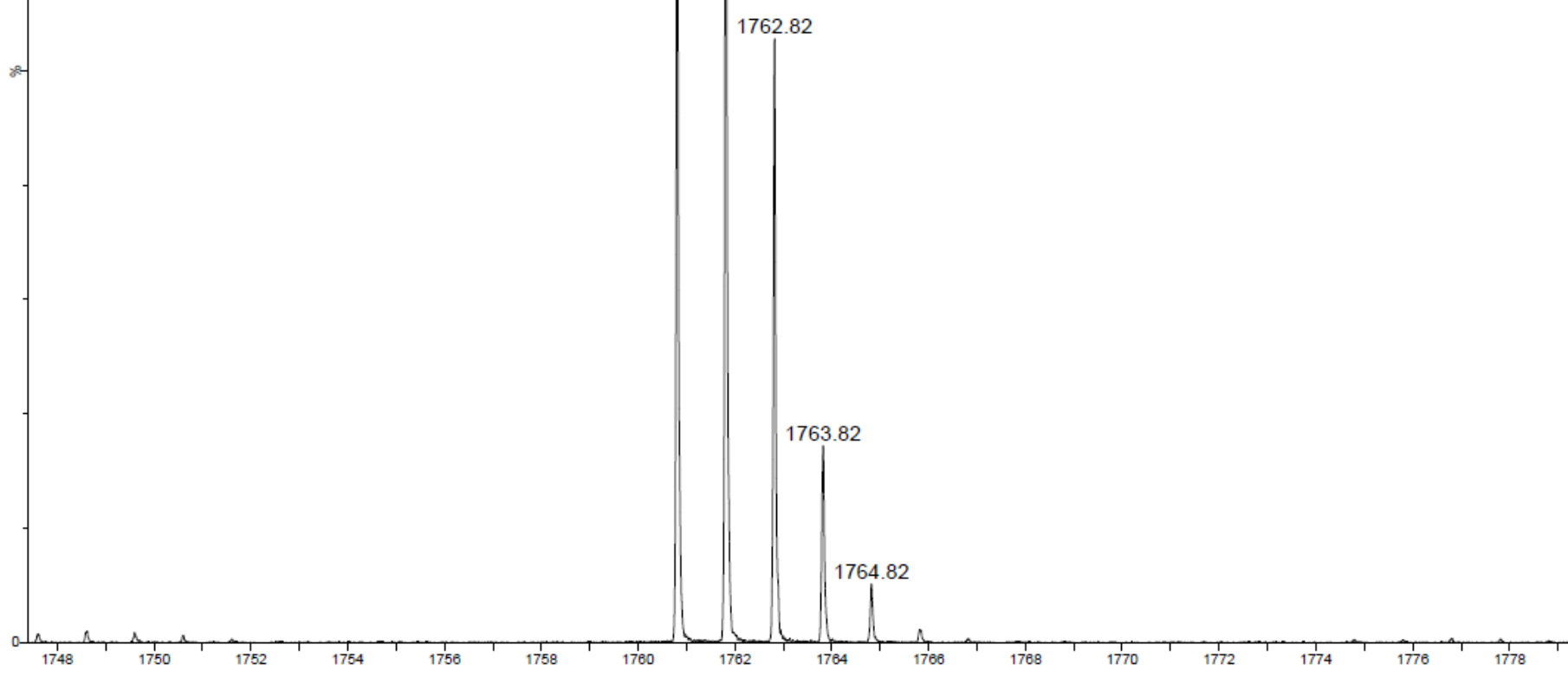

Figure S77. ESI-MS spectrum of adduct $[\boldsymbol{P}-5 \mathbf{a}+\mathrm{Ch}]^{+}$ 


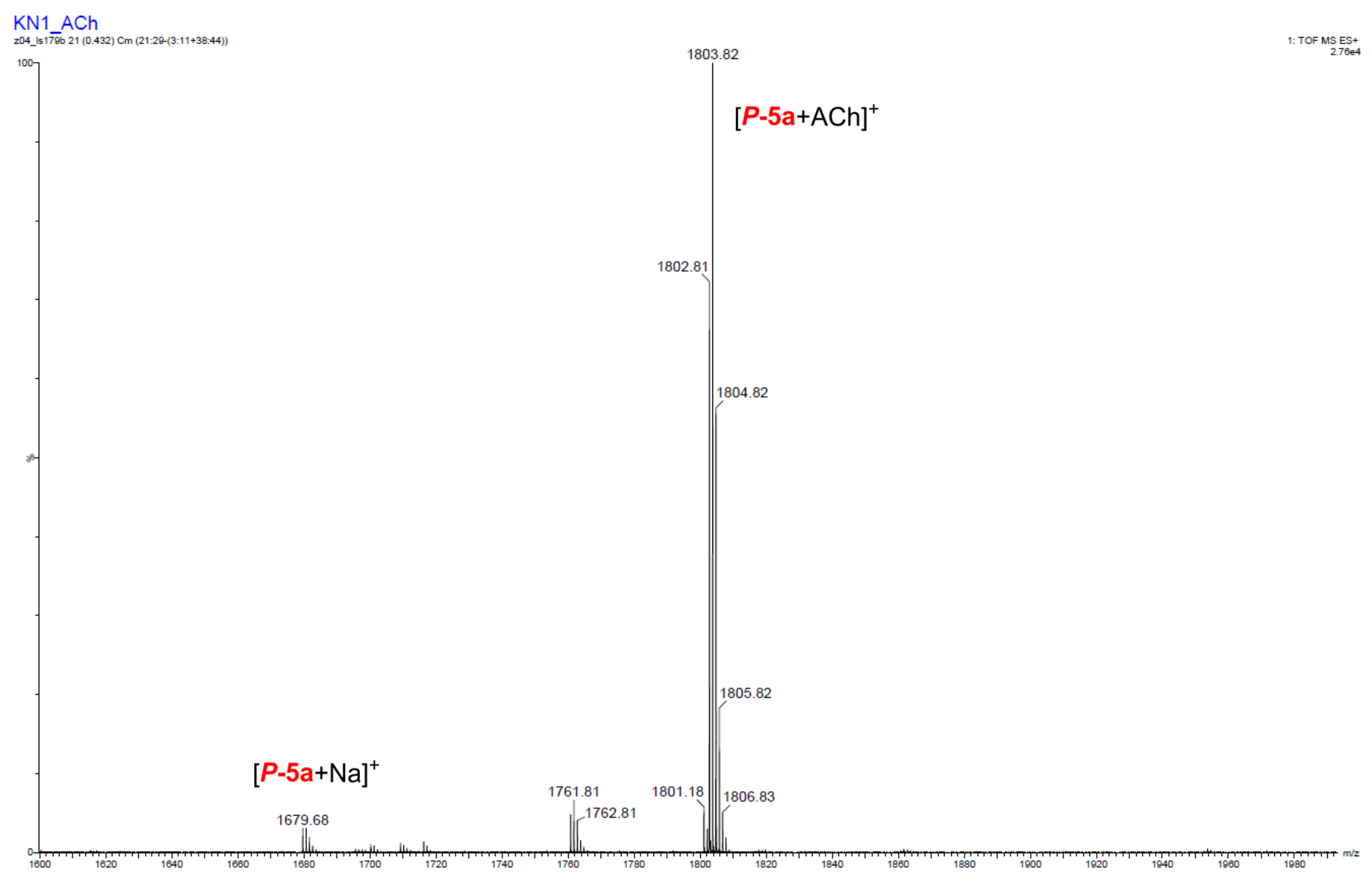

Figure S78. ESI-MS spectrum of adduct $[\mathbf{P}-5 \mathbf{a}+\mathrm{ACh}]^{+}$. 


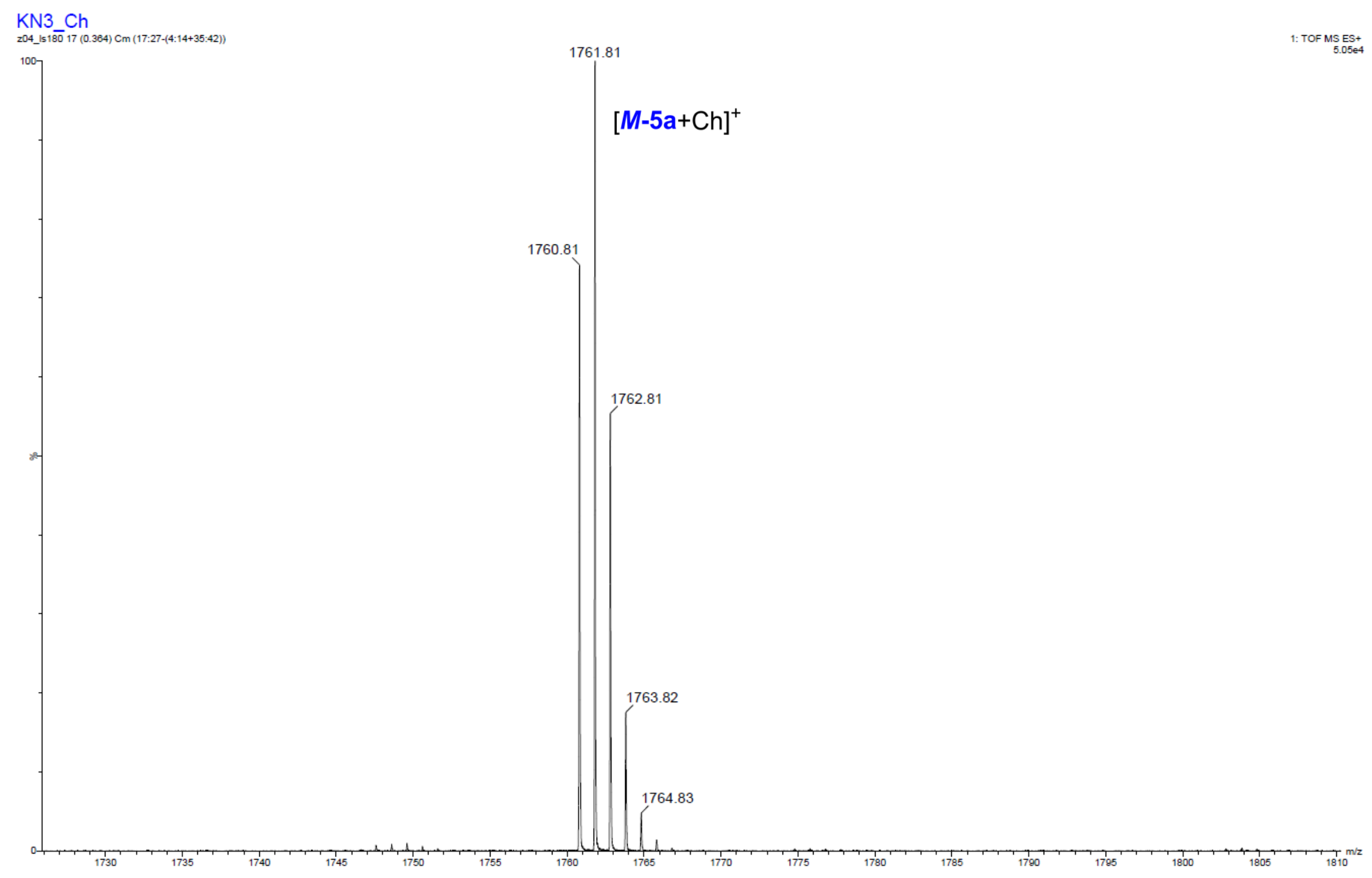

Figure S79. ESI-MS spectrum of adduct $[\mathbf{M}-5 \mathrm{a}+\mathrm{Ch}]^{+}$. 


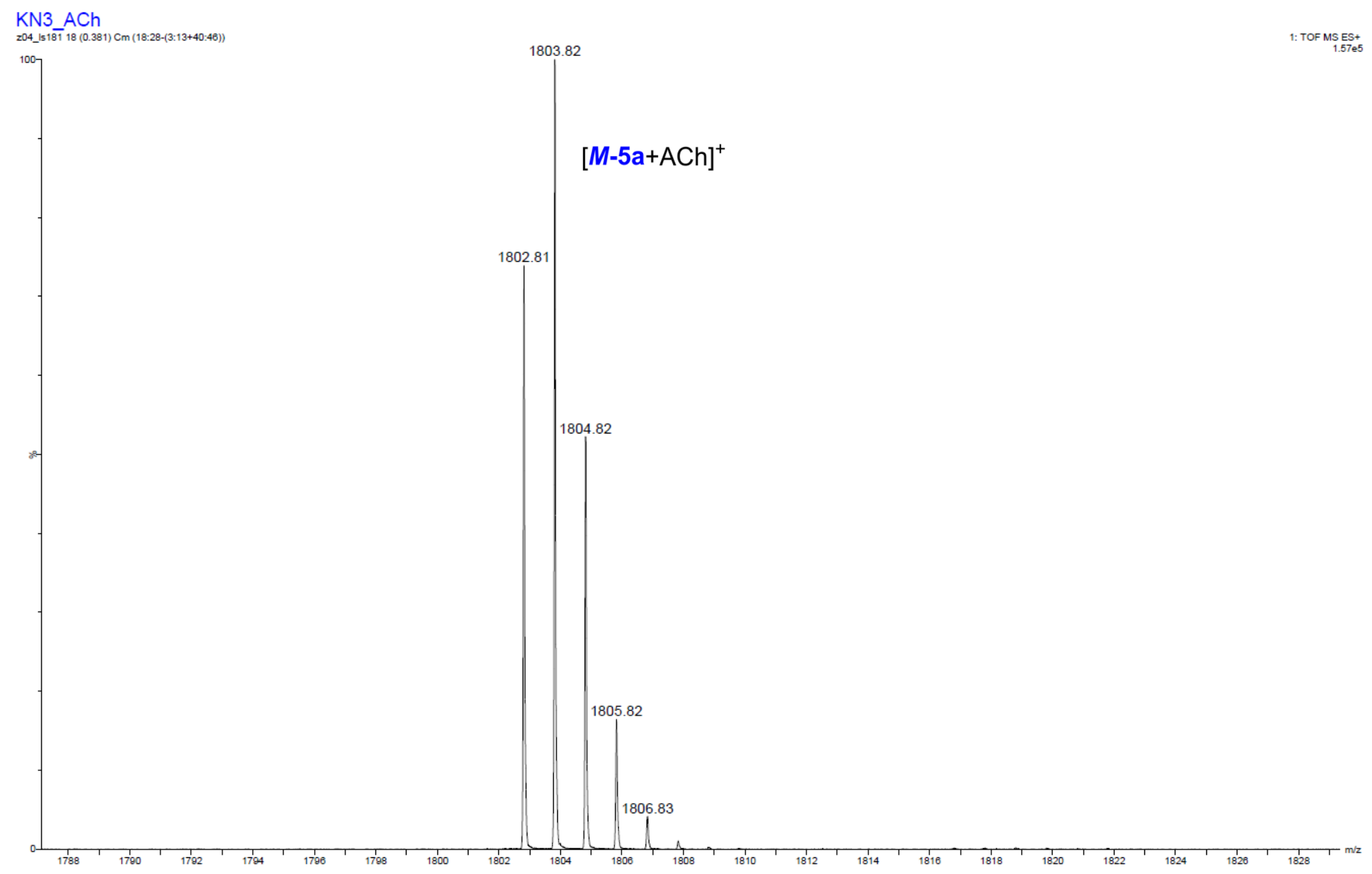

Figure S80. ESI-MS spectrum of adduct $[\mathbf{M}-\mathbf{5 a}+\mathrm{ACh}]^{+}$. 


\section{Geometry of calculated structures}

\section{P-5a}

Conformation \#1 (Pop. = 68.98\%)

Symbolic Z-matrix:

\begin{tabular}{|c|c|c|c|}
\hline C & 7.04048300 & 2.16352500 & 0.03090500 \\
\hline C & 7.39308900 & 1.65453100 & -1.36290000 \\
\hline C & 6.15137000 & 1.04199700 & -2.03362000 \\
\hline C & 4.70910400 & 2.52736100 & -0.81529100 \\
\hline C & 5.88632200 & 3.15908300 & -0.04844500 \\
\hline $\mathrm{O}$ & 5.14509900 & 2.03469400 & -2.08835300 \\
\hline $\mathrm{O}$ & 8.23163000 & 2.74531400 & 0.55846900 \\
\hline $\mathrm{O}$ & 8.43143400 & 0.68181500 & -1.29949700 \\
\hline $\mathrm{O}$ & 5.76710000 & -0.09268500 & -1.27973100 \\
\hline $\mathrm{O}$ & 5.52997300 & 3.51344000 & 1.28554900 \\
\hline C & 3.59479100 & 3.52440700 & -1.12896300 \\
\hline $\mathrm{O}$ & 3.04611100 & 3.96104700 & 0.11556800 \\
\hline C & 5.78723300 & -3.21146000 & -0.47286000 \\
\hline C & 7.13560800 & -2.82310900 & -1.06694500 \\
\hline C & 5.42737000 & -1.34221700 & -1.89827700 \\
\hline C & 6.69760500 & -2.11835900 & -2.35525100 \\
\hline $\mathrm{O}$ & 4.90001800 & -2.12649200 & -0.83860900 \\
\hline $\mathrm{O}$ & 7.69496700 & -1.36726700 & -3.00401900 \\
\hline C & 4.38803900 & -1.21957600 & -3.03351500 \\
\hline C & 5.72144800 & -3.45721300 & 1.02269700 \\
\hline $\mathrm{O}$ & 3.37239700 & -0.25443300 & -2.86726100 \\
\hline $\mathrm{H}$ & 7.71678400 & 2.50380400 & -1.98078800 \\
\hline $\mathrm{H}$ & 6.38495900 & -2.89239500 & -3.06824900 \\
\hline $\mathrm{H}$ & 7.64124500 & -2.10057000 & -0.41267500 \\
\hline $\mathrm{H}$ & 5.44250100 & -4.13044600 & -0.96944500 \\
\hline $\mathrm{H}$ & 6.72932200 & 1.31028400 & 0.64925800 \\
\hline $\mathrm{H}$ & 6.23206700 & 4.04788700 & -0.60103500 \\
\hline $\mathrm{H}$ & 4.29579100 & 1.70708400 & -0.21597800 \\
\hline $\mathrm{H}$ & 3.99499700 & 4.38130200 & -1.69133600 \\
\hline $\mathrm{H}$ & 2.83073200 & 3.03592700 & -1.74565100 \\
\hline $\mathrm{H}$ & 3.97809400 & -2.22910400 & -3.18777100 \\
\hline $\mathrm{H}$ & 4.66352700 & -3.50728800 & 1.31666400 \\
\hline $\mathrm{H}$ & 4.90559400 & -0.92962500 & -3.95451600 \\
\hline C & -3.91871600 & -4.22891900 & 1.07533900 \\
\hline C & -4.24732300 & -3.31916200 & 2.08119500 \\
\hline C & -4.74506300 & -4.30039100 & -0.06495900 \\
\hline C & -5.36360800 & -2.46839500 & 1.99093700 \\
\hline $\mathrm{H}$ & -3.62937800 & -3.26423800 & 2.97038700 \\
\hline C & -5.86599600 & -3.48308500 & -0.14066100 \\
\hline C & -6.19771800 & -2.55626300 & 0.86226700 \\
\hline $\mathrm{H}$ & -6.51555400 & -3.58276000 & -1.00598700 \\
\hline C & -2.93528500 & 1.13594000 & 3.72625400 \\
\hline C & -3.60205000 & 2.32731800 & 3.36573000 \\
\hline C & -3.61897300 & -0.07222200 & 3.62803600 \\
\hline C & -4.88042900 & 2.23454600 & 2.81708500 \\
\hline C & -4.92811100 & -0.16774500 & 3.12797800 \\
\hline $\mathrm{H}$ & -3.10086600 & -0.96407800 & 3.97056600 \\
\hline C & -5.54983300 & 1.00862300 & 2.67200300 \\
\hline $\mathrm{H}$ & -5.39399900 & 3.14048700 & 2.51561900 \\
\hline C & -6.49849900 & 1.81162900 & -1.71158100 \\
\hline
\end{tabular}




\begin{tabular}{|c|c|c|c|}
\hline C & -6.87819400 & 0.59694300 & -2.32822800 \\
\hline C & -6.53004600 & 1.89873800 & -0.32563100 \\
\hline C & -7.18659700 & -0.49533400 & -1.52112800 \\
\hline C & -6.87856000 & 0.81174100 & 0.49627200 \\
\hline $\mathrm{H}$ & -6.28044700 & 2.85868100 & 0.11780300 \\
\hline C & -7.17278600 & -0.41695400 & -0.11614900 \\
\hline $\mathrm{O}$ & -6.92835600 & 0.59662200 & -3.69387500 \\
\hline O & -2.94794100 & 3.50327900 & 3.60478400 \\
\hline C & -7.35670600 & -0.59186100 & -4.35330400 \\
\hline $\mathrm{H}$ & -7.34630400 & -0.36000700 & -5.41920600 \\
\hline $\mathrm{H}$ & -6.67641300 & -1.42931200 & -4.15640600 \\
\hline $\mathrm{H}$ & -8.37247400 & -0.87142300 & -4.04914000 \\
\hline C & -3.57219200 & 4.71924800 & 3.20152600 \\
\hline $\mathrm{H}$ & -3.75084500 & 4.73712900 & 2.11982100 \\
\hline $\mathrm{H}$ & -4.52002000 & 4.87617500 & 3.73015900 \\
\hline $\mathrm{H}$ & -2.87314700 & 5.51318400 & 3.46805700 \\
\hline C & -5.62458900 & -1.52806000 & 3.16474400 \\
\hline $\mathrm{H}$ & -5.29029600 & -2.03887000 & 4.07489800 \\
\hline $\mathrm{H}$ & -6.70021700 & -1.38238800 & 3.29137700 \\
\hline C & -6.91916500 & 1.03571200 & 2.00665600 \\
\hline $\mathrm{H}$ & -7.36665100 & 2.01945900 & 2.18848300 \\
\hline $\mathrm{H}$ & -7.59071000 & 0.31441900 & 2.47754300 \\
\hline $\mathrm{H}$ & -7.46399600 & -1.43645000 & -1.98242100 \\
\hline C & -7.44697200 & -1.70258000 & 0.65703000 \\
\hline $\mathrm{H}$ & -8.17342600 & -2.29611800 & 0.09055700 \\
\hline $\mathrm{H}$ & -7.92378000 & -1.48543900 & 1.61531100 \\
\hline $\mathrm{H}$ & 6.35759800 & 0.78008800 & -3.07191800 \\
\hline $\mathrm{H}$ & 4.67478000 & 3.98218300 & 1.22328100 \\
\hline $\mathrm{H}$ & 9.09858900 & 1.01390700 & -0.67391100 \\
\hline $\mathrm{H}$ & 8.08963200 & -0.74649600 & -2.35320900 \\
\hline $\mathrm{H}$ & 8.04976200 & 2.98128600 & 1.48271600 \\
\hline $\mathrm{O}$ & -2.85708800 & -5.08452700 & 1.11457900 \\
\hline C & -1.99695400 & -5.03240400 & 2.24872000 \\
\hline $\mathrm{H}$ & -1.53224700 & -4.04458000 & 2.35486100 \\
\hline $\mathrm{H}$ & -2.53444300 & -5.28125100 & 3.17203500 \\
\hline $\mathrm{H}$ & -1.22269800 & -5.77975400 & 2.06864100 \\
\hline $\mathrm{O}$ & 6.40144200 & -2.43619800 & 1.73430800 \\
\hline $\mathrm{H}$ & 6.17509900 & -4.43805600 & 1.23680900 \\
\hline O & -6.21349100 & 2.93729400 & -2.45149000 \\
\hline $\mathrm{O}$ & -1.66539800 & 1.11615200 & 4.25930500 \\
\hline C & -0.66778500 & 1.92290100 & 3.61979200 \\
\hline $\mathrm{H}$ & -0.59496200 & 2.88821600 & 4.13264500 \\
\hline $\mathrm{H}$ & -0.97008300 & 2.13412200 & 2.58652700 \\
\hline $\mathrm{O}$ & 7.93010200 & -3.97890100 & -1.27194100 \\
\hline $\mathrm{H}$ & 8.75219300 & -3.68481400 & -1.69768600 \\
\hline C & 1.97535100 & 4.90032200 & -0.00080400 \\
\hline $\mathrm{H}$ & 2.28806800 & 5.75900200 & -0.61169400 \\
\hline $\mathrm{H}$ & 1.81705900 & 5.26091800 & 1.02167700 \\
\hline C & 0.69146500 & 4.30565200 & -0.55131900 \\
\hline $\mathrm{C}$ & -0.17740000 & 5.08306200 & -1.28909700 \\
\hline C & 0.34388000 & 2.95529100 & -0.26839800 \\
\hline C & -1.41320400 & 4.56665200 & -1.76549600 \\
\hline $\mathrm{H}$ & 0.07569800 & 6.11664600 & -1.51802500 \\
\hline C & -0.83290100 & 2.41575700 & -0.73773400 \\
\hline $\mathrm{H}$ & 1.02802400 & 2.34849400 & 0.31666000 \\
\hline $\mathrm{C}$ & -2.32711200 & 5.35410300 & -2.51699300 \\
\hline C & -1.74379000 & 3.19776800 & -1.50123500 \\
\hline $\mathrm{H}$ & -1.07783300 & 1.37706400 & -0.53057200 \\
\hline
\end{tabular}




\begin{tabular}{|c|c|c|c|}
\hline & -3.49646700 & 4.80922200 & -2.99652 \\
\hline & -2.08388500 & 6.39485400 & -2.7165560 \\
\hline & -2.95075500 & 2.66449200 & -2.0275 \\
\hline & -3.82013800 & 3.44311000 & -2.7691 \\
\hline & -4.18077600 & 5.42298800 & -3.57739 \\
\hline & -3.17610800 & 1.61484400 & $-1.8505 t$ \\
\hline & 2.50701500 & -0.38592400 & -1.7275 \\
\hline & 2.05328900 & 0.60415000 & $-1.62661 C$ \\
\hline & 3.09250900 & -0.58638700 & -0.826120 \\
\hline & 1.40888900 & -1.41854600 & -1.878917 \\
\hline & 0.21130000 & -1.07749200 & -2.4779 \\
\hline & 1.56768000 & -2.73157000 & -1.35241 \\
\hline & -0.87563700 & -1.98899000 & -2.53996 \\
\hline & 0.07253600 & -0.07514500 & -2.878555 \\
\hline & 0.53885000 & -3.64391400 & -1.403397 \\
\hline & 2.51234200 & -2.99460700 & -0.886303 \\
\hline & -2.13529500 & -1.63648900 & -3.0948 \\
\hline & -0.71648600 & -3.29664000 & -1.97398 \\
\hline & 0.66462100 & -4.63799400 & -0.980748 \\
\hline & -3.19368400 & -2.51671000 & -3.052656 \\
\hline & -2.26039700 & -0.64778600 & -3.52988 \\
\hline & -1.82460000 & -4.18128700 & -1.96023 \\
\hline & -3.05202300 & -3.80362700 & -2.46887 \\
\hline & -4.15874900 & -2.22106000 & -3.456693 \\
\hline & -1.71160000 & -5.15522900 & -1.49255 \\
\hline & 6.10148700 & -2.43140000 & 3.135214 \\
\hline & 6.89726500 & -1.83392500 & 3.590888 \\
\hline & 6.16689000 & -3.45035300 & 3.543512 \\
\hline & 4.74497900 & -1.82073300 & 3.42546 \\
\hline & 4.53009200 & -0.48654700 & 3.136339 \\
\hline & 3.66845100 & -2.59347500 & 3.934017 \\
\hline & 3.26199500 & 0.11604200 & 3.315155 \\
\hline & 5.34508300 & 0.12071900 & 2.748030 \\
\hline & 2.41928200 & -2.03745800 & 4.121935 \\
\hline & 3.83488200 & -3.64154800 & 4.172263 \\
\hline & 3.03102700 & 1.48511200 & 3.004588 \\
\hline & 2.17250800 & -0.67499900 & 3.806543 \\
\hline & 1.60211400 & -2.64543800 & 4.502756 \\
\hline & 1.77997400 & 2.02967200 & 3.152874 \\
\hline & 3.85628800 & 2.08616900 & 2.631242 \\
\hline & 0.88466200 & -0.07852400 & 3.934426 \\
\hline & 0.68079000 & 1.24226800 & 3.603335 \\
\hline & 1.61501500 & 3.07676300 & 2.909769 \\
\hline & 0.05263500 & -0.68293200 & 4.282707 \\
\hline & -5.08237300 & 2.86100900 & -3.361735 \\
\hline & -5.38510600 & 3.43452300 & -4.242319 \\
\hline & -4.92797500 & 1.82501900 & -3.666970 \\
\hline & -4.44770300 & -5.23859400 & -1.033766 \\
\hline & -4.24212400 & -4.73543700 & -2.369168 \\
\hline & -4.09311600 & -5.63889200 & $-2.96847 ?$ \\
\hline & -5.14748200 & -4.23773200 & $-2.73685 c$ \\
\hline
\end{tabular}

Imaginary Frequency $=0$

$E(R B 3 L Y P)=-4064.5336189$ a.u. 
Conformation \#2 (Pop. = 20.28\%)

Symbolic Z-matrix:

$\begin{array}{lrrr}\text { C } & -6.57531800 & -2.47382400 & -0.12229800 \\ \text { C } & -7.20312100 & -1.92903900 & -1.39453600 \\ \text { C } & -6.28117300 & -0.87372100 & -2.02995600 \\ \text { C } & -4.34046600 & -2.01955400 & -1.16166700 \\ \text { C } & -5.20835700 & -3.06896200 & -0.43508500 \\ \text { O } & -5.02548300 & -1.47830400 & -2.30487900 \\ \text { O } & -7.48712800 & -3.44142900 & 0.39667000 \\ \text { O } & -8.48104800 & -1.36226500 & -1.12405100 \\ \text { O } & -6.15239100 & 0.22317400 & -1.15447500 \\ \text { O } & -4.64187800 & -3.47641600 & 0.80228200 \\ \text { C } & -3.04690900 & -2.61804200 & -1.71547700 \\ \text { O } & -2.29699200 & -3.10713300 & -0.60188300 \\ \text { C } & -6.97461500 & 2.84635600 & 0.27404700 \\ \text { C } & -8.21068900 & 2.12200000 & -0.25535600 \\ \text { C } & -6.31873100 & 1.58936900 & -1.61865300 \\ \text { C } & -7.83355600 & 1.92176100 & -1.73031800 \\ \text { O } & -5.87696600 & 2.39970600 & -0.56019700 \\ \text { O } & -8.64237000 & 1.02461500 & -2.45081000 \\ \text { C } & -5.48329000 & 1.92358500 & -2.88205500 \\ \text { C } & -6.62774400 & 2.66676300 & 1.74087000 \\ \text { O } & -4.19768300 & 2.45299900 & -2.62465100 \\ \text { H } & -7.31012500 & -2.74750500 & -2.12031000 \\ \text { H } & -7.92711000 & 2.89303000 & -2.23111300 \\ \text { H } & -8.32232800 & 1.14387000 & 0.22837600 \\ \text { H } & -7.11480700 & 3.92355800 & 0.10592200 \\ \text { H } & -6.44520000 & -1.65208500 & 0.59488400 \\ \text { H } & -5.34834300 & -3.93408100 & -1.10518600 \\ \text { H } & -4.09833900 & -1.21821600 & -0.45221100 \\ \text { H } & -3.28308800 & -3.43998500 & -2.40680100 \\ \text { H } & -2.48184500 & -1.85554600 & -2.26585900 \\ \text { H } & -6.00603500 & 2.71230300 & -3.43058400 \\ \text { H } & -5.68513000 & 3.19641400 & 1.93641100 \\ \text { H } & -5.42118000 & 1.04638000 & -3.54001900 \\ \mathrm{C} & 3.58460100 & 3.46648200 & 2.48785000 \\ \mathrm{C} & 4.03641000 & 2.31871600 & 3.14065900 \\ \mathrm{C} & 4.38391700 & 4.01711700 & 1.46490200 \\ \mathrm{C} & 5.25025300 & 1.69181100 & 2.80641600 \\ \mathrm{C} & 3.43759400 & 1.88917100 & 3.93578400 \\ \mathrm{C} & 5.60154300 & 3.42097800 & 1.16177700 \\ \mathrm{C} & 7.33453600 & 1.35746100 & -1.11598400 \\ \mathrm{C} & & & \end{array}$




\begin{tabular}{|c|c|c|c|}
\hline C & 7.13222500 & -0.63355900 & 0.25304000 \\
\hline $\mathrm{H}$ & 6.83108600 & -2.44374900 & -0.89651600 \\
\hline C & 7.29349500 & 0.75850500 & 0.15634300 \\
\hline 0 & 7.26540000 & 1.13820300 & -3.55073300 \\
\hline $\mathrm{O}$ & 3.29327400 & -4.49928800 & 1.84366700 \\
\hline C & 7.48444400 & 2.53951400 & -3.69197500 \\
\hline $\mathrm{H}$ & 7.50830400 & 2.72922800 & -4.76593900 \\
\hline $\mathrm{H}$ & 6.67262400 & 3.11965500 & -3.23640700 \\
\hline $\mathrm{H}$ & 8.44018100 & 2.84055100 & -3.24658900 \\
\hline C & 3.96012600 & -5.37204700 & 0.93568300 \\
\hline $\mathrm{H}$ & 4.11831200 & -4.89084400 & -0.03699200 \\
\hline $\mathrm{H}$ & 4.92347600 & -5.70834500 & 1.33761000 \\
\hline $\mathrm{H}$ & 3.30028600 & -6.23218100 & 0.81319800 \\
\hline C & 5.62440700 & 0.42856300 & 3.57651100 \\
\hline $\mathrm{H}$ & 5.24844500 & 0.53724400 & 4.60014700 \\
\hline $\mathrm{H}$ & 6.71015500 & 0.35069900 & 3.66899600 \\
\hline C & 7.13809500 & -1.39754600 & 1.57548200 \\
\hline $\mathrm{H}$ & 7.66036300 & -2.34680900 & 1.41014900 \\
\hline $\mathrm{H}$ & 7.73199100 & -0.85994400 & 2.31810900 \\
\hline $\mathrm{H}$ & 7.50177900 & 2.42686300 & -1.17721600 \\
\hline C & 7.40049200 & 1.68184300 & 1.36539100 \\
\hline $\mathrm{H}$ & 8.05709600 & 2.51811900 & 1.09992300 \\
\hline $\mathrm{H}$ & 7.89013600 & 1.17295500 & 2.19833600 \\
\hline $\mathrm{H}$ & -6.67314900 & -0.56892100 & -3.00188900 \\
\hline $\mathrm{H}$ & -3.68856600 & -3.61192800 & 0.63947700 \\
\hline $\mathrm{H}$ & -8.91992000 & -1.94440600 & -0.47895200 \\
\hline $\mathrm{H}$ & -8.73750300 & 0.20216000 & -1.92272400 \\
\hline $\mathrm{H}$ & -7.13014900 & -3.73718500 & 1.25009700 \\
\hline $\mathrm{O}$ & 2.42356900 & 4.12170800 & 2.77670200 \\
\hline C & 1.58421000 & 3.58109400 & 3.79263800 \\
\hline $\mathrm{H}$ & 1.24861900 & 2.56795600 & 3.53885200 \\
\hline $\mathrm{H}$ & 2.09166600 & 3.56169200 & 4.76488200 \\
\hline $\mathrm{H}$ & 0.72085800 & 4.24579800 & 3.84896300 \\
\hline $\mathrm{O}$ & -6.52149200 & 1.29132500 & 2.06696900 \\
\hline $\mathrm{H}$ & -7.41261300 & 3.15428000 & 2.34183900 \\
\hline O & 6.86909600 & -1.56495000 & -3.31441300 \\
\hline $\mathrm{O}$ & 1.90084900 & -2.71508500 & 3.46212600 \\
\hline C & 0.90668100 & -3.16362600 & 2.50868600 \\
\hline $\mathrm{H}$ & 0.84845900 & -4.25517500 & 2.53992800 \\
\hline $\mathrm{H}$ & 1.21940100 & -2.86854200 & 1.50253100 \\
\hline $\mathrm{O}$ & -9.36068200 & 2.92864600 & -0.05139600 \\
\hline $\mathrm{H}$ & -10.11382700 & 2.44790500 & -0.43223200 \\
\hline C & -1.20169600 & -3.96076500 & -0.92737400 \\
\hline $\mathrm{H}$ & -1.52991800 & -4.75172500 & -1.61681300 \\
\hline $\mathrm{H}$ & -0.93953300 & -4.44043400 & 0.02239800 \\
\hline C & 0.00992100 & -3.24088700 & -1.49103700 \\
\hline C & 0.93919500 & -3.93990500 & -2.23578500 \\
\hline C & 0.24184900 & -1.86947200 & -1.19983200 \\
\hline C & 2.12999700 & -3.32266000 & -2.70117700 \\
\hline $\mathrm{H}$ & 0.77330800 & -4.99038800 & -2.46817700 \\
\hline C & 1.37232800 & -1.23300100 & -1.66288700 \\
\hline $\mathrm{H}$ & -0.48966000 & -1.32571300 & -0.61040700 \\
\hline C & 3.11604800 & -4.03114100 & -3.44349100 \\
\hline C & 2.34764200 & -1.93447000 & -2.42176600 \\
\hline $\mathrm{H}$ & 1.53283700 & -0.17975900 & -1.44728200 \\
\hline C & 4.25091500 & -3.39581100 & -3.89085900 \\
\hline $\mathrm{H}$ & 2.95562000 & -5.08515800 & -3.65702100 \\
\hline C & 3.52334200 & -1.30751300 & -2.91642200 \\
\hline
\end{tabular}




\begin{tabular}{|c|c|c|c|}
\hline & 4.46555100 & -2.01105000 & -3.64083400 \\
\hline & 4.99436900 & -3.95008200 & -4.45875700 \\
\hline & 3.66918500 & -0.24770000 & -2.718535 \\
\hline & -3.23000200 & 1.51827300 & -2.136276 \\
\hline & -2.98509800 & 0.78329700 & -2.916910 \\
\hline & -3.63797700 & 0.97583200 & -1.275647 \\
\hline & -2.00729200 & 2.29391100 & -1.7165710 \\
\hline & -0.79268000 & 2.14132600 & $-2.349647 C$ \\
\hline & -2.11756400 & 3.20911200 & -0.630173 \\
\hline & 0.35690100 & 2.86889100 & -1.930357 \\
\hline & -0.69701500 & 1.45145500 & -3.185528 \\
\hline & -1.02770500 & 3.92432600 & $-0.196391 c$ \\
\hline & -3.08447600 & 3.33005900 & -0.1504770 \\
\hline & 1.62041000 & 2.73188600 & -2.5644910 \\
\hline & 0.24105200 & 3.77507700 & -0.826374 \\
\hline & -1.11977100 & 4.61731300 & 0.636592 \\
\hline & 2.71366700 & 3.44587300 & -2.121700 \\
\hline & 1.71683100 & 2.04771600 & -3.4041200 \\
\hline & 1.38495900 & 4.49482400 & $-0.398930 c$ \\
\hline & 2.60699300 & 4.34109300 & -1.025307 \\
\hline & 3.67505600 & 3.32178400 & -2.614668 \\
\hline & 1.29799900 & 5.16121400 & 0.454046 \\
\hline & -5.93850300 & 1.01752400 & 3.3505750 \\
\hline & -6.58862400 & 0.29251400 & 3.8526110 \\
\hline & -5.92876500 & 1.93134900 & 3.9590660 \\
\hline & -4.54349600 & 0.44585600 & 3.212644 \\
\hline & -4.29837500 & -0.88463900 & 3.481305 \\
\hline & -3.46715700 & 1.26659200 & 2.763918 \\
\hline & -3.00766600 & -1.45641200 & 3.305796 \\
\hline & -5.10732100 & -1.52670300 & 3.823555 \\
\hline & -2.20848900 & 0.74832200 & 2.577522 \\
\hline & -3.64464100 & 2.32046300 & 2.569306 \\
\hline & -2.74295200 & -2.82828100 & 3.556429 \\
\hline & -1.94004600 & -0.62651500 & $2.832439 c$ \\
\hline & -1.40143600 & 1.38497200 & 2.2234380 \\
\hline & -1.49124500 & -3.35775400 & 3.326464 \\
\hline & -3.54798000 & -3.46098600 & 3.921105 \\
\hline & -0.66497200 & -1.20414900 & 2.608709 \\
\hline & -0.43362600 & -2.54900400 & 2.833449 \\
\hline & -1.30597300 & -4.41277300 & 3.5129610 \\
\hline & 0.13647600 & -0.57171400 & 2.232477 \\
\hline & 5.71079100 & -1.33656400 & -4.162231 \\
\hline & 6.00019100 & -1.75914100 & -5.128340 \\
\hline & 5.55478700 & -0.26289700 & -4.286370 \\
\hline & 3.96897800 & 5.18487500 & 0.855230 \\
\hline & 3.81498400 & 5.13891900 & -0.574142 \\
\hline & 3.71546000 & 6.18997500 & -0.86453 \\
\hline & 472383 & 4.75068 & \\
\hline
\end{tabular}

Imaginary Frequency $=0$

$E(R B 3 L Y P)=-4064.5324641$ a.u. 


\section{M-5a}

Conformation \#1 (Pop. = 81.87\%)

Symbolic Z-matrix:

\begin{tabular}{|c|c|c|c|}
\hline & -5.80016800 & -0.71927100 & 3.1 \\
\hline & -6.86809800 & -1.52981300 & 2. \\
\hline & -6.42566900 & -1.82120200 & 0.940434 \\
\hline & -4.11168000 & -1.81589900 & $1.59341 \mathrm{~s}$ \\
\hline & -4.46098100 & -1.45032300 & 3.04999 \\
\hline & -5.19618600 & -2.51798400 & 0.96911 \\
\hline & -6.26107600 & -0.53720000 & 4.44345 \\
\hline & -6.34247200 & -0.58743500 & 0.2562 \\
\hline & -2.92890400 & -2.77726700 & 1.49683 \\
\hline & -1.79427300 & -2.16753000 & 2.110239 \\
\hline & -7.42087800 & 1.81154100 & -1.506946 \\
\hline & -8.57397700 & 1.17992300 & -0.7334 \\
\hline & -6.84792800 & -0.44765300 & -1.0836 \\
\hline & -6.38044700 & 0.80581400 & -1.532250 \\
\hline & -6.33043800 & -1.52750200 & -2.033933 \\
\hline & -4.91984300 & -1.56355600 & -1.982207 \\
\hline & -6.98820900 & -2.49541900 & 2.893826 \\
\hline & -8.42902500 & 1.32204800 & 0.345375 \\
\hline & -7.75614300 & 1.99295300 & -2.538438 \\
\hline & -5.69366800 & 0.25353300 & 2.606193 \\
\hline & -4.55445800 & -2.38203900 & 3.632686 \\
\hline & -3.88748900 & -0.89682700 & 1.038414 \\
\hline & -3.18253400 & -3.71608800 & 2.012070 \\
\hline & -2.72080100 & -3.00695500 & 0.443625 \\
\hline & -6.68570300 & -1.27423000 & -3.044354 \\
\hline & -6.75682500 & -2.50833600 & -1.770546 \\
\hline & 4.97649600 & -3.41454300 & -1.451885 \\
\hline & 5.13722200 & -2.40565100 & -2.393259 \\
\hline & 5.80674100 & -3.42348100 & -0.309603 \\
\hline & 6.05895200 & -1.35711800 & -2.232924 \\
\hline & 4.51273900 & -2.45381500 & -3.280843 \\
\hline & 6.70790300 & -2.37558900 & -0.130780 \\
\hline & 6.83550900 & -1.32877600 & -1.061976 \\
\hline & 7.34762200 & -2.36837200 & 0.744655 \\
\hline & 2.77679100 & 1.45275400 & -3.608710 \\
\hline & 3.00315900 & 2.66765000 & -2.919072 \\
\hline & 3.82976500 & 0.55629800 & -3.745903 \\
\hline & 4.27747300 & 2.94405900 & -2.439975 \\
\hline & 5.10670500 & 0.80627100 & -3.204571 \\
\hline & 3.66600500 & -0.37742600 & -4.271610 \\
\hline & 5.34056800 & 2.02564300 & -2.554989 \\
\hline & 4.46345700 & 3.89963800 & -1.960718 \\
\hline & 6.22350900 & 2.95537800 & 1.816999 \\
\hline & 6.62532300 & 1.71405000 & 2.356444 \\
\hline & 6.27760300 & 3.13111800 & 0.434442 \\
\hline & 7.12829200 & 0.74447500 & 1.495799 \\
\hline & 6.74699200 & 2.13191100 & -0.436291 \\
\hline & 5.96382500 & 4.07759900 & 0.009114 \\
\hline & 7.21491100 & 0.92280200 & 0.105981 \\
\hline & 6.65234100 & 1.43635600 & 3.7045 \\
\hline & 19 & 3.4 & \\
\hline
\end{tabular}




\begin{tabular}{|c|c|c|c|}
\hline 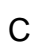 & 1.90294700 & 4.46718900 & -1.74229200 \\
\hline & 2.54295500 & 4.12489600 & -0.91905500 \\
\hline $\mathrm{H}$ & 2.30593700 & 5.42075000 & -2.10602900 \\
\hline C & 6.14576200 & -0.30220400 & -3.33355800 \\
\hline $\mathrm{H}$ & 5.98975500 & -0.80549300 & -4.29471300 \\
\hline $\mathrm{H}$ & 7.15345600 & 0.11639400 & -3.37901500 \\
\hline C & 6.67948200 & 2.41626800 & -1.93316700 \\
\hline $\mathrm{H}$ & 6.82532900 & 3.49195700 & -2.08524200 \\
\hline $\mathrm{H}$ & 7.50320400 & 1.92744400 & -2.45780200 \\
\hline $\mathrm{H}$ & 7.46905700 & -0.18298200 & 1.94752900 \\
\hline C & 7.81448100 & -0.20890900 & -0.72702600 \\
\hline $\mathrm{H}$ & 8.64209200 & -0.64241400 & -0.15386700 \\
\hline $\mathrm{H}$ & 8.26412200 & 0.19161000 & -1.63826600 \\
\hline $\mathrm{H}$ & -7.12895600 & -2.49920500 & 0.45555200 \\
\hline $\mathrm{H}$ & -5.60396800 & 0.01867200 & 4.89375200 \\
\hline 0 & 4.07414200 & -4.42097200 & -1.70642000 \\
\hline 0 & -6.57034600 & 2.99877400 & 0.41062400 \\
\hline 0 & -9.80567500 & 1.73618100 & -1.16340200 \\
\hline $\mathrm{H}$ & -10.50716400 & 1.29965800 & -0.65286500 \\
\hline C & 3.06497700 & -4.67528800 & -0.69399800 \\
\hline $\mathrm{H}$ & 3.19208100 & -5.70885100 & -0.35859000 \\
\hline $\mathrm{H}$ & 3.23319000 & -4.01880200 & 0.16218100 \\
\hline C & 5.46290800 & 1.66122700 & 4.50362300 \\
\hline $\mathrm{H}$ & 5.28361200 & 2.73309600 & 4.60398700 \\
\hline $\mathrm{H}$ & 5.74620800 & 1.25905000 & 5.48001100 \\
\hline 0 & 5.66776100 & -4.48900000 & 0.53492500 \\
\hline 0 & 5.82103900 & 3.91672600 & 2.70323500 \\
\hline 0 & 1.51175700 & 1.25994400 & -4.08571100 \\
\hline C & 1.25496300 & 0.07205800 & -4.82888400 \\
\hline $\mathrm{H}$ & 1.41063000 & -0.82594100 & -4.21891000 \\
\hline $\mathrm{H}$ & 0.20665200 & 0.12806800 & -5.12594500 \\
\hline $\mathrm{H}$ & 1.88647000 & 0.01700500 & -5.72416900 \\
\hline C & 6.45173600 & -4.51142700 & 1.72466500 \\
\hline $\mathrm{H}$ & 6.23640400 & -3.64456300 & 2.36098700 \\
\hline $\mathrm{H}$ & 6.17081900 & -5.42635000 & 2.24830700 \\
\hline $\mathrm{H}$ & 7.52407000 & -4.53753600 & 1.49642500 \\
\hline C & 5.41858000 & 5.18432600 & 2.18951900 \\
\hline $\mathrm{H}$ & 4.54955400 & 5.09011500 & 1.52744300 \\
\hline $\mathrm{H}$ & 5.14908700 & 5.78439600 & 3.05967300 \\
\hline $\mathrm{H}$ & 6.23774400 & 5.67255500 & 1.64839400 \\
\hline C & -6.87247500 & 3.12158200 & -0.97090000 \\
\hline $\mathrm{H}$ & -7.62408700 & 3.91081400 & -1.13139900 \\
\hline $\mathrm{H}$ & -5.97588600 & 3.38742000 & -1.54635100 \\
\hline C & -0.67801000 & -3.04780200 & 2.19136500 \\
\hline $\mathrm{H}$ & -0.91391000 & -3.89156700 & 2.85821300 \\
\hline $\mathrm{H}$ & -0.47975300 & -3.47206200 & 1.19456500 \\
\hline C & -5.70817100 & 4.01731500 & 0.90547000 \\
\hline $\mathrm{H}$ & -5.72619200 & 3.89200800 & 1.99460100 \\
\hline $\mathrm{H}$ & -6.11276600 & 5.01617200 & 0.68212700 \\
\hline C & 0.54732700 & -2.31805500 & 2.68872200 \\
\hline C & 0.67718200 & -0.94849100 & 2.59861700 \\
\hline C & 1.61709500 & -3.08559100 & 3.23067800 \\
\hline C & 1.85609900 & -0.29019900 & 3.04705200 \\
\hline $\mathrm{H}$ & -0.13221600 & -0.35434700 & 2.18502500 \\
\hline C & 2.77291100 & -2.48099300 & 3.66512200 \\
\hline $\mathrm{H}$ & 1.51054000 & -4.16523900 & 3.30609200 \\
\hline C & 2.00974300 & 1.11994500 & 2.97625700 \\
\hline C & 2.92905100 & -1.06846600 & 3.59238600 \\
\hline
\end{tabular}




\begin{tabular}{lrrr} 
H & 3.57987900 & -3.07809800 & 4.08280100 \\
$\mathrm{C}$ & 3.16032500 & 1.72770700 & 3.42860800 \\
$\mathrm{H}$ & 1.20249000 & 1.71603800 & 2.55761200 \\
$\mathrm{C}$ & 4.09654700 & -0.40851900 & 4.05507400 \\
$\mathrm{C}$ & 4.22279900 & 0.96658300 & 3.98597000 \\
$\mathrm{H}$ & 3.26479400 & 2.80757500 & 3.36781100 \\
$\mathrm{H}$ & 4.90043300 & -1.00663400 & 4.47994300 \\
$\mathrm{C}$ & -4.28465700 & 3.90213100 & 0.38917500 \\
$\mathrm{C}$ & -3.51924300 & 5.02410400 & 0.14860100 \\
$\mathrm{C}$ & -3.72195800 & 2.61230000 & 0.17319400 \\
$\mathrm{C}$ & -2.17471600 & 4.91981300 & -0.30388900 \\
$\mathrm{H}$ & -3.94239200 & 6.01583300 & 0.29882300 \\
$\mathrm{C}$ & -2.43017600 & 2.47630600 & -0.27491900 \\
$\mathrm{H}$ & -4.34518100 & 1.73761400 & 0.32886100 \\
$\mathrm{C}$ & -1.36297800 & 6.05599200 & -0.56521700 \\
$\mathrm{C}$ & -1.61992500 & 3.61784300 & -0.52880000 \\
$\mathrm{H}$ & -2.01098700 & 1.48753400 & -0.44728600 \\
$\mathrm{C}$ & -0.07358700 & 5.91262400 & -1.03202200 \\
$\mathrm{H}$ & -1.77776500 & 7.04770500 & -0.40173000 \\
$\mathrm{C}$ & -0.29154800 & 3.50690200 & -1.00743200 \\
$\mathrm{C}$ & 0.47793600 & 4.62562300 & -1.26105200 \\
$\mathrm{H}$ & 0.52967500 & 6.79403800 & -1.23590100 \\
$\mathrm{H}$ & 0.11653200 & 2.51744900 & -1.19528900 \\
$\mathrm{C}$ & -2.89198100 & -2.65250300 & -2.59831900 \\
$\mathrm{C}$ & -2.35128400 & -3.91836800 & -2.51697400 \\
$\mathrm{C}$ & -2.06353200 & -1.52432000 & -2.33412000 \\
$\mathrm{C}$ & -0.99315400 & -4.12345400 & -2.14818500 \\
$\mathrm{H}$ & -2.97256900 & -4.78958400 & -2.71362300 \\
$\mathrm{C}$ & -0.74855700 & -1.68630500 & -1.96573100 \\
$\mathrm{H}$ & -2.49191900 & -0.52891500 & -2.40426200 \\
$\mathrm{C}$ & -0.42832200 & -5.42036100 & -2.02434700 \\
$\mathrm{C}$ & -0.17660100 & -2.98540000 & -1.84462200 \\
$\mathrm{C}$ & -0.12899800 & -0.81865500 & -1.75187500 \\
$\mathrm{H}$ & 0.86763000 & -5.58487500 & -1.58747700 \\
$\mathrm{H}$ & -1.04142000 & -6.28599900 & -2.26287800 \\
$\mathrm{C}$ & -8.39747400 & -0.30670500 & -1.06733400 \\
$\mathrm{H}$ & -8.77223400 & -0.47382600 & -2.08459400 \\
$\mathrm{H}$ & & & \\
$\mathrm{C}$ & -1.15298800 & -3.19320600 & -1.39388000 \\
$\mathrm{C}$ & 1.67445100 & -4.46663000 & -1.24758800 \\
$\mathrm{H}$ & -2.62452300 & -1.01918600 & 3.47352000 \\
$\mathrm{H}$ & 1.28039000 & -6.58513800 & -1.48114500 \\
$\mathrm{H}$ & 1.75653100 & -2.32586400 & -1.13463900 \\
$\mathrm{H}$ & -4.34878100 & -2.45247100 & -2.93951300 \\
\hline & -4.46728900 & -2.02816200 & -3.95089300 \\
\hline & -4.87201900 & -3.41999900 & -2.92820900 \\
\hline & -8.10964400 & -1.19065300 & -0.23259600 \\
\hline
\end{tabular}

Imaginary Frequency $=0$

$E(R B 3 L Y P)=-4064.5398342$ a.u. 
Conformation \#2 (Pop. = 12.04\%)

Symbolic Z-matrix:

\begin{tabular}{|c|c|c|c|}
\hline & -5.79521100 & -0.67942100 & 3.06253500 \\
\hline - & -6.84753000 & -1.48341600 & 2.31054600 \\
\hline C & -6.39366400 & -1.73257600 & 0.86277600 \\
\hline C & -4.08482100 & -1.72349800 & 1.54199700 \\
\hline C & -4.44988200 & -1.39699700 & 3.00392500 \\
\hline $\mathrm{O}$ & -5.15695700 & -2.41790600 & 0.88694800 \\
\hline Q & -6.27423400 & -0.53391600 & 4.39918600 \\
\hline & -6.31213400 & -0.48570600 & 0.20493300 \\
\hline c & -2.89576500 & -2.67547100 & 1.43504200 \\
\hline $\mathrm{O}$ & -1.77080400 & -2.08148300 & 2.08135500 \\
\hline C & -7.36411200 & 2.00044900 & -1.36809700 \\
\hline C & -8.52265700 & 1.34182300 & -0.62239500 \\
\hline C & -6.84710400 & -0.29604600 & -1.11807000 \\
\hline $\mathrm{O}$ & -6.36942000 & 0.96132700 & -1.54016600 \\
\hline C & -6.38751500 & -1.35919700 & -2.11477800 \\
\hline $\mathrm{O}$ & -4.97855600 & -1.45851900 & -2.10517600 \\
\hline $\mathrm{H}$ & -6.95788900 & -2.46413200 & 2.79441300 \\
\hline$H$ & -8.36327500 & 1.40487100 & 0.46146000 \\
\hline $\mathrm{H}$ & -7.72193800 & 2.30150100 & -2.36283100 \\
\hline $\mathrm{H}$ & -5.69295000 & 0.30619800 & 2.58863200 \\
\hline & -4.54074500 & -2.34325900 & 3.56320500 \\
\hline $\mathrm{H}$ & -3.86107400 & -0.78795700 & 1.01477200 \\
\hline $\mathrm{H}$ & -3.15393900 & -3.62939100 & 1.91959200 \\
\hline $\mathrm{H}$ & -2.67117300 & -2.87570300 & 0.37964800 \\
\hline $\mathrm{H}$ & -6.75884100 & -1.06015900 & -3.10698900 \\
\hline $\mathrm{H}$ & -6.84919000 & -2.32938600 & -1.87133900 \\
\hline C & 4.75469700 & -3.55627000 & -1.41835500 \\
\hline C & 4.94268000 & -2.56876800 & -2.37680100 \\
\hline C & 5.59714300 & -3.57976200 & -0.28580900 \\
\hline C & 5.90420100 & -1.55427600 & -2.24321500 \\
\hline $\mathrm{H}$ & 4.30787000 & -2.60794200 & -3.25742700 \\
\hline C & 6.54383500 & -2.56656500 & -0.13682000 \\
\hline C & 6.70139600 & -1.54043300 & -1.08607000 \\
\hline $\mathrm{H}$ & 7.19668200 & -2.57356900 & 0.72891300 \\
\hline U & 2.71902600 & 1.41178600 & -3.48157900 \\
\hline C & 3.05239300 & 2.64943100 & -2.89676600 \\
\hline C & 3.70866100 & 0.45138800 & -3.63871200 \\
\hline C & 4.36969300 & 2.87208200 & -2.50059000 \\
\hline C & 5.02777100 & 0.64628500 & -3.19662800 \\
\hline $\mathrm{H}$ & 3.42149000 & -0.49013100 & -4.09842500 \\
\hline C & 5.36201300 & 1.88200300 & -2.61530500 \\
\hline & 4.63730800 & 3.83703300 & -2.08159300 \\
\hline 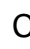 & 6.39319500 & 2.85500400 & 1.69214100 \\
\hline c & 6.72368300 & 1.60289800 & 2.25465900 \\
\hline c & 6.43646300 & 2.99606300 & 0.30552600 \\
\hline 0 & 7.14796700 & 0.58320600 & 1.41041800 \\
\hline 0 & 6.82656000 & 1.94868800 & -0.54796900 \\
\hline & 6.17567000 & 3.95052200 & -0.13720500 \\
\hline 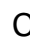 & 7.22293900 & 0.72361600 & 0.01527800 \\
\hline & 6.75274300 & 1.36001700 & 3.60938600 \\
\hline C & 2.04141500 & 3.56185900 & -2.77272700 \\
\hline & 2.05447600 & 4.48697400 & -1.67687600 \\
\hline & 2.74958100 & 4.13118100 & -0.90604500 \\
\hline
\end{tabular}




$\begin{array}{lrrr}\text { H } & 2.41376200 & 5.46339400 & -2.02448000 \\ \mathrm{C} & 6.00427600 & -0.51419900 & -3.35434400 \\ \mathrm{H} & 5.78440000 & -1.01411400 & -4.30455000 \\ \mathrm{H} & 7.02886600 & -0.14706800 & -3.44338300 \\ \mathrm{C} & 6.74409700 & 2.20291400 & -2.05036000 \\ \mathrm{H} & 6.95358600 & 3.26264900 & -2.23420400 \\ \mathrm{H} & 7.51868100 & 1.64744400 & -2.58296800 \\ \mathrm{H} & 7.43520900 & -0.35412300 & 1.87859700 \\ \mathrm{C} & 7.74065100 & -0.46274600 & -0.79641400 \\ \mathrm{H} & 8.55543200 & -0.92413700 & -0.22663400 \\ \mathrm{H} & 8.19335700 & -0.11222100 & -1.72669800 \\ \mathrm{H} & -7.08658900 & -2.40661900 & 0.35739200 \\ \mathrm{H} & -5.63403000 & 0.02590800 & 4.86839700 \\ \mathrm{O} & 3.81122200 & -4.52824700 & -1.65794900 \\ \mathrm{O} & -6.38256400 & 2.93312000 & 0.62455200 \\ \mathrm{O} & -9.74469400 & 1.95868400 & -0.99650500 \\ \mathrm{H} & -10.45059200 & 1.51154000 & -0.50154100 \\ \mathrm{C} & 2.81320400 & -4.76202100 & -0.63788600 \\ \mathrm{H} & 2.93100500 & -5.79031400 & -0.28226800 \\ \mathrm{H} & 2.99307000 & -4.09523200 & 0.20963900 \\ \mathrm{C} & 5.57214100 & 1.64632700 & 4.40376400 \\ \mathrm{H} & 5.42556500 & 2.72607200 & 4.46798300 \\ \mathrm{H} & 5.84495900 & 1.26808900 & 5.39246300 \\ \mathrm{O} & 5.42675400 & -4.62468800 & 0.57842700 \\ \mathrm{O} & 6.06479900 & 3.85783900 & 2.56230800 \\ \mathrm{C} & 1.41990100 & 1.10463200 & -3.82800600 \\ \mathrm{C} & 2.1340 \\ \mathrm{O} & 2.95760500 & -1.03671300 & 3.59266700 \\ \mathrm{C} & 3.52420300 & -3.03606600 & 4.21168500 \\ \mathrm{C} & 3.29388800 & 1.73616400 & 3.27879900 \\ \mathrm{H} & & & \end{array}$




$\begin{array}{lrrr}\text { H } & 1.36085800 & 1.74262000 & 2.35400900 \\ \mathrm{C} & 4.13545700 & -0.39201500 & 4.05074900 \\ \mathrm{C} & 4.31407100 & 0.97105800 & 3.90612900 \\ \mathrm{H} & 3.43885000 & 2.80669900 & 3.16098300 \\ \mathrm{H} & 4.90648000 & -0.99262700 & 4.52960300 \\ \mathrm{C} & -4.07082400 & 3.77665200 & 0.59622400 \\ \mathrm{C} & -3.25210300 & 4.88630100 & 0.55069500 \\ \mathrm{C} & -3.59025700 & 2.53313400 & 0.09862100 \\ \mathrm{C} & -1.93430200 & 4.81180700 & 0.02259000 \\ \mathrm{H} & -3.61194000 & 5.84589900 & 0.91828800 \\ \mathrm{C} & -2.32793900 & 2.43183800 & -0.43687100 \\ \mathrm{H} & -4.25454300 & 1.67517100 & 0.10915500 \\ \mathrm{C} & -1.06806300 & 5.93700500 & -0.03868000 \\ \mathrm{C} & -1.46487900 & 3.56056500 & -0.49415000 \\ \mathrm{H} & -1.97550000 & 1.48171000 & -0.83205000 \\ \mathrm{C} & 0.19067400 & 5.83165500 & -0.58887000 \\ \mathrm{H} & -1.41792000 & 6.89179600 & 0.34650000 \\ \mathrm{C} & -0.16589900 & 3.48719500 & -1.05746500 \\ \mathrm{C} & 0.65557300 & 4.59532700 & -1.10997600 \\ \mathrm{H} & 0.83573500 & 6.70596200 & -0.63562900 \\ \mathrm{H} & 0.17303100 & 2.54496700 & -1.47856300 \\ \mathrm{C} & -3.03554100 & -2.66335100 & -2.78778400 \\ \mathrm{C} & -2.62484000 & -3.90287900 & -2.34532600 \\ \mathrm{C} & -2.07145600 & -1.62482300 & -2.93381500 \\ \mathrm{C} & -1.26959900 & -4.15534700 & -1.99677700 \\ \mathrm{H} & -3.34854800 & -4.70743300 & -2.23264700 \\ \mathrm{C} & -0.75012500 & -1.83354500 & -2.61305500 \\ \mathrm{H} & -2.39490500 & -0.65323100 & -3.29810100 \\ \mathrm{C} & -0.83966700 & -5.41194100 & -1.49640700 \\ \mathrm{C} & -0.31160800 & -3.09533500 & -2.11508600 \\ \mathrm{H} & -0.02433800 & -1.03228400 & -2.73421800 \\ \mathrm{C} & 0.46583100 & -5.60139200 & -1.09918800 \\ \mathrm{H} & -1.56031600 & -6.22236600 & -1.41894500 \\ \mathrm{C} & 1.02476800 & -3.32752400 & -1.69836800 \\ \mathrm{C} & 1.41597800 & -4.54966700 & -1.17963700 \\ \mathrm{H} & 0.77768600 & -6.56634800 & -0.70705400 \\ \mathrm{H} & 1.74176500 & -2.51285600 & -1.77018200 \\ \mathrm{C} & -4.49131500 & -2.39233800 & -3.07229100 \\ \mathrm{H} & -4.63028300 & -1.97842500 & -4.08428800 \\ \mathrm{H} & -5.06489900 & -3.32905500 & -3.01618300 \\ \mathrm{O} & -9.10767600 & -1.04819000 & -0.27776000 \\ \mathrm{H} & -8.90103000 & -0.87859500 & 0.66689100 \\ \mathrm{O} & -3.48864200 & -0.54314200 & 3.61239400 \\ \mathrm{H} & -2.62021200 & -0.95947400 & 3.44850500 \\ \mathrm{C} & -8.10206500 & -0.80792600 & 2.30169700 \\ \mathrm{H} & -8.26248000 & -0.48581100 & 3.20544400 \\ & -8.39055900 & -0.12229200 & -1.06045500 \\ & -8.78435700 & -0.20862900 & -2.08070000\end{array}$

Imaginary Frequency $=0$

$E(R B 3 L Y P)=-4064.5380266$ a.u. 


\section{$P-5 b$}

Conformation \#1 (Pop. = 75.32\%)

Symbolic Z-matrix:

\begin{tabular}{|c|c|c|c|}
\hline C & -5.74916800 & 4.67505000 & -1.18282900 \\
\hline C & -5.74829200 & 4.39628600 & 0.31511100 \\
\hline C & -4.69552300 & 3.33385500 & 0.65252800 \\
\hline C & -3.34115300 & 3.98482800 & -1.22653300 \\
\hline C & -4.34621300 & 5.07931300 & -1.63043600 \\
\hline 0 & -3.42680700 & 3.74490600 & 0.19491800 \\
\hline $\mathrm{O}$ & -6.70400100 & 5.70577700 & -1.41050700 \\
\hline 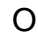 & -7.01343000 & 3.94676800 & 0.78279500 \\
\hline $\mathrm{O}$ & -5.12283000 & 2.11066700 & 0.06935000 \\
\hline & -4.39509700 & 5.24859900 & -3.04762700 \\
\hline & -1.89380300 & 4.32524900 & -1.53925600 \\
\hline 0 & -1.58506900 & 5.60583700 & -1.00634000 \\
\hline n & -5.86001400 & -1.21335500 & 0.51959700 \\
\hline & -5.86225500 & -0.85441600 & 2.02426400 \\
\hline & -4.78213100 & 0.88561900 & 0.72722600 \\
\hline & -5.65849500 & 0.67123400 & 1.99890800 \\
\hline & -5.17436100 & -0.13518000 & -0.16817100 \\
\hline 0 & -6.94016600 & 1.25703500 & 1.85334400 \\
\hline $\mathrm{O}$ & -4.88065700 & -1.54667800 & 2.77432100 \\
\hline & -3.24948800 & 0.83058800 & 0.91966000 \\
\hline & -2.78650900 & -0.45646600 & 1.33694500 \\
\hline & -7.99860900 & -2.44097500 & 0.43561100 \\
\hline & -5.46158800 & 5.31865200 & 0.84010600 \\
\hline & -5.15693600 & 1.01970000 & 2.91270900 \\
\hline & -6.82491600 & -1.08808900 & 2.48377200 \\
\hline & -5.28116900 & -2.12671500 & 0.37028500 \\
\hline & -6.04268600 & 3.76105200 & -1.71990900 \\
\hline & -4.07267900 & 6.01887400 & -1.13722700 \\
\hline & -3.59120900 & 3.06240500 & -1.76683300 \\
\hline & -1.24627800 & 3.55028000 & -1.10806000 \\
\hline & -1.75043200 & 4.32004000 & -2.63052200 \\
\hline & -2.92372400 & 1.55160200 & 1.67436300 \\
\hline & -2.78811700 & 1.10436300 & -0.03181100 \\
\hline & 5.20040600 & 2.55837300 & 3.91397700 \\
\hline & 6.24063200 & 2.43710600 & 3.59389700 \\
\hline & 5.20443600 & 3.22032200 & 4.78472400 \\
\hline & 1.57382400 & -4.04251200 & -3.96615800 \\
\hline & 1.94918100 & -3.02659600 & -4.13548700 \\
\hline & 0.66816200 & -4.19826300 & -4.55408500 \\
\hline & 4.66603300 & 1.32988000 & 4.43296600 \\
\hline & 1.18949000 & -4.22802300 & -2.60537900 \\
\hline & 2.14565300 & -4.08416800 & -1.64076700 \\
\hline & 3.48563100 & -3.79115400 & -1.88601100 \\
\hline & 1.72469000 & -4.24830100 & -0.30392400 \\
\hline & 4.41818400 & -3.62506100 & -0.84619400 \\
\hline & 3.82609400 & -3.67237800 & -2.90818200 \\
\hline & 2.65515300 & -4.12425700 & 0.71947600 \\
\hline & 4.00367000 & -3.80372400 & 0.48464800 \\
\hline & 2.29938900 & -4.27303700 & 1.73542800 \\
\hline & 5.68876300 & 0.28298000 & -2.74794000 \\
\hline & 6.31958600 & 1.06948200 & -1.76699600 \\
\hline
\end{tabular}




\begin{tabular}{|c|c|c|c|}
\hline $\mathrm{C}$ & 5.59050100 & -1.09014300 & -2.54180100 \\
\hline C & 6.74902800 & 0.46138900 & -0.59110400 \\
\hline C & 6.02693400 & -1.71149300 & -1.36237500 \\
\hline $\mathrm{H}$ & 5.12938300 & -1.67885400 & -3.32938600 \\
\hline $\mathrm{C}$ & 6.58431900 & -0.91040700 & -0.34648100 \\
\hline $\mathrm{H}$ & 7.22372800 & 1.09395100 & 0.15381500 \\
\hline C & 4.78200600 & 0.17867800 & 3.69421900 \\
\hline C & 3.85297000 & -0.84421600 & 3.99686500 \\
\hline C & 5.76499600 & -0.07056400 & 2.74443400 \\
\hline C & 3.95544200 & -2.06354200 & 3.33412100 \\
\hline C & 5.86517300 & -1.29898000 & 2.06270500 \\
\hline $\mathrm{H}$ & 6.49539700 & 0.69923800 & 2.52305200 \\
\hline C & 4.94131200 & -2.31140500 & 2.35927300 \\
\hline $\mathrm{O}$ & 2.92271700 & -0.54550000 & 4.95061500 \\
\hline $\mathrm{O}$ & 6.47601900 & 2.43458100 & -1.90476800 \\
\hline $\mathrm{O}$ & 0.39628200 & -4.44351400 & 0.00409500 \\
\hline C & 1.95701900 & -1.54055200 & 5.27433300 \\
\hline $\mathrm{H}$ & 2.42995600 & -2.44148600 & 5.68454800 \\
\hline $\mathrm{H}$ & 1.31213600 & -1.09369100 & 6.03268400 \\
\hline $\mathrm{H}$ & 1.35503000 & -1.81469700 & 4.39916100 \\
\hline C & 7.40557700 & 2.83376400 & -2.92480800 \\
\hline $\mathrm{H}$ & 8.40717600 & 2.44540400 & -2.70383600 \\
\hline $\mathrm{H}$ & 7.08611500 & 2.48456500 & -3.91140100 \\
\hline $\mathrm{H}$ & 7.42576300 & 3.92524000 & -2.90829600 \\
\hline C & 5.83529500 & -3.22187900 & -1.24006000 \\
\hline $\mathrm{H}$ & 6.05878600 & -3.66926300 & -2.21504600 \\
\hline $\mathrm{H}$ & 6.56404500 & -3.64387000 & -0.54515600 \\
\hline C & 6.97983300 & -1.43967400 & 1.02733200 \\
\hline $\mathrm{H}$ & 7.84572300 & -0.86734500 & 1.37893600 \\
\hline $\mathrm{H}$ & 7.31486000 & -2.47708100 & 0.96316300 \\
\hline $\mathrm{H}$ & 3.25606300 & -2.85660500 & 3.57341700 \\
\hline C & 4.93610700 & -3.68197100 & 1.68748700 \\
\hline $\mathrm{H}$ & 4.61603700 & -4.41921100 & 2.43285100 \\
\hline $\mathrm{H}$ & 5.95193400 & -3.97019600 & 1.40705800 \\
\hline $\mathrm{H}$ & 2.33459100 & -4.77120600 & -4.27048600 \\
\hline $\mathrm{H}$ & -4.59283100 & 3.23415300 & 1.73738600 \\
\hline $\mathrm{H}$ & -3.72796400 & 5.90753700 & -3.29497800 \\
\hline $\mathrm{H}$ & -7.69159200 & 4.46424700 & 0.31523900 \\
\hline $\mathrm{H}$ & -6.88107200 & 2.08232600 & 1.32953900 \\
\hline $\mathrm{H}$ & -4.01120200 & -1.31300000 & 2.38527000 \\
\hline $\mathrm{H}$ & -6.67651600 & 5.89772500 & -2.36316200 \\
\hline C & 4.09343500 & 1.75550100 & -3.76259100 \\
\hline $\mathrm{H}$ & 4.42103400 & 2.58432800 & -3.12980500 \\
\hline $\mathrm{H}$ & 3.92297300 & 2.12825700 & -4.77540800 \\
\hline $\mathrm{O}$ & 5.19503900 & 0.81423500 & -3.92149800 \\
\hline C & -0.20147700 & 5.93348900 & -1.05095200 \\
\hline $\mathrm{H}$ & 0.20861300 & 5.75690000 & -2.05665300 \\
\hline $\mathrm{H}$ & -0.16484100 & 7.01418700 & -0.86816100 \\
\hline C & -2.19009500 & -1.26360500 & 0.30089000 \\
\hline $\mathrm{H}$ & -2.91782500 & -1.45354600 & -0.49532800 \\
\hline $\mathrm{H}$ & -1.96640800 & -2.21459400 & 0.79537700 \\
\hline C & 0.63519300 & 5.21014800 & -0.00877100 \\
\hline C & 1.96296400 & 4.91981900 & -0.24518100 \\
\hline C & 0.06515800 & 4.88316700 & 1.25347300 \\
\hline C & 2.78088600 & 4.32424300 & 0.75447400 \\
\hline $\mathrm{H}$ & 2.40969200 & 5.15461800 & -1.20969200 \\
\hline C & 0.82232700 & 4.29058700 & 2.23620900 \\
\hline $\mathrm{H}$ & -0.98721000 & 5.09113000 & 1.41852400 \\
\hline
\end{tabular}




\begin{tabular}{|c|c|c|c|}
\hline & 4.15602400 & 4.03510700 & 0.54469900 \\
\hline & 2.19922000 & 3.99973100 & 2.02423100 \\
\hline & 0.37596600 & 4.03887800 & 3.1953260 \\
\hline & 4.91843900 & 3.46650400 & 1.5428260 \\
\hline & 4.60583400 & 4.26093000 & -0.418766 \\
\hline & 3.01274600 & 3.41041300 & 3.025197 \\
\hline & 4.35262600 & 3.14928200 & 2.805187 \\
\hline & 5.96999400 & 3.25929600 & 1.362295 \\
\hline & 2.56516600 & 3.16419100 & 3.9854700 \\
\hline & -0.92940500 & -0.64887000 & -0.2687 \\
\hline & -0.68571200 & -0.67028000 & -1.627207 \\
\hline & 0.02481600 & -0.05067100 & 0.600974 \\
\hline & 0.49828500 & -0.10564400 & -2.175586 \\
\hline & -1.40882300 & -1.12333100 & -2.302308 \\
\hline & 1.16892900 & 0.52538800 & 0.1018780 \\
\hline & -0.17258300 & -0.03738700 & 1.668876 \\
\hline & 0.77625300 & -0.11816000 & -3.570597 \\
\hline & 1.43646800 & 0.52300100 & -1.295588 \\
\hline & 1.88624100 & 0.99047000 & 0.773056 \\
\hline & 1.91974900 & 0.46683900 & -4.067530 \\
\hline & 0.06529800 & -0.59144700 & -4.243433 \\
\hline & 2.59371700 & 1.13403100 & -1.845192 \\
\hline & 2.84526000 & 1.11563000 & -3.204276 \\
\hline & 2.11585600 & 0.45072200 & -5.136994 \\
\hline & 3.28751000 & 1.62969500 & -1.170588 \\
\hline & -0.17703600 & -5.73382200 & -0.334275 \\
\hline & -0.01384000 & -6.41989900 & 0.505998 \\
\hline & 0.32063700 & -6.13639400 & -1.220396 \\
\hline & -6.57714800 & -4.35227000 & -0.163190 \\
\hline & -5.68705200 & -4.36534700 & -1.217596 \\
\hline & -6.16831900 & -4.87327000 & 1.096523 \\
\hline & -4.34897300 & -4.81305500 & -1.050766 \\
\hline & -5.98812100 & -3.97607200 & -2.188213 \\
\hline & -4.88780800 & -5.33606800 & 1.287702 \\
\hline & -6.87734600 & -4.87844700 & 1.920532 \\
\hline & -3.39011000 & -4.74186400 & -2.098296 \\
\hline & -3.93192100 & -5.28779100 & 0.234684 \\
\hline & -4.58026400 & -5.71794100 & 2.258169 \\
\hline & -2.07432900 & -5.06964300 & -1.869748 \\
\hline & -3.70839600 & -4.39524600 & -3.078549 \\
\hline & -2.56978400 & -5.64211000 & 0.427994 \\
\hline & -1.64492800 & -5.51510600 & -0.588857 \\
\hline & -1.33887200 & -4.97451300 & -2.662614 \\
\hline & -2.25362800 & -5.98490700 & 1.411132 \\
\hline & -7.25227400 & -1.35696800 & -0.113537 \\
\hline & -7.14561200 & -1.46539200 & -1.201758 \\
\hline & -7.82184000 & -0.44498700 & 0.087821 \\
\hline & -7.92538200 & -3.67904500 & -0.286762 \\
\hline & -8.71382600 & -4.29875500 & 0.152563 \\
\hline & -8.17112900 & -3.50874600 & -1.344545 \\
\hline
\end{tabular}

Imaginary Frequency $=0$

$E(R B 3 L Y P)=-4064.5350289 a . u$ 
Conformation \#2 (Pop. = 21.14\%)

Symbolic Z-matrix:

\begin{tabular}{|c|c|c|c|}
\hline & -5.87160200 & 4.53565500 & -1.29345700 \\
\hline C & -5.91307200 & 4.25748200 & 0.20403600 \\
\hline C & -4.84564000 & 3.22310900 & 0.57976200 \\
\hline C & -3.44959500 & 3.90043000 & -1.26335500 \\
\hline C & -4.46490600 & 4.97316900 & -1.69770400 \\
\hline O & -3.57412100 & 3.65814800 & 0.15450100 \\
\hline $\mathrm{O}$ & -6.84275700 & 5.54288000 & -1.55358600 \\
\hline O & -7.18021800 & 3.77535700 & 0.63116900 \\
\hline $\mathrm{O}$ & -5.22776200 & 1.98317300 & 0.00074500 \\
\hline $\mathrm{O}$ & -4.47594200 & 5.14054800 & -3.11598400 \\
\hline C & -1.99855500 & 4.26716300 & -1.52646700 \\
\hline $\mathrm{O}$ & -1.71727900 & 5.53116800 & -0.94083600 \\
\hline C & -5.86437700 & -1.35765200 & 0.53958900 \\
\hline C & -5.87709400 & -0.95475400 & 2.03357500 \\
\hline C & -4.85999000 & 0.77994500 & 0.68369100 \\
\hline C & -5.72550600 & 0.57535400 & 1.96284800 \\
\hline O & -5.23804800 & -0.26811200 & -0.18598500 \\
\hline $\mathrm{O}$ & -7.02601200 & 1.11464800 & 1.80017100 \\
\hline $\mathrm{O}$ & -4.87273500 & -1.59130800 & 2.80236400 \\
\hline & -3.32526000 & 0.76273500 & 0.87049000 \\
\hline $\mathrm{O}$ & -2.82888300 & -0.51075500 & 1.29008900 \\
\hline $\mathrm{O}$ & -7.94158800 & -2.68457700 & 0.52639600 \\
\hline $\mathrm{H}$ & -5.66703100 & 5.18768600 & 0.73603600 \\
\hline & -5.23632500 & 0.96757200 & 2.86542000 \\
\hline $\mathrm{H}$ & -6.83102900 & -1.20660100 & 2.50145900 \\
\hline $\mathrm{H}$ & -5.24236300 & -2.24575200 & 0.41391200 \\
\hline $\mathrm{H}$ & -6.12512000 & 3.61450900 & -1.83892000 \\
\hline $\mathrm{H}$ & -4.22791100 & 5.91893200 & -1.19707000 \\
\hline $\mathrm{H}$ & -3.66417800 & 2.97414200 & -1.81257300 \\
\hline & -1.35547200 & 3.48440800 & -1.10367200 \\
\hline $\mathrm{H}$ & -1.82538600 & 4.29965700 & -2.61286100 \\
\hline $\mathrm{H}$ & -3.01400500 & 1.49639700 & 1.61918200 \\
\hline H & -2.87537500 & 1.04287100 & -0.08448500 \\
\hline c & 4.43786300 & 2.23803800 & 4.60332000 \\
\hline & 4.90263300 & 3.03179400 & 5.19892400 \\
\hline & 3.72155500 & 1.71168700 & 5.23712300 \\
\hline C & 1.45926300 & -3.80385100 & -4.03985000 \\
\hline $\mathrm{H}$ & 1.81335200 & -2.78245500 & -4.22151900 \\
\hline $\mathrm{H}$ & 0.51821700 & -3.96152500 & -4.56876200 \\
\hline C & 5.55274800 & 1.38440500 & 4.28400500 \\
\hline 0 & 1.16712700 & -4.00898700 & -2.65904000 \\
\hline & 2.18356700 & -3.86927400 & -1.75861200 \\
\hline c & 3.50163000 & -3.55986200 & -2.08769000 \\
\hline c & 1.85432600 & -4.06101700 & -0.40017100 \\
\hline c & 4.50171300 & -3.41248900 & -1.11019600 \\
\hline † & 3.77267000 & -3.42058300 & -3.12785500 \\
\hline o & 2.84946900 & -3.94670800 & 0.56190300 \\
\hline U & 4.17837200 & -3.61654500 & 0.24268000 \\
\hline † & 2.56493500 & -4.11954400 & 1.59649900 \\
\hline & 5.62522000 & 0.56290300 & -2.92906300 \\
\hline c & 6.33124700 & 1.30777700 & -1.96657900 \\
\hline & 5.54333100 & -0.81816300 & -2.77833100 \\
\hline & 6.86289600 & 0.64885700 & -0.8624770 \\
\hline
\end{tabular}




\begin{tabular}{|c|c|c|c|}
\hline c & 6.07983500 & -1.49017800 & -1.66968900 \\
\hline $\mathrm{H}$ & 5.01614100 & -1.37228100 & -3.54939100 \\
\hline C & 6.72669400 & -0.73492800 & -0.67291500 \\
\hline $\mathrm{H}$ & 7.39150000 & 1.25023300 & -0.12802900 \\
\hline C & 5.36029200 & 0.22345400 & 3.56635600 \\
\hline C & 4.42367300 & -0.77677100 & 3.90716300 \\
\hline C & 6.24345400 & -0.01950500 & 2.52049000 \\
\hline C & 4.40518700 & -1.95546100 & 3.15887600 \\
\hline C & 6.23968300 & -1.20547300 & 1.77135500 \\
\hline $\mathrm{H}$ & 6.97401500 & 0.75515600 & 2.30510000 \\
\hline C & 5.29233500 & -2.19252500 & 2.09416600 \\
\hline O & 3.61924500 & -0.53518800 & 4.98590000 \\
\hline O & 6.46555700 & 2.67901400 & -2.05162600 \\
\hline O & 0.54999400 & -4.29181500 & -0.01937300 \\
\hline C & 2.64963500 & -1.51759600 & 5.34220700 \\
\hline $\mathrm{H}$ & 3.12600500 & -2.46378100 & 5.62557800 \\
\hline $\mathrm{H}$ & 2.11615300 & -1.10881400 & 6.20139700 \\
\hline $\mathrm{H}$ & 1.94260800 & -1.69641200 & 4.52348100 \\
\hline C & 7.31359200 & 3.13607000 & -3.11701000 \\
\hline $\mathrm{H}$ & 8.33295800 & 2.75259400 & -2.98722900 \\
\hline $\mathrm{H}$ & 6.92671800 & 2.82709500 & -4.09283500 \\
\hline $\mathrm{H}$ & 7.32344400 & 4.22599300 & -3.05236800 \\
\hline C & 5.88904200 & -3.00319400 & -1.59321100 \\
\hline $\mathrm{H}$ & 6.03509500 & -3.41396200 & -2.59846800 \\
\hline $\mathrm{H}$ & 6.66348100 & -3.45615000 & -0.97112100 \\
\hline C & 7.24749400 & -1.32680200 & 0.63185200 \\
\hline $\mathrm{H}$ & 8.15390000 & -0.78234300 & 0.91901800 \\
\hline $\mathrm{H}$ & 7.55397800 & -2.36594600 & 0.49490400 \\
\hline $\mathrm{H}$ & 3.70189300 & -2.73659600 & 3.42476700 \\
\hline C & 5.19551300 & -3.53822400 & 1.37890800 \\
\hline $\mathrm{H}$ & 4.91461700 & -4.29042200 & 2.12500800 \\
\hline $\mathrm{H}$ & 6.18185900 & -3.83900000 & 1.01726200 \\
\hline $\mathrm{H}$ & 2.20488900 & -4.52146500 & -4.40242000 \\
\hline $\mathrm{H}$ & -4.77038600 & 3.13668600 & 1.66801600 \\
\hline $\mathrm{H}$ & -3.82196700 & 5.81864600 & -3.34565300 \\
\hline $\mathrm{H}$ & -7.85658400 & 4.28423500 & 0.15188600 \\
\hline $\mathrm{H}$ & -6.99373000 & 1.91714300 & 1.24038500 \\
\hline $\mathrm{H}$ & -4.01339200 & -1.36282000 & 2.38874900 \\
\hline $\mathrm{H}$ & -6.78624500 & 5.73697300 & -2.50457000 \\
\hline C & 3.94624200 & 2.06236200 & -3.74502500 \\
\hline $\mathrm{H}$ & 4.29530500 & 2.83342500 & -3.05332300 \\
\hline $\mathrm{H}$ & 3.73028400 & 2.52470500 & -4.71131400 \\
\hline $\mathrm{O}$ & 5.04365400 & 1.14882100 & -4.03457400 \\
\hline C & -0.32983300 & 5.82646000 & -0.83362500 \\
\hline $\mathrm{H}$ & 0.17893100 & 5.66821700 & -1.79646200 \\
\hline $\mathrm{H}$ & -0.28945900 & 6.90070100 & -0.61686500 \\
\hline C & -2.20384200 & -1.29747200 & 0.25429600 \\
\hline $\mathrm{H}$ & -2.92180300 & -1.50341600 & -0.54681600 \\
\hline $\mathrm{H}$ & -1.95586400 & -2.24364100 & 0.74589800 \\
\hline C & 0.37863500 & 5.05307300 & 0.26763000 \\
\hline C & 1.73272700 & 4.79337600 & 0.19046400 \\
\hline C & -0.34355500 & 4.64069800 & 1.42052500 \\
\hline C & 2.42342500 & 4.14232700 & 1.24835700 \\
\hline $\mathrm{H}$ & 2.29690400 & 5.09588500 & -0.69013100 \\
\hline C & 0.29063300 & 3.98704600 & 2.45206900 \\
\hline $\mathrm{H}$ & -1.41158200 & 4.82764000 & 1.46009800 \\
\hline C & 3.82302000 & 3.88498900 & 1.20855800 \\
\hline$?$ & 1.68650300 & 3.72401800 & 2.40330300 \\
\hline
\end{tabular}




\begin{tabular}{lrrr} 
H & -0.27258300 & 3.66676800 & 3.32559700 \\
$\mathrm{C}$ & 4.46048600 & 3.26586500 & 2.26014300 \\
$\mathrm{H}$ & 4.39022600 & 4.19320300 & 0.33314600 \\
$\mathrm{C}$ & 2.37176400 & 3.05983000 & 3.45572900 \\
$\mathrm{C}$ & 3.73373500 & 2.84115800 & 3.40677600 \\
$\mathrm{H}$ & 5.53128500 & 3.09129700 & 2.22031900 \\
$\mathrm{H}$ & 1.80034800 & 2.73484800 & 4.32317800 \\
$\mathrm{C}$ & -0.95936500 & -0.64098600 & -0.30303700 \\
$\mathrm{C}$ & -0.73553900 & -0.58797400 & -1.66397100 \\
$\mathrm{C}$ & -0.00216900 & -0.07148900 & 0.58264700 \\
$\mathrm{C}$ & 0.42783500 & 0.02967200 & -2.19921700 \\
$\mathrm{H}$ & -1.46084000 & -1.01792100 & -2.35167700 \\
$\mathrm{C}$ & 1.12446600 & 0.54945300 & 0.09799300 \\
$\mathrm{H}$ & -0.18343700 & -0.11625800 & 1.65264900 \\
$\mathrm{C}$ & 0.68017100 & 0.10065200 & -3.59736700 \\
$\mathrm{C}$ & 1.36861800 & 0.62856600 & -1.30154500 \\
$\mathrm{H}$ & 1.84396900 & 0.99117300 & 0.78208000 \\
$\mathrm{C}$ & 1.80010100 & 0.73970000 & -4.08038500 \\
$\mathrm{H}$ & -0.03296700 & -0.35028300 & -4.28315700 \\
$\mathrm{C}$ & 2.50289200 & 1.29480300 & -1.83598300 \\
$\mathrm{C}$ & 2.72717000 & 1.36038200 & -3.19808300 \\
$\mathrm{H}$ & 1.97634800 & 0.78888300 & -5.15227700 \\
$\mathrm{H}$ & 3.19958400 & 1.76594500 & -1.14660700 \\
$\mathrm{C}$ & 0.02232400 & -5.61824400 & -0.28748600 \\
$\mathrm{H}$ & 0.23867200 & -6.26096100 & 0.57475000 \\
$\mathrm{H}$ & 0.51520800 & -6.03655000 & -1.16962800 \\
$\mathrm{C}$ & -6.43498300 & -4.54588100 & -0.02185500 \\
$\mathrm{C}$ & -5.56806900 & -4.56371100 & -1.09521000 \\
$\mathrm{C}$ & -5.97345500 & -4.98756000 & 1.24996900 \\
$\mathrm{C}$ & -4.20593200 & -4.93644100 & -0.93992200 \\
$\mathrm{H}$ & -5.90900900 & -4.23575000 & -2.07525800 \\
$\mathrm{C}$ & -4.66720200 & -5.37645400 & 1.43168900 \\
$\mathrm{H}$ & -6.66354000 & -4.98999100 & 2.08987300 \\
$\mathrm{C}$ & -3.27575400 & -4.86925400 & -2.01295700 \\
$\mathrm{C}$ & -3.73724100 & -5.32840400 & 0.35561700 \\
$\mathrm{H}$ & -4.32012700 & -5.69709300 & 2.41094100 \\
$\mathrm{C}$ & -1.94057300 & -5.12050100 & -1.80160000 \\
$\mathrm{C}$ & -3.63307000 & -4.58759100 & -3.00054300 \\
$\mathrm{H}$ & -8.56549000 & -4.58613100 & 0.33629900 \\
$\mathrm{C}$ & -8.09167800 & -3.83523600 & -1.20363800 \\
$\mathrm{C}$ & -2.35455900 & -5.60391600 & 0.53231200 \\
$\mathrm{C}$ & -1.46012600 & -5.47972200 & -0.51199100 \\
$\mathrm{H}$ & -1.22903400 & -5.03028300 & -2.61631600 \\
& -1.99993400 & -5.88382700 & 1.52224200 \\
\hline
\end{tabular}

Imaginary Frequency $=0$

$E(R B 3 L Y P)=-4064.5338306 \mathrm{a} . \mathrm{u}$ 


\section{$M-5 b$}

Conformation \#1 (Pop. = 52.95\%)

Symbolic Z-matrix:

\begin{tabular}{|c|c|c|c|}
\hline & 7.28986400 & -2.78527500 & -2 \\
\hline & 6.36770200 & -1.69230000 & -2.72 \\
\hline & 6.46688500 & -0.44477000 & -1.84 \\
\hline & 6.92881700 & -3.07787100 & -0.6918 \\
\hline & 6.06553100 & -0.75992500 & -0.51859 \\
\hline & 8.62321500 & -2.30392400 & -2.3196 \\
\hline & 5.03220200 & -2.20045200 & \\
\hline & 5.58923900 & 0.51314600 & $-2.405 \varsigma$ \\
\hline & 5.72002400 & -3.82818900 & -0.633 \\
\hline & 5.55318600 & 2.35399000 & -0.6954 \\
\hline & 4.15557800 & 2.22111700 & -2.61994 \\
\hline & 6.85089900 & 1.96081700 & -0.302458 \\
\hline & 5.86480400 & 4.81885700 & $-0.840 \varepsilon$ \\
\hline & 6.68391800 & -1.42963400 & -3.745 \\
\hline & 4.92399600 & 2.59234500 & 0.170891 \\
\hline & 7.13452600 & -3.70927400 & -2.73981 \\
\hline & 7.70891100 & -3.71360000 & -0.2540 \\
\hline & -0.86786500 & -3.53945600 & 2.167253 \\
\hline & -6.36437400 & 2.74559900 & 1.016938 \\
\hline & -7.01704100 & 1.53558600 & 0.81524 \\
\hline & -6.81396300 & 0.41539000 & 1.63837 \\
\hline & -7.72599400 & 1.47898400 & -0.0070000 \\
\hline & -5.27151800 & 1.77029400 & 2.931412 \\
\hline & -5.90615200 & 0.53565000 & 2.706099 \\
\hline & -4.60052300 & 1.85639300 & 3.778681 \\
\hline & -6.87843000 & -2.28916600 & $-2.11979 \varepsilon$ \\
\hline & -5.97436700 & -3.33504100 & -1.83057 \\
\hline & -7.36709700 & -1.51532200 & -1.066643 \\
\hline & -5.64313300 & -3.57876100 & -0.501578 \\
\hline & -7.01735100 & -1.75638500 & 0.273234 \\
\hline & -8.06682100 & -0.71514300 & -1.279850 \\
\hline & -6.15056300 & -2.82270700 & 0.56625 \\
\hline & -4.97561700 & -4.41399700 & -0.3085020 \\
\hline & -1.99011900 & -2.81357200 & 2.507497 \\
\hline & -1.90507900 & -1.65428700 & 3.298844 \\
\hline & -3.24381600 & -3.27385300 & 2.120459 \\
\hline & -3.07650900 & -0.98401000 & 3.641510 \\
\hline & -4.42991000 & -2.60720200 & 2.463045 \\
\hline & -3.28284000 & -4.18916900 & 1.5362310 \\
\hline & -4.34416400 & -1.42714000 & 3.229077 \\
\hline & -7.62012100 & -0.84604400 & 1.338083 \\
\hline & -8.61046300 & -0.53148400 & 0.989766 \\
\hline & -7.79762600 & -1.40749200 & 2.258382 \\
\hline & -5.74308700 & -3.21606800 & $1.982503 c$ \\
\hline & -5.63228700 & -4.30588000 & 2.007245 \\
\hline & -6.54771400 & -2.98589600 & 2.684241 \\
\hline & -2.98488200 & -0.09527900 & 4.259993 \\
\hline & -5.56163600 & -0.60944700 & 3.655702 \\
\hline & -5.35002800 & -0.17974800 & 4.64089 \\
\hline & -6.42639100 & -1.26172300 & 3.79642 \\
\hline & & & \\
\hline
\end{tabular}




\begin{tabular}{|c|c|c|c|}
\hline $\mathrm{H}$ & 5.11569300 & -3.37682900 & -1.25974100 \\
\hline $\mathrm{H}$ & 9.23012000 & -3.02807700 & -2.09723500 \\
\hline $\mathrm{H}$ & 4.41686100 & -1.46871300 & -2.48804600 \\
\hline $\mathrm{H}$ & 6.82266900 & 1.17604400 & 0.27929200 \\
\hline & 6.69449100 & 4.73653100 & -0.34265200 \\
\hline & -0.06427200 & -2.94621100 & 1.13461100 \\
\hline & -0.63473900 & -2.86164400 & 0.20186300 \\
\hline & 0.30087400 & -1.95975500 & 1.43596100 \\
\hline & 0.78231700 & -3.61908000 & 0.98340600 \\
\hline & -7.23344900 & -2.11931400 & -3.43032000 \\
\hline & -8.17755200 & -1.09910300 & -3.75029900 \\
\hline $\mathrm{H}$ & -7.80097800 & -0.10573800 & -3.47936600 \\
\hline & -8.31584200 & -1.15294600 & -4.83091100 \\
\hline & -9.13679000 & -1.27473000 & -3.24925800 \\
\hline 0 & -0.68805000 & -1.14789600 & 3.70366700 \\
\hline 0 & -5.49068800 & -4.21857400 & -2.76796300 \\
\hline & -5.48441200 & 2.87966700 & 2.11061800 \\
\hline $\mathrm{O}$ & -4.92020300 & 4.10540200 & 2.29516800 \\
\hline$C$ & -3.99653100 & 4.26463600 & 3.36763000 \\
\hline $\mathrm{H}$ & -3.13672900 & 3.59253500 & 3.25844800 \\
\hline & -3.65653800 & 5.29997500 & 3.31457300 \\
\hline & -4.47394200 & 4.08802700 & 4.33934600 \\
\hline C & 5.70227700 & 3.61064500 & -1.55921100 \\
\hline H & 6.56437900 & 3.43565100 & -2.21993000 \\
\hline C & 6.81763300 & -1.79152100 & 0.15665100 \\
\hline $\mathrm{H}$ & 6.18940400 & -2.04106200 & 1.01389800 \\
\hline C & 8.15509700 & -1.24509600 & 0.67581400 \\
\hline-1 & 8.82266000 & -0.99579700 & -0.15243900 \\
\hline H & 8.64627300 & -2.01902500 & 1.28211600 \\
\hline 0 & 8.00329100 & -0.04481800 & 1.43559800 \\
\hline C & 4.76192600 & 1.38627500 & -1.63371500 \\
\hline C & 3.62599000 & 0.60581100 & -0.93941900 \\
\hline $\mathrm{H}$ & 4.00357600 & 0.08864300 & -0.05971000 \\
\hline $\mathrm{H}$ & 2.86746700 & 1.33456800 & -0.63765200 \\
\hline C & 4.43280400 & 3.61790400 & -2.41244400 \\
\hline $\mathrm{H}$ & 4.61790100 & 4.05651100 & -3.39949100 \\
\hline 0 & 3.04742500 & -0.40478600 & -1.76517900 \\
\hline C & 3.23250100 & 4.35520900 & -1.82534300 \\
\hline $\mathrm{H}$ & 2.37213200 & 4.21603300 & -2.49626100 \\
\hline $\mathrm{H}$ & 3.46798100 & 5.42907300 & -1.79108400 \\
\hline $\mathrm{O}$ & 2.93989800 & 3.87392800 & -0.52125300 \\
\hline $\mathrm{O}$ & -6.63113200 & 3.83095400 & 0.20523600 \\
\hline C & -5.97428900 & 3.81580300 & -1.08926300 \\
\hline $\mathrm{H}$ & -6.10154300 & 2.82819300 & -1.54759900 \\
\hline $\mathrm{H}$ & -6.52711500 & 4.54981900 & -1.68144200 \\
\hline C & -4.85218600 & -3.72609900 & -3.97542100 \\
\hline $\mathrm{H}$ & -5.58795800 & -3.20484900 & -4.59038300 \\
\hline $\mathrm{H}$ & -4.55074200 & -4.64422000 & -4.48523000 \\
\hline C & 0.05321900 & -1.96349900 & 4.63157500 \\
\hline $\mathrm{H}$ & -0.23709700 & -1.67169000 & 5.65181400 \\
\hline $\mathrm{H}$ & -0.20823300 & -3.01657900 & 4.49866700 \\
\hline C & 7.72611200 & -0.19731400 & 2.83871200 \\
\hline $\mathrm{H}$ & 8.14238700 & 0.70525100 & 3.29981000 \\
\hline $\mathrm{H}$ & 8.27559900 & -1.06224700 & 3.23153300 \\
\hline C & 2.17152000 & 0.05833100 & -2.81229600 \\
\hline $\mathrm{H}$ & 1.83833600 & 1.07602800 & -2.59465700 \\
\hline $\mathrm{H}$ & 2.74121400 & 0.09952300 & -3.75019100 \\
\hline 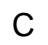 & 1.94494400 & 4.61965400 & 0.17636900 \\
\hline
\end{tabular}




\begin{tabular}{|c|c|c|c|}
\hline & 2.10910700 & 4.38135700 & 1.23417300 \\
\hline $\mathrm{H}$ & 2.11638700 & 5.69812600 & 0.04916200 \\
\hline C & 6.25224300 & -0.31205600 & 3.16501000 \\
\hline C & 5.73069100 & -1.43675300 & 3.77062400 \\
\hline C & 5.37944400 & 0.76335800 & 2.83604300 \\
\hline C & 4.33882200 & -1.55805700 & 4.03581400 \\
\hline $\mathrm{H}$ & 6.38557500 & -2.26308500 & 4.04037400 \\
\hline C & 4.02731000 & 0.67663300 & 3.07149200 \\
\hline $\mathrm{H}$ & 5.79607900 & 1.66150900 & 2.38715800 \\
\hline C & 3.77645800 & -2.71748900 & 4.63405500 \\
\hline C & 3.46249200 & -0.48988400 & 3.65949500 \\
\hline $\mathrm{H}$ & 3.37032200 & 1.50170100 & 2.80726500 \\
\hline C & 2.41707900 & -2.82532100 & 4.81919400 \\
\hline $\mathrm{H}$ & 4.43492700 & -3.53208000 & 4.92536500 \\
\hline C & 2.06490500 & -0.63084700 & 3.86931100 \\
\hline C & 1.53857600 & -1.78001800 & 4.42354000 \\
\hline $\mathrm{H}$ & 2.00264500 & -3.72919800 & 5.25937500 \\
\hline $\mathrm{H}$ & 1.40335000 & 0.17328800 & 3.56122800 \\
\hline C & 0.97962300 & -0.85913200 & -2.94653500 \\
\hline C & -0.28207100 & -0.32225400 & -3.09811700 \\
\hline C & 1.13847300 & -2.27355400 & -2.97144900 \\
\hline C & -1.42297100 & -1.14551600 & -3.29474200 \\
\hline $\mathrm{H}$ & -0.41976200 & 0.75655500 & -3.07392900 \\
\hline C & 0.05230700 & -3.10096300 & -3.14324000 \\
\hline $\mathrm{H}$ & 2.13117200 & -2.69842400 & -2.85307200 \\
\hline C & -2.72283400 & -0.60529300 & -3.48575800 \\
\hline C & -1.25686800 & -2.56812500 & -3.31597800 \\
\hline $\mathrm{H}$ & 0.18462500 & -4.18009900 & -3.16080700 \\
\hline C & -3.80301500 & -1.43071300 & -3.69987400 \\
\hline $\mathrm{H}$ & -2.85005500 & 0.47452200 & -3.47348600 \\
\hline C & -2.39582900 & -3.38921000 & -3.52816600 \\
\hline C & -3.65123500 & -2.84448600 & -3.72512100 \\
\hline $\mathrm{H}$ & -4.78867000 & -1.00599500 & -3.86724200 \\
\hline $\mathrm{H}$ & -2.26327500 & -4.46922000 & -3.55164600 \\
\hline C & 0.51976300 & 4.26558700 & -0.20250100 \\
\hline C & -0.43438500 & 5.24474900 & -0.38976500 \\
\hline C & 0.13240200 & 2.89894600 & -0.28384900 \\
\hline C & -1.79839900 & 4.91672100 & -0.62374100 \\
\hline $\mathrm{H}$ & -0.15454800 & 6.29504400 & -0.33321800 \\
\hline C & -1.17203700 & 2.54549500 & -0.53669300 \\
\hline $\mathrm{H}$ & 0.88015000 & 2.12795800 & -0.12787300 \\
\hline C & -2.80898100 & 5.90773100 & -0.76309300 \\
\hline C & -2.17711900 & 3.53789900 & -0.70069800 \\
\hline $\mathrm{H}$ & -1.45528300 & 1.49729500 & -0.59072000 \\
\hline C & -4.12675100 & 5.55004100 & -0.93237300 \\
\hline $\mathrm{H}$ & -2.52575400 & 6.95662800 & -0.71868200 \\
\hline C & -3.54265100 & 3.20561000 & -0.90326900 \\
\hline C & -4.51361500 & 4.18296400 & -0.99585300 \\
\hline $\mathrm{H}$ & -4.89078000 & 6.31919100 & -1.01651300 \\
\hline 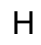 & -3.81989200 & 2.15521300 & -0.96051200 \\
\hline
\end{tabular}

Imaginary Frequency $=0$

$E(R B 3 L Y P)=-4064.5224152$ a.u. 
Conformation \#2 (Pop. = 40.31\%)

Symbolic Z-matrix:

\begin{tabular}{|c|c|c|c|}
\hline C & -7.28565600 & 2.80017600 & -2.15052900 \\
\hline C & -6.36295200 & 1.70456900 & -2.71924400 \\
\hline C & -6.46544300 & 0.45336100 & -1.84150400 \\
\hline C & -6.92819300 & 3.07877600 & -0.67757700 \\
\hline $\mathrm{O}$ & -6.06490800 & 0.76104600 & -0.51519000 \\
\hline $\mathrm{O}$ & -8.65158600 & 2.39592500 & -2.20225600 \\
\hline $\mathrm{O}$ & -5.02551800 & 2.20834000 & -2.72495100 \\
\hline $\mathrm{O}$ & -5.59203100 & -0.50631400 & -2.40734700 \\
\hline $\mathrm{O}$ & -5.71554100 & 3.82174600 & -0.61373400 \\
\hline C & -5.55854100 & -2.35126200 & -0.70111500 \\
\hline $\mathrm{O}$ & -4.16197800 & -2.21654300 & -2.62681200 \\
\hline $\mathrm{O}$ & -6.85744300 & -1.95893300 & -0.31142500 \\
\hline $\mathrm{O}$ & -5.86723300 & -4.81644500 & -0.84699800 \\
\hline $\mathrm{H}$ & -6.67492600 & 1.44175800 & -3.74133000 \\
\hline $\mathrm{H}$ & -4.93129200 & -2.58935700 & 0.16667200 \\
\hline $\mathrm{H}$ & -7.12738100 & 3.73050000 & -2.71209300 \\
\hline $\mathrm{H}$ & -7.71054500 & 3.71239500 & -0.24485800 \\
\hline $\mathrm{O}$ & 0.87072900 & 3.54757700 & 2.15640700 \\
\hline C & 6.36112800 & -2.74627600 & 1.02811400 \\
\hline C & 7.01506100 & -1.53756200 & 0.82274300 \\
\hline C & 6.81284100 & -0.41453500 & 1.64221600 \\
\hline $\mathrm{H}$ & 7.72436100 & -1.48428800 & 0.00057200 \\
\hline C & 5.26884100 & -1.76384600 & 2.93925200 \\
\hline C & 5.90474100 & -0.53054300 & 2.71016800 \\
\hline $\mathrm{H}$ & 4.59762700 & -1.84663900 & 3.78667400 \\
\hline C & 6.88060100 & 2.27866000 & -2.12429200 \\
\hline C & 5.97779000 & 3.32651100 & -1.83834600 \\
\hline C & 7.36815100 & 1.50737400 & -1.06873900 \\
\hline C & 5.64662400 & 3.57458600 & -0.51014500 \\
\hline C & 7.01852300 & 1.75289800 & 0.27036000 \\
\hline $\mathrm{H}$ & 8.06696200 & 0.70573200 & -1.27944900 \\
\hline C & 6.15296600 & 2.82113200 & 0.56003600 \\
\hline $\mathrm{H}$ & 4.98009600 & 4.41120900 & -0.31967700 \\
\hline C & 1.99208400 & 2.82163900 & 2.49948300 \\
\hline C & 1.90567000 & 1.66465100 & 3.29404400 \\
\hline C & 3.24639600 & 3.27976600 & 2.11186300 \\
\hline C & 3.07637800 & 0.99451000 & 3.63941200 \\
\hline C & 4.43174900 & 2.61311300 & 2.45697700 \\
\hline $\mathrm{H}$ & 3.28648500 & 4.19332900 & 1.52497300 \\
\hline C & 4.34462600 & 1.43541000 & 3.22646000 \\
\hline C & 7.62024700 & 0.84518100 & 1.33806100 \\
\hline $\mathrm{H}$ & 8.61030300 & 0.52857100 & 0.99079900 \\
\hline $\mathrm{H}$ & 7.79821900 & 1.40928400 & 2.25663900 \\
\hline C & 5.74566400 & 3.21923400 & 1.97500400 \\
\hline $\mathrm{H}$ & 5.63597800 & 4.30922700 & 1.99642100 \\
\hline $\mathrm{H}$ & 6.54989400 & 2.99034400 & 2.67761600 \\
\hline $\mathrm{H}$ & 2.98362300 & 0.10760200 & 4.26033500 \\
\hline C & 5.56117000 & 0.61791300 & 3.65604900 \\
\hline $\mathrm{H}$ & 5.34889100 & 0.19157300 & 4.64255300 \\
\hline $\mathrm{H}$ & 6.42653700 & 1.26977600 & 3.79489600 \\
\hline $\mathrm{H}$ & -7.48669200 & 0.05896000 & -1.85165800 \\
\hline $\mathrm{H}$ & -5.11022700 & 3.37023100 & -1.23903300 \\
\hline
\end{tabular}




\begin{tabular}{|c|c|c|c|}
\hline H & -8.90217400 & 2.29178900 & -3.13480000 \\
\hline $\mathrm{H}$ & -4.41137100 & 1.47360900 & -2.48877600 \\
\hline $\mathrm{H}$ & -6.83089800 & -1.17961800 & 0.27781700 \\
\hline $\mathrm{H}$ & -6.69637700 & -4.73422400 & -0.34788500 \\
\hline C & 0.06664100 & 2.95176100 & 1.12561700 \\
\hline $\mathrm{H}$ & 0.63730800 & 2.86306300 & 0.19337800 \\
\hline $\mathrm{H}$ & -0.29998800 & 1.96690300 & 1.43036800 \\
\hline $\mathrm{H}$ & -0.77893100 & 3.62529400 & 0.97171700 \\
\hline 0 & 7.23568600 & 2.10449000 & -3.43423800 \\
\hline C & 8.17874800 & 1.08230900 & -3.75098100 \\
\hline $\mathrm{H}$ & 7.80102700 & 0.09015300 & -3.47723400 \\
\hline $\mathrm{H}$ & 8.31736400 & 1.13282500 & -4.83171200 \\
\hline $\mathrm{H}$ & 9.13805500 & 1.25835700 & -3.25021900 \\
\hline 0 & 0.68808100 & 1.15984500 & 3.69916600 \\
\hline 0 & 5.49541600 & 4.20794500 & -2.77838700 \\
\hline C & 5.48072900 & -2.87598900 & 2.12195300 \\
\hline O & 4.91515500 & -4.10055200 & 2.31015900 \\
\hline C & 3.99152800 & -4.25556800 & 3.38328600 \\
\hline $\mathrm{H}$ & 3.13236200 & -3.58296300 & 3.27220900 \\
\hline $\mathrm{H}$ & 3.65050800 & -5.29073200 & 3.33350600 \\
\hline $\mathrm{H}$ & 4.46930500 & -4.07642100 & 4.35435700 \\
\hline C & -5.70555000 & -3.60802900 & -1.56516000 \\
\hline $\mathrm{H}$ & -6.56756700 & -3.43377300 & -2.22622800 \\
\hline C & -6.81938500 & 1.78795200 & 0.16430600 \\
\hline $\mathrm{H}$ & -6.19280200 & 2.03469000 & 1.02351700 \\
\hline C & -8.15636000 & 1.23768800 & 0.68050400 \\
\hline $\mathrm{H}$ & -8.82341200 & 0.98713800 & -0.14744600 \\
\hline $\mathrm{H}$ & -8.64982400 & 2.00909100 & 1.28792700 \\
\hline $\mathrm{O}$ & -8.00071900 & 0.03628000 & 1.43858200 \\
\hline C & -4.76579300 & -1.38279900 & -1.63780400 \\
\hline C & -3.62764800 & -0.60603300 & -0.94303500 \\
\hline $\mathrm{H}$ & -4.00360300 & -0.08843400 & -0.06290600 \\
\hline $\mathrm{H}$ & -2.87104600 & -1.33702800 & -0.64204400 \\
\hline C & -4.43599000 & -3.61375300 & -2.41807500 \\
\hline $\mathrm{H}$ & -4.62003700 & -4.05324100 & -3.40492300 \\
\hline 0 & -3.04646100 & 0.40360000 & -1.76835600 \\
\hline C & -3.23472600 & -4.34910900 & -1.83031600 \\
\hline $\mathrm{H}$ & -2.37409900 & -4.20844500 & -2.50058800 \\
\hline $\mathrm{H}$ & -3.46865600 & -5.42332500 & -1.79658700 \\
\hline O & -2.94379900 & -3.86795400 & -0.52579900 \\
\hline O & 6.62707200 & -3.83457000 & 0.22010200 \\
\hline C & 5.97198100 & -3.82238100 & -1.07528300 \\
\hline $\mathrm{H}$ & 6.10098700 & -2.83624200 & -1.53628100 \\
\hline $\mathrm{H}$ & 6.52476000 & -4.55870800 & -1.66463000 \\
\hline C & 4.85679100 & 3.71287100 & -3.98469300 \\
\hline $\mathrm{H}$ & 5.59219700 & 3.18918500 & -4.59801800 \\
\hline $\mathrm{H}$ & 4.55647100 & 4.62992800 & -4.49707600 \\
\hline C & -0.05377200 & 1.97811100 & 4.62402900 \\
\hline $\mathrm{H}$ & 0.23935600 & 1.69314500 & 5.64542000 \\
\hline $\mathrm{H}$ & 0.20393600 & 3.03130400 & 4.48460700 \\
\hline C & -7.72317600 & 0.18706600 & 2.84176500 \\
\hline 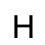 & -8.13636400 & -0.71760600 & 3.30153800 \\
\hline $\mathrm{H}$ & -8.27513700 & 1.04958700 & 3.23639500 \\
\hline C & -2.17142700 & -0.06113000 & -2.81549200 \\
\hline $\mathrm{H}$ & -1.83933900 & -1.07906100 & -2.59725900 \\
\hline П & -2.74135900 & -0.10229200 & -3.75324200 \\
\hline C & -1.95128800 & -4.61472700 & 0.17420800 \\
\hline & -2.11744000 & -4.37577800 & 1.23155600 \\
\hline
\end{tabular}




\begin{tabular}{lrrr} 
H & -2.12398200 & -5.69300100 & 0.04705700 \\
$\mathrm{C}$ & -6.24944300 & 0.30590400 & 3.16729800 \\
$\mathrm{C}$ & -5.73094000 & 1.43124400 & 3.77438200 \\
$\mathrm{C}$ & -5.37341400 & -0.76585600 & 2.83516900 \\
$\mathrm{C}$ & -4.33909700 & 1.55697700 & 4.03756000 \\
$\mathrm{H}$ & -6.38828400 & 2.25486900 & 4.04640900 \\
$\mathrm{C}$ & -4.02123900 & -0.67486500 & 3.06877800 \\
$\mathrm{H}$ & -5.78755300 & -1.66468500 & 2.38532400 \\
$\mathrm{C}$ & -3.77976900 & 2.71748300 & 4.63665800 \\
$\mathrm{C}$ & -3.45962100 & 0.49264500 & 3.65781700 \\
$\mathrm{H}$ & -3.36174900 & -1.49712600 & 2.80203600 \\
$\mathrm{C}$ & -2.42044000 & 2.83007900 & 4.81910000 \\
$\mathrm{H}$ & -4.44065500 & 3.52915000 & 4.93063100 \\
$\mathrm{C}$ & -2.06210200 & 0.63843500 & 3.86494600 \\
$\mathrm{C}$ & -1.53889500 & 1.78870400 & 4.41973700 \\
$\mathrm{H}$ & -2.00842200 & 3.73480800 & 5.25982000 \\
$\mathrm{H}$ & -1.39820100 & -0.16266500 & 3.55410400 \\
$\mathrm{C}$ & -0.97848100 & 0.85480200 & -2.95072700 \\
$\mathrm{C}$ & 0.28264700 & 0.31618100 & -3.10084300 \\
$\mathrm{C}$ & -1.13568700 & 2.26936600 & -2.97840900 \\
$\mathrm{C}$ & 1.42455800 & 1.13772200 & -3.29877100 \\
$\mathrm{H}$ & 0.41908300 & -0.76273400 & -3.07450000 \\
$\mathrm{C}$ & -0.04851000 & 3.09516300 & -3.15158400 \\
$\mathrm{H}$ & -2.12790000 & 2.69567200 & -2.86106100 \\
$\mathrm{C}$ & 2.72383500 & 0.59559500 & -3.48834200 \\
$\mathrm{C}$ & 1.26008900 & 2.56046900 & -3.32299700 \\
$\mathrm{H}$ & -0.17957300 & 4.17441400 & -3.17131000 \\
$\mathrm{C}$ & 3.80499700 & 1.41931500 & -3.70404700 \\
$\mathrm{H}$ & 2.84981800 & -0.48433500 & -3.47367200 \\
$\mathrm{C}$ & 2.40001800 & 3.37979100 & -3.53680300 \\
$\mathrm{C}$ & 3.65481900 & 2.83319500 & -3.73244100 \\
$\mathrm{H}$ & 4.79020900 & 0.99312200 & -3.87026500 \\
$\mathrm{H}$ & 2.26871800 & 4.45989900 & -3.56263400 \\
$\mathrm{C}$ & -0.52472300 & -4.26287200 & -0.20159800 \\
$\mathrm{C}$ & 0.42882100 & -5.24351300 & -0.38423100 \\
$\mathrm{C}$ & -0.13550700 & -2.89686000 & -0.28455700 \\
$\mathrm{C}$ & 1.79383100 & -4.91754300 & -0.61527100 \\
$\mathrm{H}$ & 0.14762600 & -6.29336800 & -0.32627600 \\
$\mathrm{C}$ & 1.17000600 & -2.54544500 & -0.53471900 \\
$\mathrm{H}$ & -0.88269600 & -2.12464600 & -0.13208500 \\
$\mathrm{C}$ & 2.80363500 & -5.90998600 & -0.75001600 \\
$\mathrm{C}$ & 2.17433800 & -3.53932700 & -0.69421100 \\
$\mathrm{H}$ & 1.45459700 & -1.49768400 & -0.59011500 \\
$\mathrm{H}$ & 4.12218200 & -5.55415100 & -0.91718400 \\
$\mathrm{C}$ & 2.51914300 & -6.95846600 & -0.70389400 \\
$\mathrm{H}$ & 3.54068600 & -3.20901700 & -0.89453900 \\
$\mathrm{C}$ & 4.51074000 & -4.18765700 & -0.98305000 \\
& 4.88551500 & -6.32435300 & -0.99795200 \\
& 3.81920000 & -2.15905900 & -0.95362000 \\
\hline
\end{tabular}

Imaginary Frequency $=0$

$E(R B 3 L Y P)=-4064.5221581$ a.u 


\section{M-5aсAch}

Symbolic Z-matrix:

$\begin{array}{rrrr}\mathrm{C} & -5.498030 & -0.383334 & 2.720378 \\ \mathrm{C} & -6.721538 & -1.171521 & 2.276302 \\ \mathrm{C} & -6.476420 & -1.690676 & 0.852081 \\ \mathrm{C} & -4.139629 & -2.006684 & 1.314958 \\ \mathrm{C} & -4.270402 & -1.284733 & 2.677047 \\ \mathrm{O} & -5.380700 & -2.580580 & 0.882503 \\ \mathrm{O} & -5.767596 & 0.094652 & 4.040015 \\ \mathrm{O} & -6.246423 & -0.562649 & 0.035516 \\ \mathrm{C} & -3.186685 & -3.197706 & 1.423700 \\ \mathrm{O} & -1.934671 & -2.737743 & 1.925576 \\ \mathrm{C} & -7.119786 & 1.896969 & -1.579752 \\ \mathrm{C} & -8.307092 & 1.411306 & -0.752283 \\ \mathrm{C} & -6.860678 & -0.428615 & -1.251027 \\ \mathrm{O} & -6.305332 & 0.728763 & -1.834351 \\ \mathrm{C} & -6.564971 & -1.619343 & -2.160904 \\ \mathrm{O} & -5.171807 & -1.828898 & -2.225398 \\ \mathrm{H} & -6.857885 & -2.039497 & 2.936833 \\ \mathrm{H} & -8.088601 & 1.512242 & 0.319038 \\ \mathrm{H} & -7.500504 & 2.266142 & -2.542050 \\ \mathrm{H} & -5.354235 & 0.458284 & 2.031573 \\ \mathrm{H} & -4.389310 & -2.044908 & 3.467639 \\ \mathrm{H} & -3.784296 & -1.288918 & 0.566108 \\ \mathrm{H} & -3.631494 & -3.929006 & 2.115449 \\ \mathrm{H} & -3.059476 & -3.678850 & 0.445799 \\ \mathrm{H} & -6.982059 & -1.397765 & -3.156605 \\ \mathrm{H} & -7.080087 & -2.515630 & -1.777361 \\ \mathrm{C} & 4.613090 & -3.415787 & -1.837405 \\ \mathrm{C} & 4.839327 & -2.307300 & -2.648422 \\ \mathrm{C} & 5.419758 & -3.585065 & -0.687776 \\ \mathrm{C} & 5.775671 & -1.309357 & -2.335551 \\ \mathrm{H} & 4.258634 & -2.243205 & -3.565113 \\ \mathrm{C} & 6.314541 & -2.572099 & -0.345553 \\ \mathrm{C} & 6.493100 & -1.423063 & -1.134346 \\ \mathrm{H} & 6.909184 & -2.673439 & 0.555386 \\ \mathrm{C} & 2.976600 & 1.857785 & -4.455871 \\ \mathrm{C} & 3.110134 & 3.050829 & -3.707124 \\ \mathrm{C} & 3.907332 & 0.840480 & -4.272388 \\ \mathrm{C} & 4.128421 & 3.137108 & -2.762558 \\ \mathrm{C} & 4.968267 & 0.945810 & -3.353250 \\ \mathrm{H} & 3.843784 & -0.052798 & -4.884371 \\ \mathrm{C} & 5.069755 & 2.104877 & -2.566361 \\ \mathrm{H} & 4.247048 & 4.051330 & -2.190286 \\ \mathrm{C} & 5.300601 & 2.317419 & 2.138257 \\ \mathrm{C} & 5.932049 & 1.135220 & 2.596697 \\ \mathrm{C} & 5.384577 & 2.635419 & 0.778087 \\ \mathrm{C} & 6.588037 & 0.340462 & 1.660540 \\ \mathrm{C} & 6.072958 & 1.837103 & -0.156879 \\ \mathrm{H} & 4.951678 & 3.569372 & 0.434410 \\ \mathrm{C} & 6.681980 & 0.655029 & 0.297808 \\ \mathrm{O} & 6.073640 & 0.747293 & 3.896574 \\ \mathrm{O} & 2.253927 & 4.053641 & -4.067449 \\ \mathrm{C} & 1.999584 & 5.161150 & -3.213292 \\ \mathrm{H} & 2.898151 & 5.471855 & -2.666095\end{array}$




\begin{tabular}{|c|c|c|c|}
\hline & 1.754752 & 5.980420 & \\
\hline & 5994369 & -0.181710 & -3.338334 \\
\hline & & & \\
\hline & 4648 & 0.238219 & \\
\hline & 212866 & 2.362409 & \\
\hline & .346027 & 3825 & -1.52 \\
\hline & 7.144986 & 1.986454 & -2.01782 \\
\hline & 7.073637 & -0.5 & 2.045 \\
\hline & 997 & & \\
\hline & 210195 & -0.8 & \\
\hline & 7.924122 & & -1 \\
\hline & -7.321021 & -2.2 & \\
\hline & -4.957486 & & \\
\hline & 042 & & \\
\hline & -5.748303 & & \\
\hline & -9.471430 & & \\
\hline & -10.211083 & 3043 & \\
\hline & 2.793989 & -4.9 & -1 \\
\hline & 065 & -5.9 & \\
\hline & 2.9 & -4 & \\
\hline & 5.03 & & \\
\hline & & & \\
\hline & 5.526694 & & \\
\hline & & & \\
\hline & & & \\
\hline & 69 & & -5 . \\
\hline & 1.8 & & -6 . \\
\hline & 1.6 & -0.2 & \\
\hline & 0.93 & & \\
\hline & 2.65 & & \\
\hline & 6.1 & -4.9 & \\
\hline & 5.9 & -4.2 & \\
\hline & 5.8 & -5.9 & \\
\hline & 7.16 & -4.9 & \\
\hline & & & \\
\hline & & & \\
\hline & 3.5 & & \\
\hline & 820 & 5.0 & \\
\hline & -6.2 & & \\
\hline & -6.9 & & \\
\hline & & & \\
\hline & -1 & -3.7 & \\
\hline & -1.8 & -4.0 & \\
\hline & -1.0 & -4.6 & \\
\hline & -4.7 & 3.3 & \\
\hline & & & \\
\hline & & & \\
\hline & & & \\
\hline & 71 & $-1 . \varsigma$ & \\
\hline & 1.329752 & -3.678961 & 2.6 \\
\hline & 1.311860 & -1.253683 & 4.1 \\
\hline & -0.8 & -1.5 & \\
\hline & & & \\
\hline & & & \\
\hline & 1.33 & -0.0 & \\
\hline & 2.570066 & -1.813542 & 3.71577 \\
\hline & 3.48 & -3.507921 & 2.70 \\
\hline & 2.532845 & 0.696778 & $5.0197 \mathrm{C}$ \\
\hline
\end{tabular}




\begin{tabular}{|c|c|c|c|}
\hline & 80 & 9 & 5.198555 \\
\hline & 3.762610 & -1.097663 & 3964679 \\
\hline & 419 & 524 & \\
\hline & 14860 & 029 & \\
\hline & 708140 & 6340 & \\
\hline & .469355 & 8210 & \\
\hline & -2.624061 & 4.546147 & \\
\hline & 490 & & \\
\hline & 170 & & \\
\hline & .878932 & 326 & \\
\hline & -2.001465 & 2.542131 & -1.6 \\
\hline & -3.7 & 8115 & \\
\hline & & & \\
\hline & 18 & & \\
\hline & 255 & & \\
\hline & & & \\
\hline & -0.7 & 922 & \\
\hline & 0.0 & & \\
\hline & 0.8 & & \\
\hline & 1.2 & & \\
\hline & 961 & & \\
\hline & 987 & -3.1 & \\
\hline & 394 & & \\
\hline & 24 & & \\
\hline & & & \\
\hline & 04 & -5. & -1 \\
\hline & 45 & -2.4 & -3 \\
\hline & & -1.3 & -4 \\
\hline & -0.9 & -5.6 & \\
\hline & & -3 & \\
\hline & & -1 & \\
\hline & & -5 & -1 \\
\hline & 82 & -6 & -0 \\
\hline & & -3.7 & \\
\hline & & & \\
\hline & & -6 & \\
\hline & & -3 & \\
\hline & -4.8 & -2 & -3 \\
\hline & -5.1 & 230 & -4 \\
\hline & -5.3 & -3.8 & -2 \\
\hline & & & -0. \\
\hline & & & \\
\hline & -3.1 & -0 . & 2 \\
\hline & -2.3 & -1. & \\
\hline & -7.8 & -0 & \\
\hline & & & \\
\hline & & & \\
\hline & & & \\
\hline & & & \\
\hline & & & \\
\hline & 2.5 & 346 & 0 \\
\hline & & 391 & 1 \\
\hline & & & \\
\hline & & & 000 \\
\hline & & & \\
\hline & & & \\
\hline & 1.334456 & -0.004607 & -1.9663 \\
\hline & 0.586979 & 1.394842 & 115 \\
\hline
\end{tabular}




$\begin{array}{rrrr}\mathrm{H} & 2.364483 & 1.306686 & -1.328922 \\ \mathrm{C} & 2.783884 & -0.869914 & 0.107578 \\ \mathrm{H} & 2.798091 & -1.482120 & 1.010502 \\ \mathrm{H} & 2.733759 & -1.502060 & -0.778435 \\ \mathrm{H} & 3.666413 & -0.230915 & 0.058551 \\ \mathrm{C} & 0.335346 & -0.871269 & 0.330693 \\ \mathrm{H} & 0.343529 & -1.645142 & -0.438494 \\ \mathrm{H} & 0.371735 & -1.327479 & 1.319429 \\ \mathrm{H} & -0.561888 & -0.260145 & 0.225509 \\ \mathrm{~N} & 1.552279 & 0.001810 & 0.138232 \\ \mathrm{C} & 0.181167 & 3.458798 & 3.411951 \\ \mathrm{C} & -1.260530 & 3.024335 & 3.560780 \\ \mathrm{H} & -1.797481 & 3.108683 & 2.609393 \\ \mathrm{H} & -1.339226 & 1.983680 & 3.893731 \\ \mathrm{H} & -1.727952 & 3.671981 & 4.303105 \\ \mathrm{O} & 0.695262 & 4.337190 & 4.060599\end{array}$

Imaginary Frequency $=0$

$E(R B 3 L Y P)=-4545.97271$ a.u.

\section{M-5aсCh}

Symbolic Z-matrix:

\begin{tabular}{|c|c|c|c|}
\hline & 476 & 14 & 2.743393 \\
\hline & -6.550413 & -1.748385 & 211683 \\
\hline & 360365 & -1.838035 & \\
\hline & 06441 & -2.244373 & \\
\hline & -4.105799 & -2.005821 & \\
\hline & -5.256852 & -2.685162 & \\
\hline & -5.550825 & -1.075760 & 12 \\
\hline & -6.177658 & -0.526475 & \\
\hline & .024104 & -3.375663 & \\
\hline & & & \\
\hline & -7.176336 & 2.26 & \\
\hline & -8.288104 & 1.546889 & \\
\hline & -6.87 & -0.0 & $-1 . c$ \\
\hline & 67 & 44 & \\
\hline & -6.63 & -0.9 & -2.2 \\
\hline & -5.256321 & -1.145484 & -2.456 \\
\hline & -6.678084 & -2.770228 & 2.5 \\
\hline & & & \\
\hline & -7.6 & & -1.5 \\
\hline & -5.1 & -0.1 & 2.3 \\
\hline & -4.2 & -2.9 & 2.8 \\
\hline & -3.6 & -1.3 & 0.3 \\
\hline & -3.4 & $-4.3 c$ & 0.9 \\
\hline & -2.9 & -3 & -0.5 \\
\hline & -7.1 & -0 & \\
\hline & -7.1 & -1.9 & -2.1 \\
\hline & 4.489213 & -2.682073 & -2.6 \\
\hline & 4.673800 & -1.372831 & -3.04316 \\
\hline & 5.37 & -3.20 & -1.6 \\
\hline & & & \\
\hline & & -1.02 & \\
\hline & 6.323 & -2.35 & \\
\hline & 6.47 & $-1.01 \varepsilon$ & -1.48 \\
\hline & 6.984500 & -2.733124 & 30 \\
\hline
\end{tabular}




\begin{tabular}{|c|c|c|c|}
\hline & 55 & 51 & -3.407291 \\
\hline & 3232035 & 4.222626 & -2401278 \\
\hline & 91 & 2.179027 & \\
\hline & 05371 & 3.979046 & \\
\hline & 902617 & 1.966777 & \\
\hline & 3.598788 & 1.487251 & \\
\hline & 5.151137 & 2.861334 & -1.69469 \\
\hline & 4.549751 & 4.711240 & \\
\hline & & 070 & \\
\hline & 228409 & 665 & \\
\hline & 5.660055 & 2.300069 & \\
\hline & 6.802357 & -0.187199 & \\
\hline & 6.267882 & 1.814406 & \\
\hline & 5.24 & 3.30 & \\
\hline & 344 & 0.53 & \\
\hline & 6.415913 & -0.4 & \\
\hline & 2.491974 & 5.374535 & \\
\hline & 2.233479 & 6.137386 & \\
\hline & $3.0 \varepsilon$ & 765 & \\
\hline & 2.13 & 7.16 & \\
\hline & 5.85 & 0.8 & \\
\hline & 5.75 & 0.7 & \\
\hline & 6.88 & 1.17 & \\
\hline & & & \\
\hline & & 3.7 & \\
\hline & 30 & 2.4 & -1 \\
\hline & 7.26 & -1.1 & 1. \\
\hline & & -0.1 & \\
\hline & 8.29 & $-0.7 c$ & -0.3 \\
\hline & & & \\
\hline & -7.2 & -2.3 & 0. \\
\hline & -4.7 & -0.6 & 4.5 \\
\hline & 487 & -3.4 & -3 \\
\hline & -5.6 & 2.4 & \\
\hline & -9.4 & 2.3 & $-0 . C$ \\
\hline & -10.1 & & \\
\hline & 2.6 & -4.2 & -2 \\
\hline & 2.97 & $-5.3 c$ & -2 \\
\hline & 2.8 & -4.027902 & -1 \\
\hline & 5.35 & -0.67 & \\
\hline & & & \\
\hline & & -1.3 & \\
\hline & 5.2 & -4.5 & -1 \\
\hline & 56 & 2.0 & \\
\hline & & 3.5 & -4 \\
\hline & & $2.6 \varepsilon$ & -5. \\
\hline & & & \\
\hline & & & \\
\hline & & & \\
\hline & 12 & -5.0 & \\
\hline & 6.0 & -4.64 & 0. \\
\hline & 575 & -6.155993 & -0.33684 \\
\hline & & -5.0 & -0.75 \\
\hline & & & 3997 \\
\hline & & & $3.31<$ \\
\hline & & 216 & \\
\hline & 5.300361 & 4.116602 & 3.73416 \\
\hline & -6.3265 & 3.222274 & 0.06 \\
\hline
\end{tabular}




\begin{tabular}{|c|c|c|c|}
\hline & 203 & 6981 & \\
\hline & 636410 & 3.759001 & -0.596531 \\
\hline & & & \\
\hline & 69203 & -4.824672 & \\
\hline & .817363 & -4.851190 & \\
\hline & 255 & 8461 & \\
\hline & -4.323514 & 2.569076 & ז7ר \\
\hline & -5.128765 & 4827 & \\
\hline & & & \\
\hline & 872 & & \\
\hline & 496 & 161 & 1. \\
\hline & 269 & -2.3 & \\
\hline & & -2 & \\
\hline & & & \\
\hline & & & \\
\hline & & & \\
\hline & & -2.8 & \\
\hline & & -4 & \\
\hline & 55 & -0.8 & \\
\hline & & & \\
\hline & & & \\
\hline & & & \\
\hline & 2.8 & 519 & \\
\hline & & & \\
\hline & & & \\
\hline & 02 & & 1.4 \\
\hline & 167 & 272 & -0 \\
\hline & & & \\
\hline & -2.8 & & \\
\hline & & & \\
\hline & -3 & 27 & -0 \\
\hline & 44 & & \\
\hline & & & \\
\hline & -1.7 & & -1 \\
\hline & & & \\
\hline & & & \\
\hline & & & -1 \\
\hline & & 5.7 & -0 . \\
\hline & & & \\
\hline & & & \\
\hline & & & \\
\hline & & & -3 \\
\hline & -2.5 & -1 & -3 \\
\hline & & -3 & -3 \\
\hline & -3.6 & -4.2 & -3. \\
\hline & & & \\
\hline & & & \\
\hline & & & \\
\hline & -0. & & \\
\hline & 962 & -0.584487 & -4.09129 \\
\hline & 456 & -5.134718 & -2.56265 \\
\hline & & & -2 \\
\hline & & & \\
\hline & & -4.0 & \\
\hline & & -6. & -5 \\
\hline & 1.417440 & -2.052883 & -3.4865 \\
\hline & -4.9 & -1.970008 & -3.5 \\
\hline & -5.282621 & -1.462222 & $-4.5 c$ \\
\hline
\end{tabular}




$\begin{array}{rrrr}\mathrm{H} & -5.514103 & -2.918714 & -3.514278 \\ \mathrm{O} & -9.080949 & -0.793936 & -0.033917 \\ \mathrm{H} & -8.760994 & -0.804977 & 0.892485 \\ \mathrm{O} & -2.917465 & -1.381970 & 2.896292 \\ \mathrm{H} & -2.183667 & -1.922076 & 2.527232 \\ \mathrm{O} & -7.711153 & -0.979743 & 2.415359 \\ \mathrm{H} & -7.633691 & -0.709346 & 3.346590 \\ \mathrm{C} & -8.369360 & 0.205460 & -0.726738 \\ \mathrm{H} & -8.901840 & 0.364415 & -1.673144 \\ \mathrm{C} & 0.267284 & 0.358419 & 1.820783 \\ \mathrm{H} & 0.032426 & -0.682131 & 2.056664 \\ \mathrm{H} & 0.980682 & 0.709379 & 2.569861 \\ \mathrm{C} & -1.009273 & 1.214830 & 1.853790 \\ \mathrm{H} & -0.782854 & 2.273105 & 1.702954 \\ \mathrm{H} & -1.708503 & 0.912060 & 1.065555 \\ \mathrm{O} & -1.599713 & 1.095059 & 3.132058 \\ \mathrm{H} & -2.143367 & 0.279876 & 3.123937 \\ \mathrm{C} & 1.539293 & 1.670393 & 0.111928 \\ \mathrm{H} & 2.069619 & 1.590268 & -0.838615 \\ \mathrm{H} & 0.715204 & 2.375506 & 0.017078 \\ \mathrm{H} & 2.234293 & 1.997748 & 0.886776 \\ \mathrm{C} & 2.181354 & -0.632571 & 0.623869 \\ \mathrm{H} & 1.803704 & -1.638414 & 0.801183 \\ \mathrm{H} & 2.759361 & -0.600374 & -0.301960 \\ \mathrm{H} & 2.800738 & -0.318576 & 1.464260 \\ \mathrm{C} & 0.107790 & -0.206638 & -0.599379 \\ \mathrm{H} & 0.711952 & -0.405361 & -1.484670 \\ \mathrm{H} & -0.355585 & -1.133197 & -0.254372 \\ \mathrm{H} & -0.652686 & 0.539503 & -0.827940 \\ \mathrm{~N} & 1.012651 & 0.314164 & 0.487669\end{array}$

Imaginary Frequency $=0$

$E(R B 3 L Y P)=-4393.317892$ a.u. 